Florida International University FIU Digital Commons

\title{
Development and evaluation of a catheter deliverable artificial aortic heart valve prosthesis and delivery system
}

Thomas Edward Claiborne III

Florida International University

DOI: $10.25148 /$ etd.FI14060841

Follow this and additional works at: https://digitalcommons.fiu.edu/etd

Part of the Biomedical Engineering and Bioengineering Commons

\section{Recommended Citation}

Claiborne, Thomas Edward III, "Development and evaluation of a catheter deliverable artificial aortic heart valve prosthesis and delivery system" (2008). FIU Electronic Theses and Dissertations. 2371.

https://digitalcommons.fiu.edu/etd/2371 
FLORIDA INTERNATIONAL UNIVERSITY

Miami, Florida

DEVELOPMENT AND EVALUATION OF A CATHETER DELIVERABLE ARTIFICIAL AORTIC HEART VALVE PROSTHESIS AND DELIVERY SYSTEM

A thesis submitted in partial fulfillment of the

requirements for the degree of

MASTER OF SCIENCE

in

BIOMEDICAL ENGINEERING

by

Thomas Edward Claiborne, III 
To: Interim Dean Amir Mirmiran

College of Engineering and Computing

This thesis, written by Thomas Edward Claiborne, III, and entitled Development and Evaluation of a Catheter Deliverable Artificial Aortic Heart Valve Prosthesis and Delivery System, having been approved in respect to style and intellectual content, is referred to you for judgment.

We have read this thesis and recommend that it be approved.

Anthony J. McGoron

Wei-Chiang Lin

Richard T. Schoephoerster, Major Professor

Date of Defense: March 25, 2008

The thesis of Thomas Edward Claiborne, III is approved.

Interim Dean Amir Mirmiran College of Engineering and Computing

Dean George Walker University Graduate School

Florida International University, 2008 
C Copyright 2008 by Thomas Edward Claiborne, III

All rights reserved. 


\section{DEDICATION}

I dedicate this thesis to my wife, Natalie, to my daughter, Madeleine, to our bun in the oven, to my sisters, and to my Dad. This work would not have been possible without their presence, their belief in me, their support, and their love. 


\section{ACKNOWLEDGMENTS}

I wish to thank my committee members for their guidance and support during my work. They have been instrumental in shaping my future. I wish to thank Dr. Richard Schoephoerster for giving me the opportunity to return to the university in order to continue my education. Additionally, I would like to thank him for introducing me to the exceedingly challenging, fun, and interesting world of artificial heart valve prostheses. His guidance, insight, and vision have been inspiring and great motivators in achieving the goals of this work and for illuminating my future goals. I wish to thank Dr. Anthony McGoron for his support during this work. He has generously given his time and effort in the physical absence of our previous Chairperson. I wish to thank Dr. Wei-Chiang Lin for participating on my committee. I wish to thank Dr. Lidia Kos for having faith in me.

I wish to thank my fellow CVEC lab members for their help and support. Siobhain Gallocher was an excellent resource for research advice, technical direction, and camaraderie. Additionally, she developed and provided the valve leaflet material employed in this work. Qiang "Tony" Wang was also very helpful and a good spirited comrade. His medical expertise and lab equipment knowledge provided valuable inputs. Jose Villar helped me get back into the rhythm of academia and to shake the cob webs from my right brain. His positive attitude was motivational.

I wish to thank Leon Gibson of A-1 Precision Machining in Hialeah, Florida. His expertise in design, machining, and fabrication were excellent sources of information and aid to this work. He has enhanced my overall learning experience.

I have greatly enjoyed this work, and I feel that I am doing exactly what I should be doing. I look forward to continuing this work in the future. 


\section{ABSTRACT OF THE THESIS \\ DEVELOPMENT AND EVALUATION OF A CATHETER DELIVERABLE \\ ARTIFICIAL AORTIC HEART VALVE PROSTHESIS AND DELIVERY SYSTEM by}

Thomas Edward Claiborne, III

Florida International University, 2008

Miami, Florida

Professor Richard T. Schoephoerster, Major Professor

Currently, malfunctioning heart valves are replaced via highly invasive and costly open-heart procedures. A new alternative approach is a catheter deliverable or percutaneous heart valve. Current PHV prototypes utilize fixed animal tissue as valves. This research investigated the feasibility of an artificial PHV and the development of a delivery system. A left heart simulator and a tensile tester were used to characterize the hydrodynamics and mechanics of a novel artificial PHV. Test results showed equal or better in vitro hydrodynamic performance when compared to a St. Jude mechanical valve and an Edwards-Sapien PHV, with a mean pressure drop of $<15 \mathrm{mmHg}$ and a mean regurgitation of $<5 \%$. The PHV's exceeded requirements for fixation and radial force. The $24 \mathrm{~F}$ delivery system successfully delivered and deployed a PHV. The work described herein proves the feasibility of an artificial PHV and delivery system and justifies further investigation into its design and function. 
2. SIGNIFICANCE

3. BACKGROUND

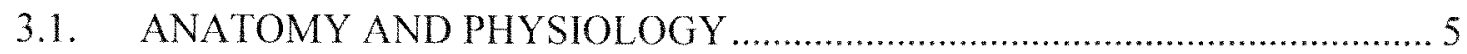

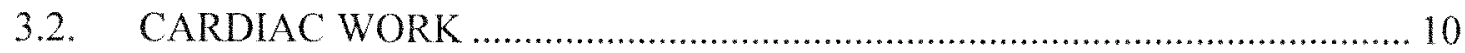

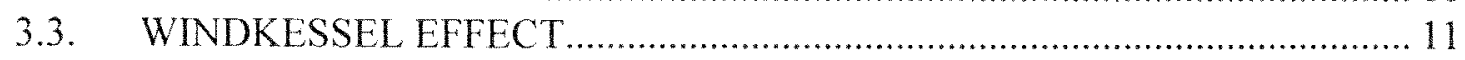

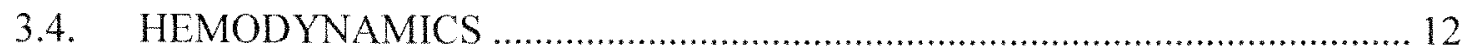

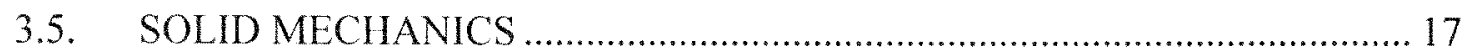

3.6. VALVE DISEASE CLASSIFICATIONS …............................................ 18

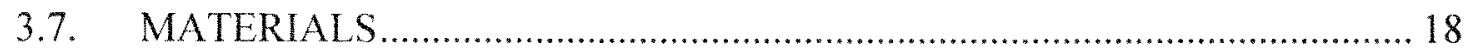

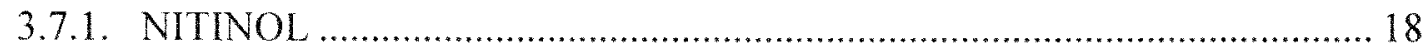

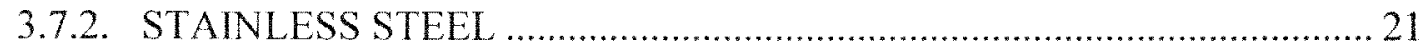

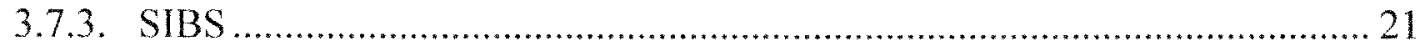

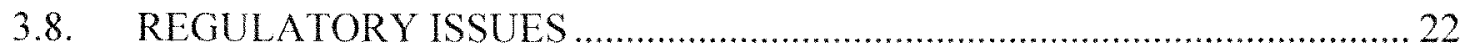

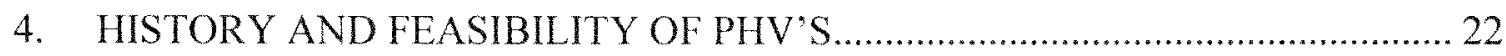

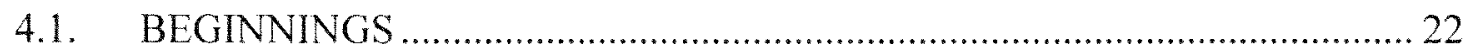

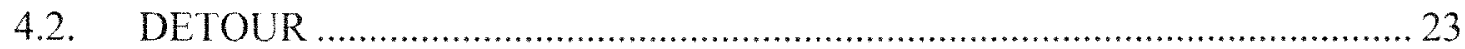

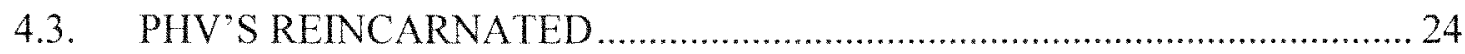

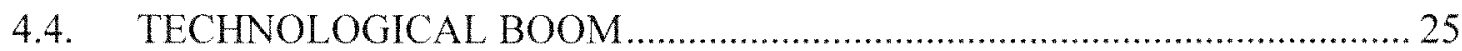

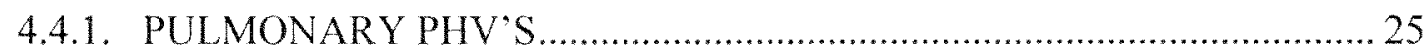

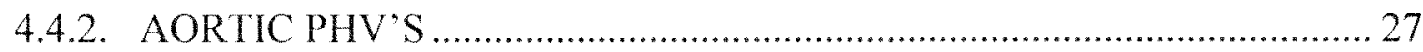

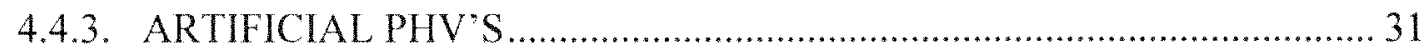

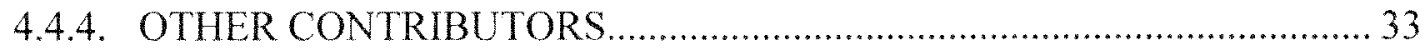

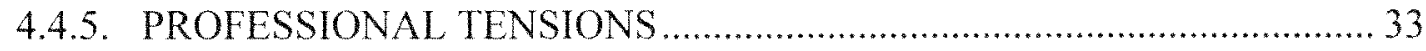

4.5. PHV CLINICAL OUTCOMES AND COMPLICATIONS .............................. 34

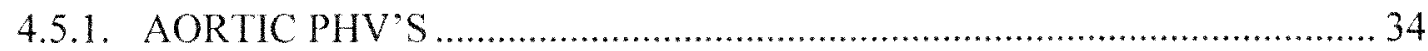

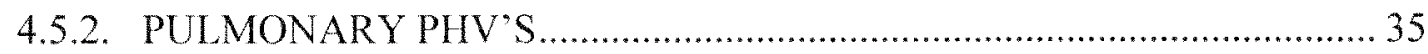

4.6. PHV TECHNOLOGY ANALYSIS AND FUTURE GOALS ….................... 37

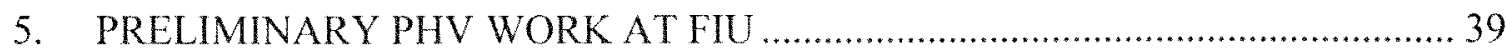

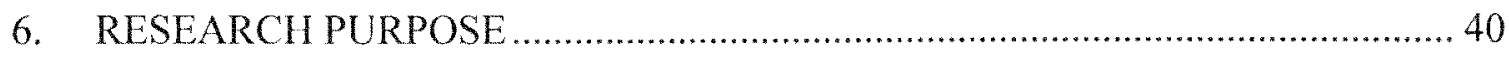

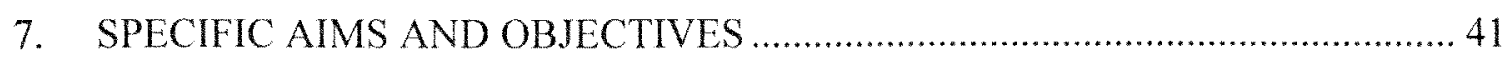

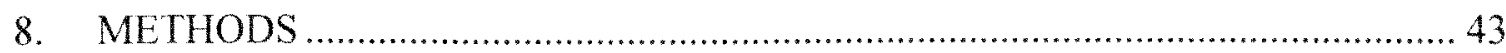




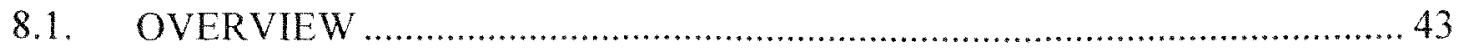

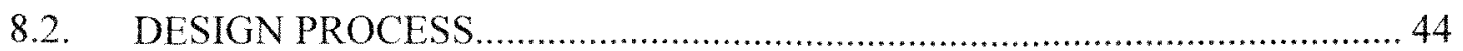

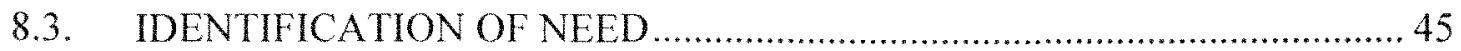

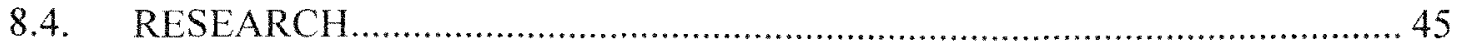

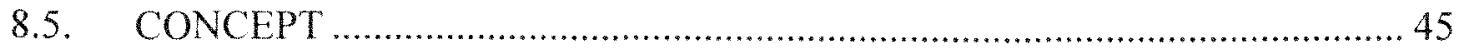

8.6. DESIGN AND FABRICATION …….................................................... 45

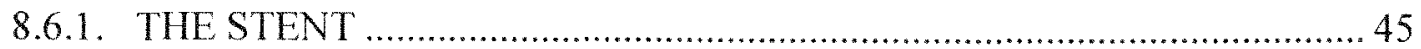

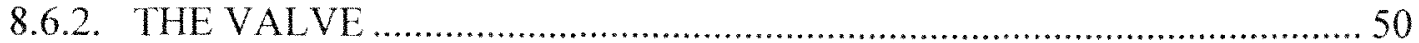

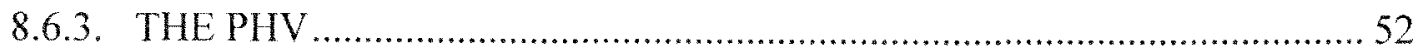

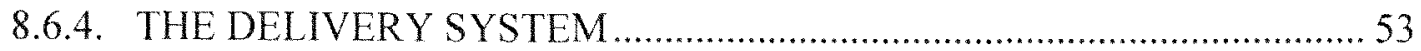

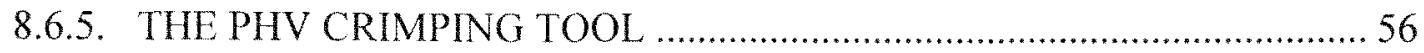

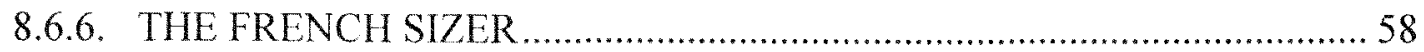

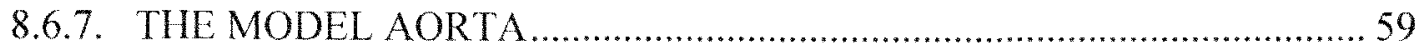

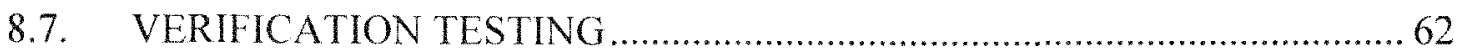

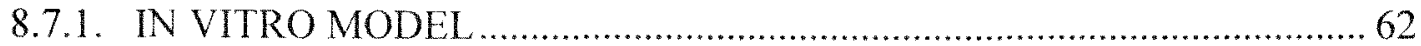

8.7.2. FIXATION

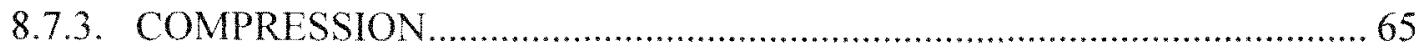

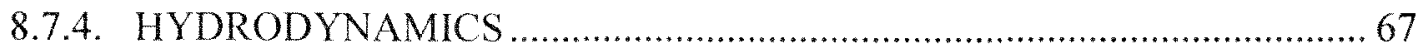

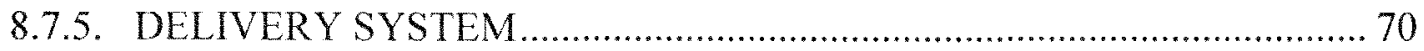

8.8. DATA ANALYSIS AND STATISTICAL METHODS .............................. 72

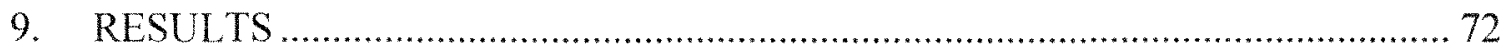

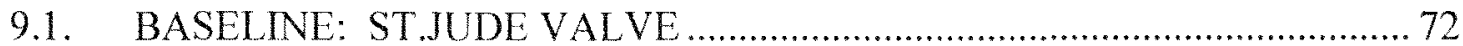

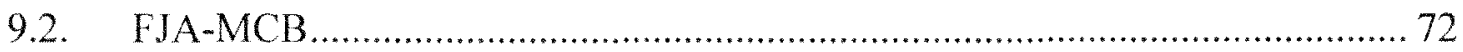

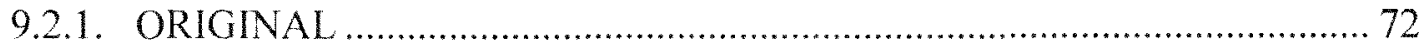

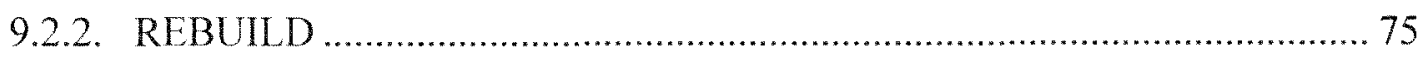

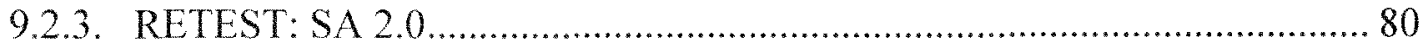

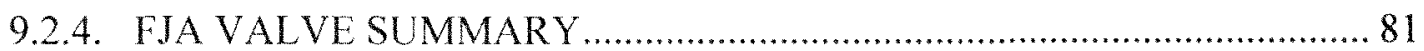

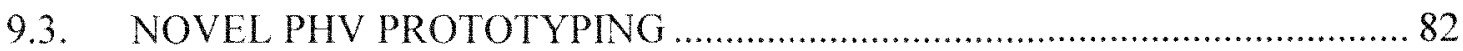

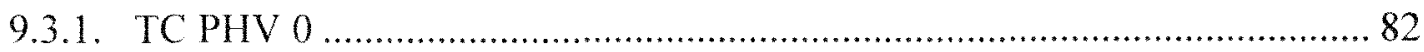

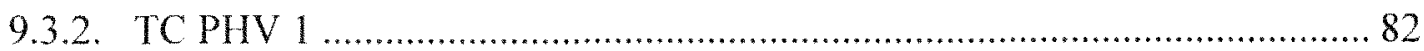

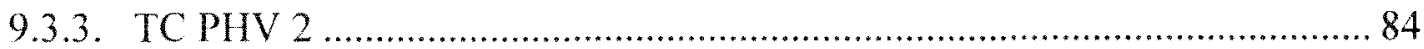

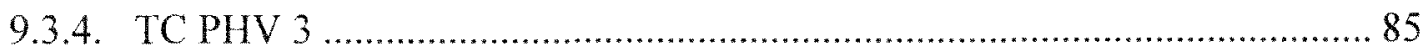

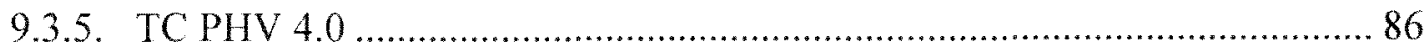

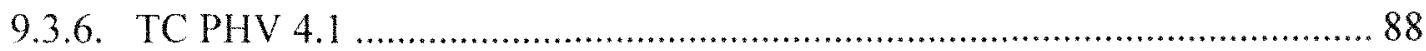

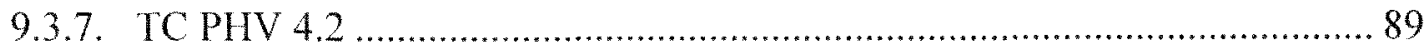

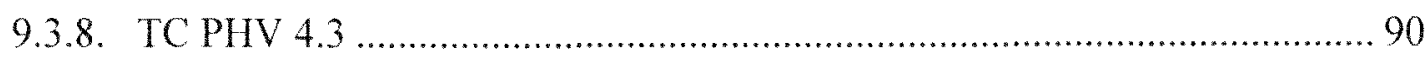

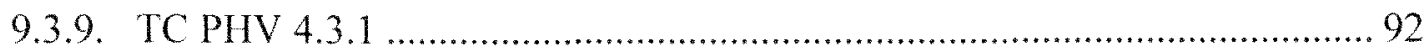

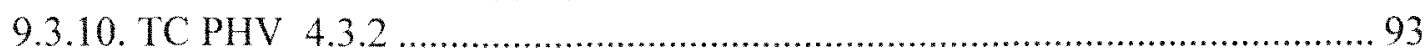

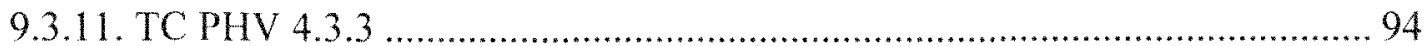

9.3.12. EDWARDS LIFE SCIENCES: SAPIEN (CRIBIER) .............................95

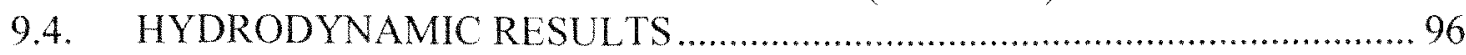

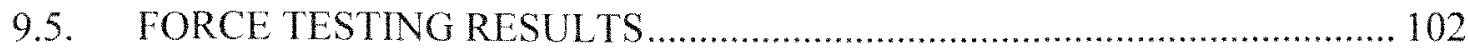




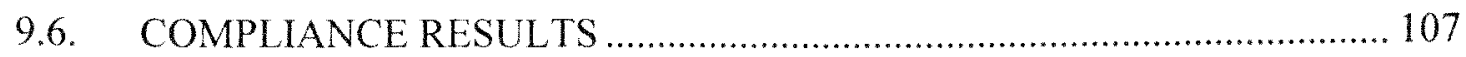

9.7. DELIVERY SYSTEM RESULTS …......................................................... 109

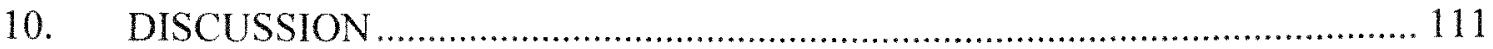

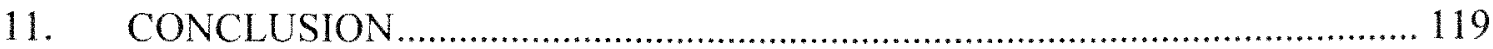

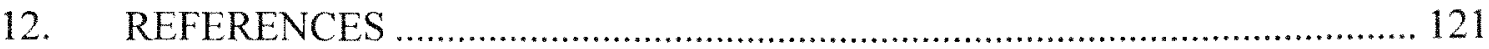

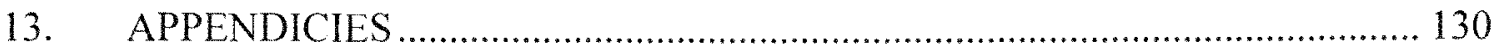


Figure 1: St. Jude Bileaflet Mechanical Valve.

Figure 2: Carpentier-Edwards Perimount Magna pericardial valve. 2

Figure 3: Anatomy and blood flow path in the human heart. 6

Figure 4: Diagram of the aortic valve.. 8

Figure 5: Dissected porcine aortic valve by Claiborne. 9

Figure 6: Electrical analog of a two-element Windkessel model 11

Figure 7: Medtronic Melody TM pulmonary PHV 26

Figure 8: Edwards Life Sciences SapienTM (Cribier-Edwards) aortic PHV.................. 28

Figure 9: CoreValve's Revalving ${ }^{\mathrm{TM}}$ aortic PHV. 29

Figure 10: FDA 21CFR 820.30 Design Control Guidance Diagram............................. 44

Figure 11: Wooden peg board for SS wire forming. 47

Figure 12: Upper SS wire with hypo-tubing. 47

Figure 13: Bench-top Nitinol wire forming ceramic peg board 47

Figure 14: Steel plate for Nitinol wire annealing in a $500^{\circ} \mathrm{C}$ furnace. 48

Figure 15: Nitinol wire formed using the steel annealing plates. 49

Figure 16: Second generation nitinol wire annealing plate. 49

Figure 17: Nitinol wire after annealing in the second generation fixture. 49

Figure 18: Leaflet composite material and drying plate by Gallocher. 50

Figure 19: Dacron-SIBS composite leaflet material 51

Figure 20: PHV shown during assembly. 52

Figure 21: PHV in place on the $19 \mathrm{~mm}$ diameter SA mold spacer...... 53 
Figure 22: Deployment device hand piece

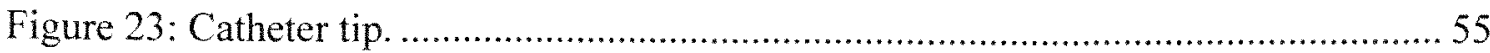

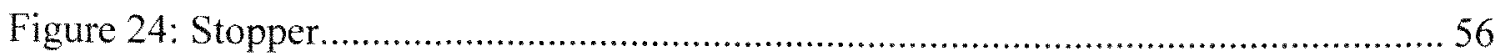

Figure 25: Zeus $24 \mathrm{~F}$ catheter tube with catheter tip and Jagwire. ................................ 56

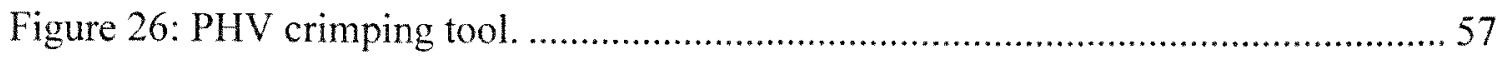

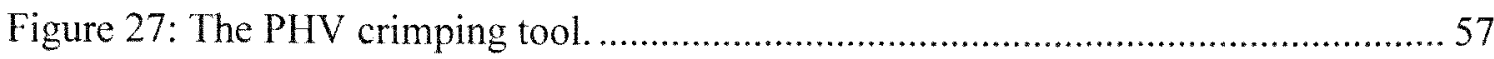

Figure 28: PHV crimping tool shown during the crimping process..............................58

Figure 29: French scale block machined from Delrin. ................................................... 59

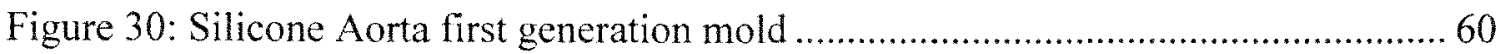

Figure 31: Second generation silicone aorta (SA 2.0) and clam-shell mold. .................61

Figure 32: The silicone aorta clam-shell mold with spacer insert ................................6. 62

Figure 33: A sample of the Matlab Windkessel Model ouput .......................................63

Figure 34: The Matlab Simulink two-element Windkessel model ............................... 63

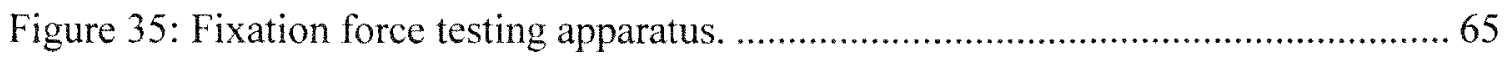

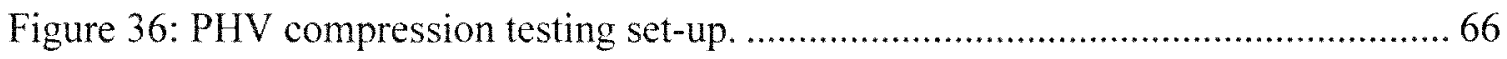

Figure 37: PHV shown inside the compression testing apparatus.................................67

Figure 38: Vivitro Systems, Inc. Superdup'r Left Heart Simulator.................................6 68

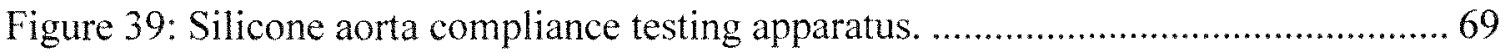

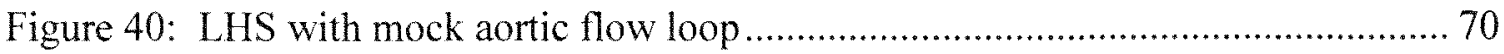

Figure 41: Polycarbonate tube used to test PHV delivery system.................................. 71

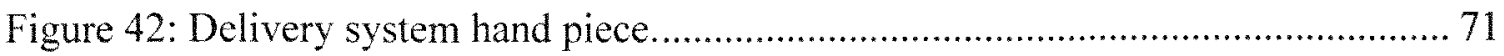

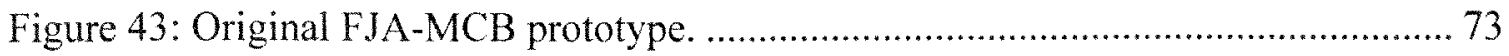

Figure 44: Photo from FJA Thesis showing polymer coated polyester sheet................... 73 
Figure 45: FJA porcine aorta fixture and $\mathrm{MCB}$ valve shown....................................... 74

Figure 46: Porcine aorta inside the LHS during testing........................................... 74

Figure 47: Process of determining wire forming pattern for FJA-MCB. ...................... 76

Figure 48: New FJA-MCB valve by Claiborne …...................................................... 77

Figure 49: SA 1.0 mounted onto the FJA porcine aorta fixture .................................. 78

Figure 50: New FJA-MCB valve inside the first geneneration SA …........................... 78

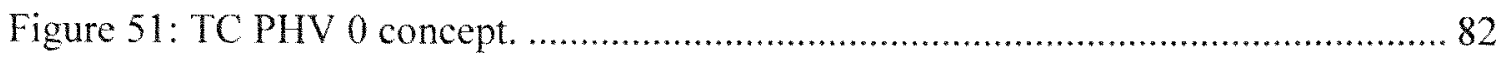

Figure 52: TC PHV 1. The upper bare stent was intended for fixation......................... 83

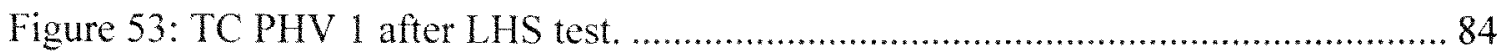

Figure 54: TC PHV 2. Notice the vertical struts and reinforcing sutures....................... 84

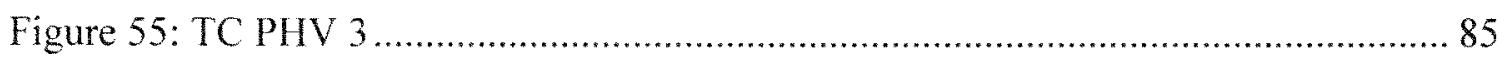

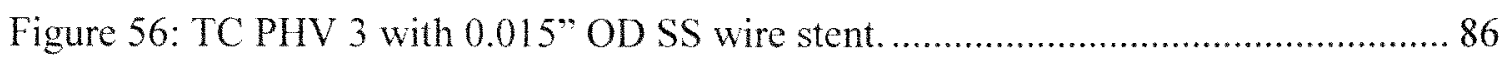

Figure 57: TC PHV 4.0 made with 0.019" OD SEN ....................................................... 87

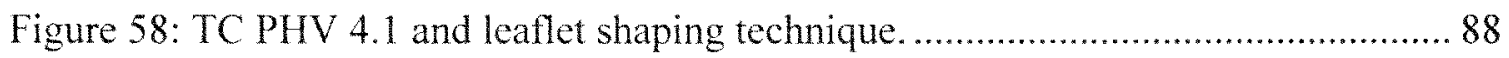

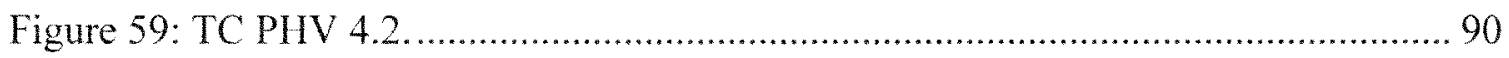

Figure 60: TC PHV 4.3, notice the additional stent in the base of the PHV ................. 91

Figure 61: TC PHV 4.3 inside the $24 \mathrm{~F}$ slot of the French Scale block.......................... 91

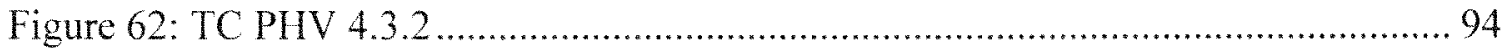

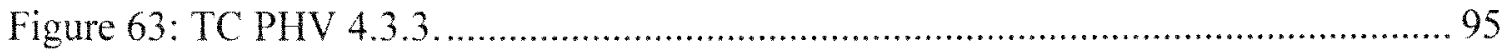

Figure 64: Edwards Life Sciences Sapien valve by Claiborne, ...................................... 96

Figure 65: Photos showing the remote deflection of the catheter tip ........................... 110

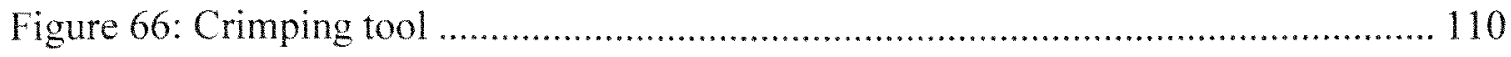

Figure 67: Sequential deployment of the TC PHV 4.3.1 ........................................ 111 


\section{LIST OF CHARTS}

CHART

PAGE

Chart 1: Waveforms generated by the Vivitro LHS 69

Chart 2: New FJA-MCB valve test in first generation silicone aorta 79

Chart 3: New FJA-MCB valve tested in the first generation silicone aorta 80

Chart 4: A comparison of valve performance. 97

Chart 5: A comparison of prototype performance 98

Chart 6: The pressure drop measured across each silicone aorta. 99

Chart 7: ISO 5840:2005 effective orifice area for each prototype. 100

Chart 8: The effect of increasing the SA compliance 101

Chart 9: Results from Bose ELF 3200 radial compression tests. 103

Chart 10: Radial force for each PHV prototype at a diameter of $19 \mathrm{~mm}$ 104

Chart 11: PHV stiffness. 105

Chart 12: The elastic modulus of the Teflon material 106

Chart 13: Results from Bose ELF 3200 axial tensile tests 107

Chart 14: Compliance values measured and calculated on the bench top. 108

Chart 15: Windkessel model results for LHS 109 
Equation 1: $\mathrm{W}=\Delta \mathrm{P} * \mathrm{SV}$

Equation 2: Two-element Windkessel Model: $Q(t)-\frac{p}{R}=C \frac{d p}{d t}$

Equation 3: Systolic Solution: $p(t)=R Q_{0}-\left(R Q_{0}-p_{0}\right) k^{\left(\frac{t}{R c}\right)}$.

Equation 4: Diastolic solution: $p(t)=p_{1} e^{\frac{T-t}{R t}}$

Equation $5: R=\Delta p / Q$

Equation 6: $C=\Delta v / \Delta p$

Equation 7: $C=100 \% \times \frac{d_{2}-d_{1}}{d_{1}\left(p_{2}-p_{1}\right)}$

Equation 8: $\quad \sqrt{\tau}=\sqrt{\tau_{y}}+k_{c} \cdot \sqrt{\gamma}$

Equation 9: $\mathrm{L}=0.06 * \mathrm{D} * \operatorname{Re}$

Equation 10: $\operatorname{Re}=(\rho \mathrm{VD}) / \mu$

Equation 11: $\mathrm{ND}=\operatorname{Re}\left(\mathrm{R}_{\mathrm{V}} / \mathrm{R}_{\mathrm{C}}\right)$

Equation $12: \alpha=(\mathrm{D} / 2)(\omega \rho / \mu)^{1 / 2}$ 14

Equation 13: $\Delta \mathrm{p}=(128 \mu \mathrm{LQ}) /\left(\pi \mathrm{D}^{4}\right)$ 14

Equation 14: $p_{1}-p_{2}=\left\{\left(\rho Q^{2}\right) / 2\right\}^{*}\left\{1 /\left(A_{0}^{2} C_{d}^{2}\right)\right\}$ 15

Equation 15: $\mathrm{C}_{\mathrm{d}}=\left(\mathrm{C}_{\mathrm{C}} \mathrm{C}_{\mathrm{v}}\right) /\left\{1-\mathrm{C}^{2} \mathrm{v}\left(\mathrm{A}_{0} / \mathrm{A}_{1}\right)\right\}^{1 / 2}$ 15

Equation 16: $\mathrm{C}_{\mathrm{C}}=\mathrm{A}_{2} / \mathrm{A}_{0}$ 15

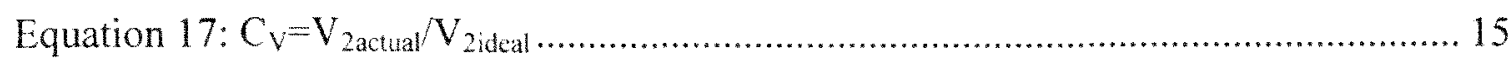

Equation 18: $V_{2 \text { deal }}=\left\{\left[2\left(\mathrm{p}_{1}-\mathrm{p}_{2}\right)\right] /\left[\rho\left(1-\mathrm{C}_{\mathrm{C}}^{2}\left(\mathrm{~A}_{0} / \mathrm{A}_{1}\right)^{2}\right]\right\}\right.$ 15 
Equation 19: $\mathrm{EOA}=\mathrm{A}_{0}=\left(\mathrm{Q}_{\text {mean }} / \mathrm{C}_{\mathrm{d}}\right)\{\rho /(2 \Delta \mathrm{p})\}$

Equation 20: $A_{t O}=\frac{Q_{R A S}}{51.6 \sqrt{\frac{\Delta p}{\rho}}}$.

Equation 21: SV=EDV-ESV 16

Equation 22: $\mathrm{CO}=\mathrm{SV} \times \mathrm{HR}$ 16

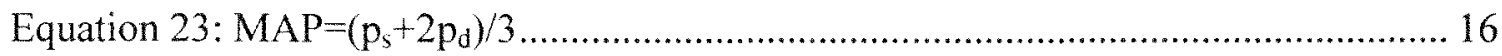

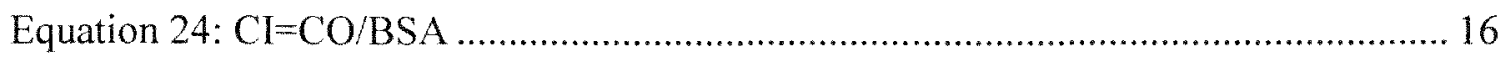

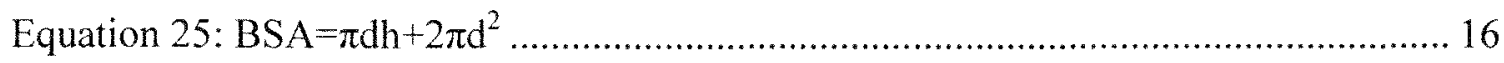

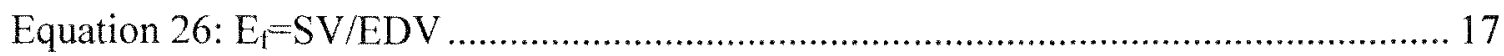

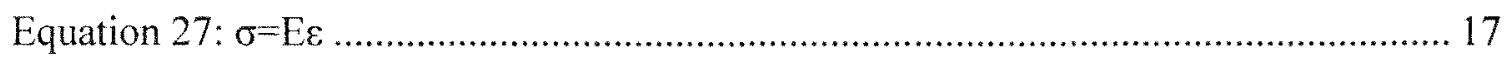

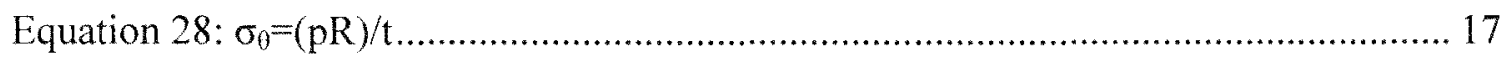

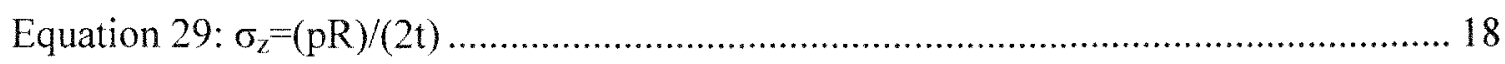

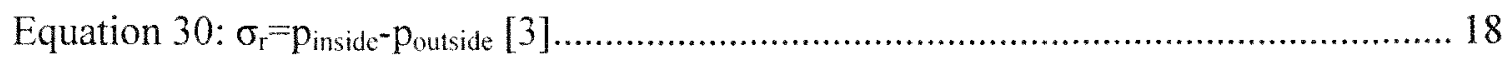

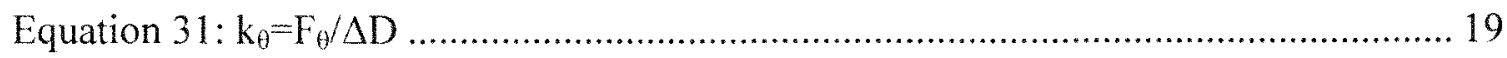

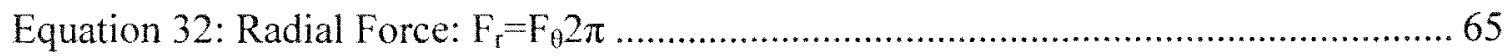




\section{INTRODUCTION}

Commercially available heart valve prosthetics fall into one of two categories; tissue (biologic) and mechanical. The use of mechanical valves began on September 21, 1960 with the first human implantation of the ball-in-cage Starr-Edwards valve. [1] In 1979 a report describing the first human implantation of the St. Jude bileaflet mechanical valve was published by Emery et al. [2] St. Jude's current model is shown in figure 1, and it remains the most popular valve prosthesis on the market today. $[3,4]$

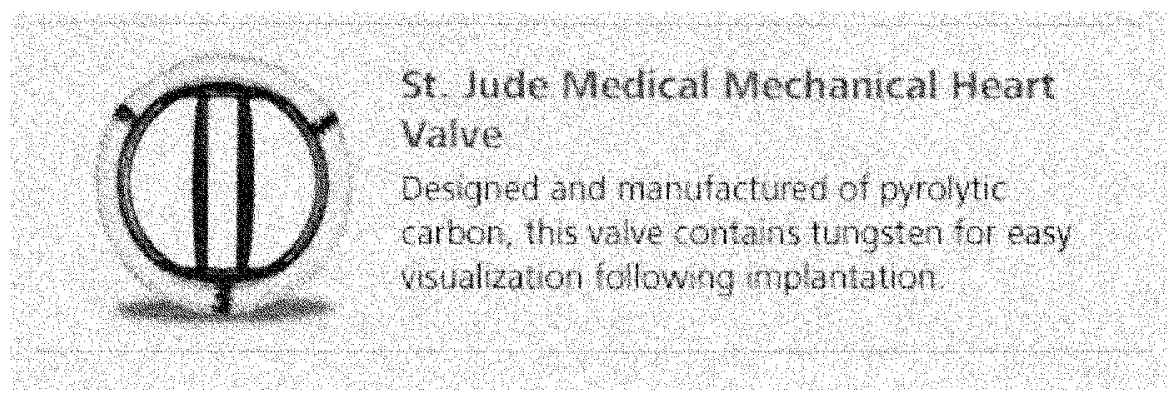

Figure 1: St. Jude Bileaflet Mechanical Valve. Image from http://www.sjm.com/index.aspx.

However, mechanical valves have remained largely unchanged for the past two decades. Mechanical valves are primarily composed of metal alloy frames and pyrolitic carbon leaflets. Approximately $80 \%$ of implanted valves are mechanical bileaflet valves. Mechanical valves are highly durable, with life spans $>15$ years in vivo, but they are thrombogenic and recipients require lifetime anticoagulant drug therapy, hence they are at risk for bleeding disorders. [3] A recent study found the primary failure modes of mechanical valves to be thromboembolism and endocarditis. [5] However, another recent study of "middle aged" patients, of ages 50-65 years, concluded that mechanical valve 
prosthetics remain the best choice for heart valve replacement for that particular population in spite of the associated risks. [6]

In 1970 the Hancock porcine aortic valve was introduced to the market, which is now manufactured by Medtronic. [3] The Carpentier-Edwards pericardial tissue valve followed suit in 1976. [1] Edwards' current model is shown in figure 2.

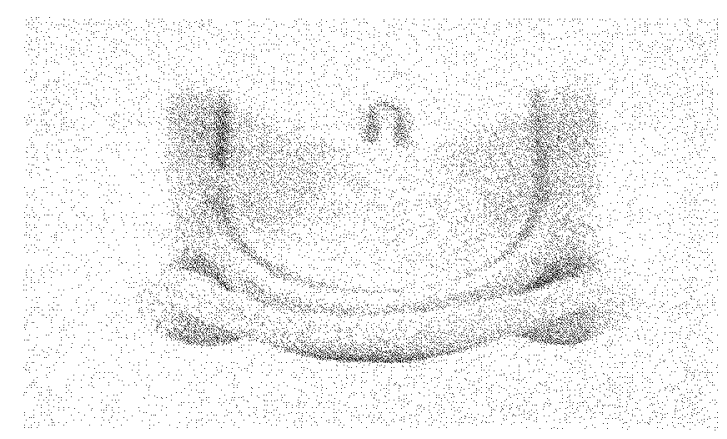

Figure 2: Carpentier-Edwards Perimount Magna pericardial valve. Image from http:/www.edwards.com/products/heartvalves/magna.htm.

Like mechanical valves, only incremental progress has been made since, e.g. the tissue fixation process has improved and extended the average usable life of tissue valves to 10 years or more. $[3,7]$ Tissue valves are typically composed of chemically fixed xenografts, specifically, porcine or bovine tissue fixed with glutaraldehyde. The fixing chemical cross-links the collagen fibers in the tissue providing strength, flexibility, and the prevention of autolysis. Additionally, the chemical treatment reduces antigenicity. The result is non-reactive dead tissue that is flexible and preserved for storage. Patients who receive tissue valves do not require long-term anticoagulant drug therapy, but generally the xenograft tissue begins to degrade via calcification and wear after about 10 years in vivo. [3] Therefore, their use is typically reserved for patients over the age of 65 . 
[6] Furthermore, a recent study found that bovine pericardial tissue valves were about $7 \%$ more durable than porcine tissue valves. [7] However, in general, the in vivo life expectancy of both mechanical and tissue valve prosthetics is dependent upon the individual patient's overall health. $[4,6]$ Moreover, the degradation of tissue valves is age dependent due to the decreasing metabolism of calcium in aging patients. [6]

Overall, the implantation of mechanical and tissue valves has been safe and effective. The mortality rate for all open-heart valve replacement procedures is estimated to be $2-5 \%$. However, the mortality rate for aortic valve surgery is reported to be $17 \%$ and as high as $18 \%$ in octogenarians. [8] Furthermore, open-heart surgery is a highly invasive and costly procedure that requires the use of a cardiopulmonary bypass machine among others. The average post-procedural hospital stay is 1-2 weeks, including 2-3 days in intensive care, and the recovery time varies from a few weeks to several months. As a result patients are at risk for bleeding, stroke, arrhythmias, death, kidney failure, myocardial infarction, medication reactions, breathing difficulty, post-operative confusion, and infection. [9] Moreover, the ideal heart valve prosthesis does not exist. Therefore, shortcomings remain to be solved in improving the design of prosthetic heart valves, e.g. hemocompatibility, durability, implantation method, and hemodynamics. [2, 26] Hence, there exists ample opportunity to find solutions to these issues. Additionally, the trend in cardiovascular interventional medicine in the last decade has been towards minimally invasive procedures. [8] The research proposed here follows that trend logically by building upon the work of other researchers in the development of catheter deliverable heart valve prosthetics, also known as percutaneous heart valves (PHV). In theory, such prosthetics would eventually eliminate most of the risk and cost associated 
with open-heart procedures, and supplant open-heart surgery as the gold standard for heart valve replacement.

\section{SIGNIFICANCE}

Cardiovascular disease is the number one killer of all Americans and is rapidly rising around the globe. The heart is arguably the most amazing organ in the human body. It is a life sustaining electromechanical pump that begins beating during the first trimester of fetal development in the mother's womb and does not stop until death. The heart beats an average of 100,000 times per day. In the average lifetime it will beat more than 2.5 billion times. Failure or impairment of its function can have dire consequences including death. An estimated one in three Americans suffers from cardiovascular disease, and it is the cause of almost $60 \%$ of all American deaths annually. Americans will spend an estimated $\$ 430$ billion in 2007 treating cardiovascular disease. By comparison only $44 \%$ of that amount of money will be spent treating all cancers. [8] Moreover, the world market for heart valve prosthetics is near $\$ 1$ billion. [1]

The aortic valve generally has two modes of failure; stenosis and insufficiency. Stenosis is a reduction in the area of the valve orifice, and insufficiency is a reduction in the valve's ability to stop backward blood flow. A diseased valve usually exhibits some degree of both failure modes. [10] However, aortic valve stenosis is the most common form of valve disease in the developed world, and an estimated $2-4 \%$ of people over the age of 65 are affected. [65] Stenosis is typically caused by a congenital defect, calcification, commissural fusion, or fibrosis; any of which can also cause insufficiency. Insufficiency alone is typically caused by rheumatic heart disease or aortic root dilatation. 
[10] In 2002 the estimated mortality rate for heart valve disease in the U.S. was $20-44 \%$, while the estimated mortality rate for aortic valve disease was $25-54 \%$. The prevalence of left heart valve disease is estimated to be $1-2 \%$ of the American population. Overall, an estimated 99,000 heart valve procedures were performed in the U.S. in 2004, but there are approximately 300,000 performed in the developed world annually. The average cost of a heart replacement procedure was about $\$ 120,000$ in 2005. [8] Therefore, the development of treatment modalities for heart valve disease remains a significant public health issue in the United States of America.

\section{BACKGROUND}

\subsection{ANATOMY AND PHYSIOLOGY}

A thorough understanding of the path of blood flow through the heart is necessary because the four heart valves are the structures that control the direction of blood flow. Figure 3 illustrates heart anatomy.

Oxygen poor blood returning from the venous system (or reservoir, which holds $67 \%$ of blood volume) enters the right atrium of the heart via large low pressure $(<5$ $\mathrm{mmHg}$ ) veins called the inferior and superior vena cava. Once filled, the right atrium weakly contracts creating a pressure gradient across the tricuspid valve separating the right atrium and right ventricle. Then the tricuspid valve opens and blood flows into the right ventricle. The right ventricle is a low-pressure relatively weak thin-walled crescentshaped chamber that functions like a bellows stoking a fire. When the pressure in the right ventricle exceeds the pressure in the right atrium the tricuspid valve closes, and the right ventricle contracts creating a pressure gradient across the pulmonary valve, 


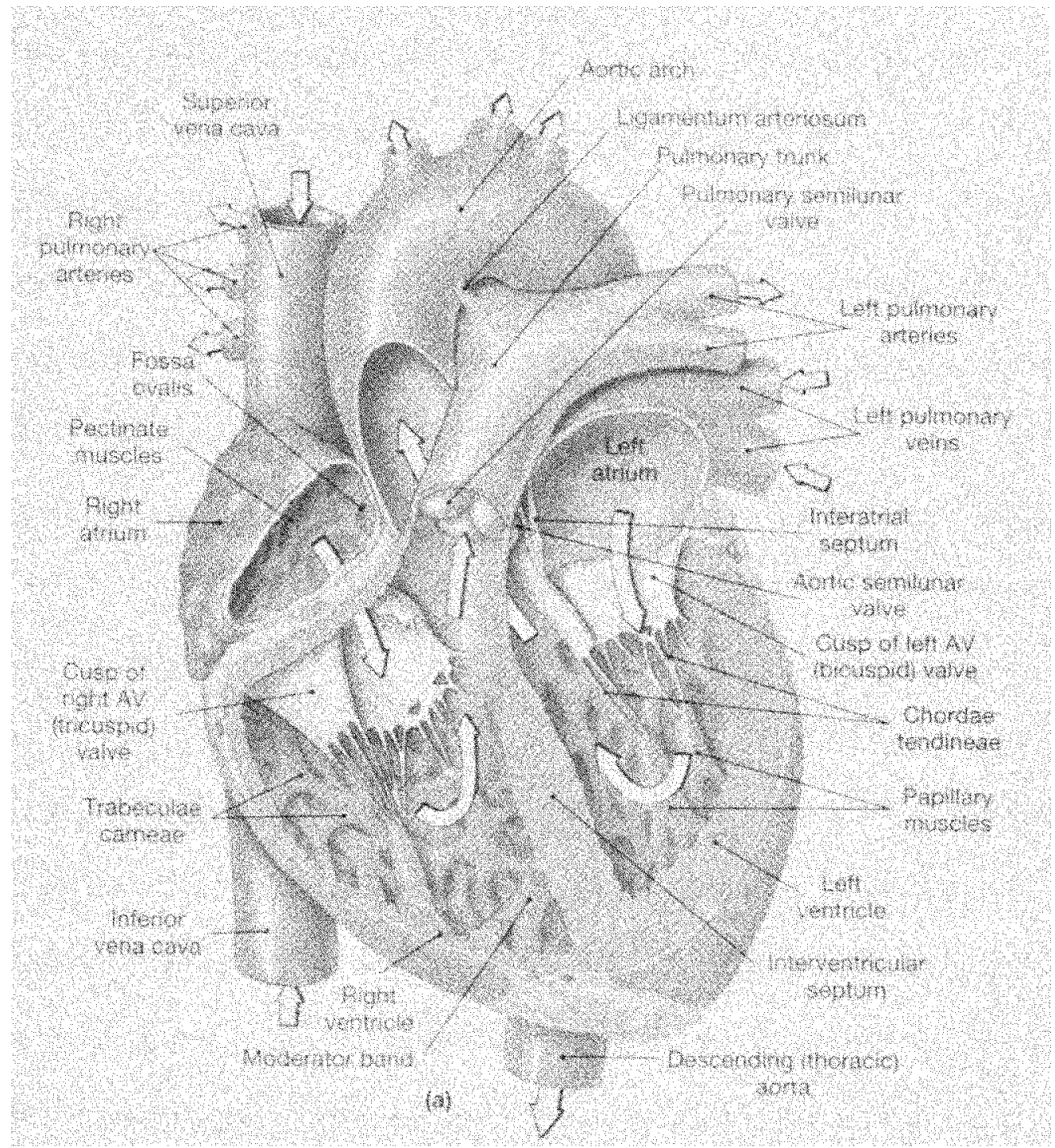

Figure 3: Anatomy and blood flow path in the human heart. Frontal plane cross-section is shown. Image from http:/www.bg.ic.ac.uk/Staff/khparker/homepage/BSc_lectures/2002/Heart_anatomy.jpg

Then the pulmonary valve opens and blood is pumped into the pulmonary artery and on to the lungs. The normal mean pressure in the pulmonary circuit is $10 \mathrm{mmHg}$. The nomenclature of the pulmonary circuit appears opposite to that of all other vascular terminology due to its unique functionality, i.e. the pulmonary artery is the only artery in 
the body which carries oxygen poor blood, and the pulmonary veins are the only veins in the human body which carry oxygen rich blood. The applicable rule is that arteries move blood away from the heart and veins move blood toward the heart. [11]

Likewise, oxygen rich blood returning from the lungs enters the left atrium via two large vessels called the pulmonary veins. The left atrium contracts raising the pressure therein, and when the left atrial pressure is greater than the pressure in the left ventricle, the bicuspid mitral valve opens and blood flows into the left ventricle. The left ventricle is a strong high-pressure thick-walled conical-shaped chamber that functions like a fist wringing a wet towel. When the pressure in the left ventricle becomes greater than the pressure in the left atrium the mitral valve closes and the left ventricle contracts. Then the pressure in the left ventricle rises above the pressure in the aorta, the largest artery in the body, the aortic valve opens and the blood is pumped out of the left ventricle and into the aortic root and on to the organs and tissues. The arterial system has a normal mean pressure of $90 \mathrm{mmHg}$ and contains about $11 \%$ of the total blood volume. Once the pressure in the aorta exceeds the pressure in the left ventricle the aortic valve closes and blood flows into the two coronary arteries located inside the left and right sinuses of Valsalva to feed the myocardium. The sinuses are located just above the aortic valve in the aortic root. The period of atrial contraction and ventricular filling is called diastole, and the period of atrial filling and ventricular contraction is called systole. The normal arterial blood pressure is written as systolic pressure over diastolic pressure and is about $120 / 80 \mathrm{mmHg}$. The period of greatest stress on the aortic valve is when it is closed bearing the load of arterial diastolic pressure. $[3,11]$ 
The special anatomical arrangement of tissues in the aortic valve leaflets is the source of their ability to function for an entire lifetime. Closely modeling the natural leaflet tissue structure may be the key to creating a more durable artificial aortic PHV. The leaflets of the aortic valve are hemispherical in shape and extend from the base of each of three aortic sinuses, as shown in figures 4 and 5 .

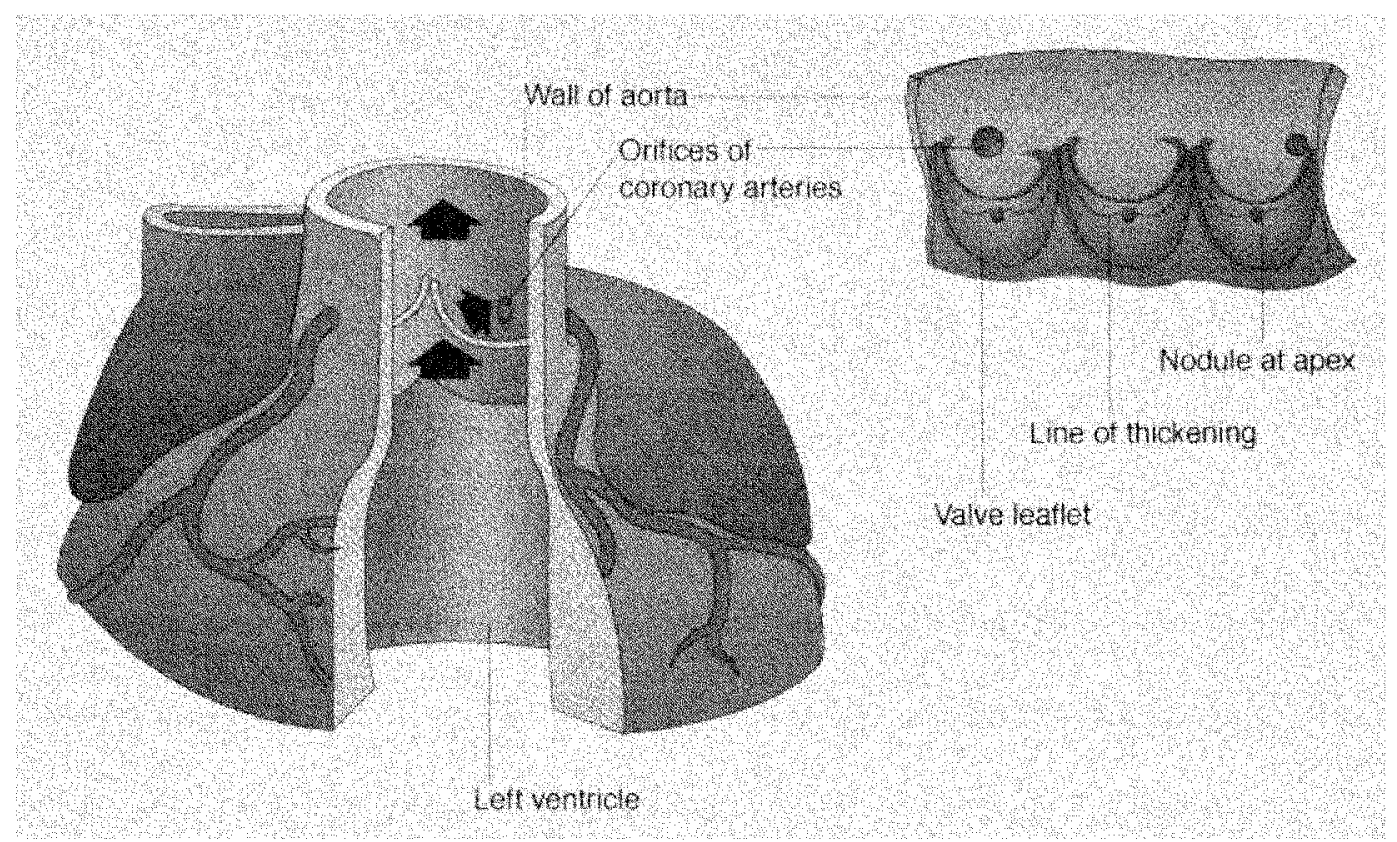

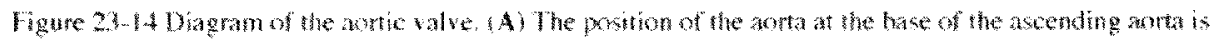

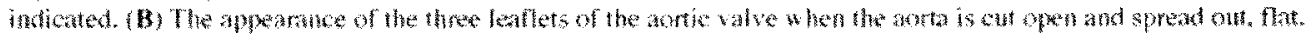

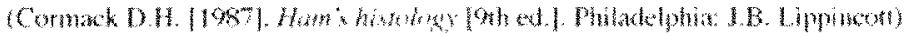

Figure 4: Diagram of the aortic valve. Image from http://connection.lww.com/Products/porth7e/Ch23.asp. 


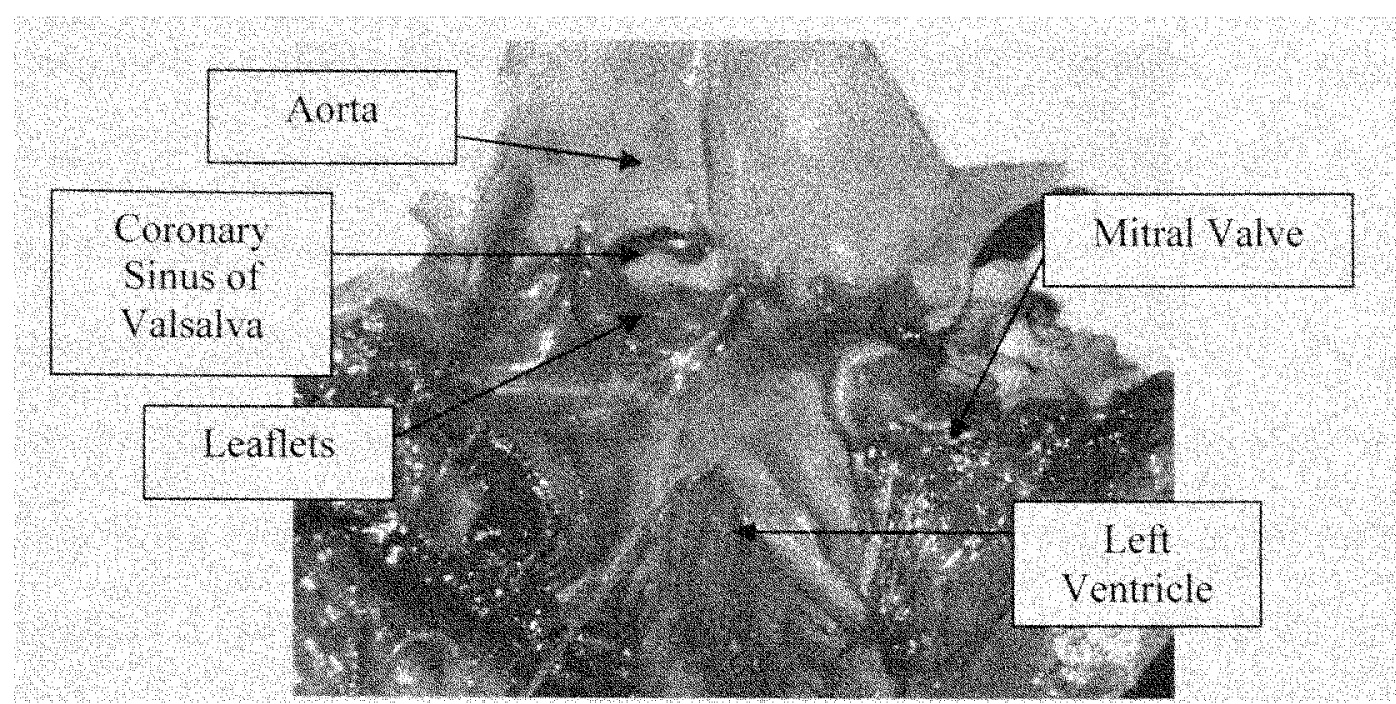

Figure 5: Dissected porcine aortic valve by Claiborne. Frontal plane cross-section shown.

The aortic valve functions as a collapsing cylindrical tube. The leaflets do not simply swing open and closed as a door on a hinge. The leaflets are composed of three tissue layers; the lamina fibrosa, lamina spongiosa, and lamina ventricularis. Additionally, endothelium covers the entire surface of the leaflets. The lamina fibrosa is a dense collagenous tissue on the aortic surface of the leaflets which provides structural strength and integrity. The fibers of the lamina fibrosa are aligned mainly in a circumferential direction which correlates to the stress concentrations in the leaflets during diastole. The surface of the lamina fibrosa is ridged like a gathered sheet of cloth. The lamina spongiosa is the middle layer of the leaflets and is a loose matrix composed mainly of glycosaminoglycans (large proteins that attract water to form a gel) and water. One proposed hypothesis concerning the function of the spongiosa is that it dampens vibrations in the leaflets during the cycle of opening and closing. Another hypothesis suggests that the lamina spongiosa reduces friction in the leaflets by allowing the outermost layers to slide over one another during the opening and closing cycle. Both 
mechanisms would serve to increase the longevity of the valve. In contrast to the lamina fibrosa, the lamina ventricularis is a thinner, weaker, and smooth collagenous tissue on the ventricular surface of the leaflets. The function of the smooth surface is thought to help maintain laminar blood flow during systole. The coaptation surface of the leaflet edges is called the lunula. The lunula is thought to aid in the distribution of the closing forces of the valve as well as forming a tight closure seal. The base of the leaflets is attached to a fibrous band in the aortic root wall called the annulus. The aortic root and annulus are dynamic structures during the cardiac cycle, i.e. they expand and contract as well as move proximally and distally. This movement is impaired by fixed diameter aortic valve prosthetics and may contribute to early prosthetic failure. [10]

\subsection{CARDIAC WORK}

When one or more valves are defective the workload on the heart is increased, and blood flow to and from organs and tissues can be impaired. Cardiac work (W) can be calculated as:

\section{Equation 1: $W=\Delta P * S V$}

In equation $1 \triangle P$ is the pressure difference from systole to diastole and $\mathrm{SV}$ is the stroke volume or volume of blood ejected from the left ventricle during systole. Concomitant acute or chronic negative health changes can occur as a result. For example, aortic valve insufficiency increases SV, which in turn increases the cardiac workload. Over time the left ventricle will dilate due to the volume overload and its walls will become thinner leading to decreased contractility and heart failure. [3] Hence, aortic valve disease is commonly associated with heart failure. [8] 
The aorta's ability to expand and contract like an elastic tube aids blood flow to the coronary arteries and to downstream aortic arterial branch vessels. This elastic recoil also aids in aortic valve closure during diastole. The arteries' capacity to store energy is referred to as the Windkessel effect; this can be modeled as a simple parallel resistorcapacitor circuit as shown in figure 6 and equations $2-4$ :

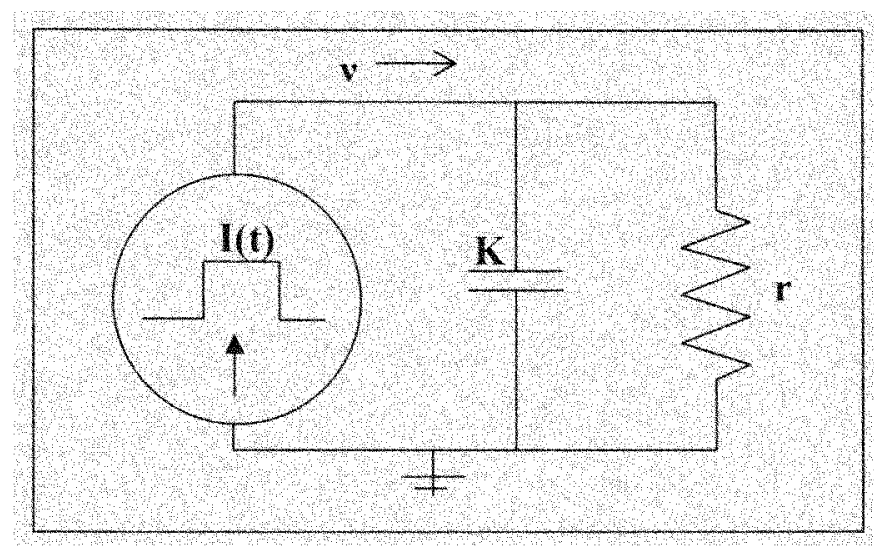

Figure 6: Electrical analog of a two-element Windkessel model, where current (I) is a function of time $(t)$ and is equivalent to flow rate $(Q(t))$, capacitance $(K)$ is equivalent to compliance $(C)$, voltage $(v)$ is equivalent to pressure $(P)$, and electrical resistance $(r)$ is equivalent to vascular resistance $(R)$.

Equation 2: Two-element Windkessel Model: $Q(t)-\frac{p}{R}=C \frac{d p}{d t}$

Equation 3: Systolic Solution: $\quad p(t)=R Q_{0}-\left(R Q_{0}-p_{0}\right) e^{-\left(\frac{t}{k c}\right)}$

Equation 4: Diastolic solution:

$$
p(t)=p_{T} e^{\frac{r-t}{R(}}
$$

In equation $3, P_{0}$ is mean diastolic pressure, $Q_{0}$ is mean flow rate $(\mathrm{ml} / \mathrm{s})$ and is constant, $R$ is resistance (PRU), and $C$ is compliance $\left(\mathrm{ml} / \mathrm{mmHg}\right.$ ). In equation $4 \mathrm{P}_{n}$ is the 
mean diastolic pressure. Blood vessel resistance can be expressed as the ratio of pressure drop to flow rate and is analogous to $\mathrm{Ohm}$ 's Law:

\section{Equation $5: R=\Delta p / Q$}

The units of resistance are peripheral resistance units, where $1 \mathrm{PRU}=1 \mathrm{mmHg} / \mathrm{ml} / \mathrm{s}$. Normal resting vascular resistance is about 1 PRU. Vascular compliance can be expressed as ratio of volume change to pressure change in the blood vessel:

Equation 6: $\mathrm{C}=\Delta v / \Delta \mathrm{p}$

The Windkessel model can be used to calculate resistance and compliance values using $P$ and $Q$ data recorded during in vitro valve testing. [3] However, the equation for compliance $\left(\mathrm{mmHg}^{-1}\right)$ specified in ISO 5840:2005(E) will be utilized in this research:

$$
\text { Equation 7: } C=100 \% \times \frac{d_{2}-d_{1}}{d_{1}\left(p_{2}-p_{1}\right)}
$$

In equation $7, p_{2}$ is systolic pressure and $p_{1}$ is diastolic pressure and $d_{1}$ and $d_{2}$ are the corresponding vessel diameters. A low compliance value would be 0.09 and a high compliance value would be 0.32 . [91]

\subsection{HEMODYNAMICS}

An understanding of fundamental hemodynamics is essential in the design of heart valve prosthetics. Whole blood is considered to be an incompressible Casson fluid, meaning that blood exhibits a small yield stress when a shear stress is applied to a discrete static volume of blood. The viscosity of blood decreases nonlinearly as the applied shear stress increases, i.e. blood is a shear thinning fluid. Therefore, blood tends to coagulate at low shear rates. The constitutive Casson equation is: 
Equation 8: $\quad \sqrt{\tau}=\sqrt{\tau_{y}}+k_{r} \cdot \sqrt{\gamma}$

In equation $7 \tau$ is the shear stress, $\tau_{y}$ is the yield stress, $k_{c}$ is Casson blood viscosity coefficient, and $\mathrm{d} y / \mathrm{dt}$ is the shear rate. This information informs valve designers that low velocity blood flow and recirculation zones near the valve prosthesis should be minimized to prevent thrombosis. [3]

Blood flow in veins and capillaries is considered to be steady, while blood flow in the aorta and arteries is considered to be unsteady. Blood flow in the aorta is not considered to be fully developed because the calculated entrance length (L) is greater than the length of the aorta:

$$
\text { Equation 9: } \mathrm{L}=0.06 * \mathrm{D} * \operatorname{Re}
$$

In equation $8 \mathrm{D}$ is the vessel diameter $(\mathrm{cm})$ and $\mathrm{Re}$ is the Reynolds number:

$$
\text { Equation 10: } \operatorname{Re}=(\rho V D) / \mu
$$

In equation $9, \rho$ is blood density $(1.06 \mathrm{~g} / \mathrm{cc}), \mathrm{V}$ is blood velocity $(\mathrm{cm} / \mathrm{s})$, and $\mu$ is blood viscosity $(3.5 \mathrm{cP})$. The Reynolds number is a non-dimensional ratio of inertial forces to viscous forces. At low Reynolds numbers viscous forces dominate and at high Reynolds numbers inertial forces dominate. Typically, turbulent fluid flow is characterized as having a Re $>4000$. However, in the aortic root and arch the $\operatorname{Re}>5000$, but the flow is mostly laminar due to the curvature of the aorta, the development of Dean Vortices, and the pulsatile nature of the flow. These factors prevent blood cell damage that would be caused by the high shear stresses of fully turbulent flow. The non-dimensional Dean number is:

Equation 11: $N D=\operatorname{Re}\left(R_{v} / R_{C}\right)$, 
In equation $10 R_{V}$ is the vessel radius and $R_{C}$ is the radius of vessel curvature. The magnitude of the Dean number is directly proportional to the magnitude of the effects of vortices or inertia. [3]

Another useful parameter is the Womersley number:

$$
\text { Equation 12: } \alpha=(\mathrm{D} / 2)(\omega \rho / \mu)^{1 / 2}
$$

In equation $11 \mathrm{D}$ is the vessel diameter, $\omega$ is the frequency of fluid oscillation (heart rate), $\rho$ is blood density, and $\mu$ is blood viscosity. The Womersley number is the unsteady version of the Reynolds number and can more accurately describe the flow regime of pulsatile flow. In the aorta $\alpha=18.6$ when $H R=70 \mathrm{bpm}$. Like the Reynolds number, a larger $\alpha$ means that oscillatory inertia forces dominate and a smaller $\alpha$ means that viscous forces dominate. [3]

The classic model of steady Newtonian fluid flow through a uniform rigid tube is called the Hagen-Poiseuille equation:

$$
\text { Equation 13: } \Delta \mathrm{p}=(128 \mu \mathrm{LQ}) /\left(\pi \mathrm{D}^{4}\right)
$$

In equation $12 \mathrm{p}$ is pressure, $\mu$ is blood viscosity, $\mathrm{L}$ is vessel length, $\mathrm{Q}$ is flow rate, and $\mathrm{D}$ is vessel diameter. This equation is derived from the famous Navier-Stokes equations. It is important to note that substituting equation 5 into equation 12 shows that vascular resistance is inversely proportional to the diameter of the blood vessel raised to the fourth power, i.e. changes in blood vessel diameter have a dramatic effect on changes in vascular resistance and blood pressure. This explains why most hypertension treatments include vasodilating drugs. [3] 
When a traditional prosthetic valve is implanted invariably a stenosis is caused in the aortic root. Combining the conservation of energy and conservation of mass equations yields a model for pressure drop across a stenotic valve prosthesis:

$$
\text { Equation 14: } \mathrm{p}_{1}-\mathrm{p}_{2}=\left\{\left(\rho \mathrm{Q}^{2}\right) / 2\right\} *\left\{1 /\left(\mathrm{A}_{0}^{2} \mathrm{C}_{\mathrm{d}}^{2}\right)\right\}
$$

In this model $\mathrm{A}$ is the cross-sectional area at a given position. Furthermore, position 1 is upstream from the valve, position 0 is the surface of the valve, and position 2 is the vena contracta downstream from the valve. The vena contracta is an area of flow issuing forth from the valve orifice wherein the streamlines continue to narrow as fluid flow moves downstream from the valve orifice. The point of minimal cross-sectional area of forward moving flow is called the vena contracta. The discharge coefficient is:

$$
\text { Equation 15: } \mathrm{C}_{\mathrm{d}}=\left(\mathrm{C}_{\mathrm{C}} \mathrm{C}_{\mathrm{V}}\right) /\left\{1-\mathrm{C}^{2} \mathrm{v}\left(\mathrm{A}_{0} / \mathrm{A}_{1}\right)\right\}^{1 / 2}
$$

The contraction coefficient is:

$$
\text { Equation 16: } \mathrm{C}_{\mathrm{C}}=\mathrm{A}_{2} / \mathrm{A}_{0}
$$

The velocity coefficient is:

$$
\text { Equation 17: } \mathrm{C}_{\mathrm{V}}=\mathrm{V}_{\text {2actual }} / \mathrm{V}_{2 \text { ideal }}
$$

The ideal velocity is:

$$
\text { Equation 18: } V_{2 \text { ideal }}=\left\{\left[2\left(p_{1}-p_{2}\right)\right] /\left[\rho\left(1-C^{2} c\left(A_{0} / A_{1}\right)^{2}\right]\right\}\right.
$$

The effective orifice area is given by the Gorlin Equation:

$$
\text { Equation 19: } \mathrm{EOA}=\mathrm{A}_{0}=\left(\mathrm{Q}_{\text {mean }} / \mathrm{C}_{\mathrm{d}}\right)\{\rho /(2 \Delta \mathrm{p})\}
$$

The landmark Gorlin equation published in 1951 is still used clinically today to calculate valve area. $[3,75]$ However, the equation for effective orifice area $\left(\mathrm{cm}^{2}\right)$ specified in ISO $5840: 2005(\mathrm{E})$ will be utilized for this research: 


$$
\text { Equation 20: } A_{E: O}=\frac{Q_{R A S}}{51.6 \sqrt{\frac{\Delta p}{\rho}}}
$$

In equation $19, \mathrm{Q}(\mathrm{ml} / \mathrm{s})$ is the root-mean-square of forward flow, $\Delta \mathrm{p}(\mathrm{mmHg})$ is the pressure drop across the valve during forward flow, $\rho(g / m l)$ is the density of the test fluid, and 51.6 is a constant that allows the units to be correct. [91]

Clinically relevant parameters may also be calculated when designing and testing heart valve prosthetics. Stroke volume is given by the equation:

\section{Equation 21: SV=EDV-ESV}

In equation $19 \mathrm{EDV}$ is the end diastolic volume or the maximum ventricular volume prior to contraction and ESV is the end systolic volume or the amount of blood in the ventricles at the instant of maximal contraction. Normal resting stroke volume is about $90 \mathrm{ml} /$ beat. Cardiac output is the time averaged blood flow rate:

Equation 22: $\mathrm{CO}=\mathrm{SV} \times \mathrm{HR}$

Normal resting cardiac output is $5 \mathrm{~L} / \mathrm{min}$. Mean arterial pressure is defined as:

$$
\text { Equation 23: } \mathrm{MAP}=\left(\mathrm{p}_{\mathrm{s}}+2 \mathrm{p}_{\mathrm{d}}\right) / 3
$$

In equation $21 p_{s}$ is systolic pressure and $p_{d}$ is diastolic pressure. Normal resting mean arterial pressure is about $95 \mathrm{mmHg}$. Cardiac index relates cardiac output to body surface area (BSA):

\section{Equation 24: $\mathrm{CI}=\mathrm{CO} / \mathrm{BSA}$}

The body is considered to be a cylinder of diameter $\mathrm{d}$ and height $\mathrm{h}$ when calculating BSA:

Equation $25: \mathrm{BSA}=\pi \mathrm{dh}+2 \pi \mathrm{d}^{2}$ 
Normal resting cardiac index is about $3.25 \mathrm{~L} / \mathrm{min} / \mathrm{m}^{2}$. Ejection fraction is used to quantify the general health of the heart:

\section{Equation 26: $\mathrm{E}_{\mathrm{f}}=\mathrm{SV} / \mathrm{EDV}$}

Normal resting $E_{f}$ is above $55 \%$. A valve designer can optimize the hemodynamics of his valve prosthesis by employing these analytical equations. [3]

\subsection{SOLID MECHANICS}

A basic understanding of solid mechanics is also useful in valve design. The core relationship often utilized when studying solid material behavior is known as Hooke's Law:

\section{Equation 27: $\sigma=\mathrm{E} \varepsilon$}

In equation $25 \sigma$ is stress (force/area), E is Young's modulus (elastic material constant), and $\varepsilon$ is strain. Strain is a non-dimensional number equal to the change in loaded material length divided by the material's unloaded original length. Generally, stress is plotted as a function of strain and the slope of the line is equal to Young's elastic material modulus. [3]

Large blood vessels, such as the aorta, can be modeled as thin-walled highpressure tubes where the circumferential wall stress, called hoop stress, is calculated as:

\section{Equation 28: $\sigma_{0}=(\mathrm{pR}) / \mathrm{t}$}

In equation $26 \mathrm{p}$ is transmural pressure, i.e. the difference between the pressure inside and outside the vessel, $R$ is the vessel radius, and $t$ is the vessel wall thickness. The subscript $\theta$ denotes the circumferential direction when polar coordinates $(r, \theta, z)$ are specified. Moreover, the axial or longitudinal vessel wall stress is defined as: 
Equation 29: $\sigma_{z}=(\mathrm{pR}) /(2 \mathrm{t})$

The radial stress is simply equal to the transmural pressure gradient:

Equation 30: $\sigma_{\mathrm{r}}=\mathrm{p}_{\text {inside }}-\mathrm{p}_{\text {outside }}[3]$

\subsection{VALVE DISEASE CLASSIFICATIONS}

Clinical valve disease classifications are ubiquitous in literature; therefore a knowledge and context of their meaning is essential to understanding medical reports. Table 1 shows the ACC/AHA valve disease qualitative and quantitative scores. [68]

\begin{tabular}{|c|c|c|c|c|}
\hline Defect & Indicator & Mild & Moderate & Severe \\
\hline \multirow{3}{*}{ Aortic Stenosis } & Jet Velocity $(\mathrm{m} / \mathrm{s})$ & $<3$ & 3 to 4 & $>4$ \\
\hline & Mean Gradient (mmHg) & $<25$ & 25 to 40 & $>40$ \\
\hline & Valve Area $\left(\mathrm{cm}^{2}\right)$ & $>1.5$ & 1.0 to 1.5 & $<1.0$ \\
\hline \multirow{3}{*}{$\begin{array}{l}\text { Aortic Regurgitation } \\
\text { Quantitative }\end{array}$} & Regurgitant Volume (ml/beat) & $<30$ & 30 to 59 & $>=60$ \\
\hline & Regurgitant Fraction (\%) & $<30$ & 30 to 49 & $>=50$ \\
\hline & Reguritant Orifice Area $\left(\mathrm{cm}^{2}\right)$ & $<0.10$ & 0.10 to 0.29 & $>=0.30$ \\
\hline Qualitative & & $1+$ & $2+$ & $3+104+$ \\
\hline
\end{tabular}

Table 1: ACC/AHA Aortic Valve Disease Classifications

\subsection{MATERIALS}

\subsubsection{NITINOL}

Nitinol is an alloy composed of nickel and titanium in a near 50:50 ratio. Superelasticity is defined as full elastic recovery after more than $10 \%$ strain. Nitinol's superelasticity comes from the mechanism by which it elastically deforms. It undergoes a phase transformation when a stress is applied changing from a cubic austenitic crystal structure to a monoclinic martensitic structure. When it is unloaded it changes back to 
cubic austenite. Its mechanical characteristics are highly temperature dependent, but body temperature $\left(37^{\circ} \mathrm{C}\right)$ is within its super-elastic range. $[59,60,62]$

It is biocompatible due to its self-passivating ability to form a stable oxide surface. However, studies have shown that surface finish can have dramatic effects on Nitinol's corrosion resistance and mechanical properties. In fact electropolished Nitinol releases less nickel in vivo, forms a stable titanium-oxide surface layer, and resists corrosion better than mechanically polished or unpolished Nitinol. [60,62]

Furthermore, studies have shown that over-sizing the unloaded diameter of a Nitinol stent by at least $20 \%$ places the radial force and fixation force within the blood vessel at levels required to prevent migration (without an in situ valve). Moreover, the number of wire bends and the diameter of the wire are proportional to the stent's ability to resist collapse, i.e. the radial force exerted by a stent increases with increasing number of wire turns and increasing wire diameter. Furthermore, Nitinol is entirely crush resistant, i.e. it can be crushed flat and it will fully recover to its unloaded shape. According to Duerig et al. 2000 it is preferable to characterize Nitinol stent performance in terms of "hoop stiffness" because it reflects the stent's ability to resist diameter loss:

$$
\text { Equation } 31: \mathrm{k}_{\theta}=\mathrm{F}_{\theta} / \Delta \mathrm{D}
$$

In equation $30 \mathrm{k}_{\theta}$ is the hoop stiffness, $\mathrm{F}_{\theta}$ is the hoop force and $\mathrm{D}$ is the vessel diameter in polar coordinates. Hoop force can be calculated from equation 26 , and $\Delta \mathrm{D}$ can be measured in vitro. It is worth noting that $k_{\theta}$ is equal to the inverse of compliance (equation 5). [59,60]

Heat treating (annealing) Nitinol can alter its mechanical properties by raising its austenitic finish temperature $\left(A_{f}\right)$. This means that at temperatures below $A_{f}$ a Nitinol 
stent can be plastically deformed (called Martensitic or Body Temperature Nitinol), and once it encounters temperatures above its $A_{f}$ it will become super-elastic again. Typically, Nitinol vascular stents are treated so that they have an $A_{f}=28^{\circ} \mathrm{C}$. Additionally, for each degree that $A_{f}$ is below body temperature, the stiffness of Nitinol increases by approximately $4 \mathrm{~N} / \mathrm{mm}^{2}$. [60]

A Nitinol stent can closely match the aortic root compliance, i.e. expanding and contracting, as evidenced by the stress-strain curve for Nitinol, which resembles that of human connective tissue. Furthermore, in vivo it produces a low chronic outward force (COF) that is believed to help maintain a patent vascular lumen. Moreover, it exhibits "biased stiffness" in vivo, i.e. when a crushing force is applied the stiffness of the stent quickly increases from the steady state COF to its original elastic strain plateau. Under cyclic loading Nitinol appears to become stress saturated after about 60 cycles, and then its elastic response becomes constant albeit slightly less than pre-implantation, i.e. the stent stiffens slightly. $[60,62]$

In terms of imaging, it is less non-ferromagnetic than stainless steel and produces low magnetic resonance artifact similar to pure titanium, and it is about equal to stainless steel in X-ray imaging. $[60,62]$

In summary, Nitinol appears to be the best choice of material available for the fabrication of a PHV, but it is expensive, relatively rare, and its properties are highly process and temperature dependent. It requires a skilled metallurgist to perform proper processing in order to achieve the desired properties. Therefore, Nitinol has been reserved for the final PHV design in this research. $[61,87,88,89]$ 
In contrast, stainless steel is narrowly elastic ( $<1 \%$ strain), highly malleable, and about $80 \%$ more rigid than Nitinol. Therefore, it does not require annealing for shape forming, and it cannot match vascular compliance. Its primary advantages are availability, relatively low cost, ease of manipulation, and superior radial stiffness. $[59,60,62]$ However, a stent made from stainless steel will require balloon expansion in situ, i.e. BAV, which associated with complications such as bleeding, arrhythmia, embolism, and other minor complications. [85] Furthermore, initial lab testing has shown that plastically deforming or cold working stainless steel wire embrittles the metal via strain hardening and can lead to fracture. Stainless steel is widely available in a variety of alloys. Generally, higher concentrations of chromium correlate to increased cost, increased corrosion resistance and decreased ductility. $[59,60,62,86]$

\subsubsection{SIBS}

The polymer heart valve promises to be the next generation heart valve prosthesis that should eliminate the need for both tissue and mechanical valves. However, polymer valve development has been beset with complications because a suitable polymer was not available. Ongoing research in the Cardiovascular Engineering Center (CVEC) at FIU has been investigating a novel super-biostable polymer for heart valve prosthetic application called SIBS (poly(styrene-isobutylene-styrene) or SIBS) provided by Innovia, LLC, Miami, Florida. The SIBS thermoplastic elastomer is highly corrosion resistant in contrast to silicone rubbers and polyurethanes previously investigated for heart valve applications. It has excellent biocompatibility and resists degradation in vivo. [94] In the 
CVEC it has been used to construct a novel trileaflet aortic heart valve prosthesis. The valve material developed is comprised of polyester fabric and SIBS. The polyester was employed to reinforce SIBS and prevent creep. [58]

\subsection{REGULATORY ISSUES}

Prosthetic heart valves are classified as Class III medical devices by the United States Food and Drug Administration (FDA) Center for Devices and Radiological Health (CDRH) under the Department of Health and Human Services. In particular, the Code of Federal Regulations (CFR) Title 21 section 870.3925 defines heart valve prosthetics and their requirements for development. Class III devices are defined in 21CFR860.3, and are essentially anything implantable or life sustaining. The design, testing, and performance of heart valve prosthetics are regulated by ISO 5840:2005(E). [91] The FDA recognizes the ISO standard as number 014 in their list Recognized Consensus Standards.

The FDA also has a guidance document entitled "FDA Replacement Heart Valves Draft Guidance, V.5.0". [92] These documents and agencies ensure that minimum performance and safety standards are met when developing prosthetic heart valves.

\section{HISTORY AND FEASIBILITY OF PHV'S}

\subsection{BEGINNINGS}

Percutaneous heart valve (PHV) prosthetics embody the most significant advance in heart valve prosthetic technology in two decades, but it is an idea that has taken some time to germinate. The first report of a minimally invasive treatment for aortic valve insufficiency was published by Hywel Davies in 1965. [12] The valve, manufactured by 
U.S. Catheter Co., Glenn Falls, New York, was essentially a parachute tethered to the end of a $5 \mathrm{~F}$ catheter that was introduced into the descending aorta via peripheral artery access. It was intended to decrease aortic valve insufficiency so that patients could withstand or forgo open-heart valve replacement surgery. Davies reported successful tests in dogs.

Following the work of Davies, in 1971 Moulopoulos et al. reported the creation and testing of similar designs. They fabricated and tested two distinct prototypes. One functioned like an umbrella and the other was a balloon that was inflated and deflated via a machine in synchronization with the cardiac cycle. They tested their prototypes in dogs and reported good results with the umbrella design. [13] Interestingly, their balloonpump composite mechanism was later developed into a widely employed therapy called an intra-aortic balloon pump, which is still used today when acute cardiac support is needed.

In 1976 Phillips et al. followed suit reporting successful animal tests of another similar catheter mounted valve which they described as a polyurethane cusp. However, the use of catheter mounted valves for the treatment of aortic valve insufficiency did not appear to gain wide interest as evidenced by the absence of further reports on the subject. [14]

\subsection{DETOUR}

In 1984 a new minimally invasive valve therapy, percutaneous balloon aortic valvuloplasty (BAV), was presented for the first time by Lababidi et al. for the treatment of aortic valve stenosis. [15] They reported an overall $69 \%$ reduction in the peak systolic 
aortic valve pressure gradient post-BAV. Subsequently, their results prompted wide interest in the technique. BAV gained popularity through the 1980 's, but interest waned through the 1990's because its efficacy was not impressive. Restenosis typically recurred within one year of BAV. Today BAV is used as a palliative therapy for patients awaiting valve replacement surgery or those who are not candidates for said procedure. [65]

\subsection{PHV'S REINCARNATED}

Percutaneous valve implantation regained interest after the publication of the work of Andersen et al. in 1992. [16] They were the first to describe the design of a true untethered PHV. The valve was composed of a porcine aortic valve mounted to two 0.55 mm stainless steel wire stents connected with 2-0 Merseline sutures. The porcine valve was mounted to the stent using 5-0 Prolene sutures. In its collapsed state, the valve was $12 \mathrm{~mm}$ in diameter. The fully expanded diameter of the valve was $32 \mathrm{~mm}$. A $41 \mathrm{~F}$ catheter was used to deliver the PHV via the abdominal aorta of a pig. Their results were not impressive, but they proved the technical feasibility of PHV's. They followed up in 1993 publishing work detailing in vitro and further animal testing of their valve. [71]

Concurrently, in 1992, Pavcnik et al. presented the first artificial PHV concept. [17] The valve was a ball-in-cage design similar to the Starr-Edwards valve; however the cage was made from a self-expanding stainless steel stent and wires, and the base or annulus was a composite of Lycra spandex and stainless steel wires. The cage featured barbs for fixation. The ball was a latex balloon that was filled with a radiopaque "prepolymer" which cured in situ. The assembly was delivered in two stages, each by a $12 \mathrm{~F}$ catheter. The animal results confirmed that the design was an absolute failure, 
which leads one to wonder why in vitro testing of the design did not occur prior to animal testing. Perhaps in deference to their early experience, Pavenik et al. followed up 12 years later by presenting a novel bileaflet catheter deliverable venous valve in 2004. [18]

Moazami et al. followed the work of Andersen and coworkers in 1996 by presenting a bovine pericardial valve mounted to 0.020 inch stainless steel wire stent. [19] Their in vitro results showed regurgitation of $15 \%$ and a transvalvular pressure gradient of $15 \mathrm{mmHg}$ with a mean flow rate of $3.1 \mathrm{~L} / \mathrm{min}$. They implanted their valve into pigs using a $24 \mathrm{~F}$ catheter via direct vision through an aortotomy. This technique required the use of cardiopulmonary bypass. Their in vivo results were ambiguous. As a result, only technical feasibility of their PHV design was proven.

\subsection{TECHNOLOGICAL BOOM}

\subsubsection{PULMONARY PHV'S}

The work by pioneers in the 1990's laid the foundation for a technological boom in beginning of the $21^{\text {st }}$ century. Bonhoeffer et al. catalyzed a rapid precipitation of interest in PHV technology with the 2000 publication of their work describing the first human implantation of a pulmonary PHV. [20] (Earlier that year they had published work describing successful animal trials. [21]) The patient was a 12 year old boy who had a Carpentier-Edwards valved conduit connecting the right ventricle to the pulmonary artery. The conduit exhibited significant stenosis and insufficiency that were causing an enlargement of the right ventricle. Catheterization was performed through the right femoral vein, and the valve was implanted into the conduit via an $18 \mathrm{~F}$ delivery catheter. They reported an uneventful case and a successful outcome. Their valve design was 
composed of an $18 \mathrm{~mm}$ bovine jugular vein segment containing an in situ venous valve mounted inside a balloon expandable platinum-iridium alloy stent. Subsequently, their pulmonary PHV design, now called Melody shown in figure 7 , is in clinical trials with Medtronic.

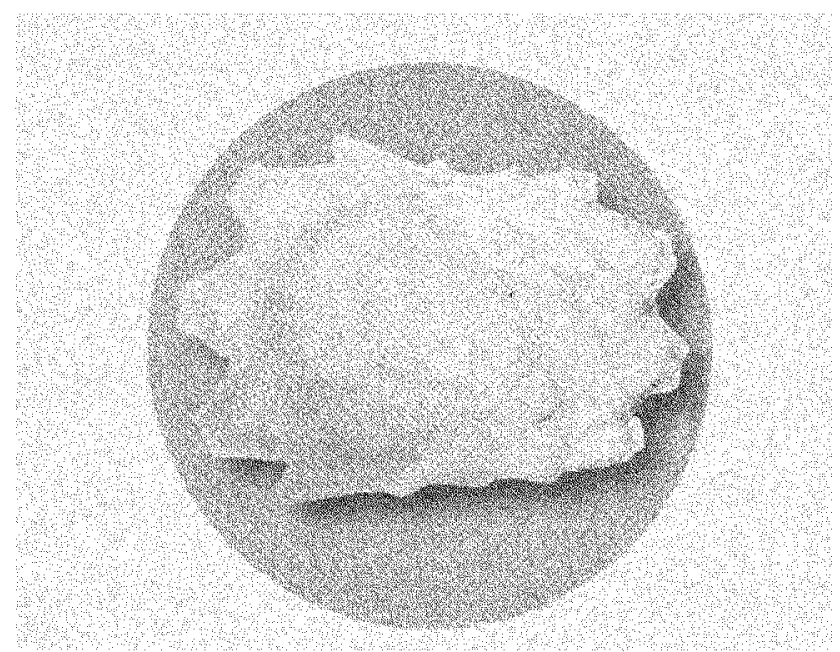

Figure 7: Medtronic Melody TM pulmonary PHV. Image from http://www.medtronic.com/intl/melody/.

Lutter et al. presented two studies detailing the design and animal testing of a pulmonary PHV similar to the Bonhoeffer valve. $[32,35]$ Lutter and coworkers attached a bovine jugular vein segment with an in situ venous valve to a self-expanding Nitinol stent. They delivered the stent into the pulmonary valve position via a $24 \mathrm{~F}$ catheter with some success and proved the feasibility of their design. They noted that the radial force of their stent was $0.1 \mathrm{~N}$ at a diameter of $20 \mathrm{~mm}$ and $37 \circ \mathrm{C}$. Additionally, they noted that $20 \%$ oversizing of the stent diameter provided sufficient anchoring. They sited stent migration in a $12 \%$ oversized stent as evidence. 
Feinstein et al. published a human case report in 2006 wherein they built a pulmonary PHV and implanted it into a 10-month old female. [46] Their work is the first reported on a patient so young. The valve was composed of a Palmaz stent and a porcine valve taken from a Hancock valved conduit. The valve was sutured to the stent using 6-0 and 7-0 Goretex and Prolene sutures. It was delivered via a $10 \mathrm{~mm}$ catheter through the jugular vein. The procedure was a success, and one year out the patient was doing well.

\subsubsection{AORTIC PHV'S}

Another landmark event occurred in 2002 when Cribier et al. reported the first human case of aortic PHV implantation using an antegrade approach. [24] Their valve was composed of bovine pericardial leaflets mounted inside a balloon expandable stainless steel stent. The valve was delivered via a $24 \mathrm{~F}$ catheter inserted in to the femoral vein. The atrial septum was punctured and dilated with a $10 \mathrm{~mm}$ balloon. The catheter was advanced across the mitral valve and into the aortic valve. They reported a $70 \%$ reduction in the transvalvular pressure gradient. Their design had been developed and vetted by Percutaneous Valve Technologies, Inc. of Fort Lee, New Jersey. Currently, their valve, now called Sapien (Cribier-Edwards) shown in figure 8, is in clinical trials with Edwards Life Sciences. (Subsequently, the first human case of pulmonary valve replacement using the Sapien valve was reported by Webb et al. in 2006. [27])

Concurrently, Bonhoeffer and Boudjemline published work in 2002 describing an altered version of their pulmonary PHV design for use in the aortic valve position. [72] They reduced the area of tissue coverage on their original PHV so as not to block the 
coronary ostea, and they added an outer Nitinol stent that expanded into the sinuses of Valsalva for fixation.

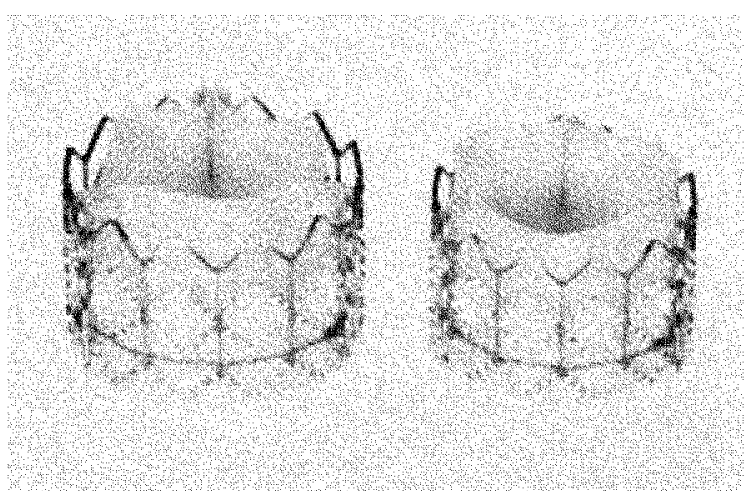

Figure 8: Edwards Life Sciences SapienTM (Cribier-Edwards) aortic PHV. Image from http://www.edwards.com/products/percutaneousvalves/sapienthv.htm.

As a result, the delivery process was carried out in 4 stages: delivery, deployment of the Nitinol stent, balloon expansion of the PHV, and removal of the balloon and catheter. Unfortunately, the results indicated a failure of their design as evidenced by complications such as coronary obstruction, mitral valve interference, and PHV insufficiency. Additionally, they experimented with implanting their original pulmonary PHV design into the descending aorta. [73] In that publication they reported marginal success.

In 2004 Ferrari et al. presented a novel aortic PHV which was tested in pigs. [28] Their design was composed of a porcine pulmonary valve mounted inside a Nitinol stent. The stent featured barbs for fixation and the delivery catheter was 25 French. They reported favorable results, including no observed damage to the aortic intima caused by the stent barbs. However, the in vivo test only lasted six hours and they did not perform a microscopic examination of the intimal tissue. 
In 2005 Grube et al. reported the first human implantation of CoreValve's first generation self-expanding aortic PHV shown in figure 9. [29]

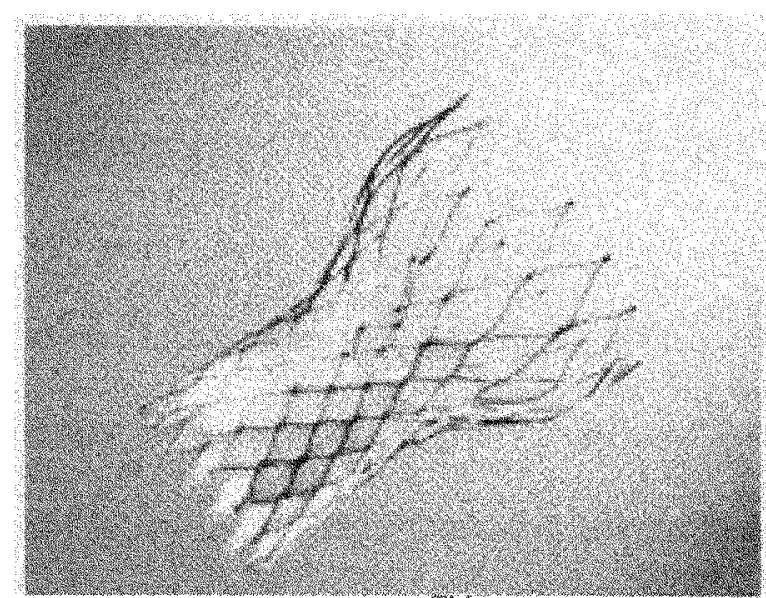

Figure 9: CoreValve's Revalving ${ }^{\mathrm{TM}}$ aortic PHV. Image from http://www.corevalve.com/products.aspx\#.

The tested valve design was composed of a bovine pericardial valve mounted inside a self-expanding Nitinol stent that was laser cut from a $50 \mathrm{~mm}$ long Nitinol tube. The iliac artery was exposed via surgical cut-down, and the femoral artery and vein were cannulated for extracorporeal circulatory support. They reported a $79 \%$ reduction in the transvalvular pressure gradient, and a $40 \%$ increase in the ejection fraction. No regurgitation was found.

Laborde et al. reported the design and animal testing of a variation on the CoreValve PHV in 2005. [43] The valve was composed of the bovine pericardial tissue valve used in the original CoreValve and an assembly of Z-stents made from Conichrome wire, which they claimed had excellent strength. They used 4-0 and 5-0 Polypropylene suture to hold the entire assembly together. The PHV could be crimped to $25 \mathrm{~F}$ and was self-expanding. They implanted the valve into 14 sheep. The design saw the addition of 
hooks to the proximal end of the stent after it sprang into the left ventricle upon deployment. Their results proved the design to be a failure, e.g. the valves migrated postdeployment and showed significant insufficiency. Interestingly, in 2006 they reported a human case of original CoreValve implantation. [44] It seems that Dr. Laborde had entered into a business arrangement with CoreValve at sometime before the publication of their 2006 paper.

Lutter and coworkers published work in 2005 describing the design and testing of an aortic PHV. [30] It was composed of a self-expanding Nitinol stent with barbs for fixation and either a porcine aortic valve or pericardial valve. They reported in vitro test results of 0 to +1 regurgitation and less than $7 \mathrm{mmHg}$ transvalvular pressure drop. In vivo animal test results were marginally successful with similar hemodynamic value reported.

The first human case of retrograde implantation of an aortic PHV was claimed by Paniagua et al. in 2005. [25] Here, PHV means "Paniagua Heart Valve." The entry point was the femoral artery accessed via surgical cut-down, and the delivery catheter was 16 F. They began developing a novel aortic PHV in 1998. They did not provide a detailed description of the valve, but it appeared to have been an animal valve mounted inside a metal stent. Strangely, from this report it appears that they have focused entirely on the valve itself rather than a valve-stent composite device because of a statement that reads, "To deliver the device, we used a Cheatham-Platinum...stent..." Simply testing an explanted animal valve does not constitute a PHV. Their results seem dubious as well. They reported zero transvalvular gradient and regurgitation in this case, as well as in animal and in vitro trials. The patient died 5 days post-implantation of undetermined 
causes. Adding to the incredulity, Paniagua erroneously claimed to have been the first to perform animal testing of a PHV in June 2000, when Andersen and coworkers were clearly first in 1992. However, his claim in the 2005 paper seems to be legitimate.

In a dubious 2007 study, Fletcher and coworkers reported the in vitro study of a PHV. [45] It appears that they did not intend to design a novel PHV, but rather they intended to show how a PHV could obstruct the coronary ostea. So they designed and built a PHV that did exactly that. It was constructed from a Medtronic cobalt-nickel stent and ovine pericardium. Explanted pig hearts served as their in vitro model.

\subsubsection{ARTIFICIAL PHV'S}

In terms of artificial PHV's, Sochman and coworkers have been the pioneers of late. They followed the work of Pavcnik et al. and in 2000 reported successful animal trials of an artificial aortic PHV. [22] Their valve was composed of a polyurethane coated stainless steel disc mounted inside a stainless steel Gianturco Z-stent. They made one PHV "cage" with barbs for fixation and one without barbs. The stent and disc were delivered separately each by a $10 \mathrm{~F}$ catheter. Teflon suture (5-0) was used to engage and lock the disc in place. The PHV was implanted into four dogs. The non-barbed cage did not remain fixed and migrated into the ascending aorta. The barbed cage remained where deployed but was interfering with the coronary ostea. They reported easy deployment of the disc in all locations. Additionally, they reported favorable disc movement, no transvalvular pressure gradient, and no macroscopic intimal damage. They followed up in 2006 with a revised polymer disc PHV design that failed outright in animal tests. [23] 
Webb and coworkers reported the development and testing of a novel PHV design in 2004 for use in the pulmonary or aortic position. [27] Their design was unique in that it was a coiled Nitinol sheet which contained a bovine pericardial valve. The delivery catheter was $22 \mathrm{~F}$. When the valve was deployed into the vessel it automatically uncoiled and retained its maximum expanded diameter via a locking ratchet built into the stent. However, the final expansion and locking of the stent ratchet required balloon dilatation in vivo. Curiously, they reported the performance of in vitro and animal tests but did not include any data. Instead they reported a qualitative analysis coupled with a list of design requirements.

In 2005 Levi et al, reported the design and testing of a bicuspid pulmonary PHV intended for use in smaller pediatric patients. [42] They called their design a "covered stent heart valve" or CSV. The CSV was composed of a modified Palmaz P308 stent mounted inside a Goretex vascular graft conduit. Sections of the stent were removed and the graft was cut such that two "leaflets" could collapse inside the lumen of the stent. A second Goretex conduit was placed over the assembly and was bound to the stent by $6-0$ Prolene sutures. Several variations were built and tested. The delivery catheter ranged in size from $7-10 \mathrm{~F}$. In vitro results showed up to $41 \%$ regurgitation and transvalvular pressure gradients over $300 \mathrm{mmHg}$ at less than $1 \mathrm{~L} / \mathrm{min}$ of flow through a $19 \mathrm{~mm}$ diameter PVC tube with a $16 \mathrm{~mm}$ diameter stenosis region for valve placement. In spite of the poor in vitro results, the prototypes were tested in vivo by implanting them into the descending aorta of pigs, but only qualitative results were reported.

In 2006 Lutter et al. presented a novel artificial pulmonary PHV design composed of a polyurethane trileaflet valve mounted inside a Nitinol stent. [36] No test data 
concerning their design has yet been published. However, polyurethane is well known to be an inadequate material for heart valves.

\subsubsection{OTHER CONTRIBUTORS}

Lutter and team have been prolific in studying PHV technology and participating clinical trials with Sapien, Melody and CoreValve. $[30,31,32,33,34,35,36,37,38,39,56]$ They have also been the first to publish work describing the first human cases of endovascular laser and water aortic valve ablation. They hypothesize that removal of the diseased aortic valve prior to PHV implantation could improve PHV safety and efficacy. Additionally, Zajarias et al. reported successful coronary catheterization after implantation of the Sapien PHV. [74]

In summary, there have been many contributors to modern PHV technology, but Bonhoeffer and Cribier (and coworkers) can safely be called the fathers of modern PHV technology. Moreover, several private commercial entities are currently developing PHV's, but gaining access to their information is not possible due to proprietary constraints.

\subsubsection{PROFESSIONAL TENSIONS}

The speed of progress in aortic PHV technology during this decade has caused controversy between the rank and file of cardio-thoracic surgeons and interventional cardiologists. A 2005 report published by a group of physicians composed of cardiothoracic surgeons and interventional cardiologists (notably lacking any PHV inventors) was intended to embody an effort to establish cooperation, quell controversy, and create clinical standards for the future development of PHV's. [49] First, the report established 
open-heart valve replacement as the "gold standard". Then it claimed that no PHV trials to date had been randomized or controlled, and as a result data had been biased and in some cases incomplete. Additionally, it mandated cooperation and collaboration between cardiac surgeons and cardiologists. Finally, it outlined several recommendations concerning the performance of clinical trials of aortic PHV's.

In summary, the report recommended that aortic PHV study candidates should be symptomatic inoperable patients whose long-term survival is compromised. It speculated that such a selection of patients would illuminate short and mid-term durability and the safety and efficacy of aortic PHV's. The effectiveness of their effort has yet to be proven.

\subsection{PHV CLINICAL OUTCOMES AND COMPLICATIONS}

\subsubsection{AORTIC PHV'S}

The development of aortic PHV's has not been without problems. A multicenter 30-day prospective study of CoreValve implantation in 86 octogenarians was published in 2007. [66] The authors reported an overall $88 \%$ success rate and a $75 \%$ reduction in the 30 day mean transvalvular pressure gradient. However, they reported a $34 \%$ occurrence of increase in regurgitation and a $66 \%$ occurrence of no change in regurgitation post-implantation. The mortality rate was $12 \%$, and the major adverse cardiac and cerebral event (MACCE) rate was 26\%. Complications reported included inaccurate device deployment, perivalvular leakage, stroke, death, and cardiac tamponade due to ventricular perforation via the guidewire. Interventional therapies administered during the PHV procedures included BAV, rapid pacing, extracorporeal circulatory 
support, and total peripheral cardiopulmonary bypass. The delivery catheters in all cases were 21 or $18 \mathrm{~F}$ and the approach was retrograde via iliac, subclavian or femoral arteries. There was no control for the study; therefore conclusions about device efficacy could not be drawn.

A single center study of 33 cases of Sapien PHV implantation into octogenarians was reported in 2006. [64] Data from 30-days and 6-months post-implantation were evaluated. In terms of procedural approach, $79 \%$ were antegrade and $21 \%$ were retrograde. The antegrade approach had an $85 \%$ success rate, and the retrograde approach had a $57 \%$ success rate. The 30 -day mortality rate was $18 \%$, and the 6 -month mortality rate was $37 \%$. As of the date of the publication $33 \%$ of the study patients were still living. There was an overall reduction in transvalvular pressure gradients of about $75 \%$ and mild to severe regurgitation post-implantation and zero to moderate regurgitation at 6 -months. The delivery catheter was $24 \mathrm{~F}$ in all cases.

Berry et al. published a case report in 2007 detailing their experience with CoreValve. [51] The patient died during the procedure as a result of a stroke caused by a piece of plaque dislodged from the catheter introduction site in the left external iliac artery which occluded the left subclavian artery. The delivery catheter was $21 \mathrm{~F}$. This case demonstrated the need for rigorous peripheral vascular studies prior to approval of aortic PHV trial candidacy.

\subsubsection{PULMONARY PHV'S}

The less dramatic consequences of pulmonary valve failure have allowed pulmonary PHV development to outpace aortic PHV development. A 2005 report 
detailed the clinical outcomes of 59 pulmonary PHV recipients. [67] The study spanned 36 months. In contrast to the clinical trial participants in aortic PHV trials, pulmonary PHV candidates are generally not critically ill and are pediatric. Study inclusion criteria were the presence of a surgically repaired RVOT, symptomatic dysfunction of the RVOT, right ventricular hypertension, dilatation or failure, and pulmonary insufficiency. In other words, the PHV procedure was not last resort as in the case of aortic PHV's and was indeed voluntary. The average study participant was 16 years old and weighed 56 kg. $78 \%$ of them had homograft RVOT's. A $98 \%$ initial success rate was reported with zero deaths. The results showed a $22 \%$ drop in RV pressure, a $41 \%$ drop in RVOT pressure gradient, and a $27 \%$ increase in PA diastolic pressure. Regurgitation was no more than mild in all cases. At 36 -months $70 \%$ of the PHV's were still functioning. Complications included stent dislocation from the guide wire, homograft dissection via balloon oversizing, detachment of the tip of the delivery catheter, local bleeding, in-stent stenosis caused by a "hammock effect" in early iterations of the Melody PHV where the entire vessel was not sutured to the stent, stent fracture, late stent embolization (9months) into the PA, residual stenosis, intravascular hemolysis, and endocarditis.

Melody PHV stent fracture has become such a concern that a report investigating its occurrence and risk factors was published in 2007. [54] In a retrospective study of 123 Melody recipients, there was a $21 \%$ rate of stent fracture. Regression analysis showed a statistically significant relationship between RVOT type, PHV recoil upon dilatation, RVOT calcification, and stent fracture. $49 \%$ of patients who experienced stent fracture required reintervention versus $6 \%$ who did not have fractured stents. Stent fracture grades correlated to restenosis, i.e. fracture "type I" was associated with less than or 
equal to one stent strut failure and had little consequence, but "type II" was associated with a loss of stent integrity which would lead to restenosis. Furthermore, the rigid platinum-iridium stent and the PA-sternal juxtaposition likely contributed to early stent fracture. Additionally, Bonhoeffer et al. reported the first engineering analysis of their PHV in 2007. [90] A biomechanical engineering team in Italy performed finite element analysis of the Melody stent. Unsurprisingly, they found high stress concentrations at the weld locations in the stent. Their model predicted fracture at those locations which correlated with in vivo findings.

\subsection{PHV TECHNOLOGY ANALYSIS AND FUTURE GOALS}

All of the aforementioned complications add up to ample opportunity for technological investigation and improvement. Biologic valves are known to have short useful lives. An alternative material will need to be developed to increase PHV durability. Stent deployment, fixation and integrity will have to be improved via rigorous study of PHV mechanics. The profile of PHV's will have to be reduced in order to accommodate smaller patients and guarantee safe peripheral delivery. Distal aortic protection against emboli may need to be developed and PHV retrievability and repositionability will be helpful features. More rigorous in vitro and animal testing will be required to optimize PHV designs. Effective imaging techniques and a preprocedural patient study protocols will need to be developed, which could lead to new imaging uses or technologies. [31,41,47,48,50,53,76,77,78,79,80,81,82,83]

The physiological and engineering implications of PHV's have only recently begun to be investigated. A 2007 study reported results of exercise tests in 17 patients 
who had received a Melody valve. [57] The results showed that reduced pulmonary regurgitation via PHV implantation improved the efficiency of right ventricular ejection, left ventricular filling, and increased cardiac output. A 2004 paper published results of an investigation into the effect of Sapien valve implantation on left ventricular function. [84] The results showed kinetic improvement in LV function via Doppler imaging.

Stock et al. reported the successful use of tissue-engineering techniques to prevent damage to tissue PHV's caused by the delivery process. [56] They seeded acellular matrices in pulmonary PHVS and cultured them in a dynamic bioreactor for 16 days. Then they crimped and deployed the valves and performed histological studies of the tissue. Their results showed that the tissue engineered valves exhibited almost no tissue damage whereas the control exhibited extensive cellular damage. This finding could be useful in extending the durability of tissue PHV's.

Attmann et al. investigated the significance of several PHV delivery systems. [34] They identified key design requirements for PHV delivery systems including outer diameter, inner diameter, and total length versus usable length, flexibility, stiffness, force transmission, handling, and durability. They also reported the first histological study of a pulmonary PHV. [33] They microscopically examined tissue pulmonary PHV's after 3months of service in sheep. Their results showed calcification of the tissue valve primarily in the wall.

PHV technology has been proven to be feasible by these pioneering researchers and inventors, and there is a demonstrated demand for the technology. The future of heart valve prosthetics will likely include PHV's for a while to come. 
The work described herein takes the PHV concept a step further into the future. Tissue valves have well known limitations and the ill effects of using tissue valves in PHVs have just started to become elucidated. An artificial valve with equal or superior hydrodynamics to and superior durability to tissue remains the best choice for PHVs. By incorporating state-of-the-art polymers with a functional novel PHV stent design, this work will move the technology closer to commercial fruition and possibly lead to a breakthrough in polymeric heart valve technology.

\section{PRELIMINARY PHV WORK AT FIU}

Work on artificial aortic PHV's in the FIU CVEC was initiated by a graduate student, named Fernando Jaramillo Arbeláez (FJA), who was awarded a Master's Degree in Biomedical Engineering in 2005 for his efforts. [69] His aim was to prove the feasibility of an artificial aortic PHV. His goals focused on PHV fixation, crimpability, leaflet construction, and hydrodynamics. Early on he decided to pursue a Nitinol stent as a frame for his PHV design. His initial prototype used a fixation method that was intended to clip onto the native leaflet for backflow retention and expand into the left ventricle for forward flow retention. He also employed a leaflet design that resulted in three ellipsoidal cups mounted within a stent, which he called "double coaptation leaflets". He reported that preliminary in vitro tests of his leaflet design were comparable to the function of a $25 \mathrm{~mm}$ diameter bileaflet St. Jude mechanical valve. He also endeavored to create a suitable leaflet material by experimenting with different fabrics and SIBS coatings. Later he experimented with another leaflet design, which he called "central coaptation leaflets". It is apparent that his goal was to design leaflets that had 
the shape of natural leaflets. Furthermore, he created several more stent designs by varying the number of turns in the wire and altering the method of fixation. According to his conclusions the design he called "MCB", central coaptation leaflets and stent with forward and backward flow retention hooks, was his best performing design. He also created a delivery device for the in vitro deployment of his PHV's into a porcine aorta. Moreover, he created a device for the mounting of said porcine aorta into the Vivitro Systems, Inc. Left Heart Simulator (LHS) for hemodynamic testing of the natural porcine aortic valve and his PHV prototypes. His final prototypes were crimpable to less than 24 F. Ultimately, he created a functional method for manufacturing Nitinol stents and proved the concept of an artificial PHV. His work was the starting point for the work described herein.

\section{RESEARCH PURPOSE}

The purpose of the research performed here was to move the development of an artificial aortic PHV forward through the feasibility stage of development by answering the following questions:

1. What is a superior functional design for an artificial aortic PHV?

2. What are the appropriate materials for said PHV?

3. What constitutes a novel functional PHV delivery system design suitable for the deployment of the novel PHV design?

4. Can an artificial aortic PHV produce hydrodynamics comparable to commercially available heart valve prosthetics? 
5. Can the PHV be tested for deliverability and hydrodynamic parameters via a controlled and repeatable in vitro test method?

The long term goal is to create an artificial PHV and delivery system suitable for further development and movement into animal testing and eventual human testing.

\section{SPECIFIC AIMS AND OBJECTIVES}

The feasibility of the novel PHV in development was demonstrated by conforming to the following specific aims:

1. Quantitative in vitro hydrodynamic characterization of a novel artificial aortic PHV.

2. Quantitative in vitro characterization of the PHV's static mechanical properties.

3. Quantitative characterization of PHV crimpability.

4. Quantitative and qualitative comparison of a novel PHV to existing PHV empirical data and designs.

5. Qualitative characterization of a novel PHV delivery system.

6. Quantitative and qualitative characterization of an in vitro PHV testing model.

Each of these specific aims provided input into the design of the PHV and its delivery system and feedback concerning their performance. Aim 1 indicated the basic feasibility of the PHV design via quantifying its in vitro hydrodynamic performance, e.g. pressure drop across the valve, flow rate, and mean systemic pressure. Aim 2 showed whether or not the PHV remained in place under a variety of conditions once deployed into the test system, i.e. it exhibited no migration in situ. Aim 3 showed whether or not the PHV design could be crimped to a specified diameter for delivery. Aim 4 showed whether or 
not the PHV in development is performing comparably to competitor designs and the "gold standards". Aims 1-4 together illustrated the feasibility of a novel PHV. Aim 6 showed the feasibility of a PHV delivery system, and aim 7 showed the feasibility of an in vitro test model.

The objectives of the research performed as defined by the investigator included five major design requirements of aortic PHV's:

1. Crimpable to $24 \mathrm{~F}$ or less

2. Produces a mean pressure drop of $10 \mathrm{mmHg}$ or less

3. Allows an average of $5 \%$ or less leakage of flow

4. Allows no migration in situ

5. Provides normal physiological pressures and flow rates at a given heart rate, e.g. $90 \mathrm{mmHg} \mathrm{MAP}$ and $5 \mathrm{~L} / \mathrm{min} \mathrm{CO}$ at an HR of $70 \mathrm{bpm}$.

An in vitro test model was developed such that PHV prototype testing could be conducted in a uniform, repeatable, and controlled manner, and a catheter could be inserted into the LHS during operation for PHV delivery.

These objectives were justified by the available related clinical and scientific evidence. Equation 1 illustrates the direct relationship between the pressure drop across the aortic valve and the amount of work done by the heart. An increased work load caused by stenosis can cause hypertrophy of the heart and eventual heart failure due to decreased compliance in the wall of the heart. Conversely, increased leakage flow eventually decreases the amount of work that the heart can perform by decreasing SV, which would also lead to cardiac failure by an enlargement and thinning of the heart walls causing a decrease in contractility. Additionally, commercially available heart 
valve prosthetics produce pressure gradients of less than $8 \mathrm{mmHg}$ and leakage flow of less than $4 \%$. [3] Furthermore, table 1 shows that mild stenosis of the natural aortic valve is clinically defined as having a pressure gradient of $25 \mathrm{mmHg}$ or less, and mild regurgitation $(1+)$ is defined as having $30 \%$ or less leakage flow. [68] Moreover, experiments show that having less than $5 \%$ regurgitation is necessary for complete valve closure during diastole. [10] In general, the maximum dilated diameter of a healthy femoral artery is $8 \mathrm{~mm}$ or $24 \mathrm{~F}$. However, early PHV retrograde delivery reports indicate that peripheral vascular disease can compromise this approach. [51] Therefore, using the smallest profile delivery catheter possible would minimize risks of embolism and vascular injury. Finally, a PHV would simply be useless if it could not aid in sustaining a proper arterial pressure and cardiac output at a normal heart rate.

\section{METHODS}

\subsection{OVERVIEW}

First, the project was defined, and then specific aims and goals of the project were identified. The work began with the replication of FJA's test methods using the original MCB PHV. Critical evaluations of that test experience lead to new insights and directions. Concurrently, a thorough literature review was conducted and a new PHV concept began to form. PHV prototyping and testing then began, and the design process described in the following section was employed. Finally, an objective analysis of the verification test data coupled with a qualitative analysis of the prototypes, models, and methods lead the conclusions reported. 
The design process employed for this research followed FDA Regulation 21 CFR 820.30 Subpart C; Design Controls (figure 10).

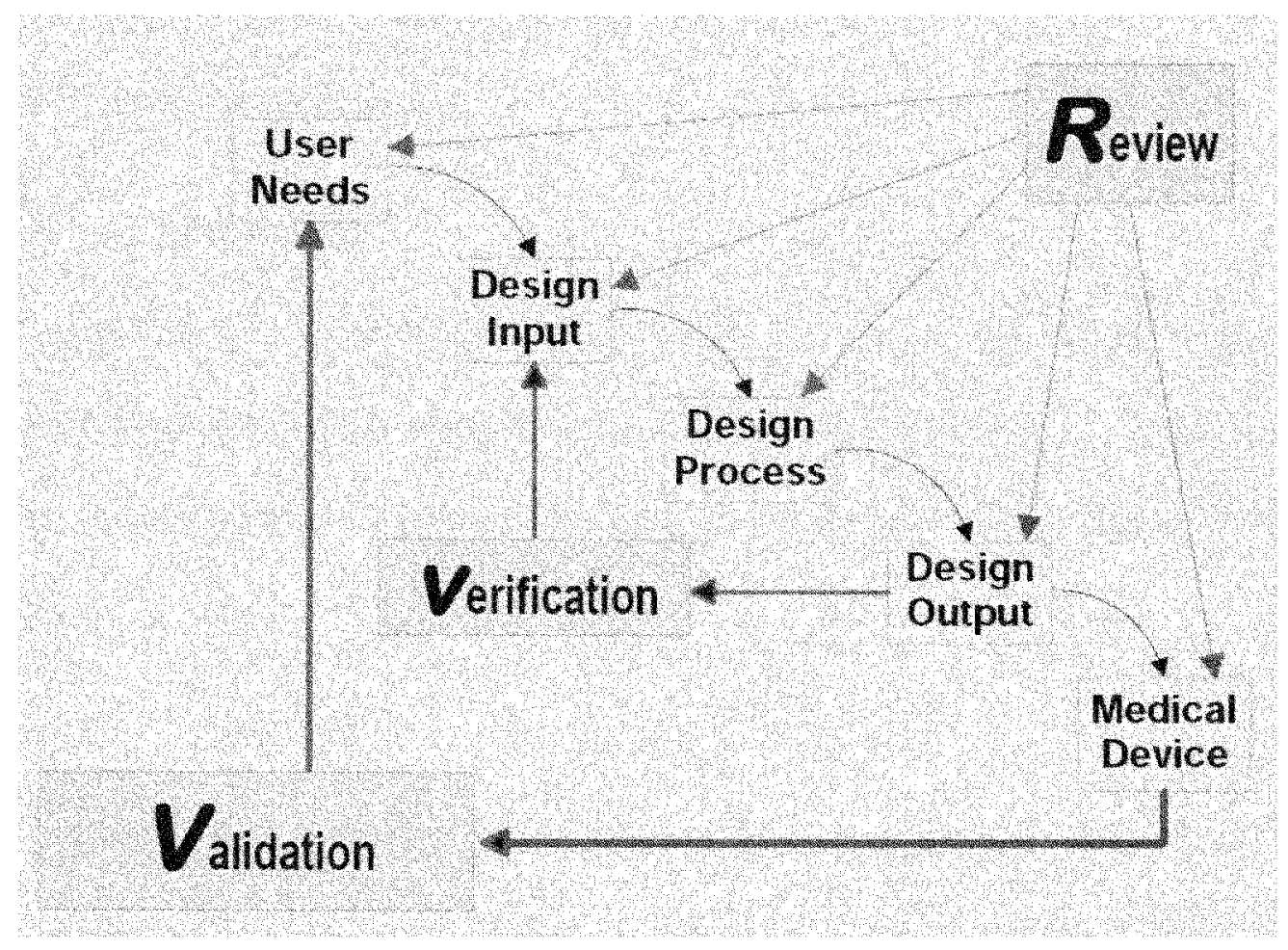

Figure 10; FDA 21CFR 820.30 Design Control Guidance Diagram.

In accordance with 820.30 a design history file (DHF) has been maintained, which included the laboratory notebooks and all other pertinent documentation generated via the design process. Validation testing was not performed because a finished product ready for transfer to manufacturing has not yet developed. However, verification testing was performed at each stage of the design process, which fed data back into the process that aided in improvement of each prototype. 
As presented earlier in this report, there is a clearly demonstrated demand for PHV technology by interventional cardiologists and patients seeking less traumatic procedures for the treatment of valvular heart disease. This market need has been identified via extensive research.

\subsection{RESEARCH}

The history of PHV technology has been extensively researched via academic journal database queries, scientific text reviews, clinician interviews, and general internet queries. A compilation of the researched information has been utilized to form a solution concept.

\subsection{CONCEPT}

A PHV concept has been formed based upon the research. The concept draws upon known successes and complications concerning currently reported PHV designs. Cost and manufacturability influenced the concept solution as well.

\subsection{DESIGN AND FABRICATION}

\subsubsection{THE STENT}

The stent was the frame or support structure for the valve. It also provided for exclusion of the diseased native valve and fixation of the PHV in the aortic root. A two stacked stent design similar to the first PHV built and tested by Andersen et al., was employed because it allowed economical and quick fabrication of prototypes in house. The shape of the stent was based upon the CoreValve concept, using an oversized distal 
stent, and in turn friction, as the primary fixation feature. Fixation of the PHV was designed to depend upon over-sizing of the distal stent by at least $20 \%$. Hooks were abandoned early on due to their hindrance of delivery and potential to cause trauma to the aortic wall. The use of two stents in the proximal portion of the stent where the valve resided was chosen after several prototype tests revealed the need for stiffening of that section. For the distal fixation stent, tear-drop bends were created at each turn that served to stiffen the stent. [59] The added stiffness in each joint reduced the need to have more bends in the wire. Therefore, the number of wire bends exposed to the blood was minimized because each part of the wire that resides perpendicular to the flow of blood tends to induce neointimal hyperplasia and thrombosis. [94] U-shaped bends were placed in the proximal stent to facilitate crimping and because the bulk of the surface was covered by the leaflet material, and eventually two stents were utilized for added stiffness. In contrast to the Andersen valve, our PHV had and over-sized fixation stent with geometry that maximized stiffness, and in contrast to the CoreValve design, our PHV exposed less stent strut area to the blood and provided larger spaces for the passage of catheters into the coronary arteries.

Initially, the stent was fabricated from commercially available stainless steel wire for economy and rapid prototyping. A wooden peg board was used to bend the wire into the desired shape as shown in figure 11. The ends of the wire were joined using stainless steel hypodermic tubing and super-glue (figure 12), and the stent was expanded using a 12 F Bard urinary catheter balloon $(5 \mathrm{ml})$. The initial Nitinol wire stent was fabricated using a ceramic peg board and a small butane torch (figure 13). 


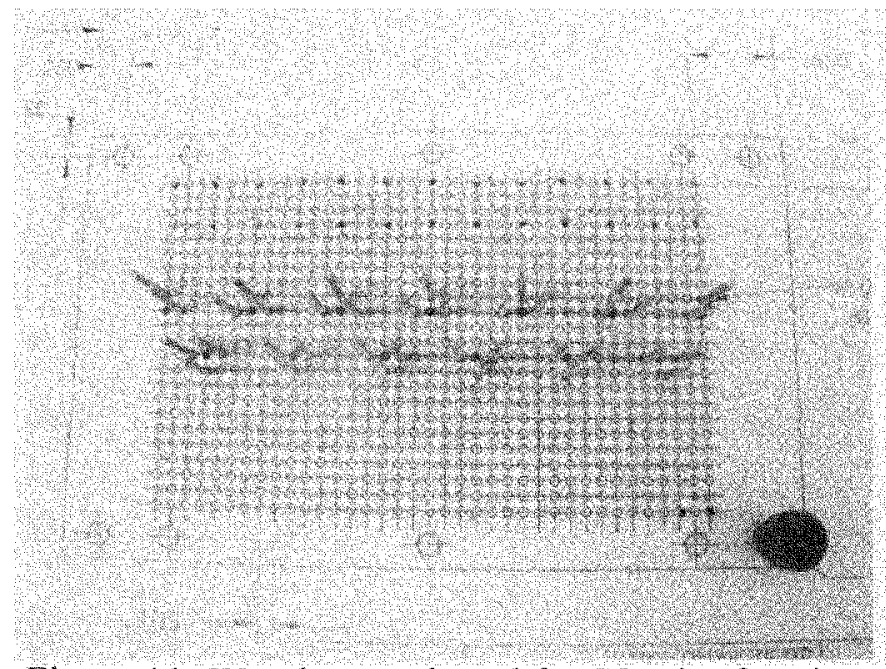

Figure 11: Wooden peg board for SS wire forming.

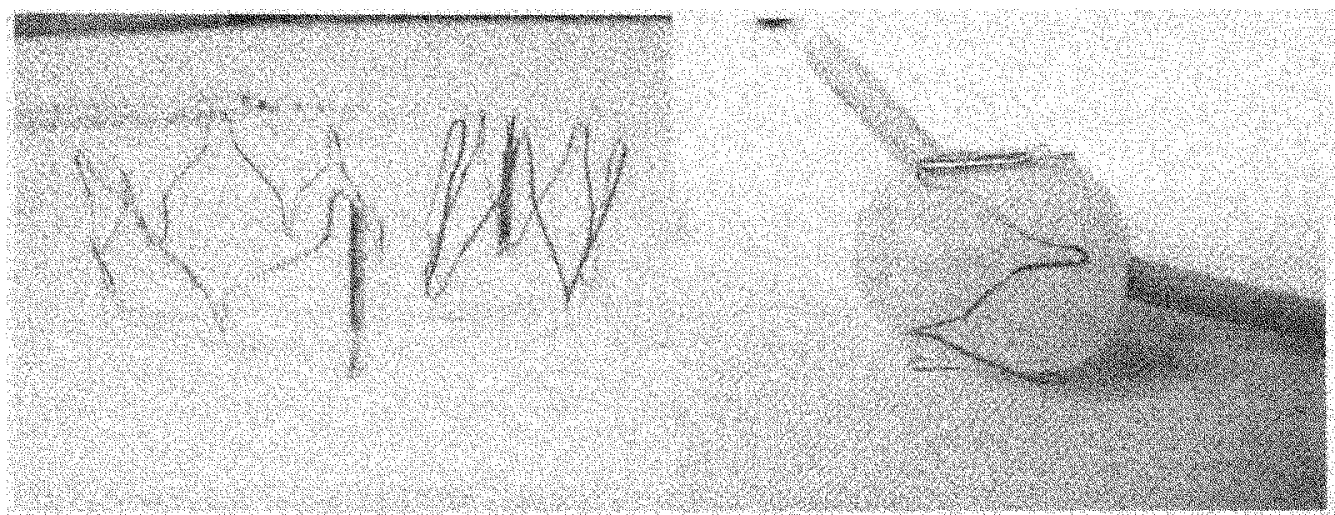

Figure 12: Upper SS wire with hypo-tubing, and lower urinary catheter balloon used to expand the stainless steel stent.

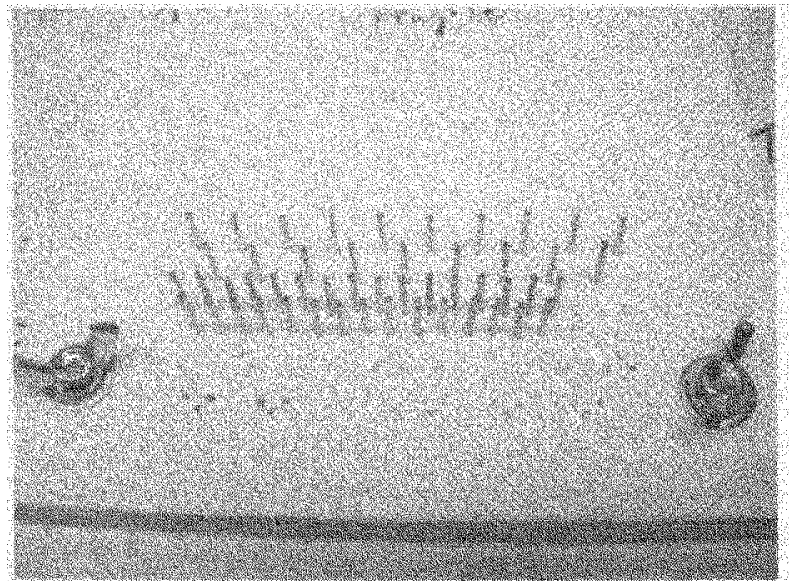

Figure 13: Bench-top Nitinol wire forming ceramic peg board. (The board is missing some dowel pins in this photo.) 
The Nitinol wire was bent in the pattern of the pegs and the torch was used to anneal the wire so that it would retain the desired shape. The process was imperfect and produced less than ideal prototype stents. However, the method was used for speed and economy.

The final version of the Nitinol stent was manufactured using a method similar to Jaramillo's, Steel plates were fabricated with holes drilled in a predetermined pattern, and 1/16 inch diameter hardened steel pins were press-fit into the holes (figure 14). A top plate was bolted onto the bottom peg-board plate in order to constrain the wire while heating. The assembly was heated to $500^{\circ} \mathrm{C}$ and soaked at that temperature for 5 minutes. Then the assembly was removed from the furnace and water quenched. The ends of the Nitinol wires were joined in the same manner as the stainless steel wires using stainless steel hypodermic tubing (figure 15). The stents produced in this manner were of much higher quality than those produced using the butane torch. The diameter of the nitinol wire varied with each prototype until an optimal diameter was found via verification tests.

Later, a need for larger diameter stents arose and the annealing plates were redesigned (figures 16 and 17). Anchors were incorporated into the new design.

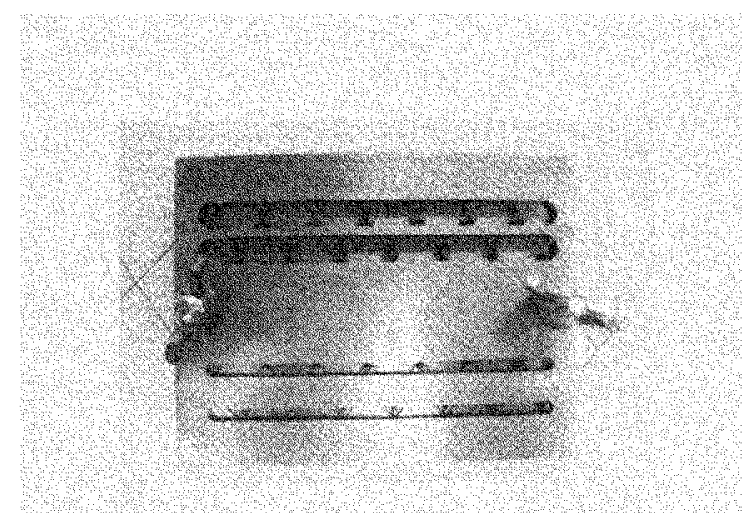

Figure 14: Steel plate for Nitinol wire annealing in a $500^{\circ} \mathrm{C}$ furnace. 


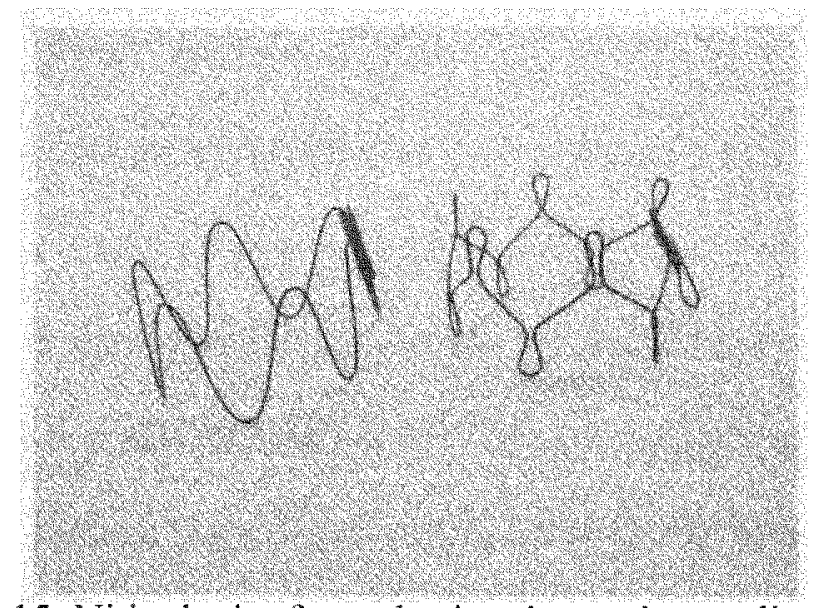

Figure 15: Nitinol wire formed using the steel annealing plates.

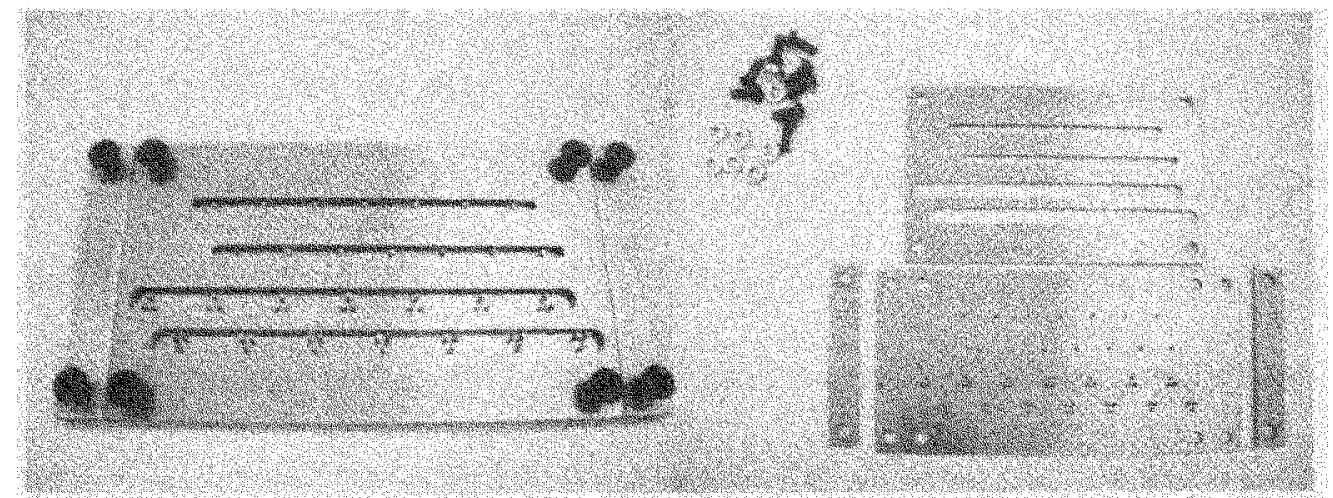

Figure 16: Second generation nitinol wire annealing plate. This allowed for larger diameter stents to be manufactured.

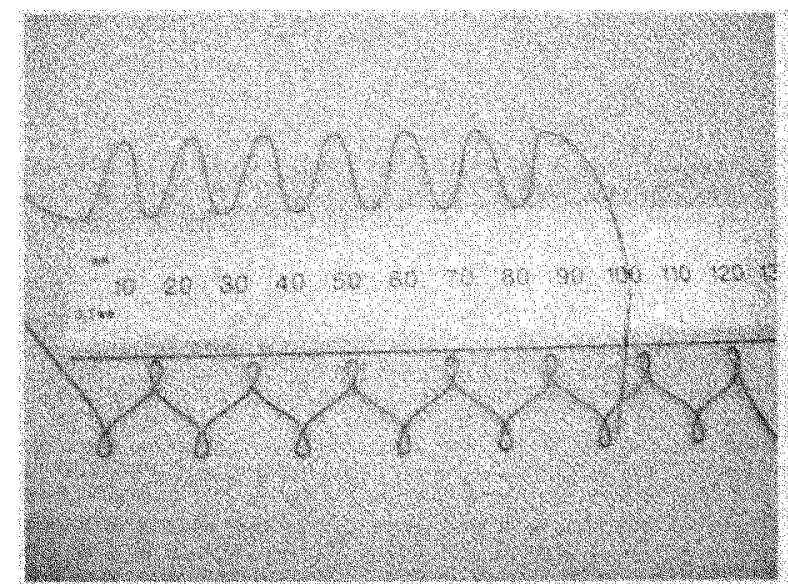

Figure 17: Nitinol wire after annealing in the second generation fixture. 


\subsubsection{THE VALVE}

The valve was designed to function as the natural aortic valve, that is, like a collapsing tube. An extensive review of human anatomy and physiology was used as a primary input to this design. The material used for the valve had been predetermined, but material shape and thickness were manipulated during the design process.

The leaflet material was manufactured using a method created by Siobhain Gallocher. A $3 \times 3$ inch sheet of Bard Peripheral Vascular P04081 Polyester Fabric Style 6102 was constrained onto an aluminum drying plate, and $7-8 \mathrm{ml}$ of liquefied SIBS (Innovia, LLC.) was poured onto the mesh, and the composite material was allowed to cure at room temperature under a fume hood for 48 hours (figure 18). The SIBS polymer was provided in the form of small solid pellets and was liquefied by adding toluene $15 \%$ by mass, i.e. the mixture was $15 \%$ SIBS and $85 \%$ toluene. [58] Commercially available aluminum foil was used as a gasket between the fabric and the drying plate in order to provide a space for SIBS to coat the surface of the polyester fabric in contact with the mirror finish base of the drying fixture.

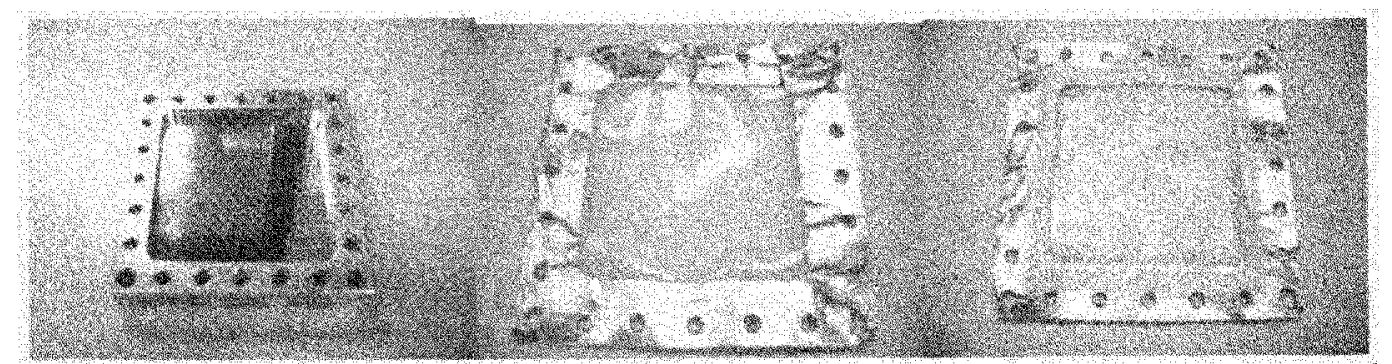

Figure 18: Leaflet composite material and drying plate by Gallocher. One side of the leaflet material turns out very smooth (ventricular side-bottom left) and the other is rougher (aortic side-bottom right) much like the natural aortic valve. 
The resulting composite had a thickness between 200 and 250 microns depending on the volume of SIBS-toluene mixture added to the polyester fabric. Once the SIBS had cured, the composite sheet was removed from the plate and cut to the desired valve dimensions.

The test conduit diameter was $19 \mathrm{~mm}$, so the leaflet material was cut to form a cylinder of equal diameter. The length ( $L$ ) of the leaflet material was ideally $L=\pi d \approx 60$ $\mathrm{mm}$, where $\mathrm{d}$ was the conduit diameter. The height $(\mathrm{H})$ of the cylinder (valve) was calculated using the formula $H=r(1.245) \approx 12 \mathrm{~mm}$, where $\mathrm{r}$ was the radius of the conduit. The resulting $H$ value was considered ideal. The constant 1.245 was the mean value of the $\mathrm{H}$ multipliers extracted from anatomical studies cited in Thubrikar 1990, i.e. if $r=1$, then $H=1.245$. [10] The height of the stent (h) was approximately $h=2 \mathrm{H} \approx 24 \mathrm{~mm}$. A 19 mm diameter was chosen for the PHV prototype, because it matched the diameter of the aortic root of a small adult human and a sheep, which was the preferred test animal due to its blood coagulation characteristics and slow growth. A purse-string style stitch was used to join the overlapping ends of the fabric in order to form a cylinder (figure 19).

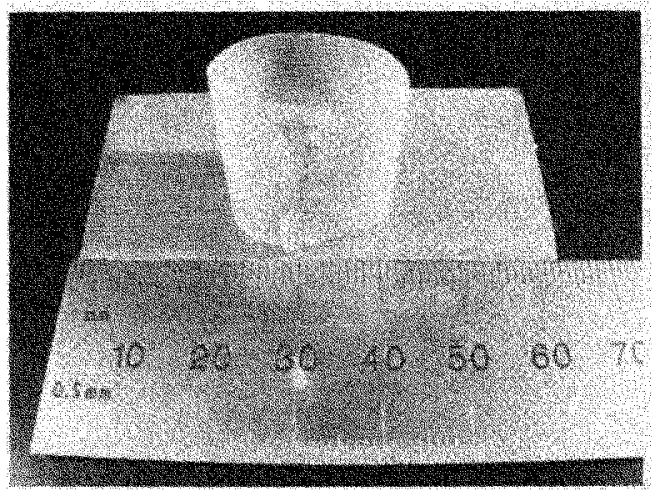

Figure 19: Dacron-SIBS composite leaflet material. Cylindrical form used for valve formation shown on top of a flat composite sheet. Scale is in centimeters. Notice the purse-string suture pattern used to form a cylinder. 
A detailed protocol of PHV construction is located in the appendix. The valve material was sutured to the stent via braided polyester suture (Ethibond Excel 4-0). This suture was chosen because it has a high tensile strength, it resists breaking when flexed or knotted, and it is being used in the manufacture of commercially available abdominal aortic aneurysm (AAA) stent grafts. In lieu of welding, the suture was utilized to join segments of the stents as needed (figure 20). The final step in the process was the placement of one stainless steel ball bearing behind each of the three leaflets, and the placement of the PHV onto the $19 \mathrm{~mm}$ diameter mold spacer. This was performed in order to anneal the leaflets into the hemispherical shape of natural leaflets (figure 21). The assembly was placed inside a $60^{\circ} \mathrm{C}$ oven for about 2 hours for shape setting.

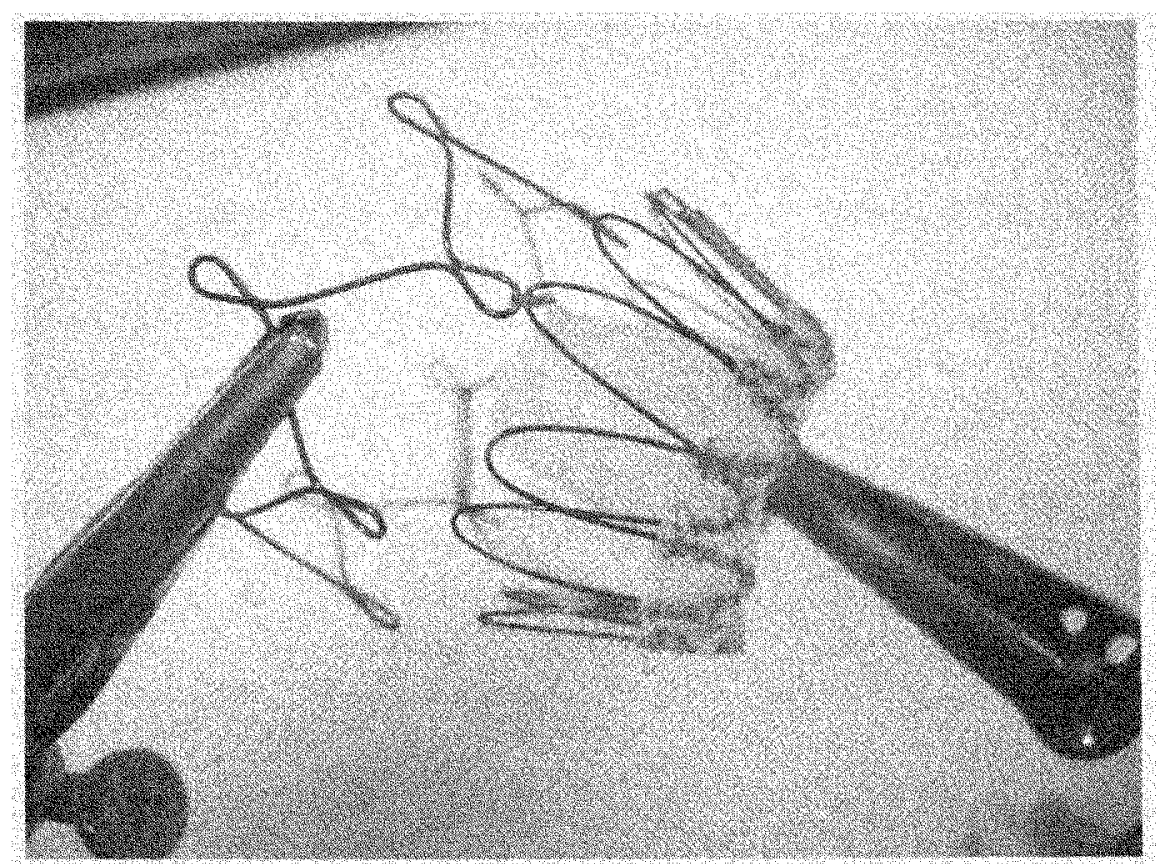

Figure 20: PHV shown during assembly. 


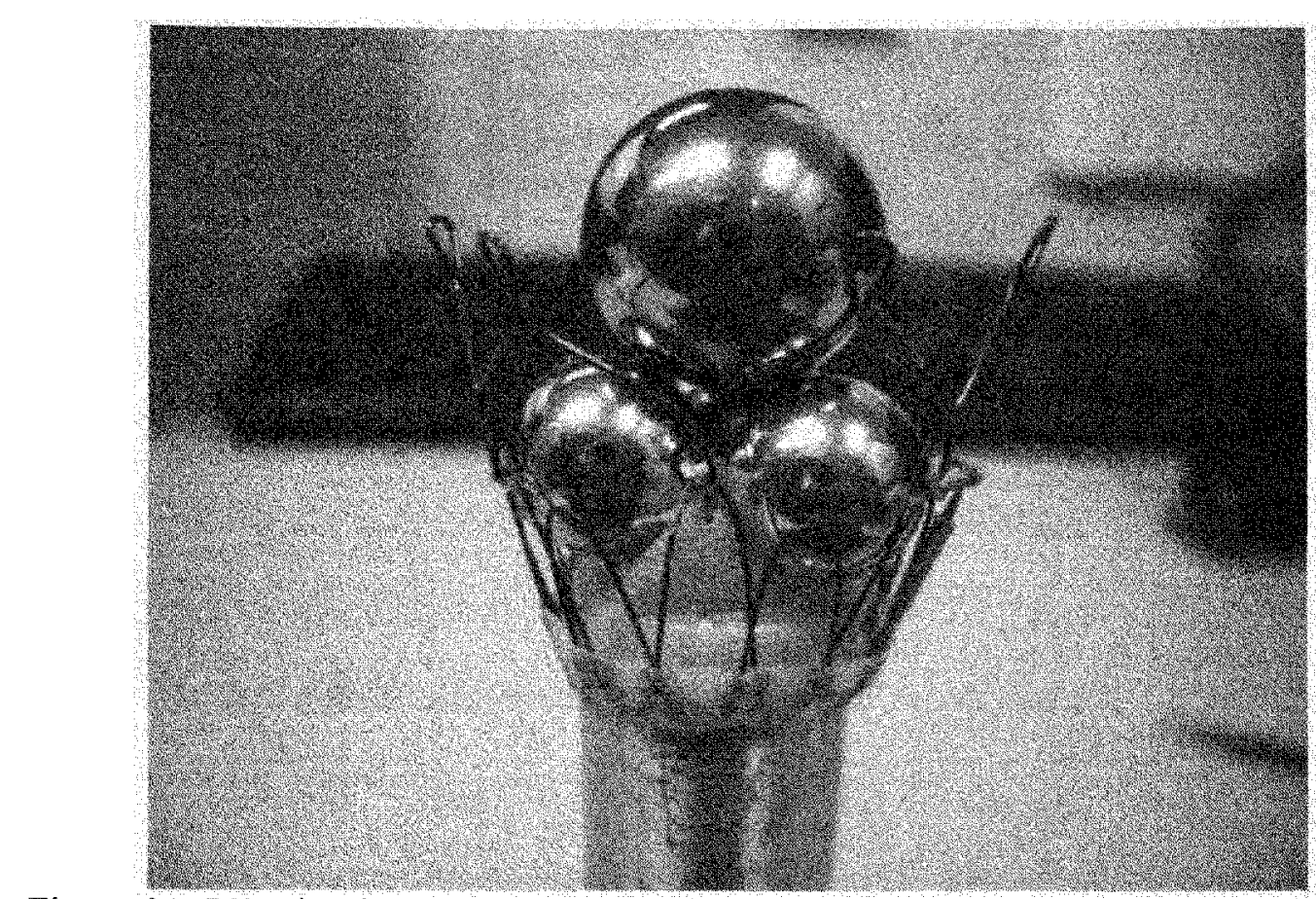

Figure 21: PHV in place on the $19 \mathrm{~mm}$ diameter SA mold spacer with $\mathrm{SS}$ ball bearings ready to be placed into the oven for annealing.

\subsubsection{THE DELIVERY SYSTEM}

It was determined via literature review that the PHV should be delivered retrogradely to the heart via peripheral arterial access. So a mechanism capable of doing so had to be designed. Ideally, a commercially available vascular catheter would have been be utilized, but there are very few available for delivering such large diameter devices. Therefore, a device had to be designed that could accommodate the PHV and at the same time fit into the lumen of an average large peripheral artery, e.g. the internal iliac artery. Additionally, a feature was added to the design that would enable "steering" of the catheter in situ so that the catheter could be centered in the aortic lumen prior to valve deployment. Finally, a mechanism was designed such that the retraction of the catheter sheath could be controlled with respect to the retraction distance. The catheter 
hand piece (Figure 22) was designed in SolidWorks SP3.1 2005 and machined from 6020 Aluminum. Catheter style FEP tubing was donated by Zeus Industrial Products, Inc. that had an OD of $24 \mathrm{~F}$ and an ID of $7 \mathrm{~mm}$ and a length of $1 \mathrm{~m}$. Internal support wires for the catheter were composed of Nitinol with 0.040 inch and 0.050 inch diameters were purchased from Small Parts, Inc. A Boston Scientific Jagwire 0.035 inch was used as a guide wire (figure 25). The catheter tip (figure 23) and stopper (figure 24) were designed in SolidWorks SP3.1 2005 and machined from white Delrin. The tip was designed with two lumens. One was drilled through at an offset angle with respect to the tip for passage of a guide wire and the other terminated inside the tip for anchoring of the internal support wire. The stopper was designed to fit tightly onto the Nitinol support wire so that the PHV could be crimped and inserted into the catheter between the tip and the stopper. The stopper functioned to hold the PHV in place while the sheath was retracted during deployment. It also was designed for attachment of the steering line and fixation of the central stopper wire. The steering mechanism employed a braided fishing line called Spectra ${ }^{\circledR}$ by Power Pro of $15 \mathrm{lb}$ test. The hand piece was assembled as shown in figure 22. The Jagwire passed through the guidewire slot on the top of the hand piece and on through the catheter tip, and the two internal nitinol wires were held in place via the set screw in the catheter hand piece. The spool was attached to the most distal portion of the catheter via Elmer's Krazy glue. (Ideally, the catheter and spool would be a single molded piece.) The fishing line was attached to the catheter stopper and threaded through the catheter lumen and into the steering rod of the hand piece. 


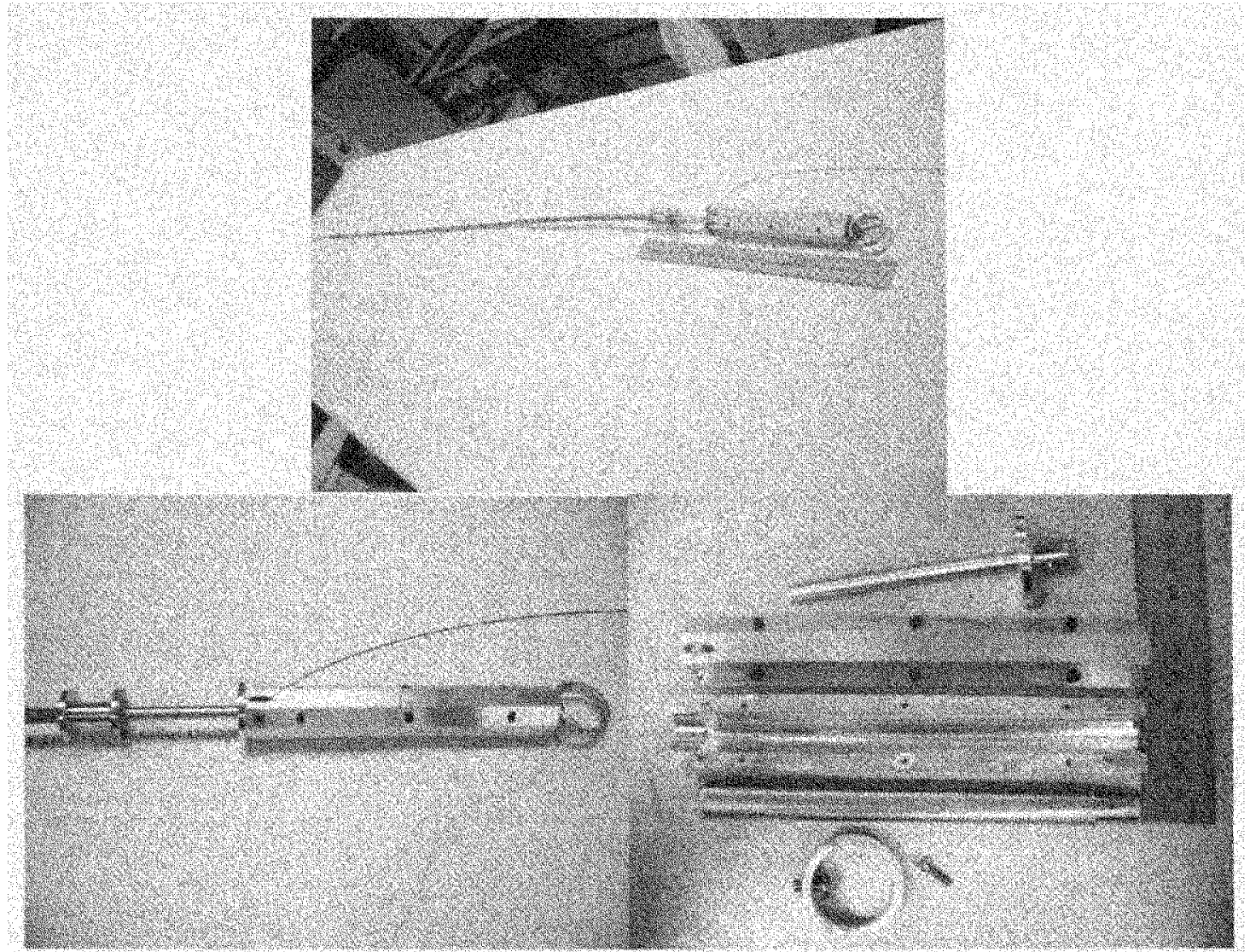

Figure 22: Deployment device hand piece. On the left, the Jagwire is shown protruding from the guide wire slot and the fishing line used to steer the catheter is shown hanging from the ring of the steering rod in the hand piece. On the right, the hand piece is shown fully disassembled.

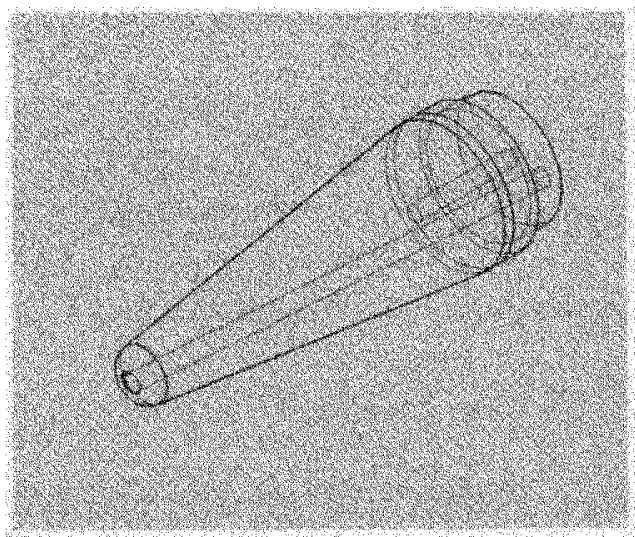

Figure 23: Catheter tip. 


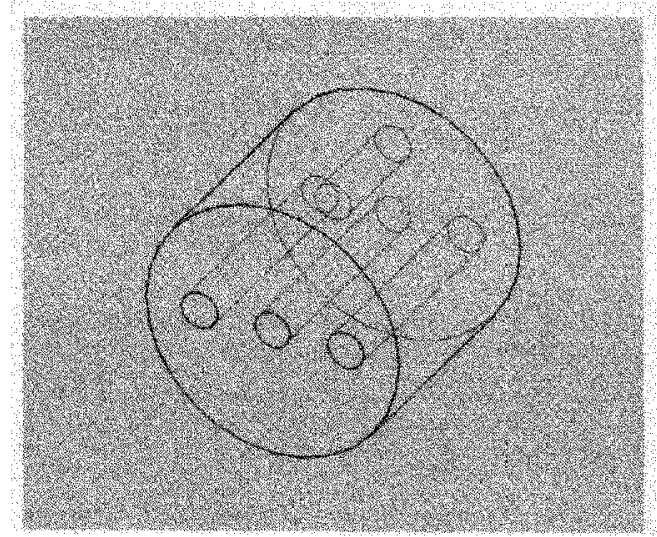

Figure 24: Stopper.

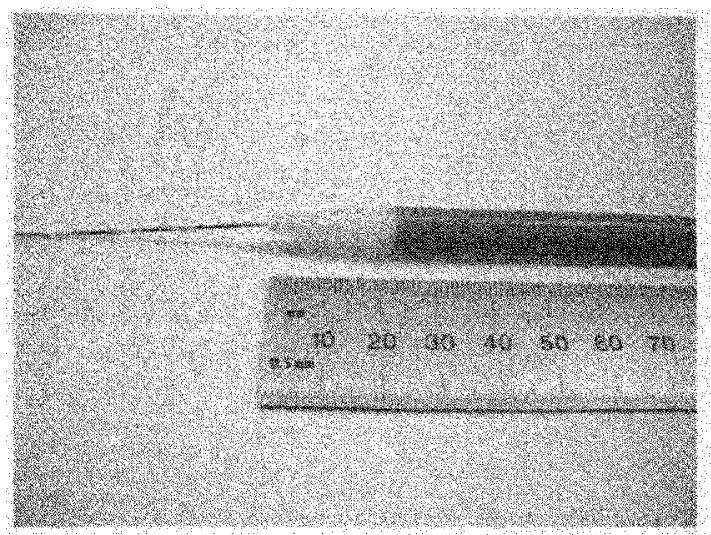

Figure 25: Zeus $24 \mathrm{~F}$ catheter tube with catheter tip and Jagwire.

\subsubsection{THE PHV CRIMPING TOOL}

It became apparent through the course of this work that crimping the PHV by hand for insertion into the delivery catheter was quite challenging. Therefore, a tool for doing just that was designed (figure 26). It was designed so that the PHV could be uniformly and cylindrically crimped to its smallest possible diameter or a diameter less than or equal to $7 \mathrm{~mm}$, which was the ID of the catheter sheath. Additionally, it was designed to hold the PHV in the crimped conformation once it was achieved. Finally, it 
was designed to allow crimping of the PHV over the internal support wire of the catheter and insertion of the PHV into the catheter sheath (figure 27).

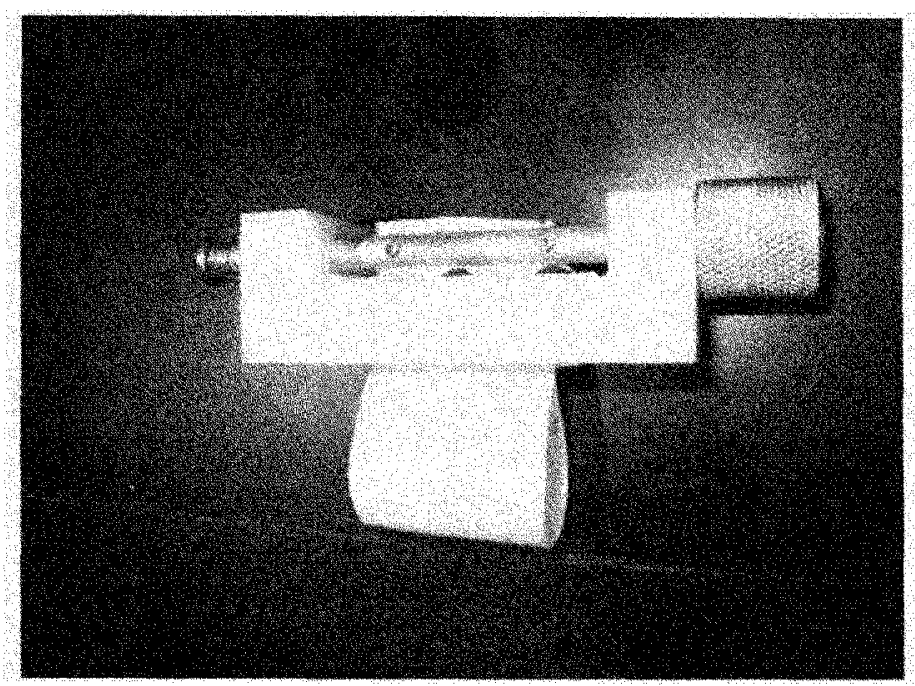

Figure 26: PHV crimping tool.

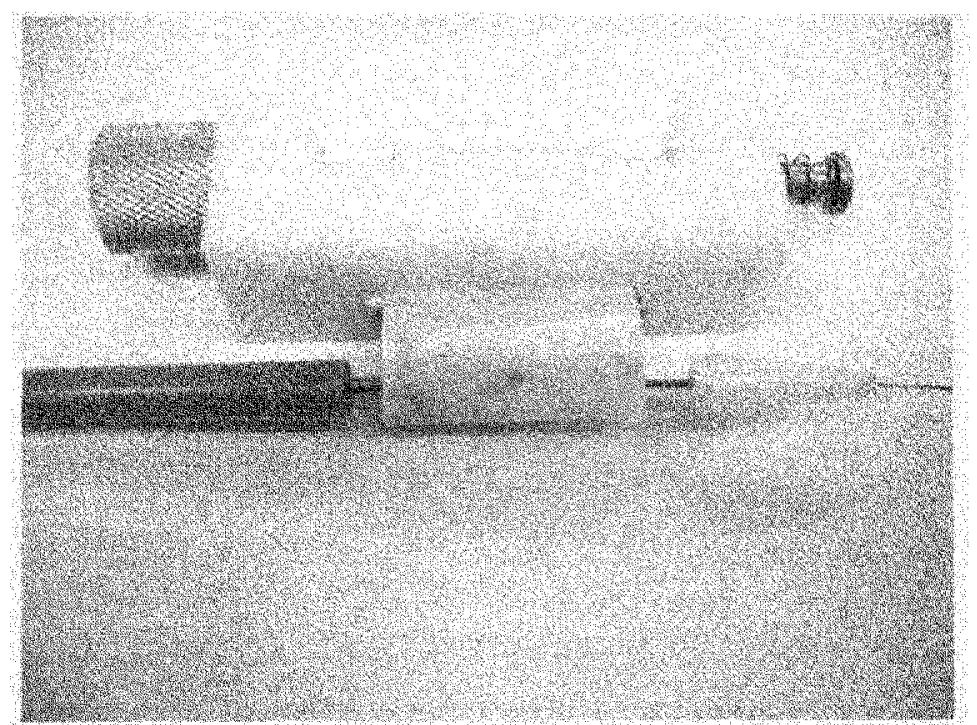

Figure 27: The PHV crimping tool shown with PHV inside the PTFE loop over the deployment catheter.

The crimping tool was machined from Delrin, aluminum and brass. The base block was Delrin, and spool shaft was brass and the spool knob was aluminum. These 
materials were chosen for ease of machining and economy. A 0.015 inch thick PTFE sheet was attached to the Delrin block and to the brass spool shaft and was used to crimp the PHV by winding the sheet around the shaft of the spool. As the spool was wound the loop of PTFE sheet on the opposite side of the block containing the PHV would squeeze the PHV into a smaller diameter conformation (figure 28). A ratchet-spring mechanism was designed to hold the spool at the desired level of tension.

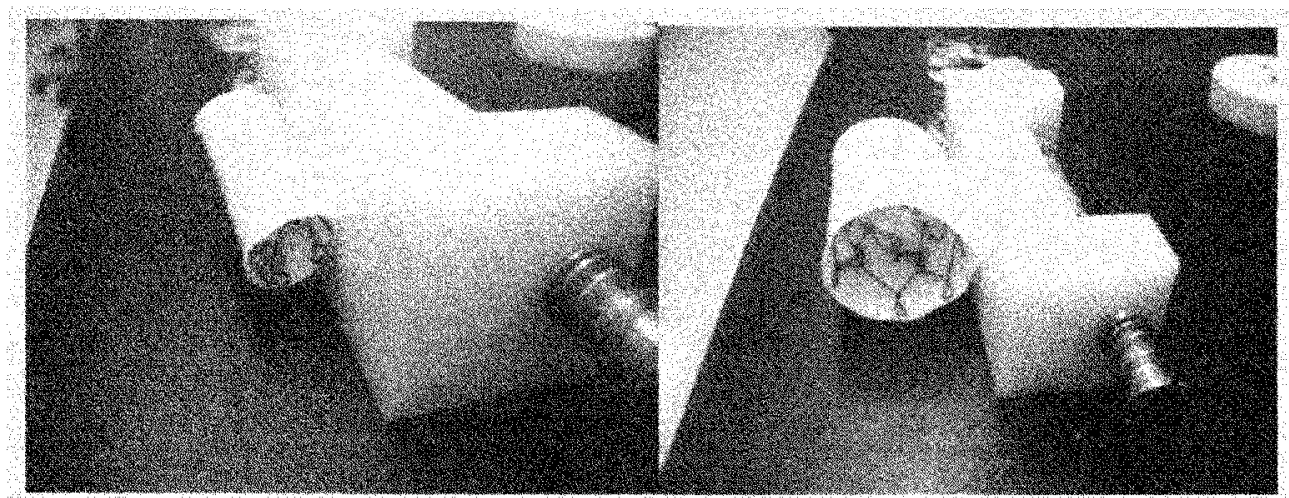

Figure 28: PHV crimping tool shown during the crimping process.

\subsubsection{THE FRENCH SIZER}

A French scale sizing block was designed using SolidWorks and machined from Delrin for the purpose of testing the crimpability of each prototype PHV (figure 29). It contained holes varying in size from $24 \mathrm{~F}-12 \mathrm{~F}$. Each hole had a chamfered opening to facilitate crimping and insertion. The depth of the holes was designed to be greater than the maximum length of the PHV so that complete crimp testing of the PHV prototype could be performed. A commercially available equivalent tool could not be found. 


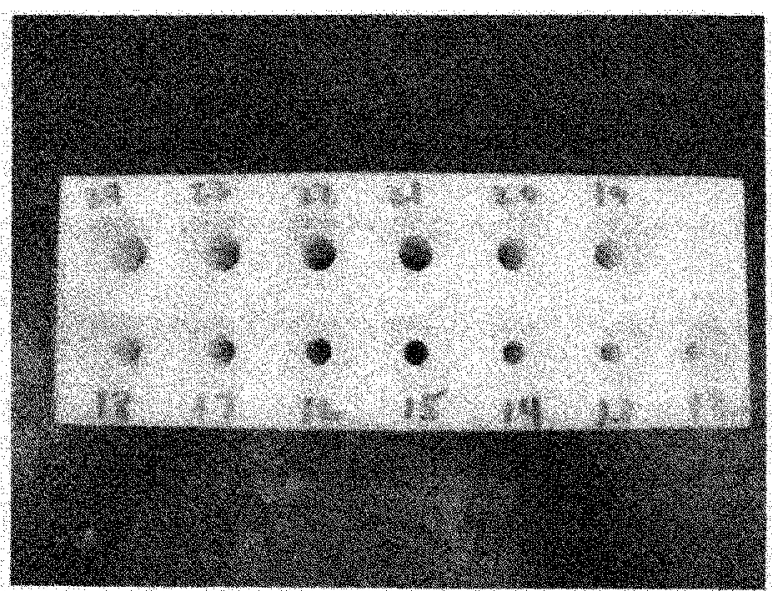

Figure 29: French scale block machined from Delrin.

\subsubsection{THE MODEL AORTA}

The Vivitro LHS in the CVEC was not designed and built for PHV testing, so the design of modifications to the system was essential to this work. It was determined during replication of the FJA experiments that the use of animal aortas inside the LHS introduced unnecessary complications to the project. Therefore, it was decided that a more consistent and less labor intensive method of testing the PHV prototypes in the LHS would be designed. It seemed a logical choice to create a silicone tube or aorta (SA) that could replicate the function of a real aorta without the variability and the labor intensity of the latter. The SA was designed to bypass and exclude the LHS characteristic (aortic) compliance chamber because in the end it was designed to be sufficiently compliant so as to replicate the built-in aortic compliance effects on the dynamics of the system. The internal diameter of the SA was designed to be $19 \mathrm{~mm}$. The tube was also designed to be transparent, which facilitated visualization of PHV performance during normal operation. The silicone rubber used was Sylgard 184 silicone elastomer by Dow Corning. It was initially mixed in a 10:1 ratio with the curing agent as specified by the manufacturer. The 
mixture was placed in a vacuum chamber to remove all air prior to pouring into the mold. Once poured into the mold, a central pin was inserted into the mold to create the $19 \mathrm{~mm}$ inner diameter. The silicone was allowed cure at room temperature for 48 hours.

Initially, a mold was designed to retrofit the FJA animal aorta apparatus with a silicone tube. The first generation silicone mold (figure 30) was machined from 304 stainless steel and PTFE shrink tubing was employed. The process for making and curing the first generation SA was imperfect, as illustrated by the tube shown in figure 30. The silicone contained many air bubbles and the wall thickness was not uniform. This prompted a return to the proverbial drawing board.

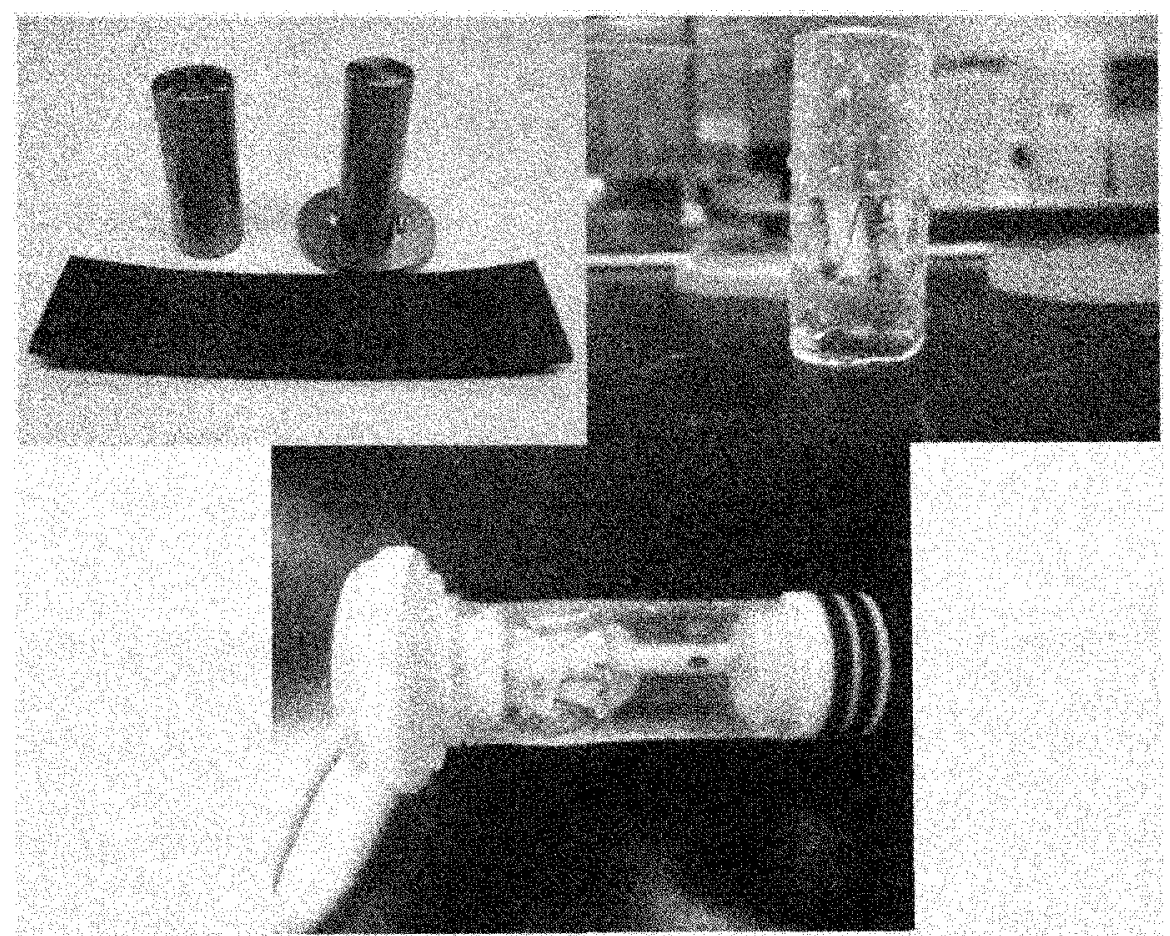

Figure 30: Silicone Aorta first generation mold with shrink tubing and SA 1.0 with FJAMCB by Claiborne deployed inside. Initially, the FJA mounting apparatus was employed. 
The second generation SA mold was designed to eliminate the need for the FJA mounting apparatus and to produce consistently uniform and air bubble free silicone aortas. It was designed as clam shell using Solid Works and was machined from 6020 aluminum (figure 31).

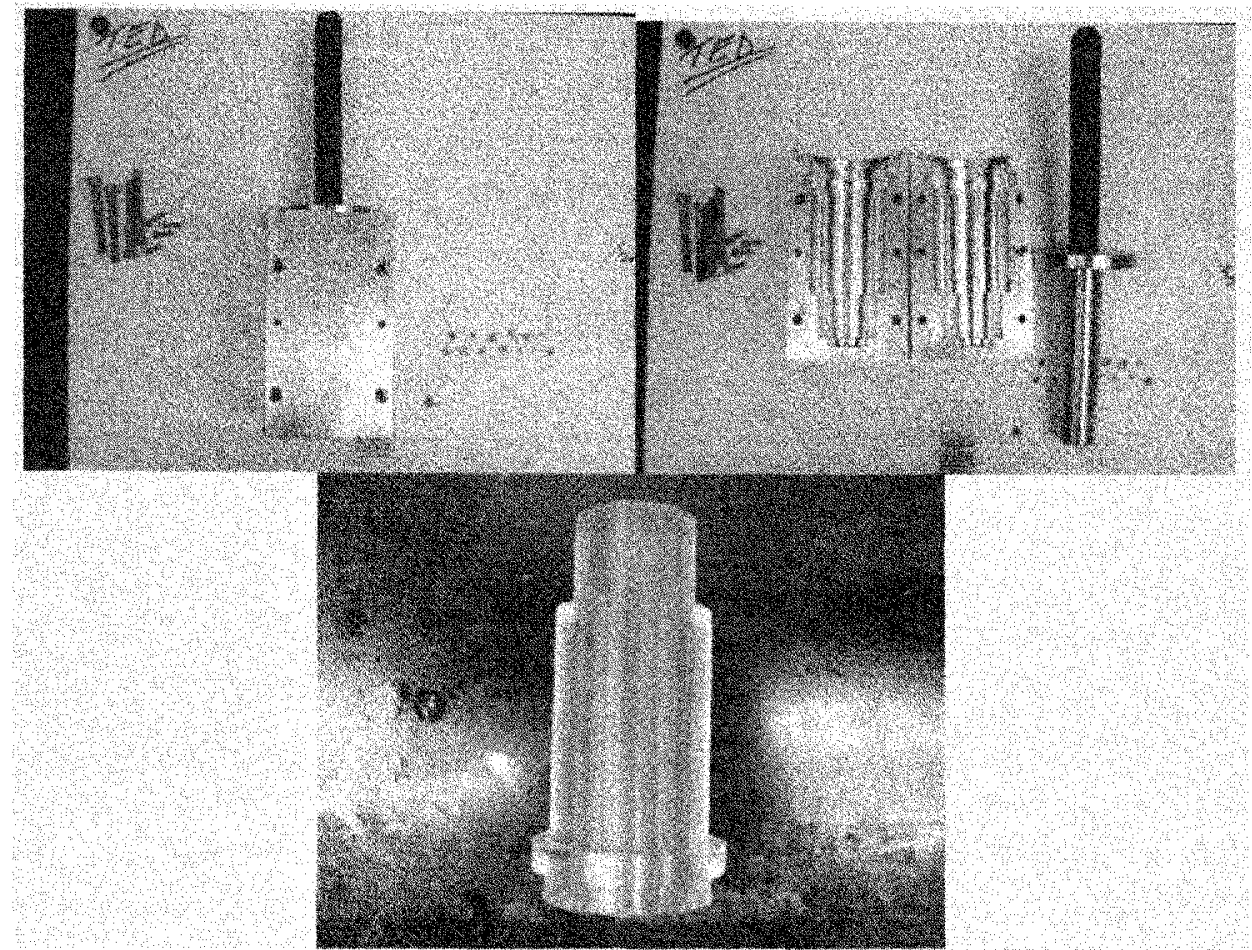

Figure 31: Second generation silicone aorta (SA 2.0) and clam-shell mold.

Initially, compliance of the SA was ignored. The concern instead was structural rigidity such that the SA would form a water tight seal and bypass the compliance chamber during rigorous operation of the LHS. When the proof of concept had been established, compliance was designed into the SA molding process. To that end, spacers were designed for insertion into the mold. This allowed variation of the wall thickness of the SA (figure 32). Moreover, compliance was manipulated by altering the ratio of 
curing agent to elastomer. A larger ratio, i.e. less curing agent, resulted in a more elastic silicone rubber product.

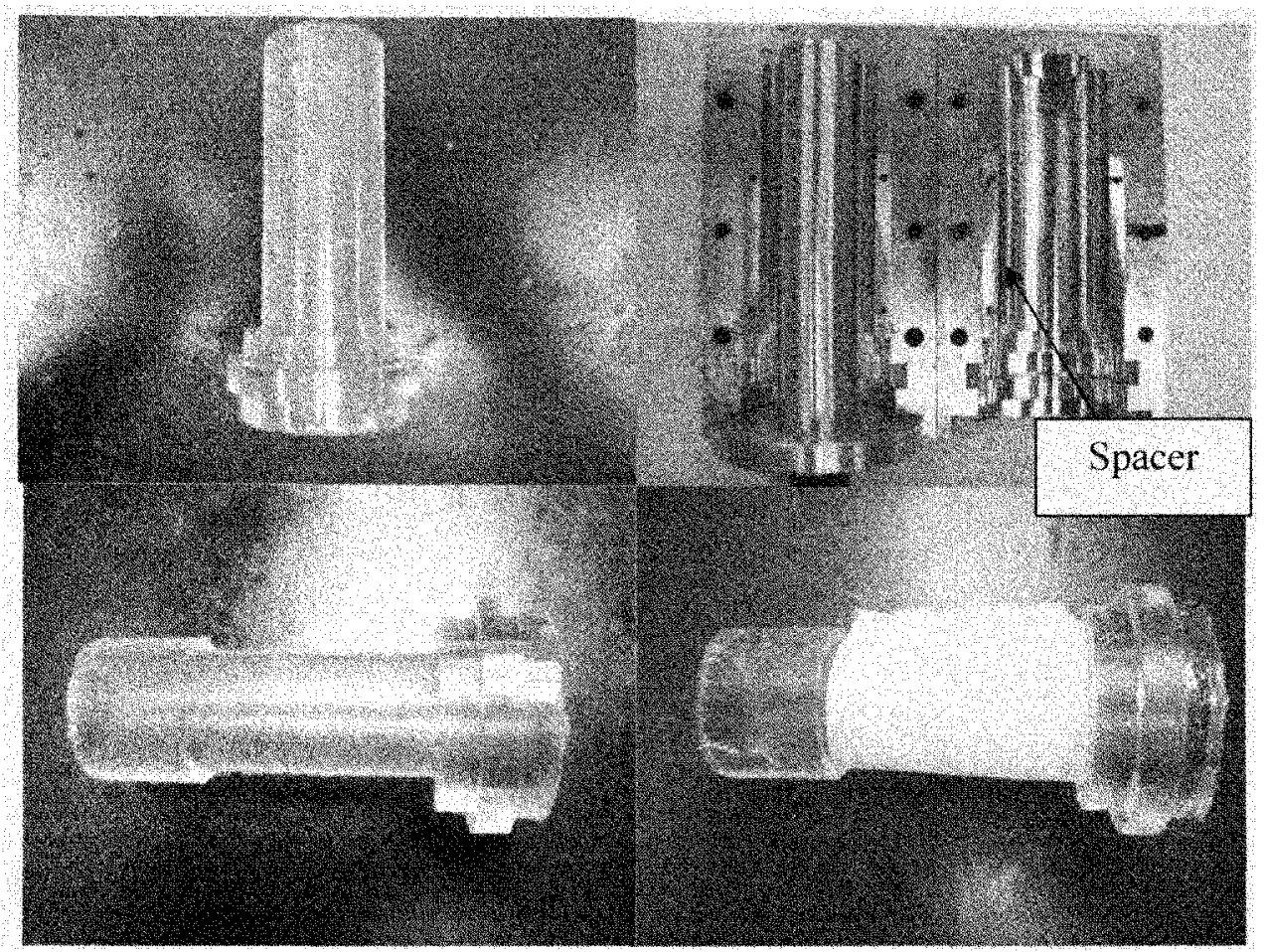

Figure 32: The silicone aorta clam-shell mold with spacer insert for wall thickness modification is shown with SA 2.1 (top left), SA series style 2.2 (bottom left), and SA 2.5 (bottom right). SA 2.5 was wrapped with polyester fabric in order to limit the amount of stretch during testing inside the LHS.

8.7. VERIFICATION TESTING

8.7.1. IN VITRO MODEL

In vitro model verification was performed via Matlab Simulink modeling of a two-element Windkessel model (figure 34). The model used an iterative nonlinear least squares method fit a curve to the pressure waveform data recorded during testing and to calculate the systemic resistance and compliance values for each test run. A good curve 
fit to the data indicated that the Windkessel model was accurately predicting the systemic resistance and compliance values (figure 33). A normal distribution of the residuals centered around zero was an indicator of the goodness of fit.

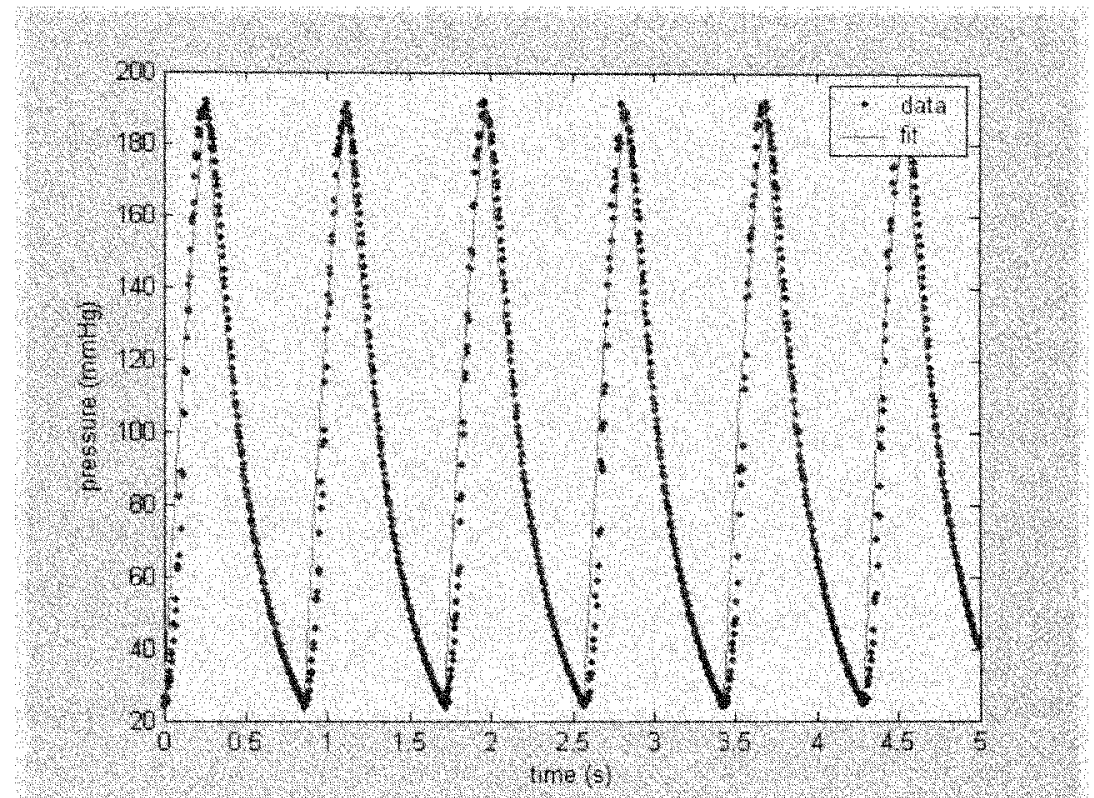

Figure 33: A sample of the Matlab Windkessel nonlinear least squares curve fit to the aortic pressure waveform data collected during a PHV LHS test.

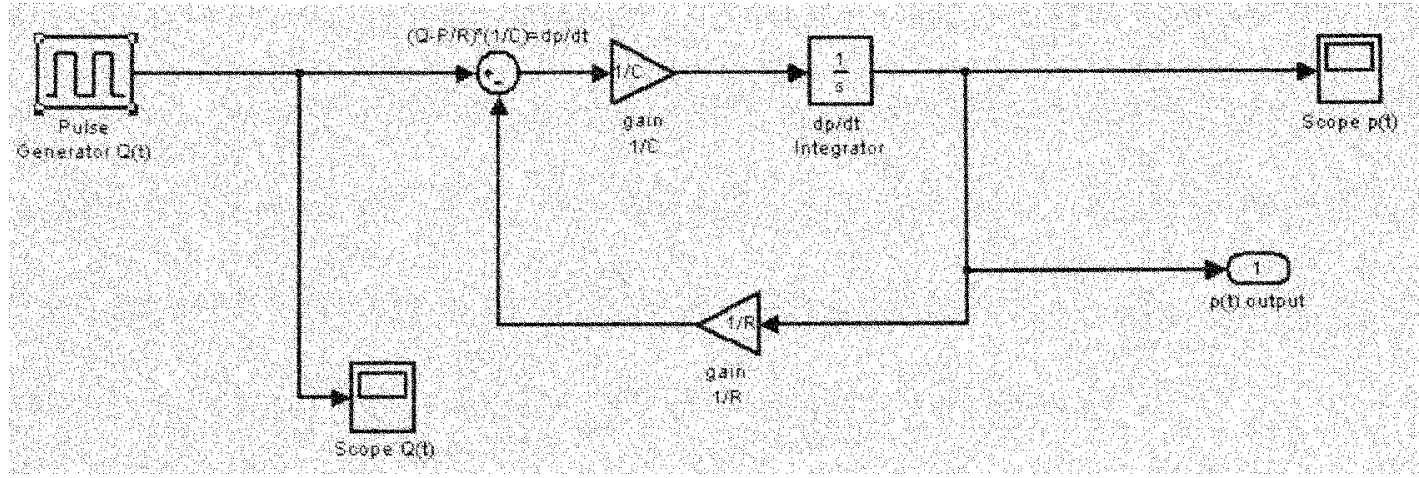

Figure 34: The Matlab Simulink two-element Windkessel model used to numerically solve the differential equation and calculate the coefficients $\mathrm{R}$ and $\mathrm{C}$. 
8.7.2. FIXATION

PHV fixation testing was performed using a method similar to that described in a 2007 paper by Zhou et al., wherein they tested the displacement force of aortic stent grafts by deploying the grafts into animal aortic segments and pulling the grafts axially. Their results showed initial displacement forces between 3 and $33 \mathrm{~N}$ depending on the type of graft and the magnitude of over-sizing (5-20\%). [63] Our tests were conducted by anchoring SA 2.1 to the mobile top anchor of the Bose ELF 3200 via \#1 Ethibond suture and tethering the PHV, deployed inside the SA, to the bottom fixed anchor of the system (figure 35). The suture attached to the PHV was separated and held parallel to the wall of the tube via an aluminum dise so that no bending moments would be applied to the PHV during the test. It was believed that a bending moment applied to the tethered end of the PHV could have caused it to prematurely loosen and slip. The Bose system was set for 6 $\mathrm{mm}$ of displacement, but the value of interest was the force required to cause slippage of the PHV. Therefore, the displacement was not considered critical, i.e. the SA exhibited some elastic deformation prior to PHV slippage during the tests, but this was ignored in terms of potentially confounding variables. It was assumed that the natural aorta would also exhibit elastic deformation both the axial and radial directions in vivo during the cardiac cycle. Moreover, the SA and the PHV were both wetted with the blood analog solution used in the LHS. This was done in order to reduce surface contact friction between the PHV and the inner surface of the SA and to improve the realism of the test. The force required to make the PHV slip was called the fixation force. 


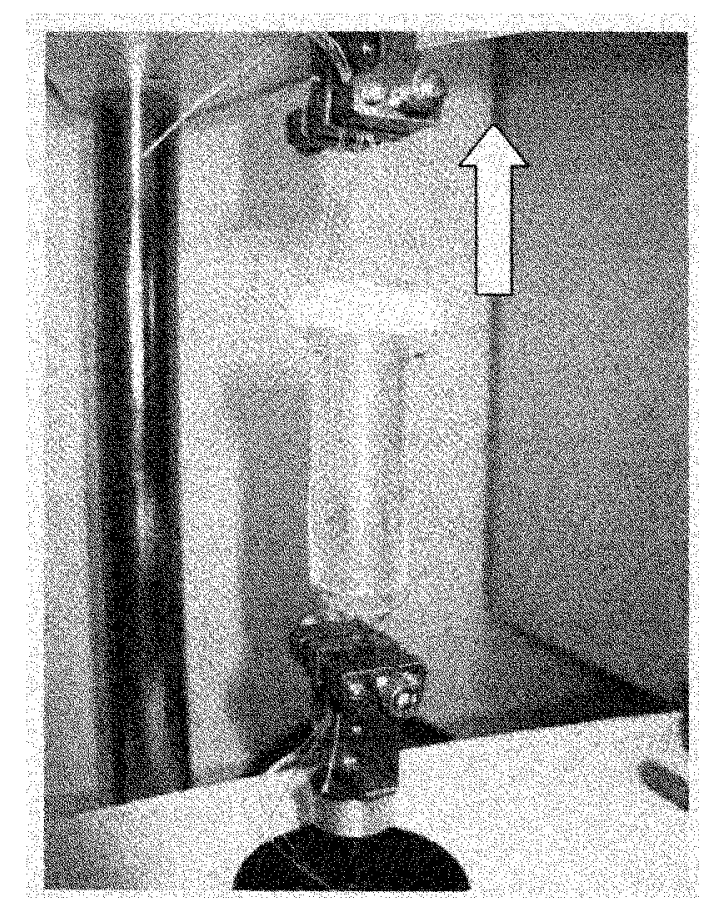

Figure 35: Fixation force testing apparatus. Sapien PHV shown inside wetted silicone aorta 2.1 suspended with \#1 Ethibond sutures. Notice the aluminum disc at the base of the apparatus separating the three sutures tethered to the PHV.

\subsubsection{COMPRESSION}

Each PHV prototype was placed inside a custom made apparatus in order to measure radial compression and axial slippage forces (figures 36 and 37). The radial compression tests were performed using a Teflon sheet that was cut into a shape that would allow the Bose ELF 3200 to convert linear motion into rotational motion, i.e. as the machine increased the linear distance between its two anchors; the Teflon sheet loop would decrease in diameter. Therefore, the force measured was taken to be the hoop force of the PHV. The hoop force was converted to radial force using the relationship shown in equation 32.

Equation 32: Radial Force: $\mathrm{F}_{\mathrm{r}}=\mathrm{F}_{\theta} 2 \pi$ 
Teflon was chosen so that the PHV would be able slip with minimal friction as the diameter of the loop decreased. The thickness $(0.015 \mathrm{inch})$ of the Teflon sheet was chosen qualitatively by testing the balance between tear resistance and stiffness. The starting diameter of the Teflon sheet loop was set to $21 \mathrm{~mm}$ at the beginning of each test using a stainless steel cylinder of the same diameter. The maximum linear displacement of the Bose ELF $3200,12 \mathrm{~mm}$, was executed during each test. The result was an $18 \%$ decrease in diameter for each test. By comparison normal pulse pressure diameter change of the aortic root in a young healthy adult human is in the vicinity of $10 \%$.

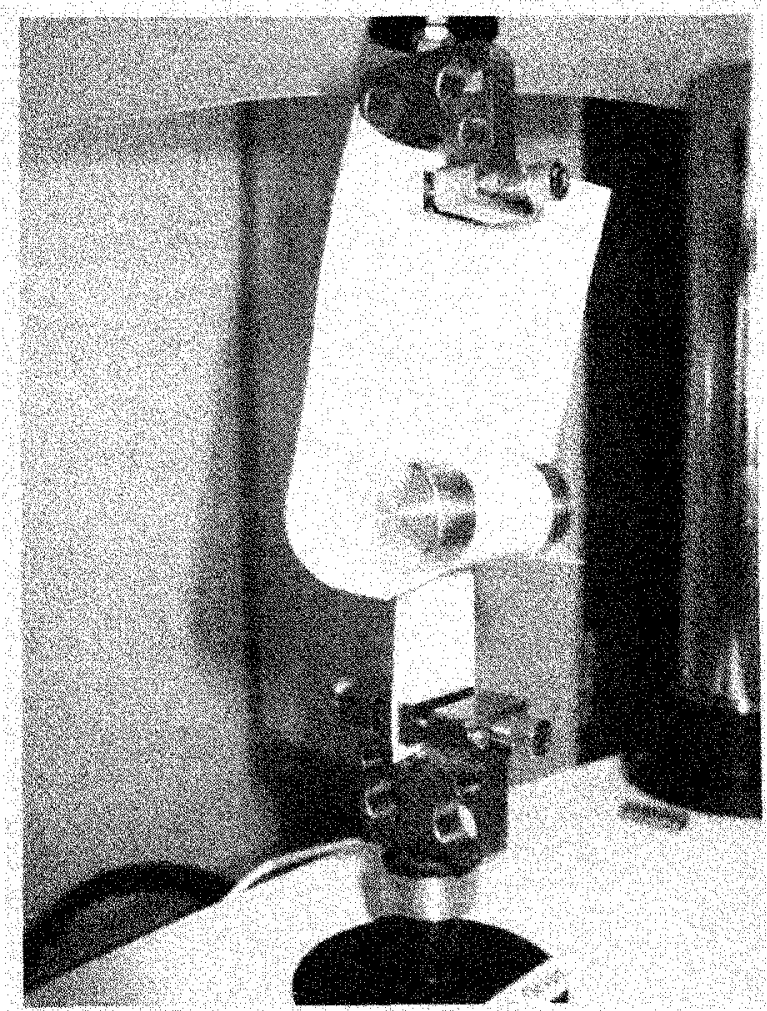

Figure 36: PHV compression testing set-up. A Teflon sheet was used to compress each PHV. The starting diameter of the compression test was set to $21 \mathrm{~mm}$ by using a stainless steel cylinder with a diameter of $21 \mathrm{~mm}$. 


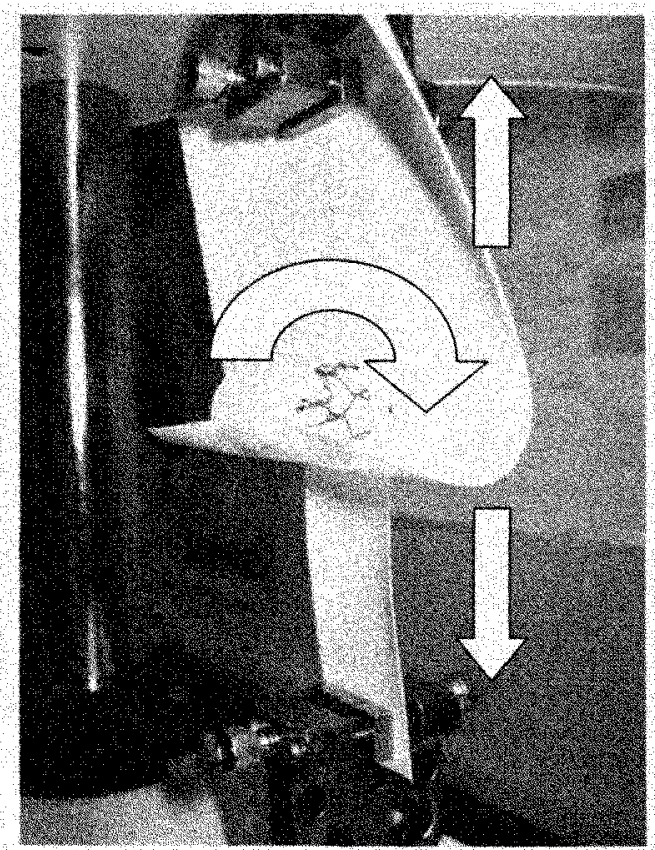

Figure 37: PHV shown inside the compression testing apparatus. Linear force was converted to hoop force. The measured force was taken to be the hoop force for each PHV.

\subsubsection{HYDRODYNAMICS}

Hydrodynamic tests have been performed using the Vivitro Systems, Inc. Superdup'r Left Heart Simulator (figure 38). Twelve test runs per prototype were attempted during each test. Of the twelve total runs, three runs each were performed at $45,70,100$, and $120 \mathrm{bpm}$, at target mean flow rates of 2.3 to $11.4 \mathrm{~L} / \mathrm{min}$ respectively in accordance with the FIU Hydrodynamics Testing Protocol written by Siobhain Gallocher and included in the appendix. LHS test measurements were recorded using a PC running AcqKnowledge 3.2.3 software acquiring data from a Biopac Systems, Inc. MP100A connected to two Millar Instruments Mikro-Tip Catheter pressure transducers inserted into the LHS ventricle and aorta respectively, and a Carolina Medical Square-Wave Electromagnetic Flow Sensor positioned at the based of the aortic outflow tract in the LHS. 


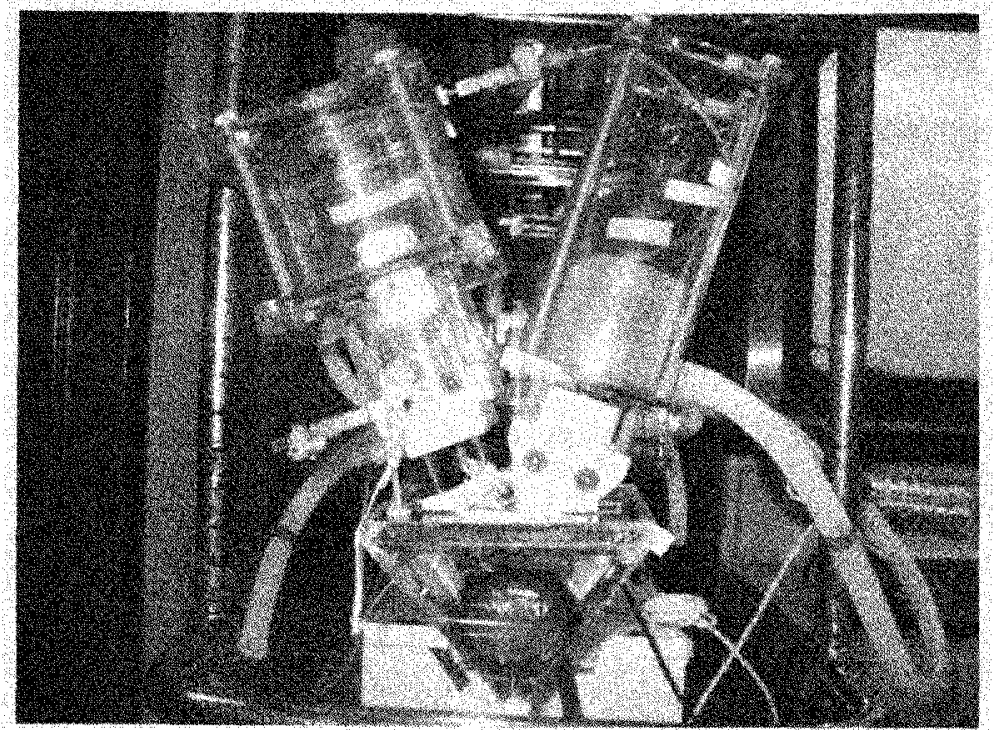

Figure 38: Vivitro Systems, Inc. Superdup'r Left Heart Simulator.

The heart rate was set using a Vivitro Systems, Inc. Tri-Pack TP 2001 Square Wave Generator. The flow was generated using a Vivitro Systems, Inc. Superpump SPA3891 Servo Power Amplifier connected to a Superpump Piston-in-Cylinder System. A blood analog solution composed of $35 \%$ glycerin and $65 \%$ deionized water by volume with 9 grams of sodium chloride per liter was used during LHS testing. The resulting solution had a density of $1.133 \mathrm{~g} / \mathrm{ml}$ and a viscosity of $3.2 \mathrm{cP}$, which was similar to whole blood. Characteristic waveforms produced by the system are shown in chart 1 .

A mock aortic flow loop was designed to redirect flow through the LHS into the loop so that dynamic delivery and deployment testing of the PHV could be performed, i.e. the LHS was operating and the delivery catheter was threaded into the LHS and positioned for deployment of the PHV into the SA inside the LHS.

The compliance of the silicone aorta designed for PHV testing inside the LHS was measured on the bench-top using a Labtron Accumax Blood Pressure Cuff and a Standby Baumanometer (figure 39). Diameter and pressure changes were measured 
using digital calipers with $0.01 \mathrm{~mm}$ accuracy and compliance was calculated using equation 6. Three measurements of the diameter of the SA were taken at a set pressure and then the average of those values was used to calculate compliance.

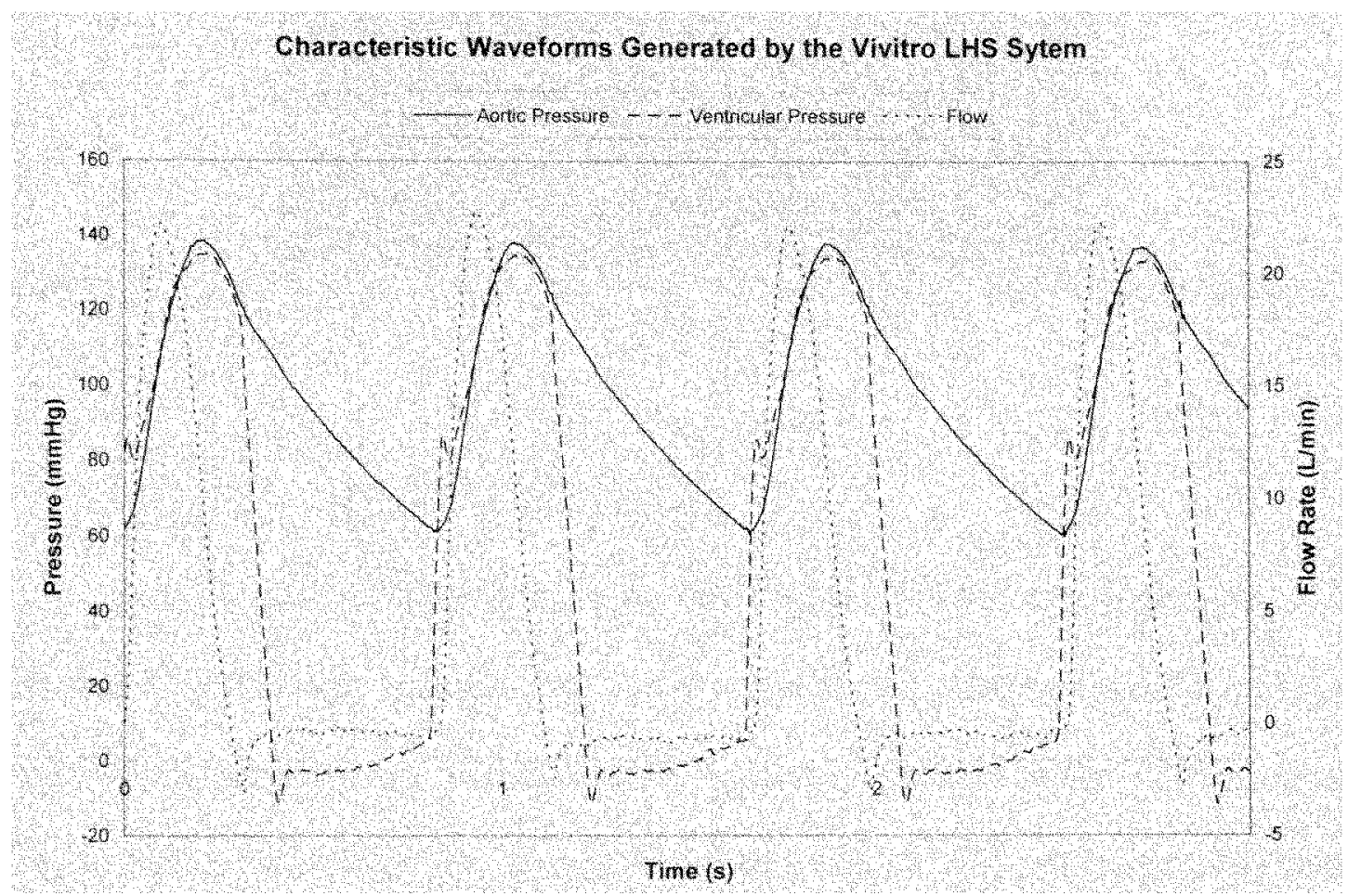

Chart 1: Waveforms generated by the Vivitro LHS with its original settings and a $25 \mathrm{~mm}$ St. Jude bileaflet mechanical valve in the aortic position.

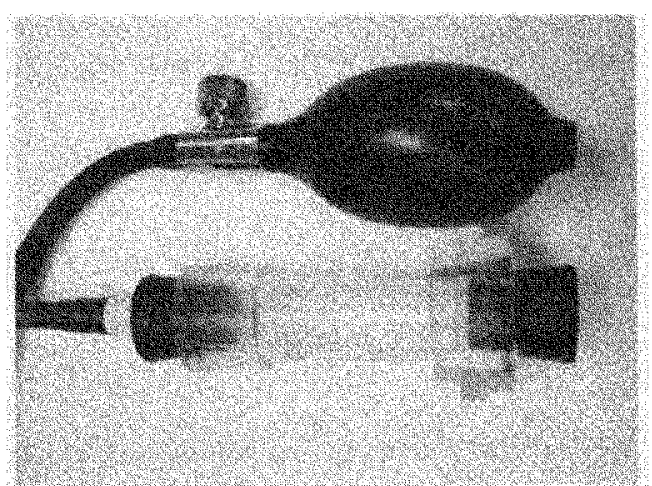

Figure 39: Silicone aorta compliance testing apparatus. 


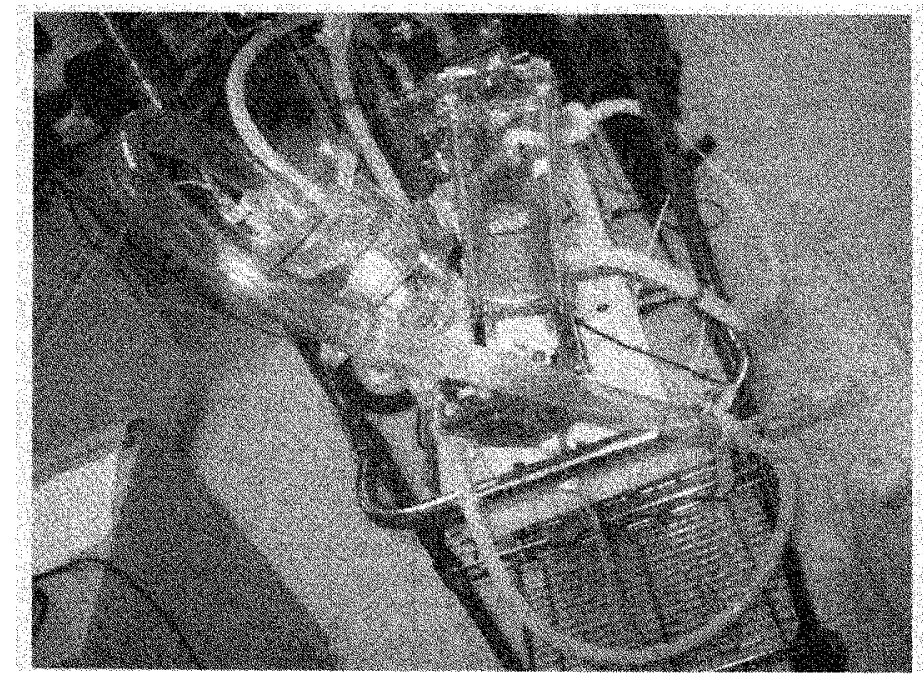

Figure 40: LHS with mock aortic flow loop for dynamic PHV delivery and deployment testing.

\subsubsection{DELIVERY SYSTEM}

Lutter et al. published a paper in 2006 detailing the in vitro testing of a few different PHV delivery systems. [34] For in vitro testing, they constructed a silicone model of the heart and the venous vascular system to simulate antegrade deployment. They measured the devices' physical characteristics, e.g. ID, OD, total length, and usable length, and they created a qualitative scoring method for grading the devices' flexibility or ductility, stiffness, transmission force, handling, and durability. They also measured the device placement time, the stent deployment time, the device retrieval time, the number of tests producing material fatigue, the radius of curvature, and the radial force. Similarly, our delivery system was tested on the bench-top via deployment of the PHV into a one inch diameter polycarbonate tube shaped like an aorta simulating retrograde deployment (figure 41). The test was performed by inserting the catheter into the "aorta", retracting the sheath, deploying the PHV and removal of the delivery catheter from the 
tube. Deflection of the catheter tip was tested by manipulating the steering rod in the hand piece (figure 42). However, the rigorous test methods employed by Lutter et al. were not duplicated. Instead, a purely qualitative assessment of the system was performed, aside from the physical dimensions being recorded, due to time and budget constraints, i.e. the catheter tubing was not ideal for such rigorous testing.

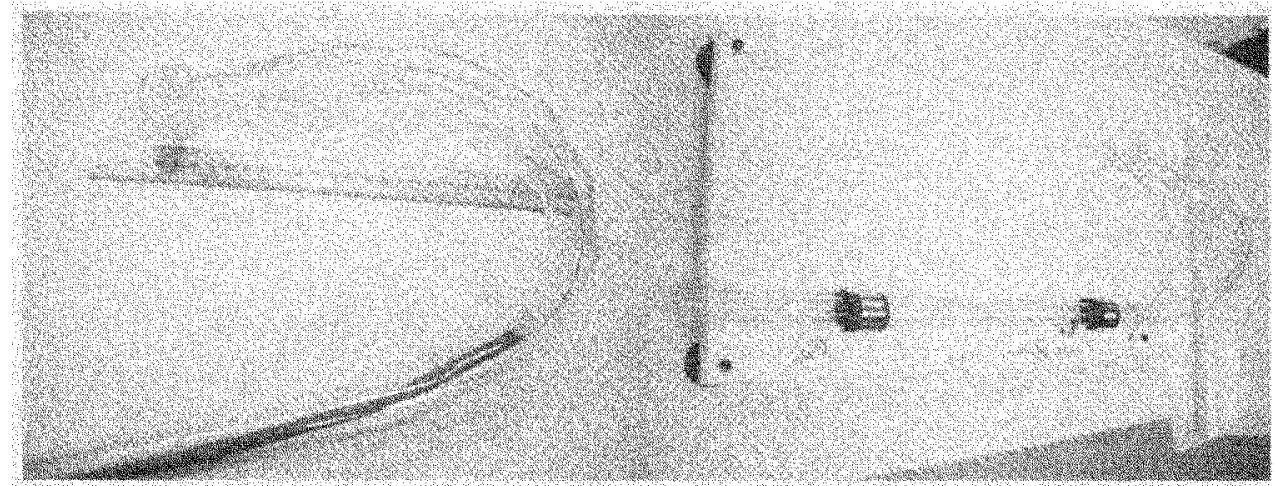

Figure 41: Polycarbonate tube used to test PHV delivery system. A silicone aorta was attached to the end as a receptacle for the PHV.

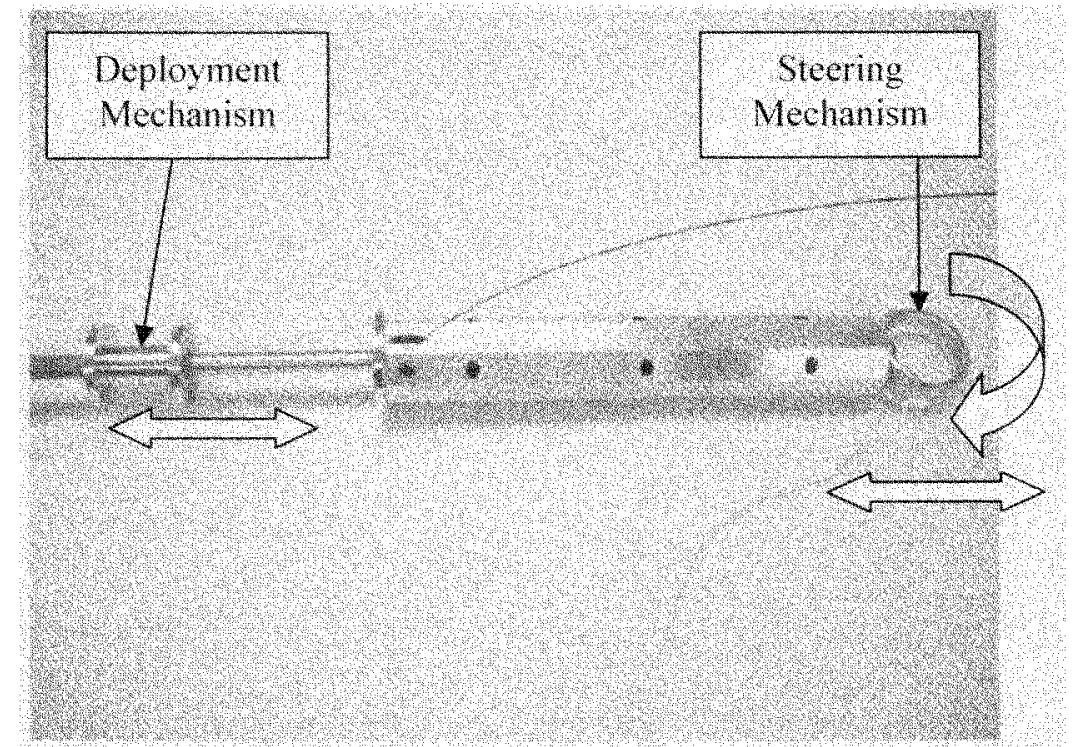

Figure 42: Delivery system hand piece. Guide wire is shown protruding from the slot. The steering wire is shown attached to the ring. 
The data set from each LHS test has been processed via a custom Matlab 6 program designed to calculate the aforementioned hemodynamic parameters, which is included in the appendix. The resulting data was statistically analyzed using SPSS 13.0 software. One-way ANOVA and Tukey's post hoc tests were performed for each parameter of interest in order to compare the difference between means of each prototype.

\section{RESULTS}

\subsection{BASELINE: ST.JUDE VALVE}

The LHS was designed for the testing of surgically implanted heart valves. Therefore, a baseline test of the LHS with a $25 \mathrm{~mm}$ diameter St. Jude bileaflet mechanical valve was performed for comparison to PHV performance. These results were considered the "gold standard" for which the PHV development in this research was striving. The tests showed a mean valvular pressure gradient of $4.3163 \mathrm{mmHg}$ and a mean regurgitation of $5.7851 \%$.

\subsection{FJA-MCB}

\subsubsection{ORIGINAL}

Work began with the replication of the FJA hydrodynamic tests of his MCB PHV (figure 43). The valve was clearly worse for the wear at the time of the test. It is shown partially disassembled as a result of later investigations into construction methods. 


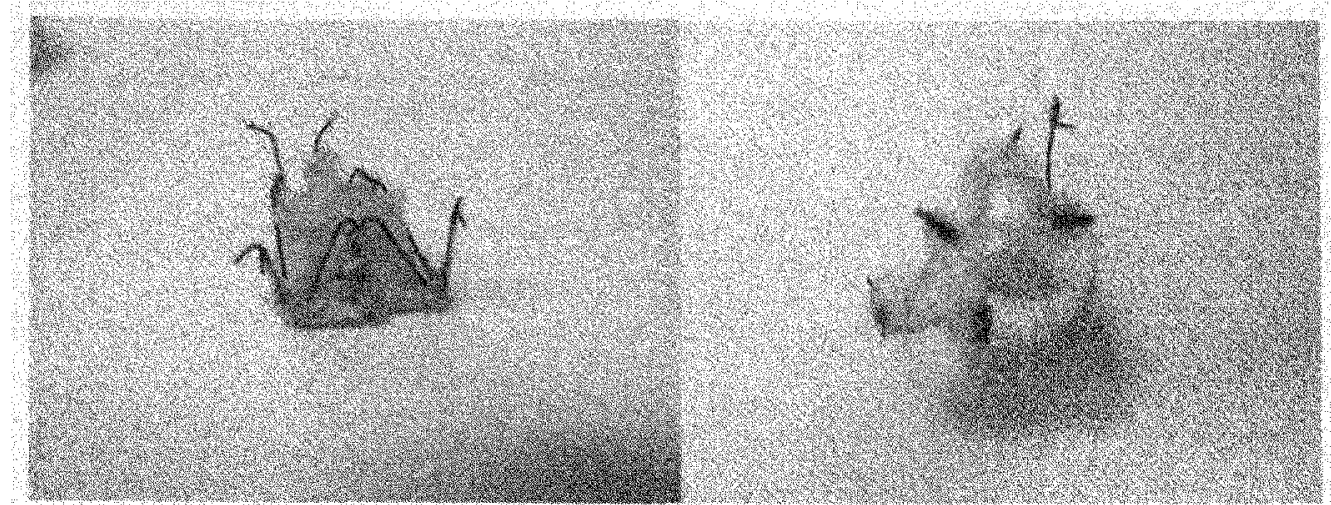

Figure 43: Original FJA-MCB prototype. Wear and tear is evident. Notice the relaxed triangular shape.

This work began by obtaining porcine hearts from a local slaughter house (Cabrera's). The aortic root was dissected from the heart while leaving the native aortic valve intact. SIBS-polyester composite sheets were sutured to each end of the vessel using silk suture as shown in figure 44 . The vessel was mounted onto the FJA porcine aorta fixture shown in figure 45. The assembly was placed inside the LHS as shown in figure 46.

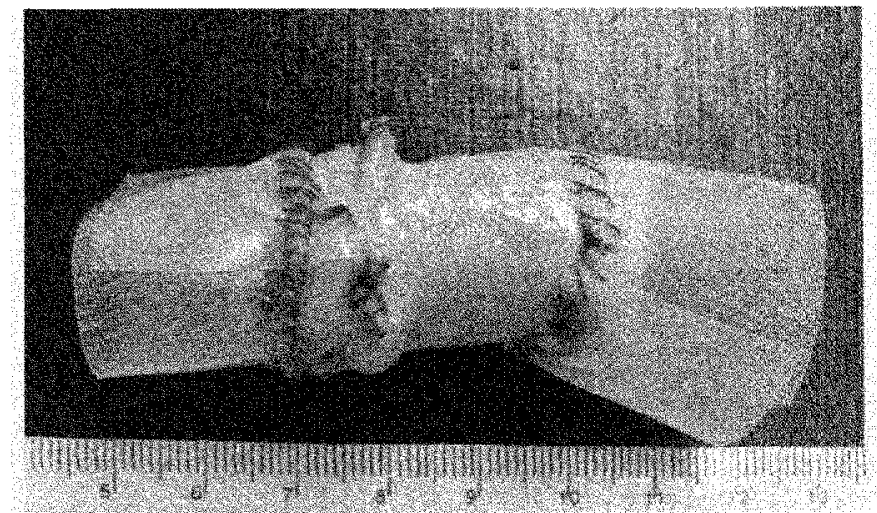

Figure 44: Photo from FJA Thesis showing polymer coated polyester sheet attachment to a dissected porcine aortic root. 


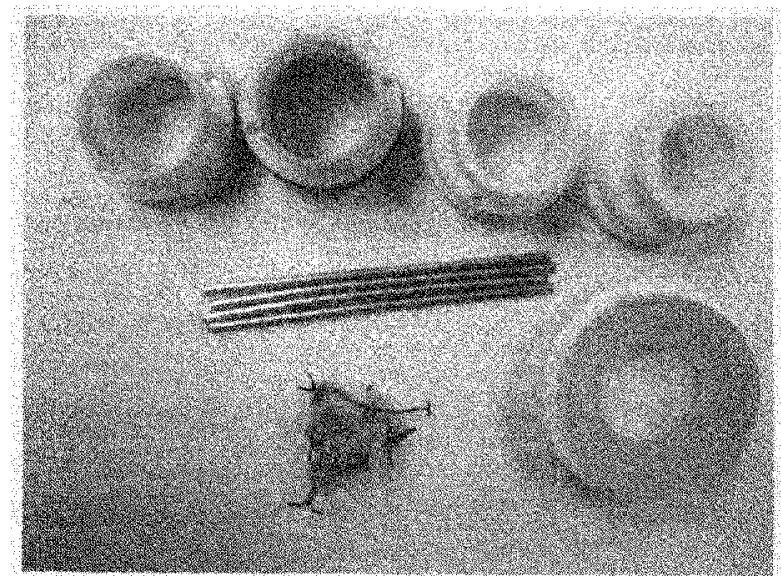

Figure 45: FJA porcine aorta fixture and MCB valve shown with various sized outlets.

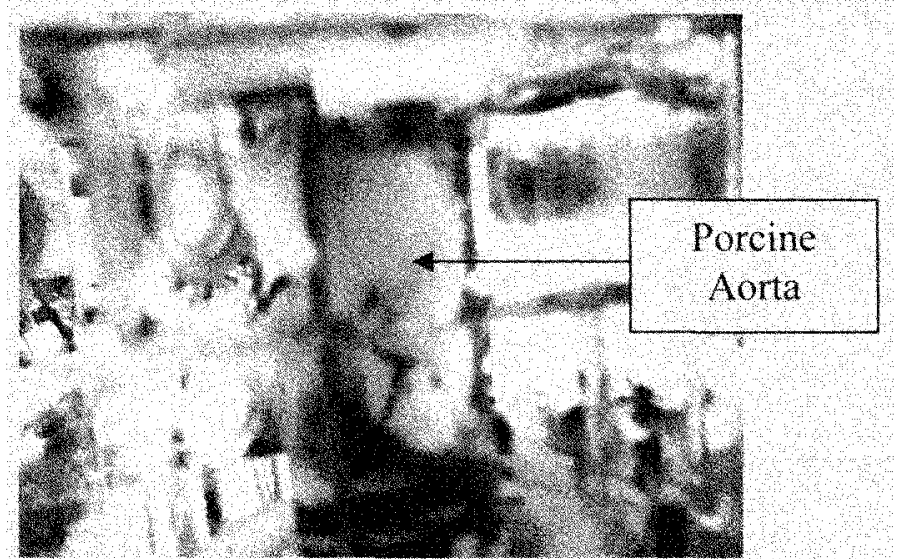

Figure 46: Porcine aorta inside the LHS during testing.

Initially, the porcine aortic segment and natural valve were tested alone. Physiological flow rates and pressures could not be achieved due to observed collapse of the aorta during systole and apparent looseness of the vessel attachment to the FJA fixture. Therefore, no data were recorded.

Later, the vessel was adjusted for fit, tension, and leakage. The FJA-MCB valve was then inserted into the native porcine valve, and the assembly was placed inside the LHS for a second test. Again, the data from the experiment was not recorded because physiologic flow could not be achieved, i.e. $\mathrm{MAP}=90 \mathrm{mmHg}$ and $\mathrm{CO}=5 \mathrm{~L} / \mathrm{min}$. As 
before, vessel collapse during systole and flow leakage at attachment sites was observed. The collapsing of the vessel distorted ventricular pressure readings by causing the pressure to spike to maximum at each cardiac cycle. Another attempt was made to tighten the fit of the aortic root, but improvement in the hemodynamics was minimal. The test was repeated using other porcine aortic roots, but the same results were obtained. Furthermore, accurate deployment of the FJA-MCB valve was hampered by difficult visualization and hook impingement on the inner surface of the delivery sheath. The PHV itself exhibited very little movement, and therefore was insufficient. In all tests the maximum porcine aorta diameter obtained was $17 \mathrm{~mm}$. The FJA-MCB valve was designed for a $19 \mathrm{~mm}$ vessel. It was determined that the use of a porcine aorta for PHV testing was laborious and difficult to control, and therefore would result in inconsistent test conditions and bias the test results.

The experience lead to the realization that at the FJA-MCB valve had to be rebuilt due to the degraded condition of the tested valve. Additionally, the decision was made to design an alternative test conduit to replace the porcine aorta.

\subsubsection{REBUILD}

The results obtained from the test of the original FJA-MCB valve prompted the fabrication of a new FJA-MCB valve prototype for testing. The process of doing so facilitated learning of the FJA PHV manufacturing method. A new FJA-MCB valve was constructed based upon information in the FJA thesis and physical inspection of the original FJA-MCB valve in possession. A detailed description of the FJA-MCB PHV construction was written by the author and is included in the appendix. The original FJA 
Nitinol annealing plates were used to shape the 0.019 inch diameter Nitinol wire into the proper shape. The shaping process required two heating cycles; the first step set the primary wire turns and the second step set the bends that would become the hooks. Some improvisation was required in the process due to missing components of the FJA assembly system. The initial wire bending pattern was found by iteratively bending copper wire in various shapes, as shown in figure 47.

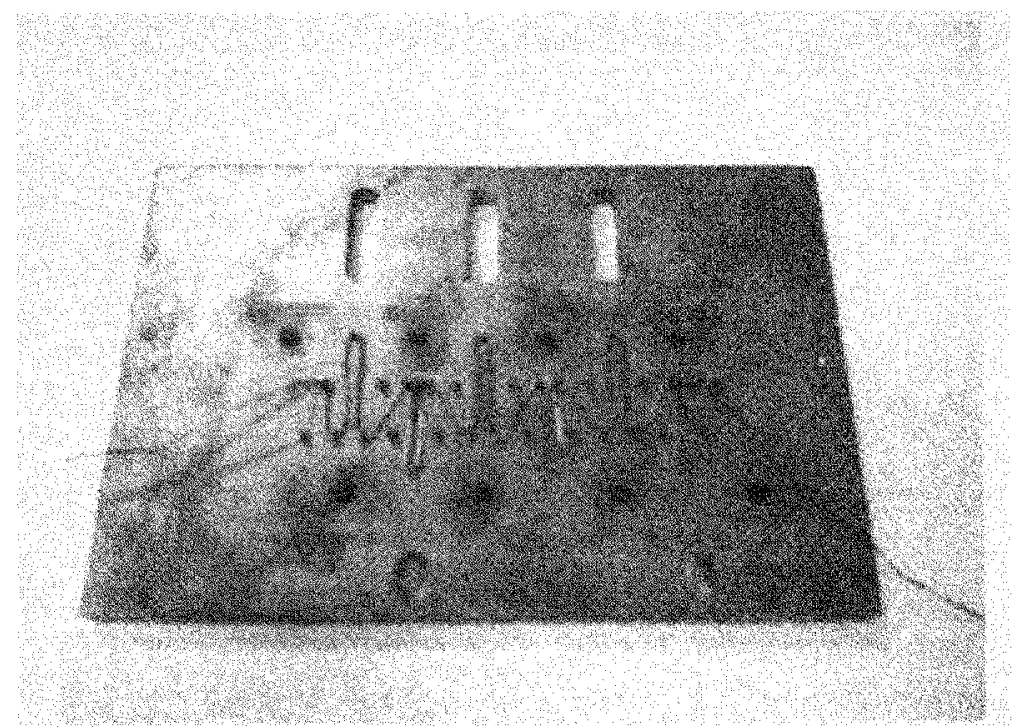

Figure 47: Process of determining wire forming pattern for FJA-MCB using the FJA annealing plates and copper wire.

Each stent segment was joined using 0.040 inch ID thin-walled stainless steel hypo-tubing. However, FJA specified the use of 0.032 inch ID hypo tubing, but after several unsuccessful attempts to pass two 0.019 inch $(D=0.038$ inch) wire ends through a segment of the tubing it was abandoned for the larger tubing. The leaflet material was fabricated using the FJA polyester fabric remaining in the CVEC and the SIBS polymer. The result was a material with a thickness of about 250 microns. The leaflet material was 
cut according to the FJA method for creating "central coaptation" leaflets. [69] The three leaflets were joined and mounted onto the stent using Ethibond sutures. The resulting FJA-MCB PHV is shown in figure 48.

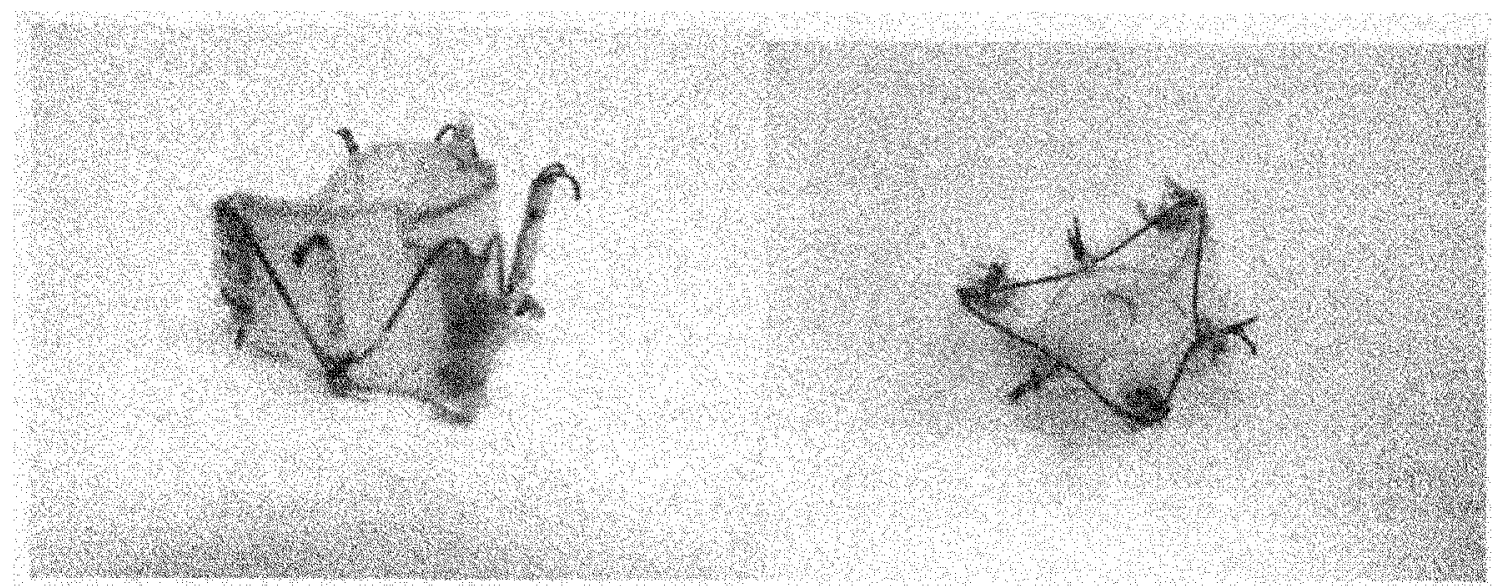

Figure 48: New FJA-MCB valve by Claiborne. Notice the characteristic relaxed triangular shape.

The new FJA-MCB valve was mounted inside the first generation silicone tube (SA 1.0) and dilated using a Bard $12 \mathrm{~F}$ urinary catheter with a $5 \mathrm{ml}$ balloon as shown in figure 49. The urinary catheter balloon was latex and therefore could not exert high radial forces akin to polyurethane vascular balloon catheters, but it was sufficient for this experiment. Its purpose was dilatation of the PHV and embedment of the flow retention hooks into the silicone aorta wall. As with the former FJA-MCB physiological values could not be obtained, but the valve performed well enough to record some data. However, the valve showed proper trends in performance as shown in charts 2 and 3. The valve was tested at $70 \mathrm{bpm}$ for nine runs. The mean cardiac output was $5 \mathrm{~L} / \mathrm{min}$, the mean arterial pressure was $34.5 \mathrm{mmHg}$, the mean pressure drop across the valve was 12.4 $\mathrm{mmHg}$, and the mean regurgitation was $41 \%$. 


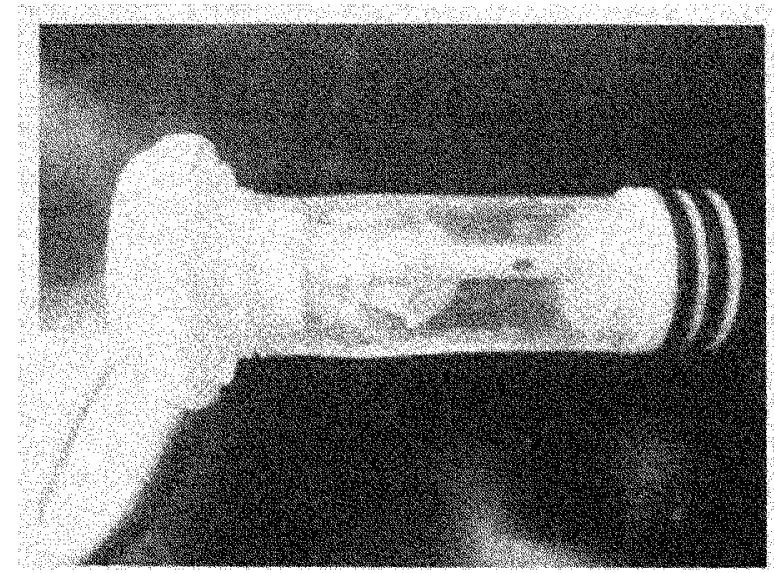

Figure 49: SA 1.0 mounted onto the FJA porcine aorta fixture with new FJA-MCB in situ. A urinary catheter balloon was used to open and fix the PHV inside the SA.

A mean regurgitation of $50 \%$ would be expected if there were no valve in the test conduit. This can be explained by the observations that the valve did not have a good fit inside the SA (figure 50), i.e. it appeared to be somewhat oversized as evidenced by the buckling and overlap of the leaflets in the unload conformation. Moreover, the outer edges of the valve did not lay flush with the wall of the tube even after balloon dilatation. The hooks were observed to be protruding through the tube wall, but some shifting of the valve occurred during testing.

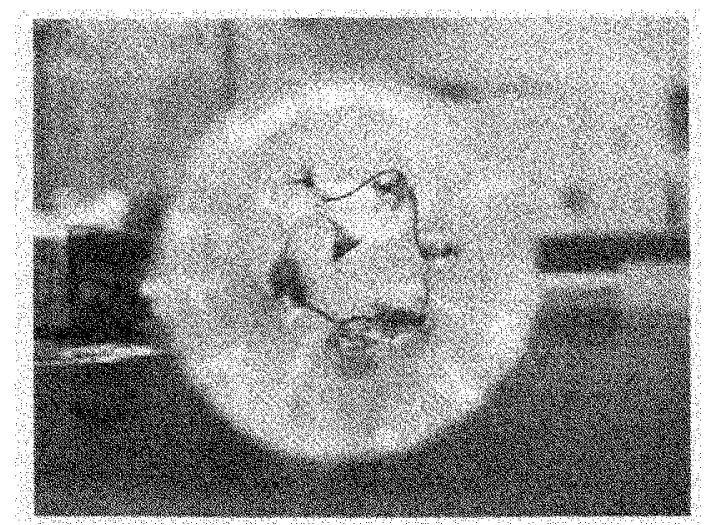

Figure 50: New FJA-MCB valve inside the first generation $19 \mathrm{~mm}$ diameter silicone aorta. Notice the leaflets appear to be oversized. The view is looking proximally toward the ventricle. 


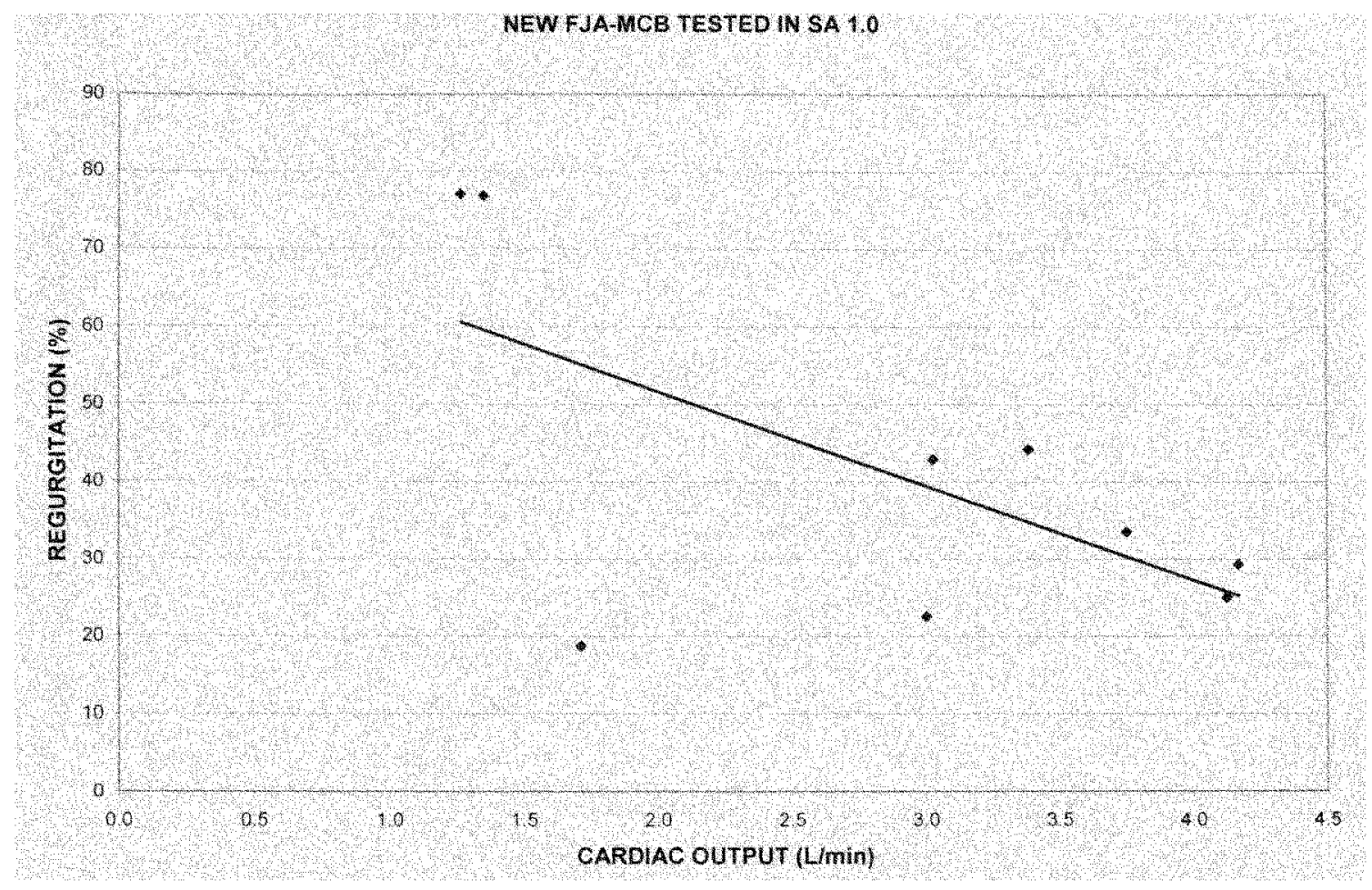

Chart 2: New FJA-MCB valve test in first generation silicone aorta. The trend of regurgitation as a function of cardiac output data shown here is as it should be for a heart valve, but the slope is too steep and the values show a wide variation in distribution. 


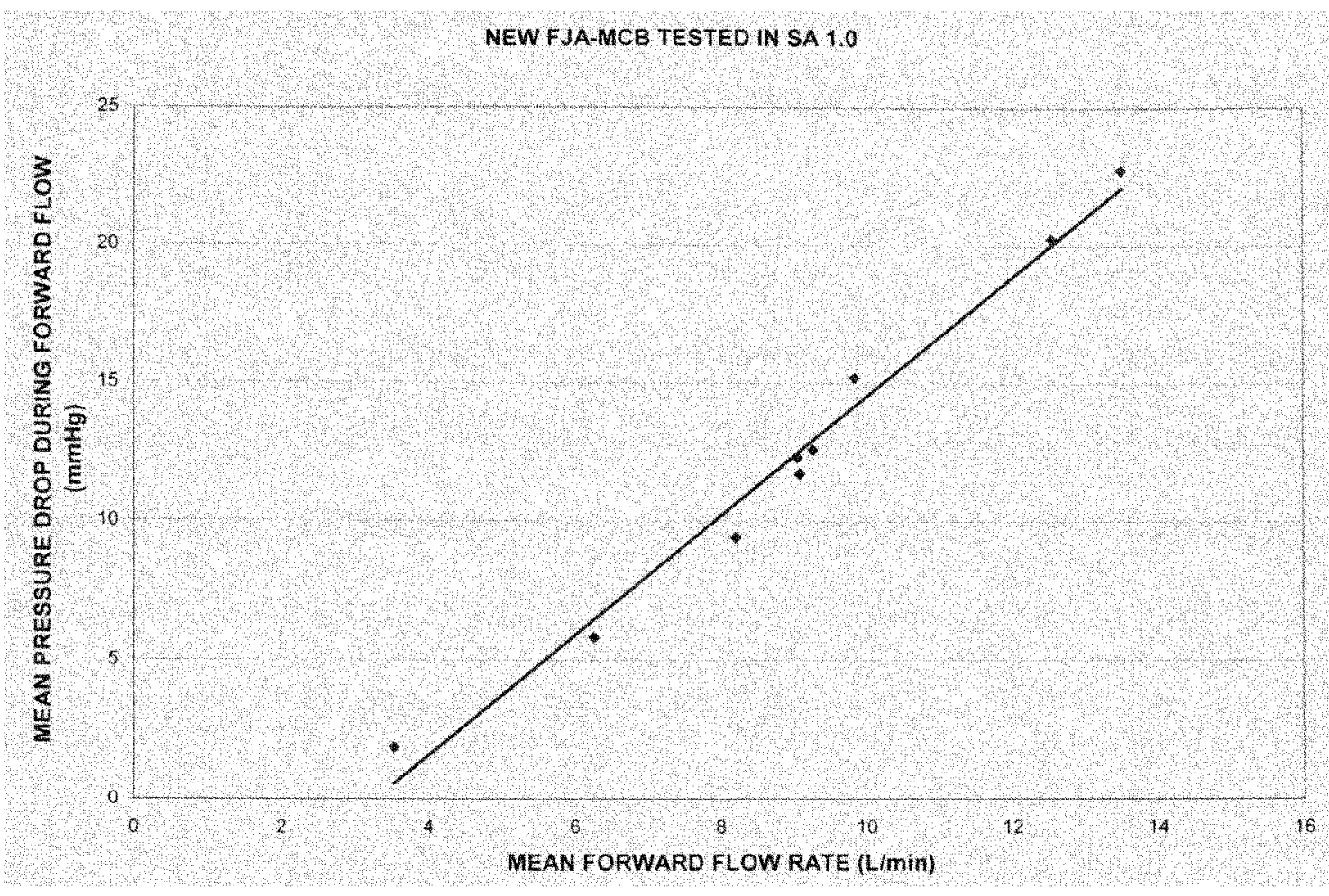

Chart 3: New FJA-MCB valve tested in the first generation silicone aorta. The trend in mean forward flow pressure drop as a function of mean forward flow rate is as it should be for a heart valve but again the slope is too steep. However the grouping of data points shows a good fit to the trend line.

\subsubsection{RETEST: SA 2.0}

It was hypothesized that a portion of the poor performance of the new FJA-MCB valve could be attributed to the poor design of SA 1.0. Therefore, the SA mold was redesigned. The new mold design and resulting SA eliminated the need for the FJA porcine aorta fixture and improved the manufacturability and quality of the silicone aortas. The SA 2.0 was used to retest the new FJA valve and the FIU-Hydrodynamics testing protocol written by graduate student Siobhain Gallocher was followed. This test exposed the valve to much higher flow rates than the initial test with the first generation silicone aorta. During this test the pressure drop across SA 2.0 without a PHV was 
measured. The introduction of smaller diameter flow conduit to the LHS produced an intrinsic pressure gradient. This gradient was assumed to be additive to the PHV induced gradient and was therefore subtracted from all raw PHV pressure drop data points to arrive at an "adjusted" value. This value was called the "tube factor" ( $4.65 \mathrm{mmHg}$ for SA 2.0). Results produced a mean adjusted mean pressure gradient of $37 \mathrm{mmHg}$ and a mean regurgitation of $15.4 \%$, with a mean cardiac output of $6 \mathrm{~L} / \mathrm{min}$ and a mean pressure of about $50 \mathrm{mmHg}$. These results were a slight improvement overall, but a large improvement in terms of regurgitation, with a $62 \%$ improvement. So the fit and sufficiency of the valve improved with the new silicone aorta.

\subsubsection{FJA VALVE SUMMARY}

Once replication of the FJA-MCB PHV valve and tests were complete and the data had been analyzed, an objective assessment of the valve and test methods was executed. The weakness noted in the FJA-MCB PHV design and testing process were (1) a lack of radial force in the stent design, which effectively negated the intended selfexpanding characteristics of Nitinol, (2) poor leaflet function in terms of insufficiency and pressure gradient, (3) the use of hooks for fixation added to the difficulty of deployment of the PHV by impinging on the delivery device inner surface, (4) the design was overly complicated in terms of requiring many pieces and steps to be manufactured, (5) the method of using a porcine aorta for PHV LHS tests introduced variability, increased labor, and produced unreliable results, (6) the FJA test results could not be replicated. The strengths noted were the robust stent annealing and shaping process and the elimination of potential designs for a PHV and testing methods. For these reasons the 
FJA-MCB design and primary test methods were abandoned. The next phase was to use this information to start designing a novel PHV and to refine the testing process.

\subsection{NOVEL PHV PROTOTYPING}

\subsubsection{TC PHV 0}

The first conceived prototype was a simple design that was intended to test manufacturability of PHV's in the lab and to flesh out an initial concept (figure 51). The valve was not hydrodynamically tested.

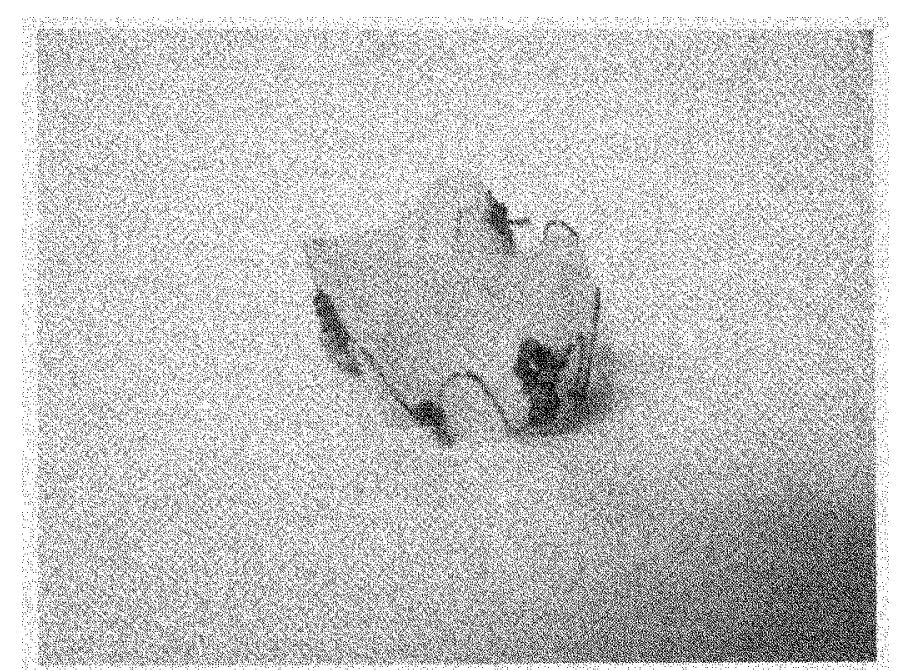

Figure 51: TC PHV 0 concept.

\subsubsection{TC PHV 1}

Design of the TC PHV 1 prototype was based upon all the prior research performed (figure 52). The initial goal was to create a design that could survive LHS testing. For rapid prototyping and to keep costs down, 0.019 inch OD 304 stainless steel wire and the FJA polyester fabric-SIBS composite were utilized. The stent was a simple Z-stent shape with added tear-drop bends that were designed to increased stiffness in 
each turn of the wire. The SIBS-polyester composite leaflet material was formed into a cylinder that was attached to the stent distally at three equally spaced intervals. The valve design was a collapsing cylinder which was meant to mimic the construction and movement of a natural aortic valve. Two stents were formed and joined together at the ends using 0.040 inch ID, thin-walled, 304 stainless steel hypodermic-tubing. Each half of the stent and the leaflet material was joined using 4-0 Ethibond Excel polyester suture.

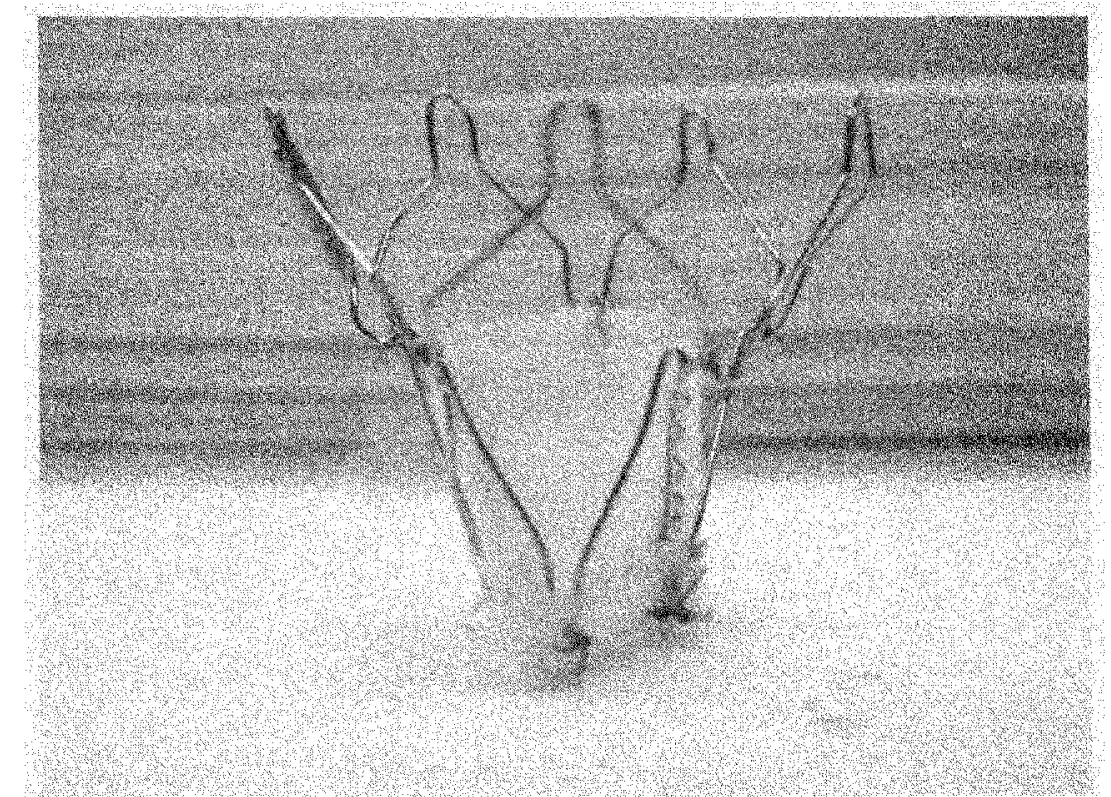

Figure 52: TC PHV 1. The upper bare stent was intended for fixation.

The LHS test of TC PHV 1 was conducted using SA 2.0 and ended abruptly with catastrophic failure of the valve (figure 53). The valve completely prolapsed and the stents dehisced at the sutures. Table 2 shows the data recorded prior to failure. The initial data indicated the presence of a high pressure drop and a large amount of regurgitation. 


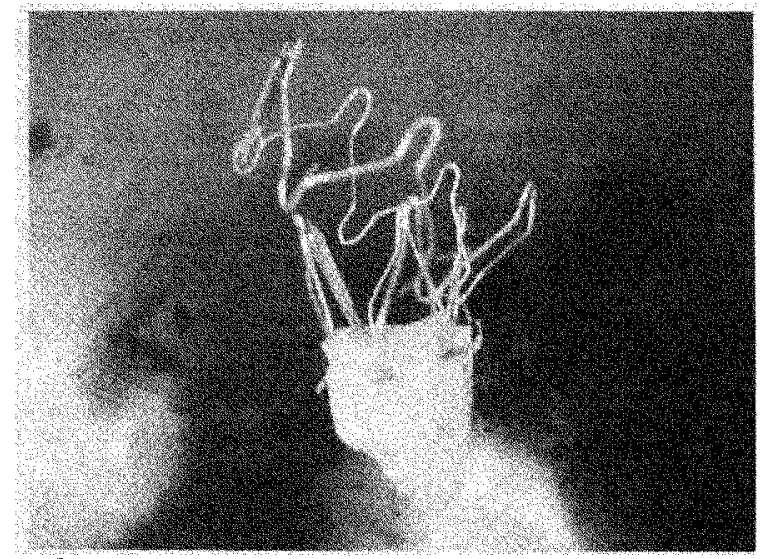

Figure 53: TC PHV 1 after LHS test. The two stents separated and the valve completely prolapsed.

\begin{tabular}{|c|c|c|c|c|c|c|c|c|c|c|}
\hline Scenario & $\begin{array}{c}\mathrm{HR} \\
(\mathrm{bpm})\end{array}$ & $\begin{array}{c}\text { MAP } \\
(\mathrm{mmHg})\end{array}$ & $\begin{array}{c}\text { Positive } \\
\text { Mean } \Delta \mathrm{P} \\
(\mathrm{mmHg})\end{array}$ & $\begin{array}{c}\text { Positive } \\
\text { Max } \\
\text { Pressure } \\
(\mathrm{mmHg})\end{array}$ & $\begin{array}{c}\text { Cardiac } \\
\text { Output } \\
(\text { Limin) }\end{array}$ & $\begin{array}{c}\text { Stroke } \\
\text { Volume } \\
(\mathrm{mL})\end{array}$ & $\begin{array}{c}\text { Mean } \\
\text { Forward } \\
\text { Flow Rate } \\
(\text { L/min) }\end{array}$ & $\begin{array}{c}\text { Leakage } \\
\text { Flow } \\
\text { Volume } \\
(\mathrm{mL})\end{array}$ & $\begin{array}{c}\text { Mean } \Delta P \\
\text { during } \\
\text { forward flow } \\
(\mathrm{mmHg})\end{array}$ & Regurgitation \% \\
\hline TCPHV1 & 70 & 60.3477 & 34.1781 & 116.0407 & 2.1414 & 68.0183 & 11.7659 & 37.4581 & 29.7066 & 55.0706 \\
\hline
\end{tabular}

Table 2: TC PHV 1 test data prior to valve failure.

\subsubsection{TC PHV 2}

The results from TC PHV 1 indicated that the design needed to be much more robust. TC PHV 2 saw the addition of three axial support struts and more sutures all around using the same 0.019 inch SS wire (figure 54).

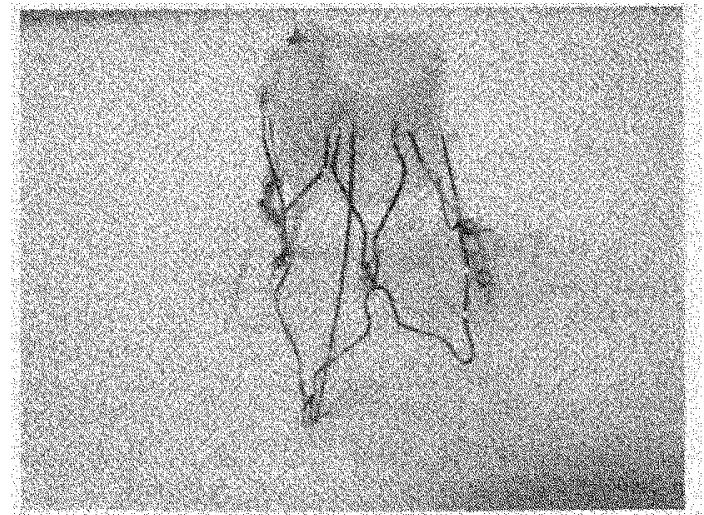

Figure 54: TC PHV 2. Notice the vertical struts and reinforcing sutures. 
The valve was an improvement over TC PHV 1. The adjusted mean pressure drop was $31 \mathrm{mmHg}$ and the mean regurgitation was $21 \%$ as tested in SA 2.0. As intended the design changes strengthened the PHV and it did not have any structural failures. However, the axial support struts were in the flow stream and may have impeded flow. Moreover, it was difficult to manufacture and secure the struts for testing. Therefore, the design of free component struts was abandoned. However, it was noted that some sort of axial stiffness would be required for future designs. Such a feature would prevent sections of the PHV from moving relative to one another. This would be a useful characteristic in deployment of the PHV and presumably in its in vivo operation.

\subsubsection{TC PHV 3}

The next iteration of PHV was completely different. The intent was to fabricate a nitinol frame PHV that did not require annealing for shape forming and one which aggressively addressed regurgitation and perivalvular leakage. The resulting "out-of-thebox" PHV is shown in figure 55.

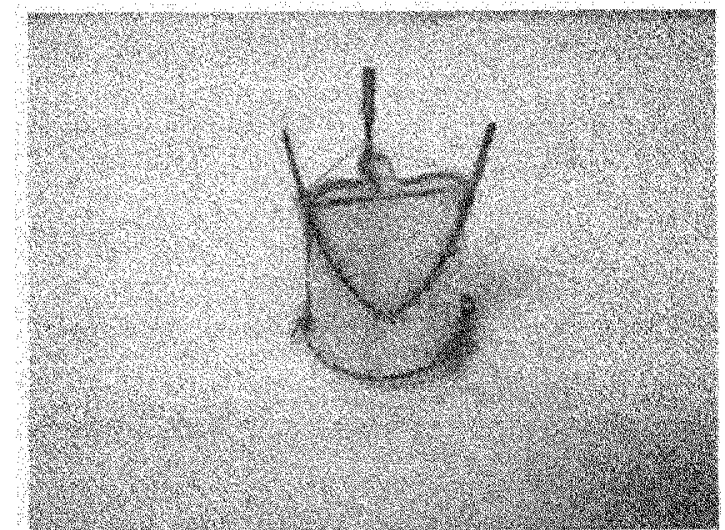

Figure 55: TC PHV 3 with 0.020 " OD SEN wire at the annulus and 0.015 " OD SEN wire supporting the leaflets. 
During the initial test using SA 2.0 some migration was observed, so a stainless steel fixation stent was attached to TC PHV 3 and the LHS test was continued (figure 56). The valve performed well in terms of pressure drop, with an adjusted mean value of $23 \mathrm{mmHg}$, but the design had the opposite effect on regurgitation, with a mean value of 26\%. Due to time and cost constraints, it was determined that the design of TC PHV 1 was the path that would be developed during this research.

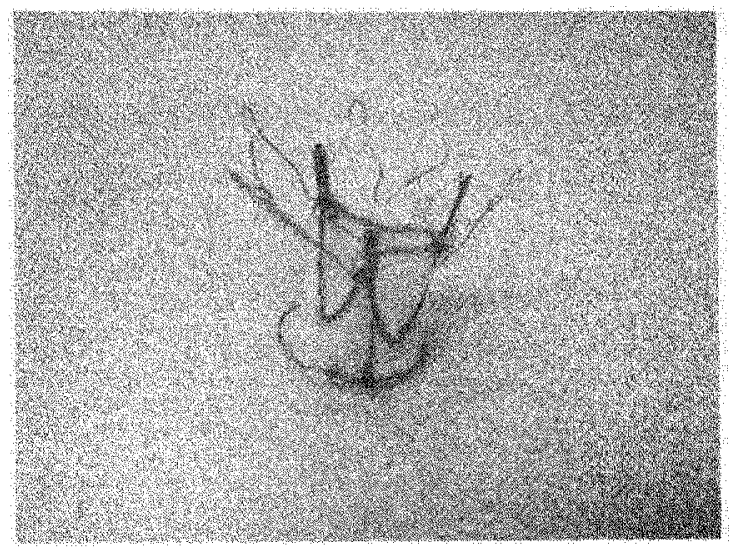

Figure 56: TC PHV 3 with 0.015 " OD SS wire stent.

\subsubsection{TC PHV 4.0}

With TC PHV 4.0, a return the design concept of TC PHV 1 was made. However, the vertical struts of TC PHV 2 were abandoned due to the increase in flow obstruction and mass caused by the bars, i.e. they hampered crimpability and flow. Ideally they would remain in order to provide axial stiffness to the stent, but manufacturing limitations prevented the proper mounting of struts. Moreover, the use of Nitinol in prototyping began. An initial prototype was fabricated using 0.019 inch diameter nitinol wire with a new peg board composed of ceramic with $1 / 16$ inch steel 
dowel pins. Ceramic was chosen so that a butane torch could be used to anneal the Nitinol wire on the bench top in order to facilitate rapid prototyping. The results were crude but workable (figure 57). Additionally, a design change in the leaflet attachment was made in order to address leakage flow. A cylindrical cuff of leaflet material was attached to the outside of the proximal stent frame. The outer cuff and inner cuff were sutured together at the based of the PHV using a running loop-stitch. The design was intended to form a seal between the wall of the SA and the stent.

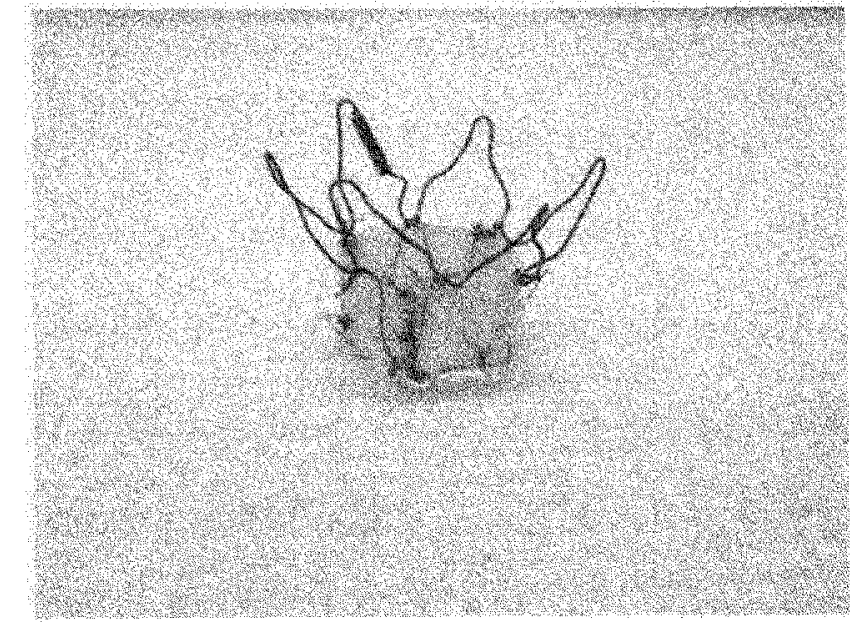

Figure 57: TC PHV 4.0 made with 0.019" OD SEN.

The test results using SA 2.0 showed that the valve was an improvement over TC PHV 2, with an adjusted mean pressure drop of $25 \mathrm{mmHg}$ and a mean regurgitation of $13.5 \%$, which translated to a $19 \%$ decrease in pressure drop and a $36 \%$ decrease in regurgitation compared to TC PHV 2. Crimp testing showed that TC PHV 4 could not fit into the $24 \mathrm{~F}$ slot. 
The favorable results from TC PHV 4 indicated that the PHV design was on track. The next steps were to address pressure drop and crimpability. Concurrently, a thinner walled silicone aorta was designed by adding a modification to SA mold 2.0. The resulting silicone aorta was called SA 2.1 and was used to test TC PHV 4.1 in the LHS. Moreover, the SIBS-Dacron composite leaflet material was used in TC PHV 4.1 instead of the clothing grade polyester fabric. Furthermore, a new peg board was designed for furnace annealing of the nitinol wire. This fabrication method change allowed a standardization of the manufacturing process and produced higher quality parts. TC PHV 4.1 was then constructed using 0.015 inch OD SEN wire (figure 58). Another addition to the manufacturing process was made with this prototype; three 5/16 inch SS ball bearings and an oven set between $60-70^{\circ} \mathrm{C}$ were used to plastically shape the leaflets. Finally, a loose cuff was added to the outside of the base of the stent to reduce regurgitation and encourage leaflet closure in a manner similar to the sinuses of Valsalva.

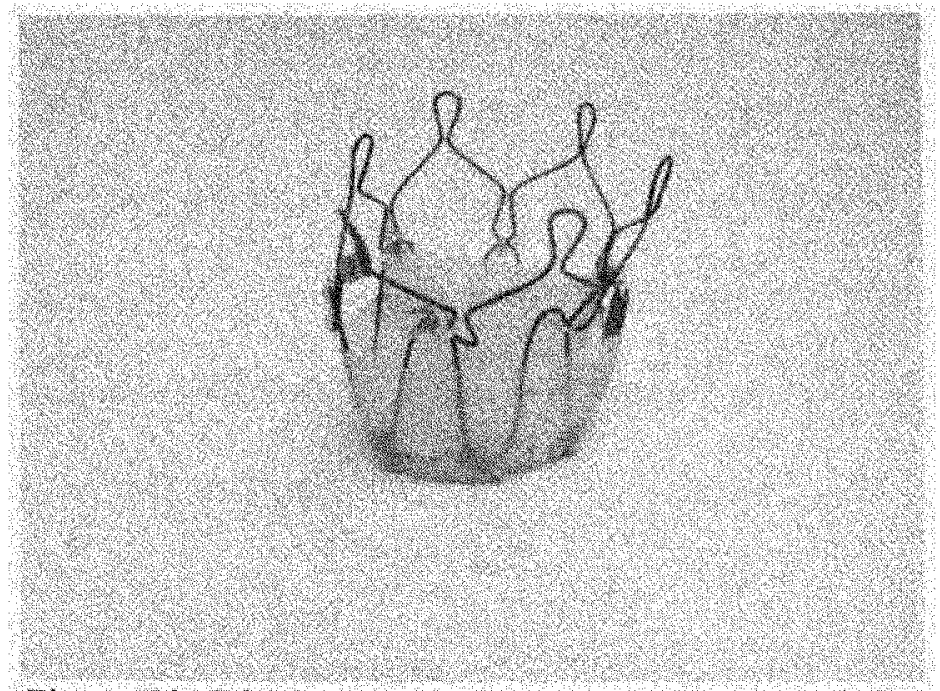

Figure 58: TC PHV 4.1 and leaflet shaping technique. 
The result was an elegant valve that could not hold position during testing. The small diameter wire was too weak to resist the diastolic load and the valve migrated into the ventricle during testing. Therefore, crimpability was moot for this prototype. Only three data sets collected at $45 \mathrm{bpm}$ were recorded before failure (Table 3 ). The adjusted mean pressure drop was $6 \mathrm{mmHg}$ and the mean regurgitation was $5 \%$.

\begin{tabular}{|c|c|c|c|c|c|c|c|c|c|c|c|}
\hline Prototype & HR (bpm) & $\begin{array}{c}\text { Target } \\
\text { co } \\
\text { (Lmin) }\end{array}$ & $\begin{array}{c}\text { Positive } \\
\text { Mean } \Delta P \\
(m m H g)\end{array}$ & $\begin{array}{l}\text { Positive } \\
\text { Max } \\
\text { Pressure } \\
\text { (mmig) }\end{array}$ & $\begin{array}{l}\text { Cardiac } \\
\text { Output } \\
\text { (u/min) }\end{array}$ & $\begin{array}{c}\text { Stroke } \\
\text { Volume } \\
\text { (mL) }\end{array}$ & $\begin{array}{l}\text { Mean } \\
\text { Forward } \\
\text { Flow Rate } \\
\text { (U)wirs) }\end{array}$ & $\begin{array}{l}\text { Leakage } \\
\text { Flow } \\
\text { Volume } \\
\text { (mul) }\end{array}$ & $\begin{array}{c}\text { Mean } \triangle P \\
\text { during } \\
\text { forward } \\
\text { fow } \\
\text { (mmMg) }\end{array}$ & $\begin{array}{c}\text { Regurgitati } \\
\text { on } \%\end{array}$ & $\begin{array}{c}\text { Adju sted Mean } \\
\triangle P \text { during } \\
\text { forward flow } \\
\text { (mmig) }\end{array}$ \\
\hline \multirow[t]{3}{*}{ TC PHV 4.1} & 45 & 23 & 205960 & 385404 & 22720 & 565356 & 86534 & 3.7370 & 135410 & 66100 & 49260 \\
\hline & 45 & 36 & 260588 & 527613 & 35692 & 85.0231 & 115358 & 5.1349 & 200082 & 60334 & 113932 \\
\hline & 45 & 50 & 141175 & 564091 & 50712 & 1159749 & 66809 & 3.3525 & 101960 & 28907 & 15810 \\
\hline mean & 45 & 3.6 & 20.2574 & 49.2369 & 3.6375 & 85.8445 & 8.9567 & 4.0748 & 14.5817 & 5.1800 & 5.9667 \\
\hline
\end{tabular}

Table 3: Data collected for TC PHV 4.1 prior to valve migration into the ventricle.

\subsubsection{TC PHV 4.2}

The lesson learned in TC PHV 4.1 was applied in TC PHV 4.2 by rebuilding the valve with 0.020 inch diameter nitinol wire (figure 59).

TC PHV 4.2 represented a huge leap forward in preventing regurgitation with a mean of $5 \%$, which was a $63 \%$ decrease over TC PHV 4.0 and was directly on target with the design requirements. An observation that affected the next iteration was motion of the base of the valve into the flow stream during systole, i.e. flow was lifting the stent off the wall of the tube with each forward stroke. This may have been the cause of the higher adjusted mean pressure drop of $28 \mathrm{mmHg}$, which was an $11 \%$ increase over TC PHV 4.0. TC PHV 4.2 was crimpable to $24 \mathrm{~F}$. 


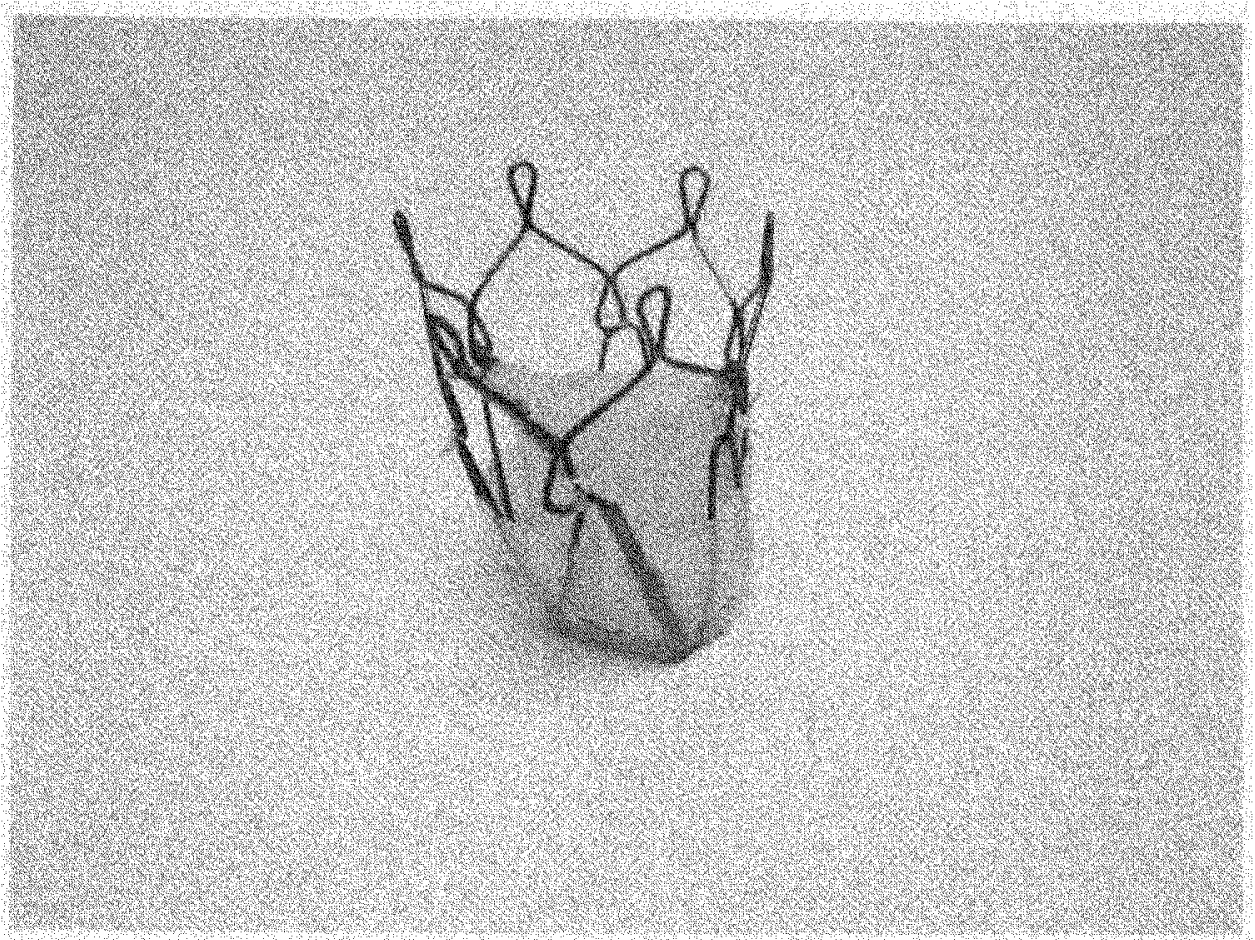

Figure 59: TC PHV 4.2.

\subsubsection{TC PHV 4.3}

Prototype 4.3 was designed to address the phenomenon observed during LHS testing of 4.2. TC PHV 4.2 was observed to have some weakness in the base stent to which the leaflet material was attached; evidenced by a deflection of the stent into the flow stream during systole. Therefore, an additional stent was placed in the base of the PHV (figure 60). The resulting design decreased the adjusted mean pressure drop (14.4 $\mathrm{mmHg}$ ) by $48 \%$ and the mean regurgitation (3.24\%) by $35 \%$ compared to TC PHV 4.2 . These values were directly on target for the design requirements. Additionally, this prototype was crimpable to $24 \mathrm{~F}$ (figure 61 ). 


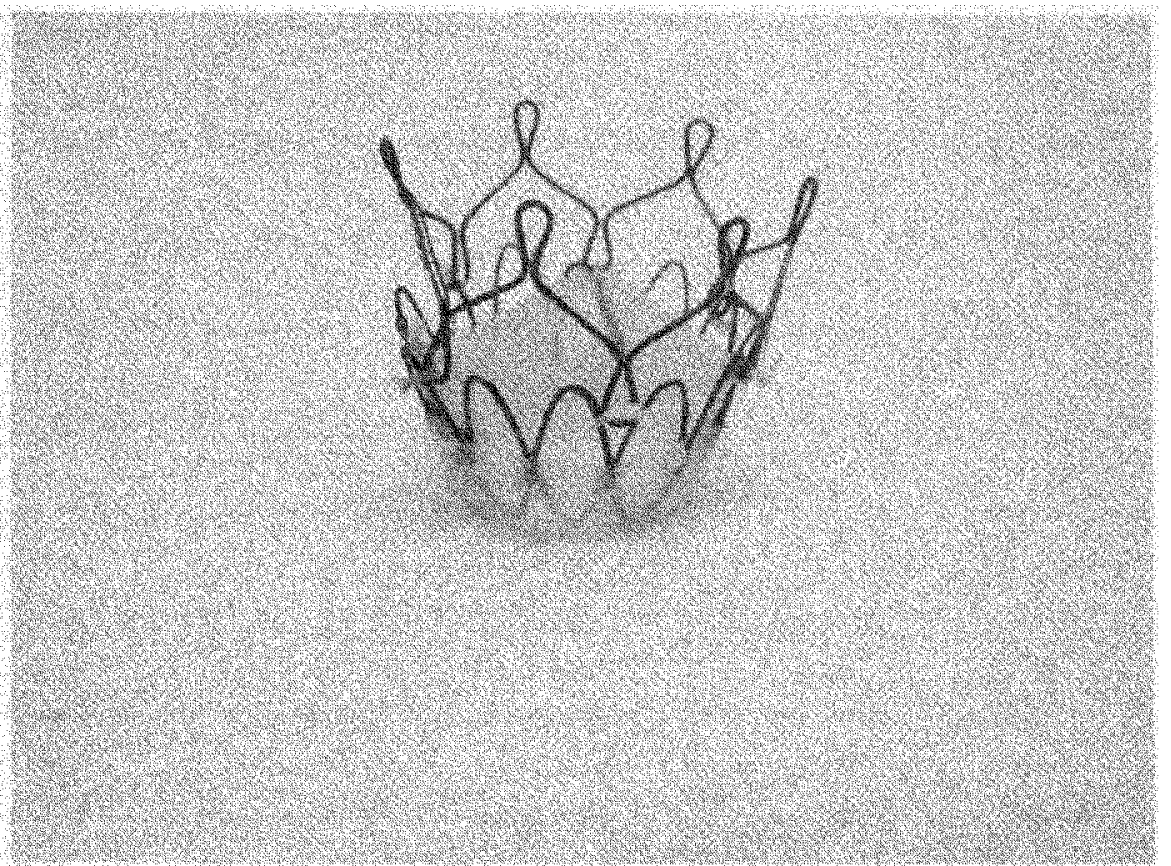

Figure 60: TC PHV 4.3, notice the additional stent in the base of the PHV.

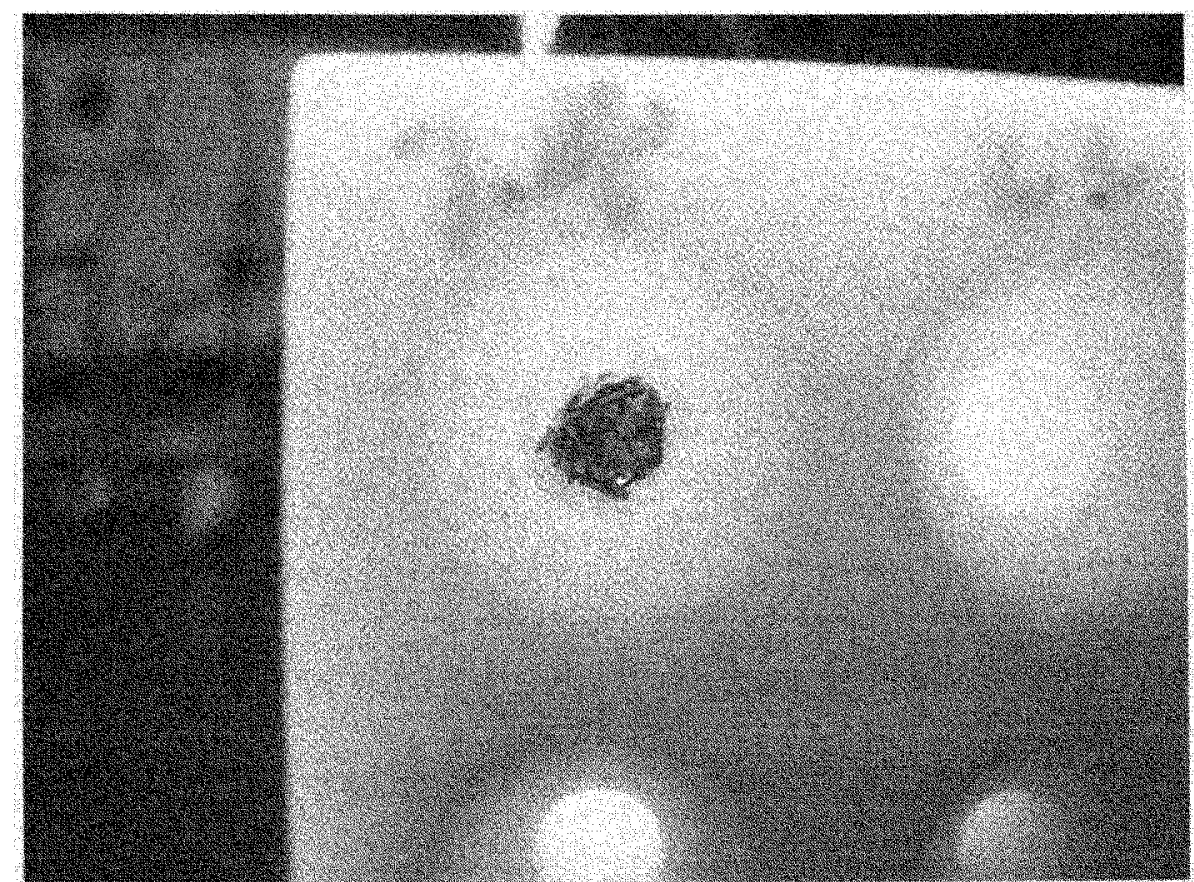

Figure 61: TC PHV 4.3 inside the $24 \mathrm{~F}$ slot of the French Scale block. 
The next prototype was designed to address crimpability. All other design parameters were held constant due to the stellar performance of TC 4.3 in the LHS test. The only design change was a reduction in the leaflet material thickness. Concurrently, the silicone aorta was further modified in order to produce a more compliant arterial model. The wall thickness of the silicone tube was decreased by $50 \%$, then the ratio of elastomer to curing agent was increased, i.e. the curing agent mass was decreased with respect to the mass of the elastomer for each succesive SA $(2.2=10: 1 ; 2.3=12.5: 1$; $2.4=16.67: 1 ; 2.5=25: 1)$. This resulted in a more elastic silicone polymer. By comparison, SA 2.0 was virtually non-compliant and SA 2.1 was about $95 \%$ stiffer than a healthy natural aorta, which exhibits approximately $8-10 \%$ strain with a $40 \mathrm{mmHg}$ pressure change. As a result TC PHV 4.3.1 was tested inside the SA 2.1, 2.2, 2.3, and 2.4. Each SA had increasing values of compliance with SA 2.4 being the most compliant. TC PHV 4.3.1 was successfully crimped down to $21 \mathrm{~F}$.

TC PHV 4.3.1 had a mean adjusted pressure drop of $22 \mathrm{mmHg}$ and a mean regurgitation of $4 \%$ in SA 2.1. In SA 2.2 it had a mean adjusted pressure drop of 24 $\mathrm{mmHg}$ and a mean regurgitation of $9.4 \%$. In SA 2.3 and 2.4 the testing could not be completed due to valve migration and prolapse, but mean pressure drop and regurgitation values of $12 \mathrm{mmHg}$ and $6.3 \%$ and $9.5 \mathrm{mmHg}$ and $13.5 \%$ respectively were recorded prior to failure. It seemed that the more compliant tubing was conducive to valve migration and the many tests endured by TC PHV 4.3.1 illuminated the lack of durability of the design as the leaflet material began to loosen in later tests. Additionally, the more compliant tubing seemed to contribute to an increased volume of leakage flow. 
With TC PHV 4.3.1 being crimpable to $21 \mathrm{~F}$, the next iteration of the prototype was designed to be both crimpable to $21 \mathrm{~F}$ and to have the ability to function in a much more compliant silicone aorta. TC PHV 4.3 .2 (figure 62) was manufactured using the new nitinol wire annealing plates so that the stents would have larger diameters. Greater stent oversizing was to address fixation in an SA with compliance equivalent to a natural healthy aorta. The distal stent was made with 0.020 inch diameter super-elastic nitinol wire, and the proximal two stents were the same type of wire at 0.015 inch diameter. $4-0$ Ethibond Excel suture was used to assemble the PHV. The leaflet material was fabricated using a $3 \times 3$ inch sheet of polyester fabric (Bard) and $8 \mathrm{ml}$ of SIBS-toluene mixture composed of $15 \%$ SIBS by mass. The resulting leaflet material had a thickness of approximately 230 microns. The leaflet material was cut into a $25 \times 65 \mathrm{~mm}$ sheet and made into a cylinder using 4-0 Ethibond Excel suture to join the ends. 304 stainless steel 0.040 inch thin-walled hypodermic tubing and Elmer's Krazy glue were used to join the ends of the wires to make stents. Once the PHV was assembled the leaflets were annealed in a $60^{\circ} \mathrm{C}$ oven for at least two hours using stainless steel ball bearings in order to set a hemispherical shape into the material. TC PHV 4.3.2 was tested in the LHS using SA 2.1, 2.2, 2.3, 2.4. and 2.5. Unfortunately, the valve migrated during testing in each conduit except SA 2.1, the least compliant SA. In SA 2.1 it had an adjusted mean pressure drop during forward flow of $26 \mathrm{mmHg}$ and a mean regurgitant fraction of $11 \%$. Therefore, overall it performed worse than TC PHV 4.3.1. The intended design improvements only served to decrease the performance of the PHV. Moreover, it was not crimpable to $21 \mathrm{~F}$. One more iteration followed in an effort to correct the situation. 
More compliant conduits again seemed to correlate with higher regurgitation and in some cases lower pressure drops.

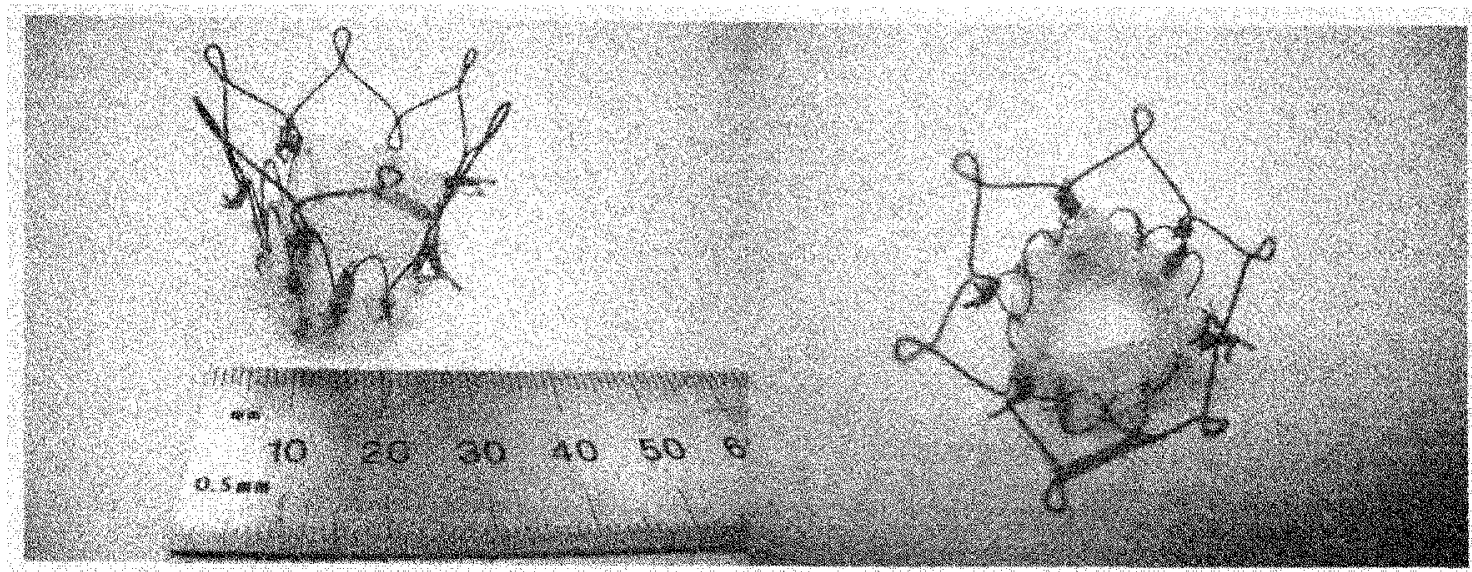

Figure 62: TC PHV 4.3.2

9.3 .11

TC PHV 4.3.3

The last TC PHV prototype fabricated was 4.3.3. TC PHV 4.3.2 was deconstructed and rebuilt as 4.3 .3 (figure 63). Leaflet material was removed to decrease the height of the leaflet material and the amount of overlap of the material on the outer portion of the proximal stents. Additionally, a new suture technique was used to assemble the PHV in an effort to reduce the mass of suture knots around the outer perimeter of the PHV. The valve was tested in SA 2.5 only and it migrated during the first $100 \mathrm{bpm}$ test. Prior to migration an adjusted mean pressure drop of $6.4 \mathrm{mmHg}$ and a mean regurgitant fraction of $21.5 \%$ were recorded. Both TC 4.3 .2 and 4.3 .3 had lower pressure drops and higher regurgitation in the most compliant silicone aorta (2.5). The valve exhibited a relatively large closing volume during testing. 


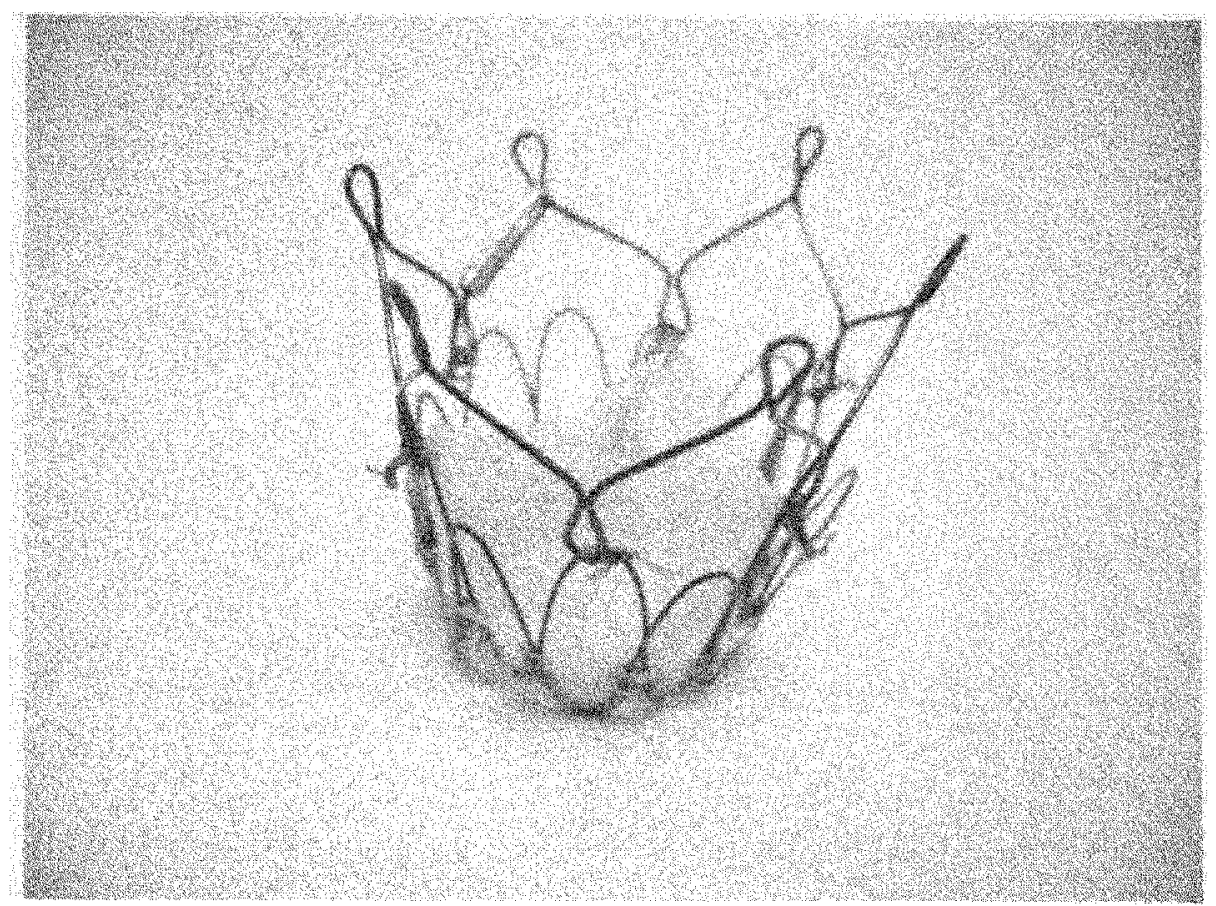

Figure 63: TC PHV 4.3.3.

\subsubsection{EDWARDS LIFE SCIENCES: SAPIEN (CRIBIER)}

In a fortuitous occurrence, an Edwards-Sapien (Cribier) stent was obtained for testing (figure 64). The SIBS-Dacron composite leaflet material was carefully attached to the stent utilizing the predrilled holes in the stent frame. A cuff was made to ensure a tight seal with the tube wall. The result was phenomenal performance. Sapien outperformed all other prototypes and rivaled the performance of the $25 \mathrm{~mm}$ diameter St. Jude bileaflet mechanical valve. The SS ball bearings and $60^{\circ} \mathrm{C}$ oven were again used for leaflet forming. The Sapien set the bar for the final performance of the TC PHV concepts in this proposal, with a mean pressure gradient during forward flow of $15 \mathrm{mmHg}$, and a mean regurgitant fraction of $1.7 \%$. 


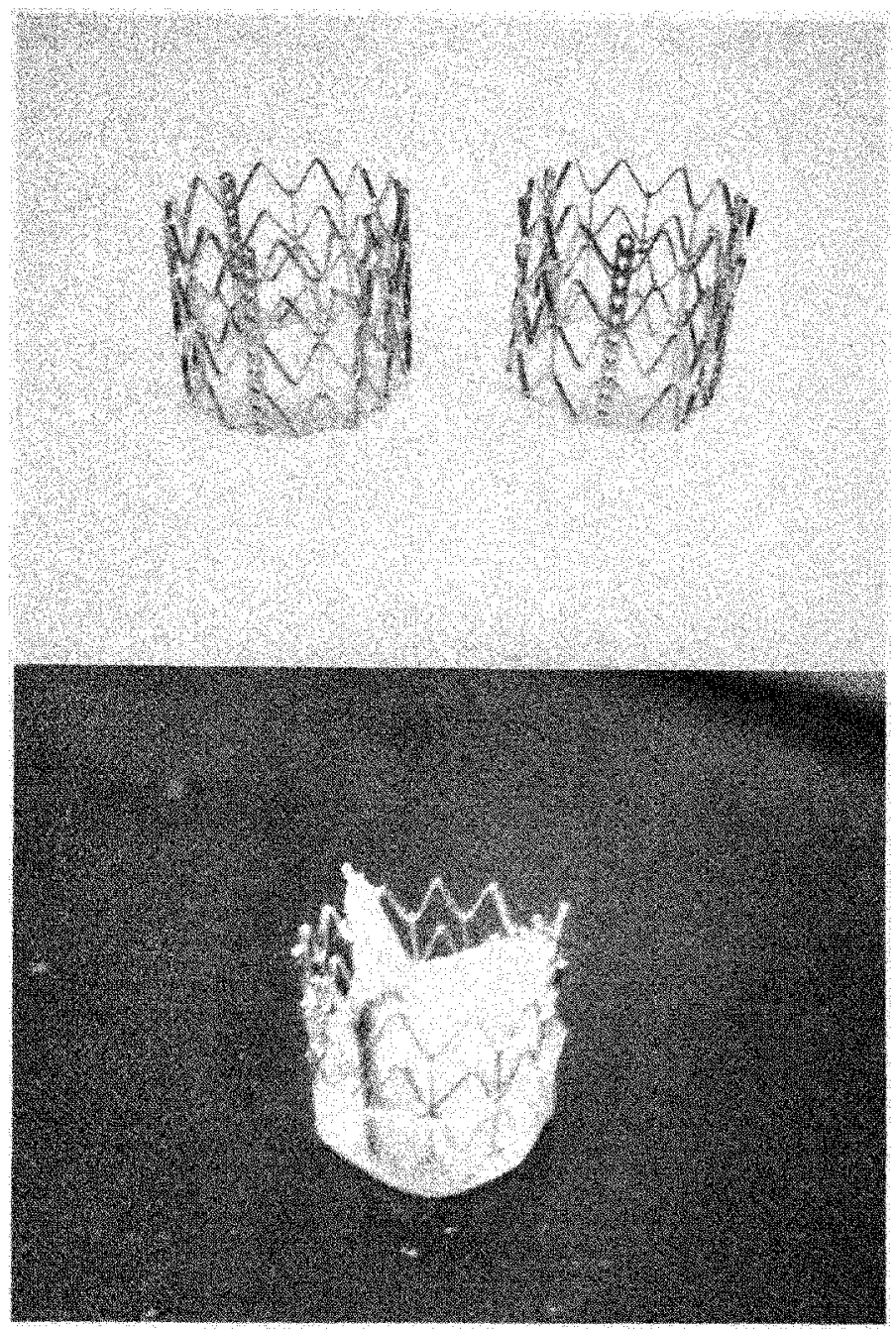

Figure 64: Edwards Life Sciences Sapien valve by Claiborne.

\subsection{HYDRODYNAMIC RESULTS}

Compiled LHS test results are shown plotted in charts 4 and 5. Chart 4 is a plot of cardiac output as function of regurgitation. We expect regurgitation to decrease as cardiac output increases. Data collected for rebuilt FJA-MCB PHV, Sapien, and the Jt. Jude valve were compared to TC PHV 2-4.3. In the cases of TC PHV 4 series, each prototype out performed the rebuilt FJA-MCB PHV. Each successive TC PHV was progressively better than the former as well. TC PHV 4.3 was statistically no different 
than Sapien. Further iteration of the TC PHV 4 series were omitted from these graphs because testing of those valve took place in newer more compliant silicone aortas, which introduced new phenomena that will require further study in the future.

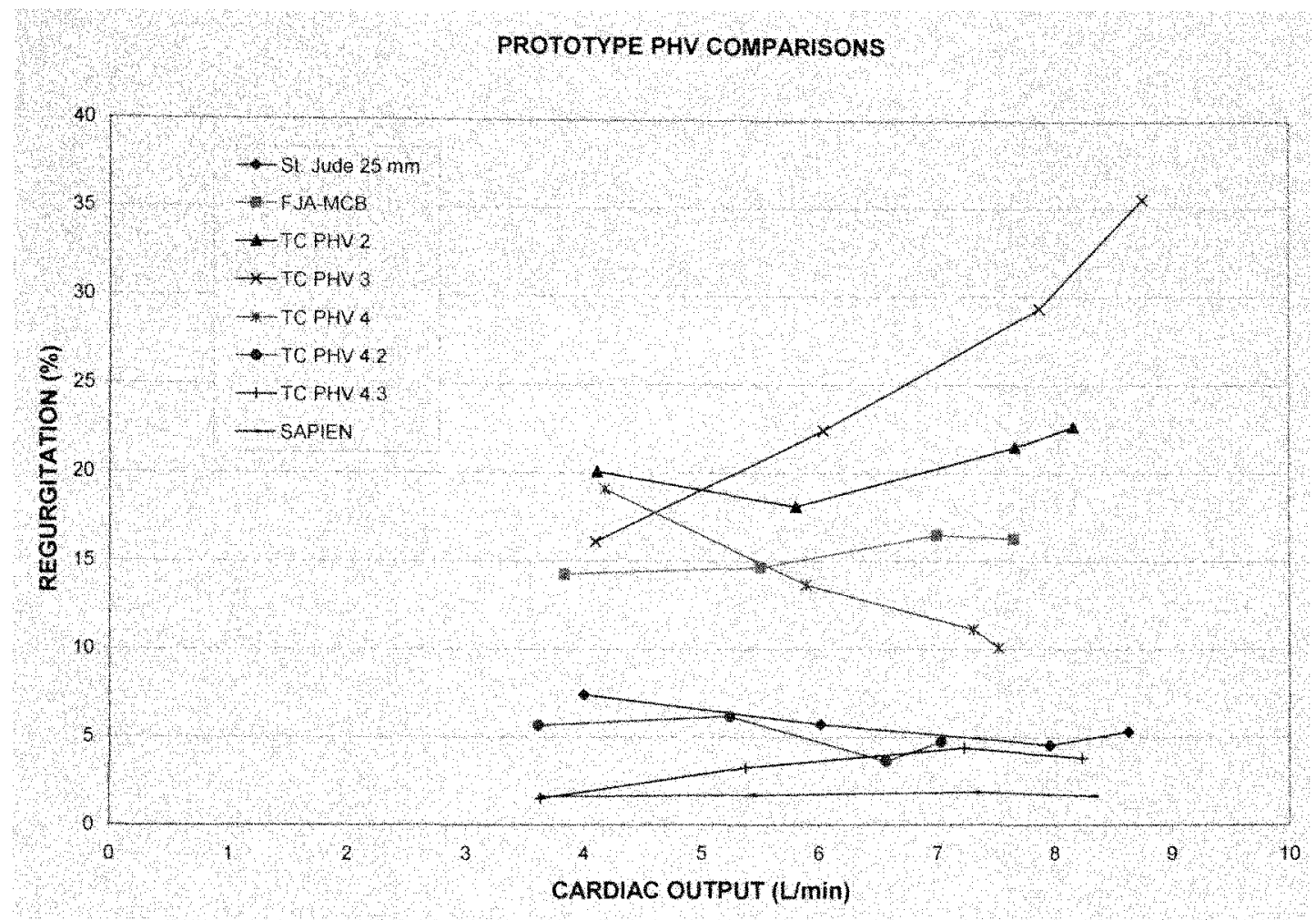

Chart 4: A comparison of valve performance is illustrated with a plot of cardiac output as a function of regurgitation. We expect regurgitation to decrease as cardiac output increases. This chart clearly shows the stepwise improvement in performance with prototype PHV with TC 4.3 having the best recorded performance when compared to Sapien and the St. Jude valve. 


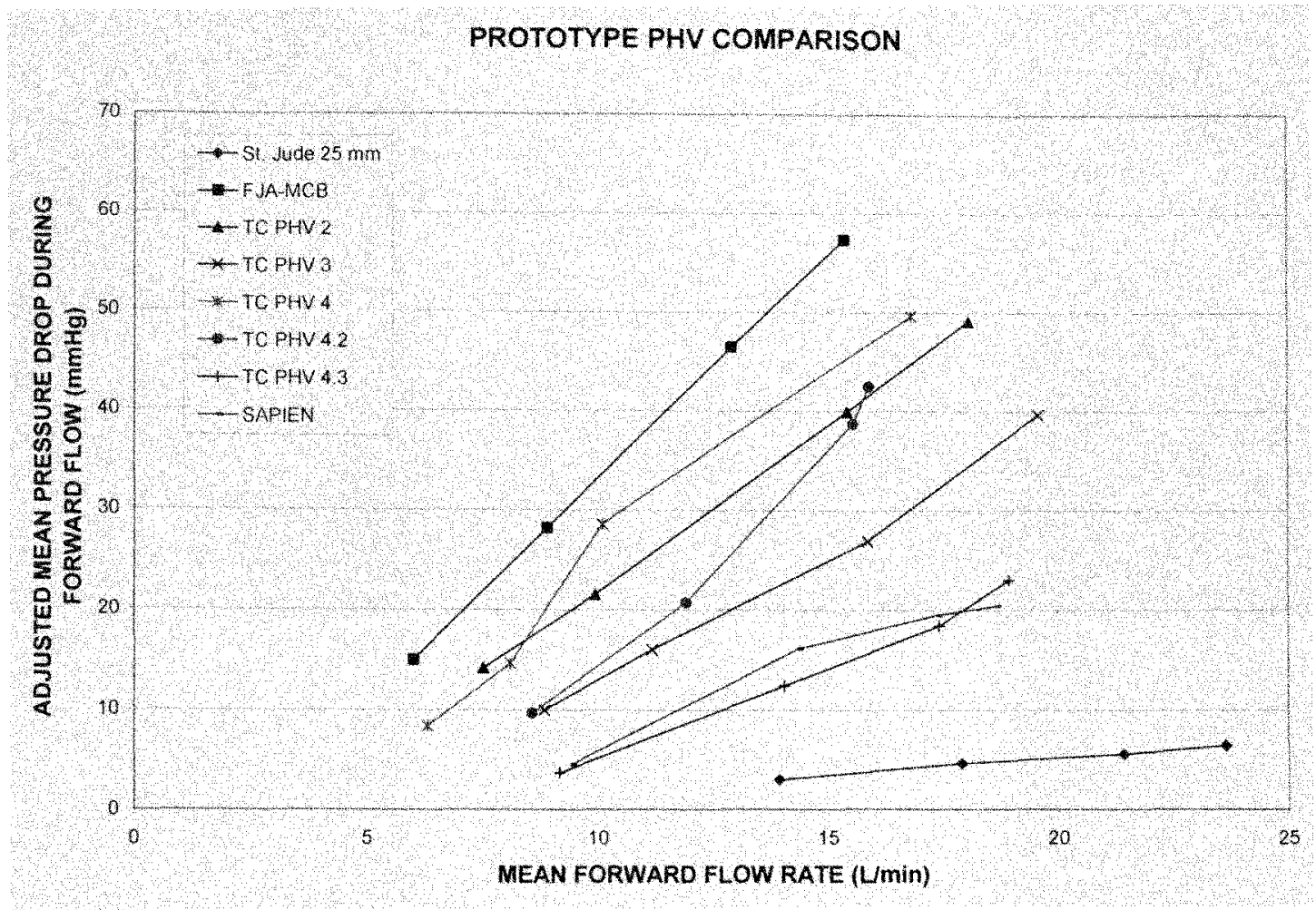

Chart 5: A comparison of prototype performance via a plot of mean forward flow rate as a function of the calculated adjusted mean pressure drop across the valves. As in chart 4 , a clear progression in PHV performance is illustrated as compared to the performance of the Sapien and St. Jude valves. Notably, each tc phv performed better than the rebuilt FJA-MCB valve. We expect an increase in pressure drop as the flow rate increases.

Each silicone aorta utilized for hydrodynamic testing was tested alone in order to measure the intrinsic pressure drop of the conduit. Chart 6 illustrates those results. Oddly, no apparent pattern emerged in the group. Each SA had a different compliance value, but a clear correlation between compliance and the intrinsic pressure drop was not elucidated by the data. 


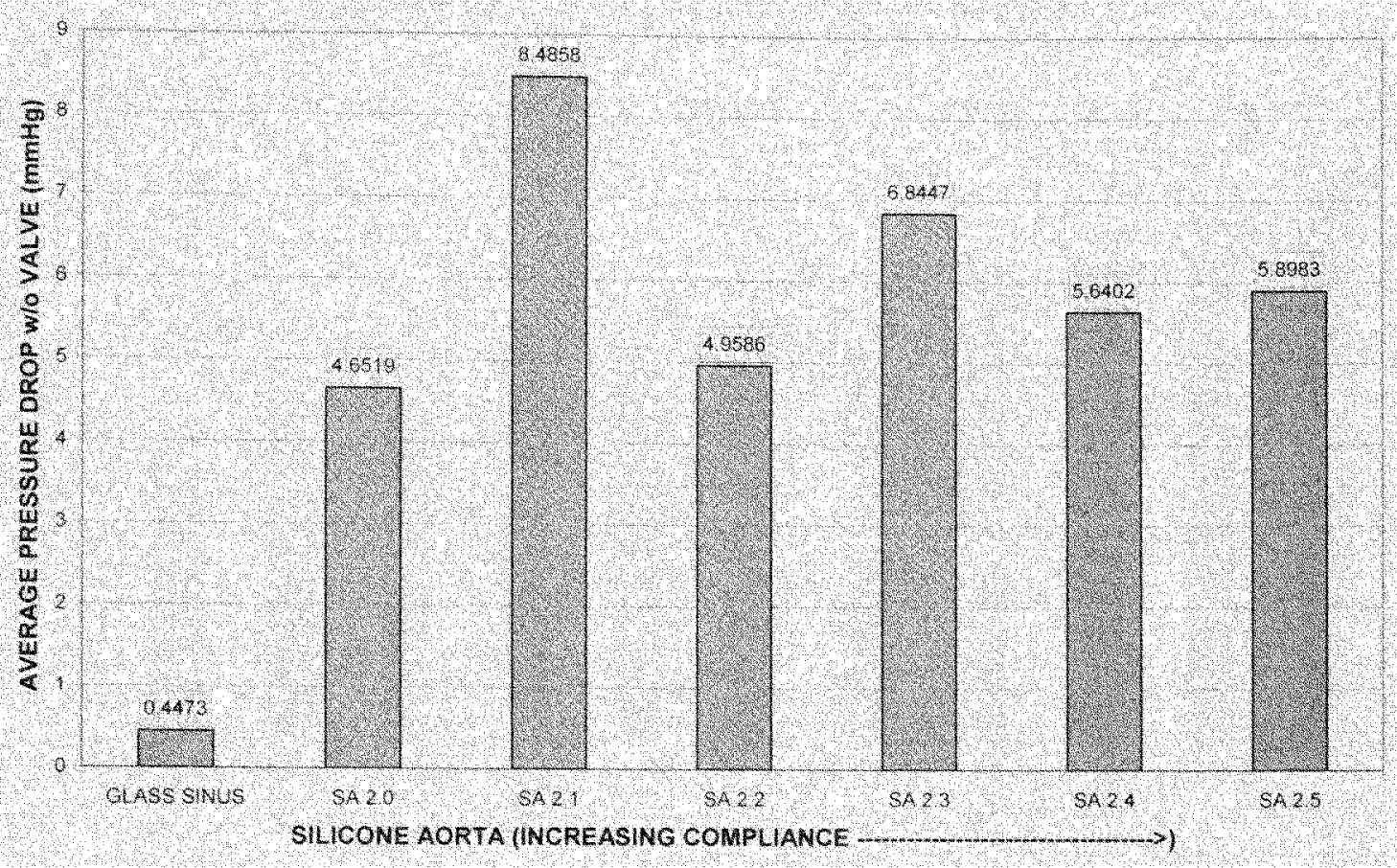

Chart 6: The pressure drop measured across each silicone aorta without a PHV in place. The values shown were used as "tube factors" (TF) to adjust the measured pressure drop across each PHV recorded during each LHS test, i.e. adjusted pressure = raw value - TF. Each successive model was more compliant than the former. The original configuration of the LHS was tested without a valve as well, and the value is noted by the "glass sinus" value $(\mathrm{d}=30 \mathrm{~mm})$. Each $\mathrm{SA}$ had an inner diameter of $19 \mathrm{~mm}$.

The effective orifice area of each prototype was calculated using equation 20 in accordance with ISO 5840:2005. Matlab was used for the calculations and the program can be found in the appendix. The minimum EOA required by the ISO standard for a 19 $\mathrm{mm}$ tissue annulus diameter (TAD) was $0.70 \mathrm{~cm}^{2}$. Each TC PHV surpassed that requirement as shown in chart 7 . TC PHV 4.3 .3 produced the largest EOA when tested in the most compliant SA (2.5). The effect of altering the compliance is illustrated in chart 8 . 
(ISO $5840.200519 \mathrm{~mm}$ TAD requires EOA $>=0.70 \mathrm{~cm}^{\wedge} 2$ )

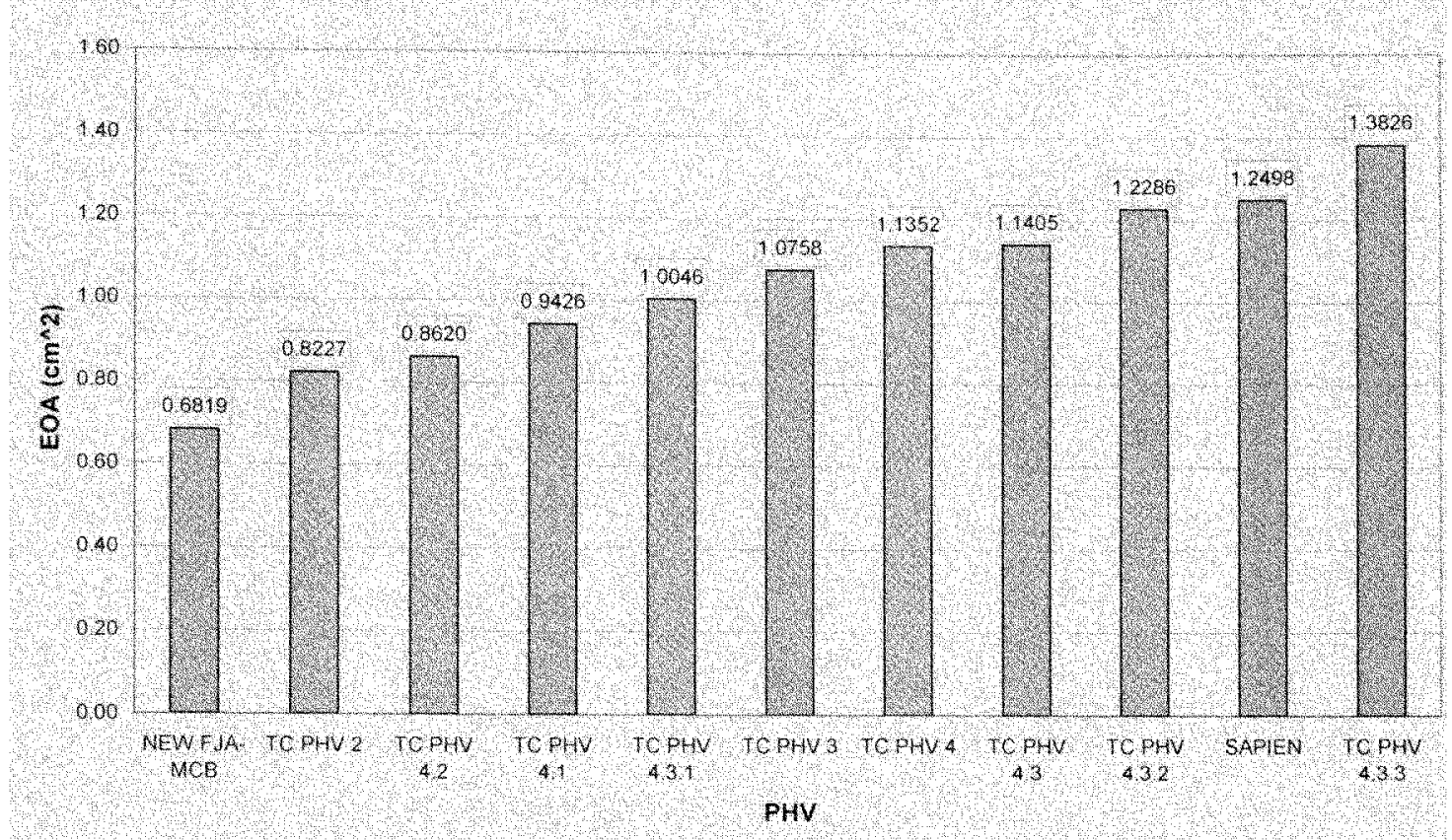

Chart 7: ISO 5840:2005 effective orifice area for each prototype PHV. The EOA minimum requirement for a $19 \mathrm{~mm}$ tissue annulus diameter is $0.70 \mathrm{~cm}^{2}$. Each TC PHV prototype surpassed that requirement. The compliance of the SA had a significant effect on the EOA. The less compliant the SA; the lower the EOA. 


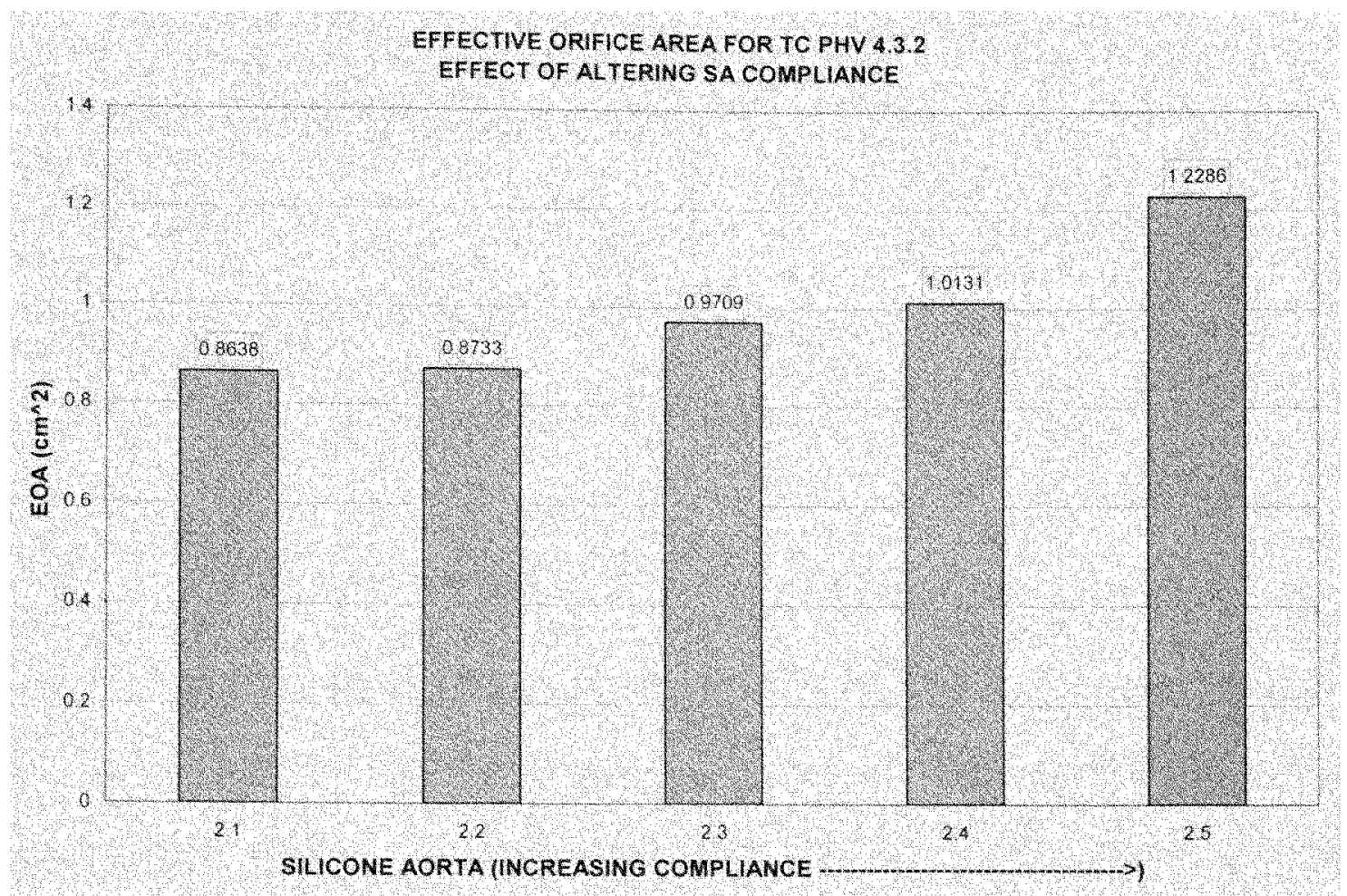

Chart 8: 'The effect of increasing the SA compliance is illustrated by showing that one PHV can have significantly different EOA values as the test conduit compliance changes. This shows the ability of the Nitinol stent to conform to changes in the conduit structure.

Statistical analysis of the hydrodynamic data using one-way ANOVA of means via SPSS 13.0 showed that each PHV prototype was significantly different in terms of adjusted mean pressure drop across the PHV and mean regurgitation fraction ( $p<0.001)$. Additionally, each model of silicone aorta was significantly different in terms of the mean pressure drop across the conduit without a valve in situ $(\mathrm{p}=0.001)$. 


\begin{tabular}{|ll|r|r|r|r|r|}
\hline & & \multicolumn{1}{|c|}{$\begin{array}{c}\text { Sum of } \\
\text { Squares }\end{array}$} & df & Mean Square & F & Sig. \\
\hline Adjusted Mean Fressure & Between Groups & 8869.721 & 9 & 985.525 & 6.302 & .000 \\
Drop During Forward & Within Groups & 17046.108 & 109 & 156.386 & & \\
Flow (mmig) & Total & 25915.829 & 118 & & & \\
\hline Regurgitation (\%) & Between Groups & 6559.560 & 9 & 728.840 & 26.701 & .000 \\
& Within Groups & 2975.294 & 109 & 27.296 & & \\
& Total & 9534.854 & 118 & & & \\
\hline
\end{tabular}

Table 4: SPSS 13.0 one-way ANOVA shown. The means of each parameter of each valve were significantly different $(p<0.001)$.

\subsection{FORCE TESTING RESULTS}

During compression testing a diameter of $19 \mathrm{~mm}$ was reached when the linear displacement was $0.28 \mathrm{~mm}$ in a range of -6 to $+6 \mathrm{~mm}$. The measured force value recorded at that instant was used in the radial force and stiffness calculations because the PHV were designed to reside inside $19 \mathrm{~mm}$ diameter conduits, so the force applied to the conduit wall by the PHV at that diameter was the parameter of interest. Overall, chart 9 showed a positive linear trend in the applied force as the displacement increased. The Sapien compression test was ended prior to the $12 \mathrm{~mm}$ displacement being reached because it had reached a load of $12.5 \mathrm{~N}$ and the Teflon material began to stretch around the Sapien valve rather than crushing the valve. In the case of Sapien alone, a less elastic material would have been needed to perform the test to $12 \mathrm{~mm}$ of displacement. However, this was not pursued because Sapien displayed a radial force at $-3 \mathrm{~mm}$ of displacement 2.62 times greater than TC PHV 2 at $0.28 \mathrm{~mm}$ of displacement. 


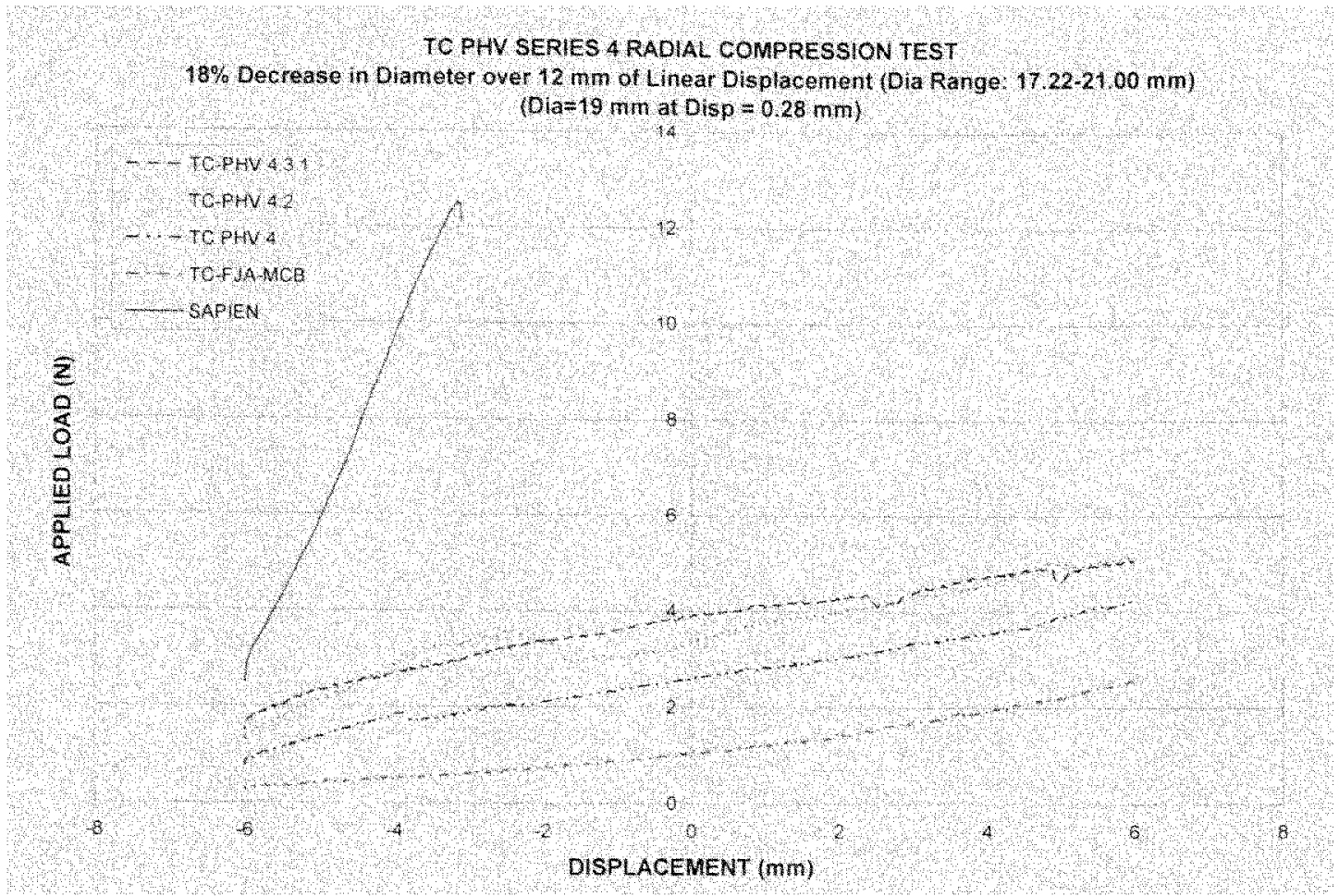

Chart 9: Results from Bose ELF 3200 radial compression tests.

Chart 10 shows the calculated radial force for each PHV prototype tested. By comparison. using equation 28 , an artery with $100 \mathrm{mmHg}$ of internal pressure exerts a radial force of about $0.333 \mathrm{~N} / \mathrm{mm}$. The average length of each PHV prototype was 30 $\mathrm{mm}$, so the radial force applied to the PHV by the artery would be about $10 \mathrm{~N}$. Therefore, the results in chart 13 show that under normal conditions each TC PHV would be able to overcome the crushing force of the artery and dilate its lumen. 


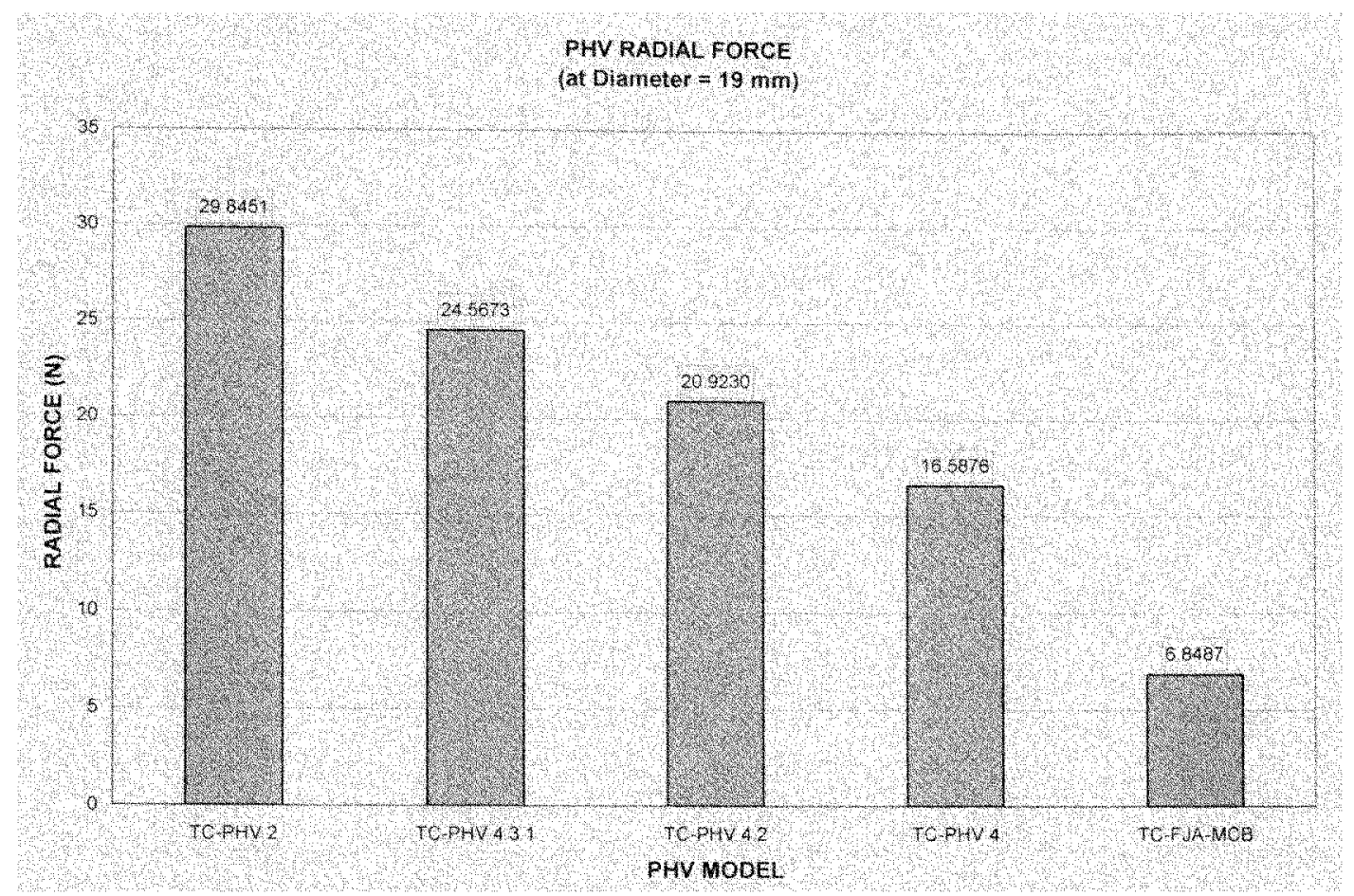

Chart 10: Radial force for each PHV prototype at a diameter of $19 \mathrm{~mm}$, which corresponds to the inner diameter of the silicone aorta used for hydrodynamic testing.

The stiffness of each valve tested was calculated via equation 31 and the results are shown in chart 11. This value was designed to characterize the material properties of the PHV as a whole in terms of its ability to resist crushing for comparison to other types of stents and materials. 


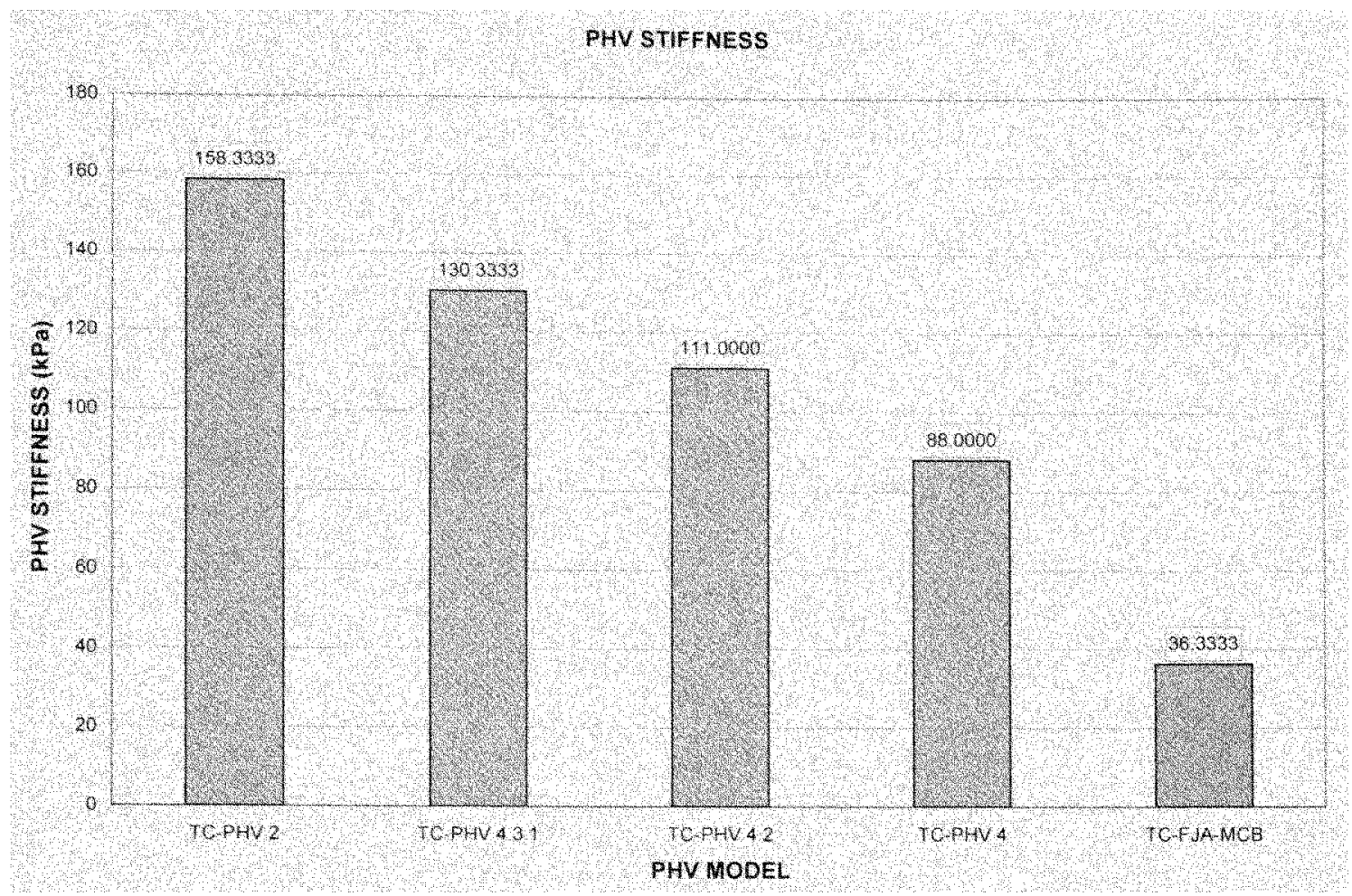

Chart 11: PHV stiffness at a diameter of $19 \mathrm{~mm}$ as defined by Duerig et al. 2000 . Stiffness, $k$, is a material constant that can be universally compared to other stent borne valves.

A stress-strain curve was constructed for the PTFE material used during the compression tests (chart 12). The purpose of during so was to calculate the Young's modulus and to show that the force required to plastically deform the material greater than $6 \mathrm{~N}$, which was not surpassed by any test valve, except Sapien. 


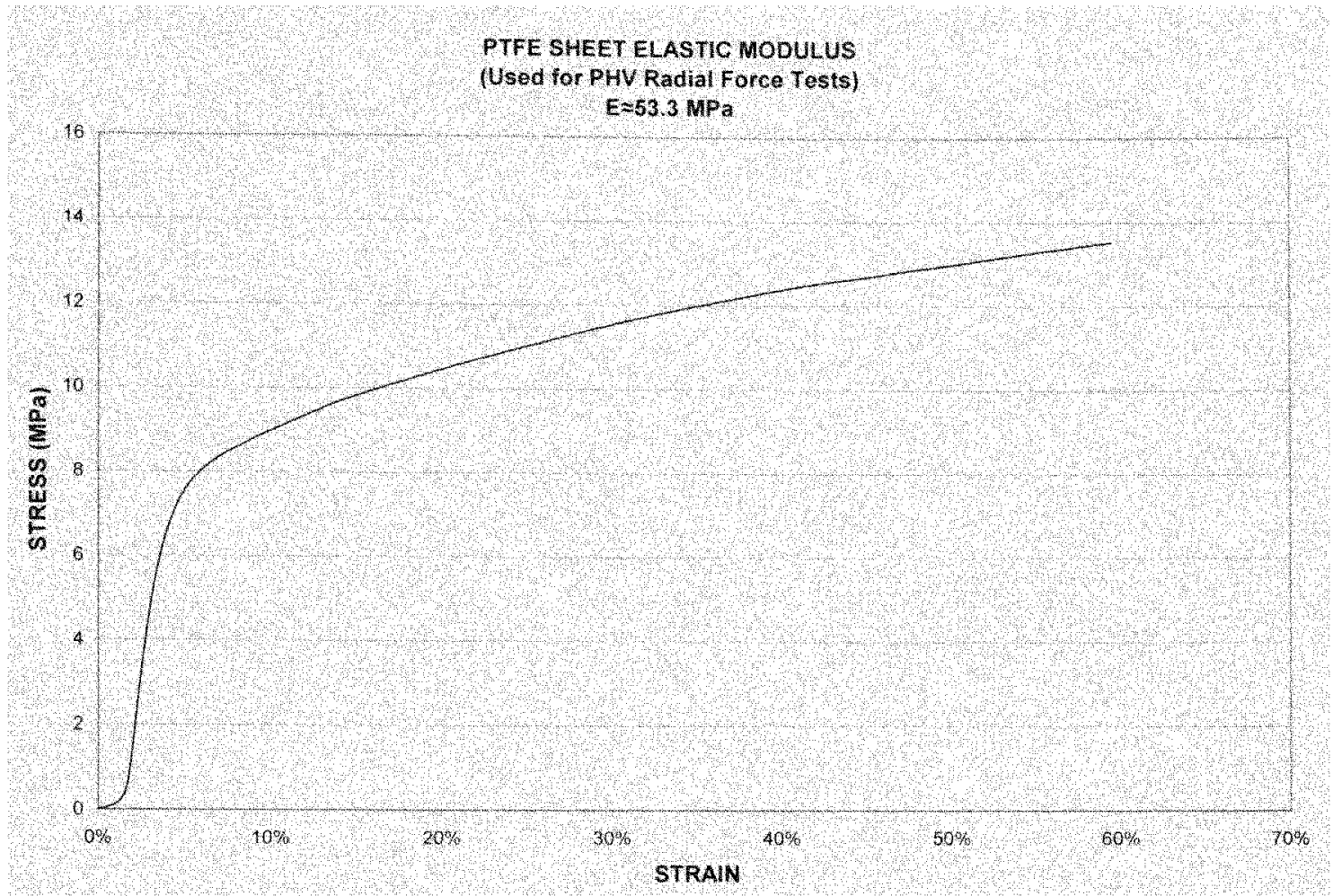

Chart 12: The elastic modulus of the Teflon material used for the PIIV compression tests.

This has been included in order to illustrate that fact that the material could not have exhibited deformation that could have skewed the compression test results. The elastic range of the material is much smaller than Nitinol.

The fixation force test data is plotted in chart 13. Points of initial slippage are marked. Only TC PHV 4 did not exceed the required $3 \mathrm{~N}$ limit, which is equivalent to 80 $\mathrm{mmHg}$ of diastolic pressure on the valve in situ. 


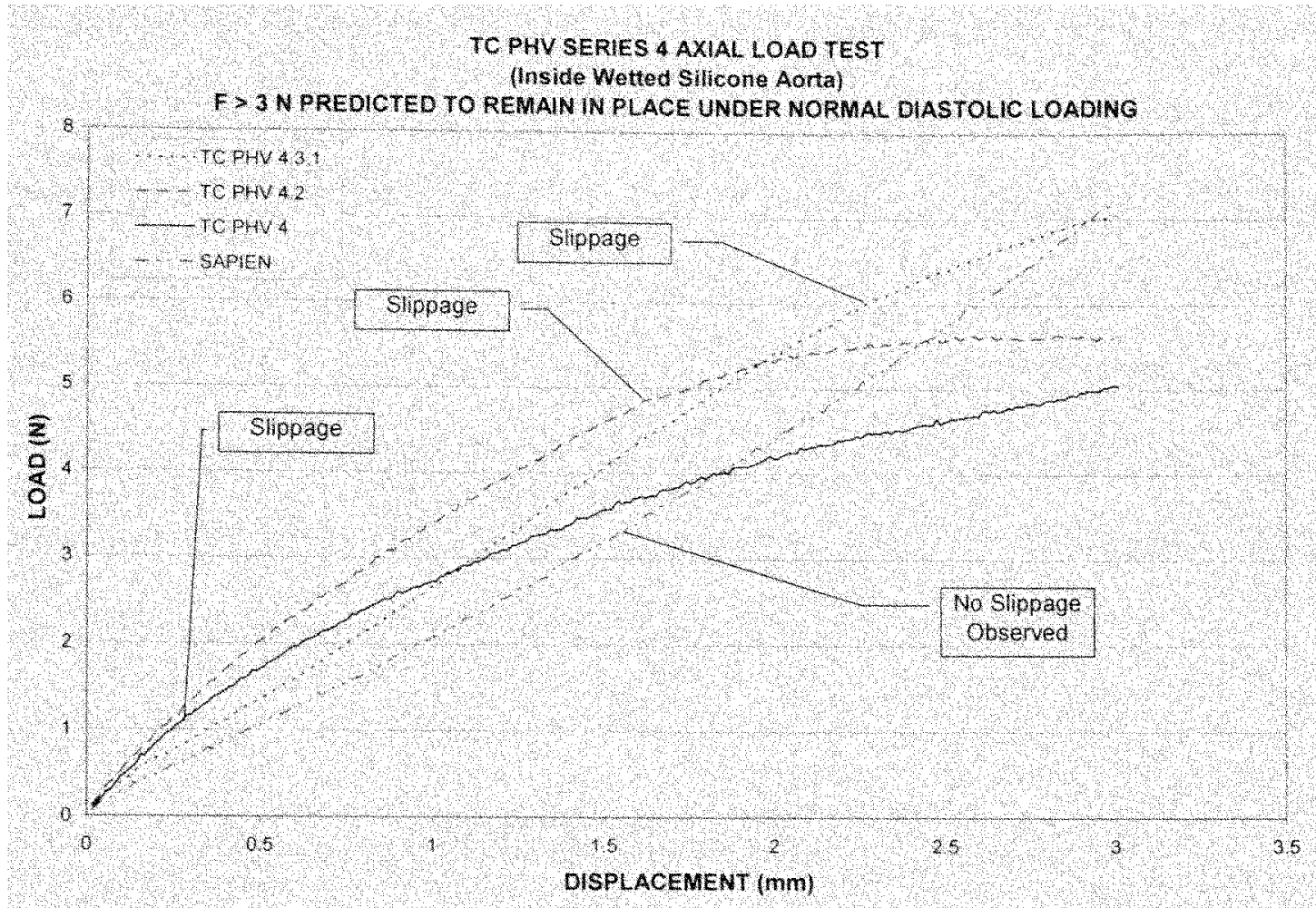

Chart 13: Results from Bose ELF 3200 axial tensile tests. A fixation force greater than 3 $\mathrm{N}$ was considered success. (Diastolic pressure, $80 \mathrm{mmHg}$, exerts approximately $3 \mathrm{~N}$ of force on the PHV. Fluid shear stress was not considered.)

\subsection{COMPLIANCE RESULTS}

The compliance of hydrodynamic test chambers for prosthetic valves is governed by ISO $5840: 2005$ where high compliance is defined as $0.32 \% / \mathrm{mmHg}$ and low compliance is defined as $0.09 \% / \mathrm{mmHg}$. Therefore, efforts were made to meet or exceed the minimum requirements of the standard. Determining the compliance of the silicone aortas was the first step since they were designed to be analog animal aortas. The results of the compliance tests are shown in chart 14. When the Windkessel model was used to calculate the LHS systemic compliance with each SA in situ no real pattern emerged, but the system did appear to be generally less compliant with the silicone aortas when 
compared to its original configuration (chart 15). Overall, the silicone aortas produced "low" compliance results during hydrodynamic testing. However, systemic calculated compliance may have been "low", but the actual change in diameter of each SA during the cardiac cycle of the LHS could be visualized. Interestingly, the bench-top compliance of SA 2.5 was calculated to be $0.208 \% / \mathrm{mmHg}$, but photographic analysis of SA 2.5 in action inside the LHS showed about a $7.4 \%$ change in diameter with a pulse pressure of $160 \mathrm{mmHg}$, which turned out to be a compliance of $0.05 \% / \mathrm{mmHg}$. So the LHS system somehow damped the intrinsic compliance of the SA by $76 \%$.

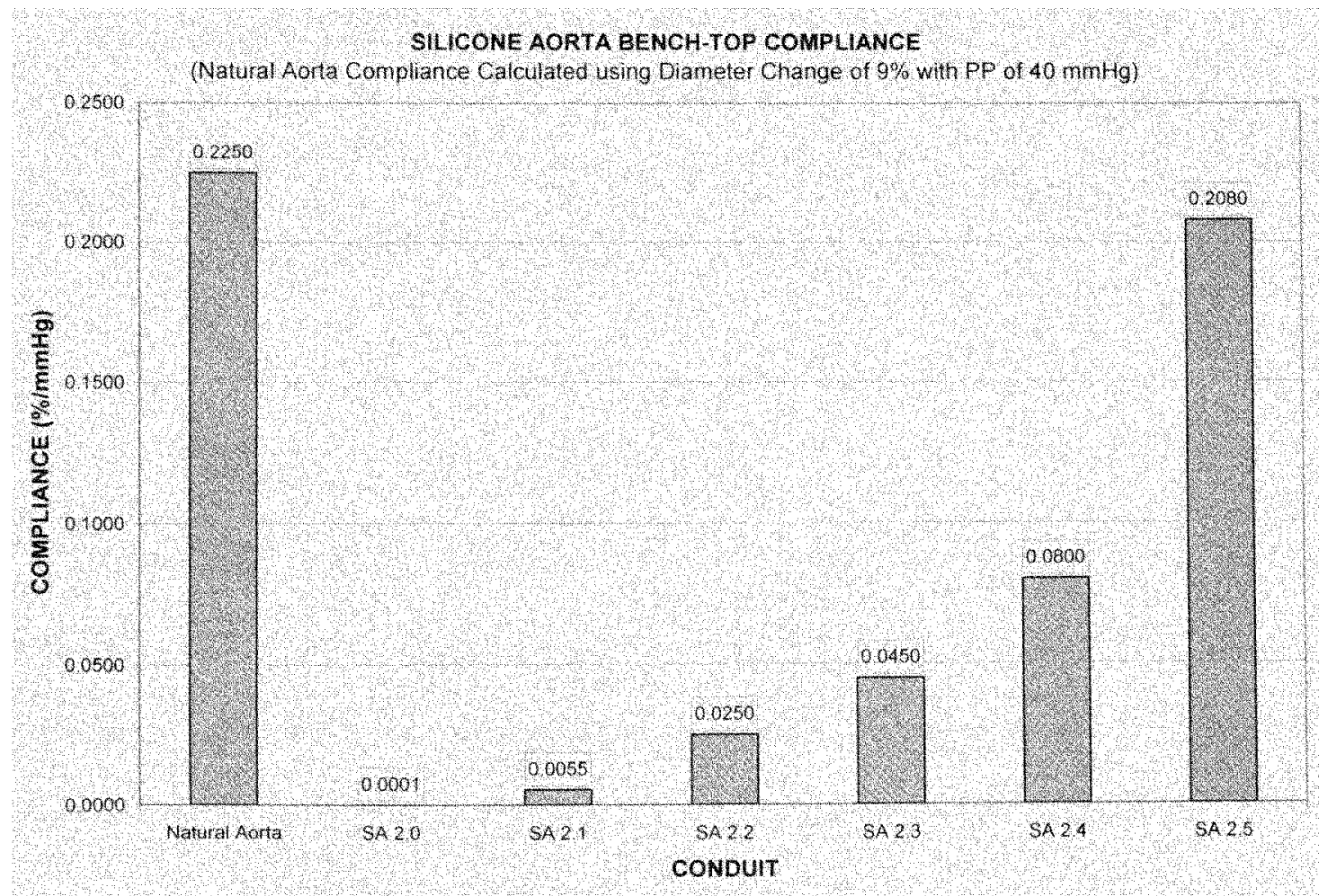

Chart 14: Compliance values measured and calculated on the bench top. The natural aorta value was calculated by assuming a $9 \%$ diameter change with a pulse pressure of 40 mmHg. According to ISO 5840:2005, C=0.09 is a "low" compliance value, whereas $\mathrm{C}=0.32$ is a "high" compliance value. 


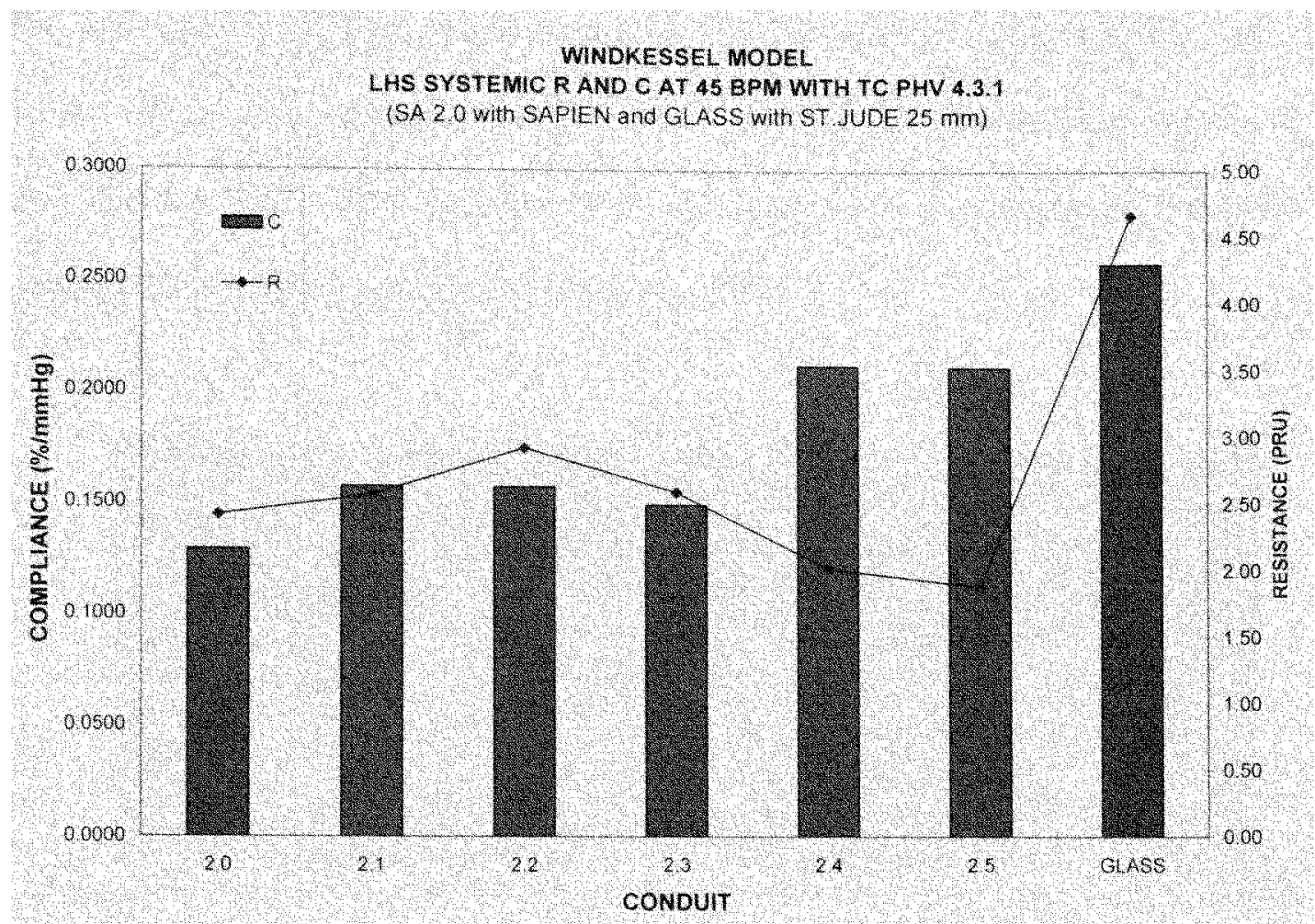

Chart 15: Windkessel model results for LHS systemic compliance using each SA and the original configuration using the glass sinus. No real pattern emerges other than the obvious near "natural" compliance of the LHS obtained while employing the manufacturer's specified configuration.

\subsection{DELIVERY SYSTEM RESULTS}

The PHV delivery system functioned as intended overall. The steering mechanism deflected the catheter tip into the center of the test conduit when it was pulled as shown in figure 65 . The TC PHV 4.3 .1 was successfully loaded into the $21 \mathrm{~F}$ lumen catheter using the crimping tool (figure 66). The model aorta was utilized for the test, and successful deployment of the PHV into the silicone aorta was performed (figure 67). Once deployed, the PHV expanded in place as intended. However, loading and deployment of the PHV was performed with difficulty because of the tight fit of the PHV inside the catheter. The catheter itself kinked when it navigated the curve of the tube. 
The glue used to hold the sheath retraction spool in place failed due to the high force needed to retract the sheath. A mock aortic flow loop was designed and attached to the LHS and a dynamic PHV delivery and deployment test was performed. The PHV was successfully deployed into the SA during that test.

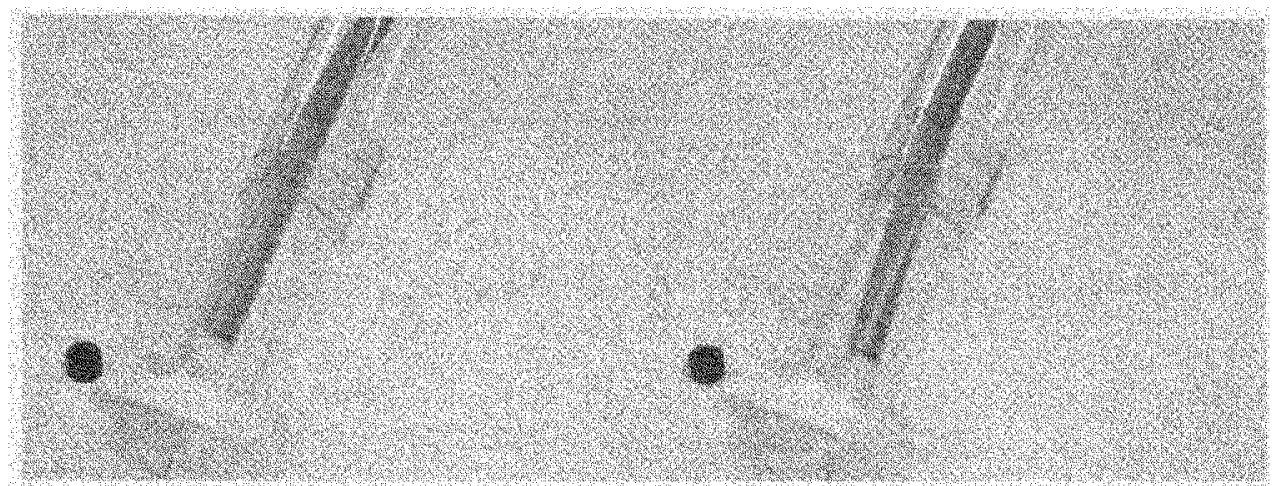

Figure 65: Photos showing the remote deflection of the catheter tip using the catheter hand piece. The tip moved off the tube wall and into the center of the tube using the steering mechanism.

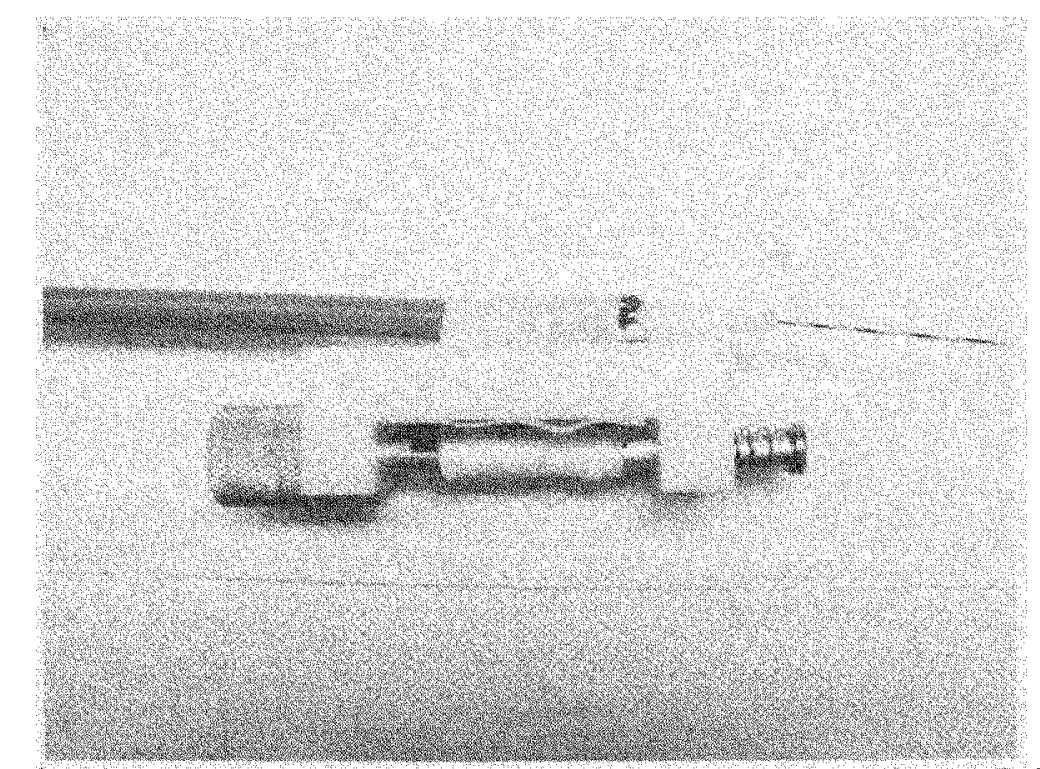

Figure 66: Crimping tool being used to load TC PHV 4.3.1 into the 24 F OD - $21 \mathrm{~F}$ ID catheter tubing prior to the delivery and deployment test. 


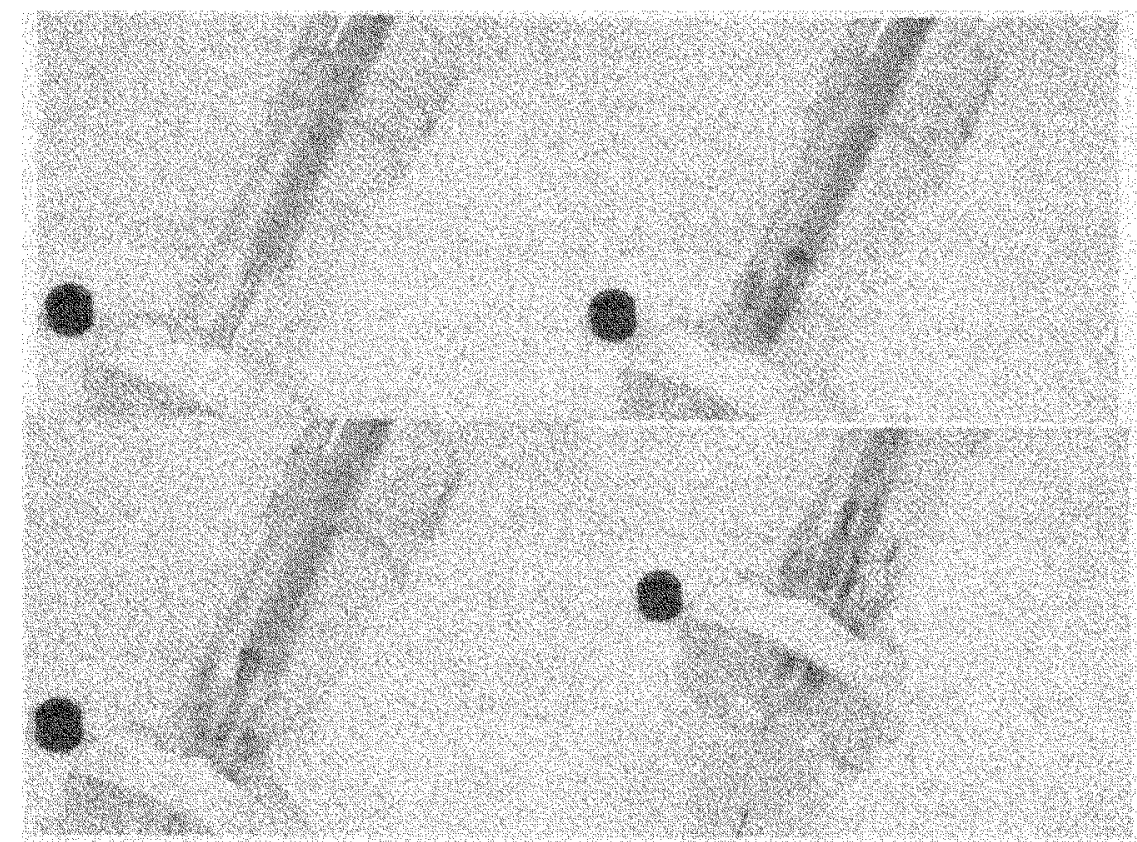

Figure 67: Sequential deployment of the TC PHV 4.3.1 is shown inside the polycarbonate tube and SA 2.4 or the analog aorta as it was.

\section{DISCUSSION}

The current "gold-standard" in heart valve prostheses are mechanical and tissue valves, which are implanted via highly invasive, traumatic, and costly open-heart surgical procedures. Mechanical valves, typified by the St. Jude bileaflet pyrolitic carbon valve, are highly durable but also highly thrombogenic in vivo. Recipients of mechanical valves must endure a lifetime of risky anticoagulant drug therapy. Conversely, tissue valves are less durable and less thrombogenic. As such, tissue valve recipients may require only temporary or mild anticoagulant drug therapy. Therefore, mechanical valves are typically reserved for patients who can tolerate the anticoagulant drugs and who are younger than the age of 65 , and tissue valves are generally reserved for people over the age of 65 or for individuals who would be at greater risk for complications from anticoagulant drugs. Since their invention and commercialization over 30 years ago, 
these two modalities of heart valve disease treatment have seen relatively little change, and neither is ideal. Therefore, there exists opportunity for improvement in the existing technology and creation of new technology to be used as alternative therapy. Catheter deliverable or percutaneous heart valve (PHV) prostheses have been developed recently and promise to be a safe and effective minimally invasive alternative to open-heart valve replacement surgery. There has been great professional interest among interventional cardiologists in PHV technology since the first successful pulmonary PHV human trial by Bonhoeffer et al. in 2000, and the first successful aortic PHV human trial by Cribier et al. in 2002. [20,24] PHV development has rapidly advanced since then primarily in the hands of private industry and physicians. All of the relevant PHVs in development and in human trials are based on tissue valves, which are known to be inferior in terms of durability, and the effects of crimping and deploying tissue PHVs has just begun to be investigated. [33,56] Moreover, the lack of rigorous engineering analysis during development of the current PHVs is becoming evident, e.g. there has been a reported $21 \%$ rate of stent fracture in the Medtronic Melody PHV. [90]

The purpose of this work was to develop and evaluate an artificial aortic PHV with superior characteristics to the current tissue based PHVs, and to develop an accompanying delivery system. It has long been a goal of researchers to develop a prosthetic heart valve which incorporates the positive attributes of both tissue and mechanical valves into one product while eliminating their weaknesses. This concept was extended to PHVs during the course of this work. Recent developments in synthetic heart valve materials have been promising and were a motivating factor. 
The PHV developed during the course of this work utilized a state-of-the-art super-biostable polymer and polyester fabric composite valve material developed at FIU. [58] The stent was manufactured using super-elastic Nitinol wire, and the PHV was assembled using braided polyester suture and stainless steel hypodermic tubing. The PHV was designed to be self-expanding, to function as a trileaflet valve, and to remain in place via spring force and friction. The TC PHV series 4 , the final chosen design, was based upon an amalgam of current PHV designs and information found in literature. The PHV was no less than $30 \mathrm{~mm}$ in length with a valve height of $15 \mathrm{~mm}$. The proximal portion of the PHV was composed of two sinusoidal stents coupled to the valve composite material, and the distal portion was composed of one $50 \%$ larger diameter wire and stent for fixation. These dimensions were derived from the design constraint of a 19 $\mathrm{mm}$ valve diameter, which corresponded to that of a small adult and the animal model. The valve construction was based upon anatomical studies that showed that the aortic valve functioned like a collapsing tube, and as such the material was formed into a cylinder and tethered at three equidistant points on the proximal stents in order to form a near natural trileaflet valve. [10] The valve material thickness was made as thin as possible (between 200 and 230 microns) in order to facilitate crimping, and because the leaflet material thickness was inversely proportional to its flexibility. Nitinol was chosen as a stent material due to its superior elasticity, its biocompatibility, and its tendency to exert a chronic outward force over its entire working life when oversized with respect to the vessel in which it resides. While stainless steel or other rigid alloy stents may exert a higher initial radial force, a Nitinol stent will tend to slowly reshape the lumen of the vessel in which it resides to a larger diameter due to its chronic outward force. [60] 
Furthermore, a less elastic alloy could be reduced in diameter over time by the cyclic loading applied by the muscular arterial wall and the dynamic aortic annulus. The diameter of the Nitinol wire used in the final prototype was chosen based upon experimental results. A larger diameter wire had a higher stiffness, and the tear-drop shaped bends formed in the distal stent increased the overall stiffness. The crimped size of the stent was directly related to the wire diameter and the number of bends in the wire, therefore those parameters were minimized in the design. [59] As a result each TC PHV 4 series was crimpable to $24 \mathrm{~F}$ or less, and the distal stent had $50 \%$ greater wire and stent diameter than the proximal stents. Additionally, the Contraction Limit formula created by FJA predicted a contraction limit of $30 \mathrm{~F}$ for TC PHV 4.3.1, however testing proved that the valve was crimpable to $21 \mathrm{~F}$. [69]

It quickly became apparent upon replicating prior hydrodynamic tests, which employed porcine aortic segments, that a new model and method of testing PHVs in the lab would need to be created. [69] The goal was to increase in vitro test quality control and to eliminate the variability, costs, hazards, and labor akin to in vitro animal blood vessel models. The initial concept was to simply replace the porcine aorta with a transparent silicone tube of equivalent size while maintaining the use of the fixture developed by FJA. However, this still allowed some variability via the fit of the FJA fixture to a silicone tube and the marriage of the two inside the LHS. Moreover, the initial silicone tube mold was less than ideal. In light of those limitations inspiration came in the form of a single solid molded silicone tube that could fit, with optimal precision, inside the LHS without modification of the LHS itself, while eliminating the extra components required by the FJA apparatus. The initial SA (2.0) was designed to fit 
and function inside the LHS without regard to compliance. Once proof of concept had been established, the compliance of the SA was manipulated. This was accomplished via changing the wall thickness and altering the mixture ratio of silicone elastomer to curing agent. The silicone elastomer tubes proved to be adequate analogs to porcine aortas, with the advantages of transparency, ease of manufacture, consistent dimensions, and consistent test results. However, they clearly altered the dynamics of the LHS, insofar as compliance and resistance of the system. The LHS was designed to be used for valve testing precisely as constructed by the manufacturer. The first and most important observation was that the SAs had an intrinsic pressure gradient without a valve in situ. This gradient was measured, averaged, then subtracted from the raw pressure gradient data collected during PHV hydrodynamic testing. The subtraction of this "tube factor" allowed comparison of the data to the St. Jude valve and was a more accurate measurement of the pressure gradient caused by the PHV itself. Later hydrodynamic prototype results showed that highly compliant silicone aortas introduced new flow and pressure phenomena such as an increased closing volume, decreased transvalvular pressure gradient, increased stroke volume and cardiac output, and increased effective orifice area. During hydrodynamic testing using the most compliant SA (2.5) an $8 \%$ diameter change was observed with a pulse pressure of $140 \mathrm{mmHg}$. This was less than the bench top value measured. Therefore, the LHS enclosure must have reduced the SA's compliance in some manner. Additionally, a reduction in the fixation abilities of the PHV was observed at high flow rates. Cumulatively, these results made interpretation of chart 15 difficult and called into question the efficacy of the Windkessel Model employed for modeling the LHS, i.e, the relationship between the compliance of the SA alone and 
the compliance of the LHS as a whole with the SA in situ was not readily apparent. Unfortunately, these observations were made near the end of the project and would need to be addressed in future work. As such, the hydrodynamic test results reported in this work for comparison of PHV performance to the competition omitted the results obtained during testing of PHVs in the highly compliant SAs.

The delivery system was designed to deliver and deploy the PHV retrogradely via peripheral arterial access. The consensus in the medical literature indicated that such an approach was ideal. The catheter tip was designed to be atraumatic to the arterial endothelium and to allow the passage of a guidewire through the lumen of the catheter. The catheter was designed to have an outside diameter of no more than $24 \mathrm{~F}$ and to be stiff enough to push and flexible enough to navigate the tortuous arterial pathway. The inner parts of the catheter were designed to be a framework that would allow remote deflection of the catheter tip for precision positioning and retraction of the sheath for PHV deployment. The hand piece was designed to function as the remote control mechanism for PHV deployment and catheter tip steering. The crimping tool was designed to facilitate crimping and loading of the PHV into the delivery catheter. These design features were recognized as being desirable based upon a review of the literature. Moreover, Edwards Life Sciences had recently developed a steerable PHV catheter for use with its Sapien PHV. [1,34] The prototypes designed and fabricated functioned as intended during bench-top testing and during dynamic delivery and deployment testing performed inside a mock aortic flow loop that was attached to the operating Vivitro Systems, Inc. Left Heart Simulator. However, the FEP tubing used as a catheter was less than ideal in that it kinked when navigating the curvature of the analog aorta. 
Additionally, it took a considerable amount of force (not measured) to retract the catheter sheath due to the tight fit of the PHV inside the $21 \mathrm{~F}$ lumen of the catheter. Unfortunately, there was neither the time nor the budget to optimize the delivery system design. However, for the purposes of this work, the delivery system was a success.

The hydrodynamic test results for each prototype PHV were compared to one another, to a St. Jude $25 \mathrm{~mm}$ diameter bileaflet mechanical valve, and to an EdwardsSapien PHV fabricated with the composite leaflet material. Among the TC PHV prototypes, 4.3 was the best performer. One-way ANOVA showed that the mean performance of each valve was significantly different $(p<0.001)$ when compared as a group. Moreover, Tukey's post hoc test indicated that there was no significant difference between Sapien and TC PHV $4.3(\mathrm{p}=1.000)$, nor between the St. Jude valve and TC PHV $4.3(\mathrm{p}=0.611)$. Additionally, there was no clinical difference between TC PHV 4.3 and the St. Jude valve nor the TC-Sapien PHV. [68] As an aside, the nonnegative slopes of the curves shown in chart 4 could be attributed to the very low resistance of the LHS at high flow rates during those particular tests. In other words, the particular PHV could not achieve high flow rates without there being very low peripheral flow loop resistance. This allowed the valve to open during diastole when the flow was oscillating thereby causing an increase in regurgitation. These statistical results indicated that the TC PHV was at least comparable to competitors.

A review of the literature indicated that a mid-term study of the Sapien PHV showed a mean transvalvular pressure gradient of $10 \mathrm{mmHg}$ with an average valve area of $1.7 \mathrm{~cm}^{2}$, and 0 to +2 regurgitation. [64] TC PHV 4.3 had an adjusted mean pressure drop of $14.4 \mathrm{mmHg}$ with an EOA of $1.14 \mathrm{~cm}^{2}$ and a regurgitation of $3.24 \%$, which is 
essentially 0 on the ACC scale. Both used $24 \mathrm{~F}$ catheters for delivery. When compared to a 30-day trial of the CoreValve where a mean pressure gradient of about $10 \mathrm{mmHg}$ and regurgitation of 0 to +2 was reported, again TC PHV 4.3 compares favorably. [66] Notably, the delivery catheters used by the CoreValve were 21 and $18 \mathrm{~F}$. However, a limitation to these comparisons was that the TC PHV data was entirely in vitro and the literature data was entirely clinical. To date, there are no known relevant in vitro studies available for comparison.

The fixation and radial forces exerted by the PIV prototypes was tested with a Bose ELF 3200. The fixation test was modeled after a study published by Zhou et al. wherein they deployed an abdominal aortic aneurysm stent graft into a segment of bovine aorta and measured the force required for slippage. [63] In this work one of the silicone aortas was utilized in lieu of a bovine aortic segment and the Bose machine was more sophisticated than the force gauge utilized by Zhou. Additionally, the SA was wetted with the blood analog solution used in hydrodynamic testing of the PHVs. The results showed that the TC series 4 PHVs could resist up to $6 \mathrm{~N}$ of force prior to slipping. This translated into the PHV being able to approximately resist up to $160 \mathrm{mmHg}$ of diastolic pressure, which is well above normal physiological diastolic pressures even for hypertensive individuals. The radial force tests also utilized the Bose ELF 3200 , but the apparatus was different. A piece of 0.015 inch thick PTFE sheet was used to create a loop in which pulling the free ends caused the loop diameter to contract. The starting diameter was set to $21 \mathrm{~mm}$ in each test and the linear travel of the system was set to 12 $\mathrm{mm}$, which translated into an $18 \%$ decrease in the loop diameter during testing. The forces recorded precisely when the loop reached $19 \mathrm{~mm}$ in diameter was used to calculate 
the radial force exerted by each PHV tested. The theoretical radial force calculated for a typical artery of $30 \mathrm{~mm}$ in length was found to be approximately $10 \mathrm{~N}$, and the test results for the TC series 4 PHVs more than doubled that value. [60] Therefore, the PHV was predicted to be able to overcome the crushing force of the aorta and expand to the desired diameter or to the elastic limit of the vessel.

\section{CONCLUSION}

A novel catheter deliverable artificial aortic heart valve prosthesis and delivery system were developed and evaluated. The results indicate that the best performing PHV prototype, TC PHV 4.3, has proven the feasibility of this technology by showing equivalence, and in some cases superiority, to the existing PHV technology currently in clinical trials. Specifically, the in vitro hydrodynamic test results have proven that TC PHV 4.3 was not significantly different $(p>0.05$ ) from the CoreValve, the Sapien PHV (both tissue and polymer composite), nor the $25 \mathrm{~mm}$ St. Jude mechanical valve. Additionally, The delivery system functioned as intended by delivering and deploying TC PHV 4.3.1 on the bench top and inside the functioning Vivitro Systems, Inc. Left Heart Simulator. It had the capability of remote catheter tip deflection and PHV deployment and had a maximum profile of $24 \mathrm{~F}$. The accompanying crimping tool was able to crimp and hold the PHV in a crimped conformation for the facilitation of PHV loading into the delivery catheter. Furthermore, the silicone aortas developed for in vitro hydrodynamic testing proved to be sufficient analogs to animal aortic segments, and achieved the goals of providing quality control, customizable compliance, and eliminating waste, cost, labor and hazards of animal tissue models. The force testing performed on the PHVs indicated 
that the TC series 4 PHVs could resist up to $160 \mathrm{mmHg}$ of diastolic pressure loading and more than twice the estimated $10 \mathrm{~N}$ crushing force of a typical artery.

Future work will include further investigation of the effects of highly compliant silicone aortas on the Vivitro Systems, Inc. Left Heart Simulator and the function of the PHV prototypes and consequent optimization of the in vitro hydrodynamic testing model; durability testing and PHV design optimization; in vitro biocompatibility testing; computer modeling of the PHV; delivery system optimization and quantitative testing; and animal testing. 
1. www.edwards.com

2. Emery, R.W, Mettler. E., Nicoloff, D.M., 1979. A new cardiac prosthesis: St. Jude Medical cardiac valve: in vivo results. Circulation, v. 60, pp. 48-54.

3. Chandran, K.B., Yoganathan, A.P., Rittgers, S.E., 2007. Biofluid Mechanics: The Human Circulation. CRC Press, Boca Raton, FL.

4. Starr, A., Fessler, C.L., Grunkemeier, G., He, G-W., 2002. Heart valve replacement surgery: Past, present and future. Clinical and Experimental Pharmacology and Physiology, v. 29, pp 735-738.

5. Tang, G.H.L., Maganti, M., David, T.E., Feindel, C.M., Scully, H.E., Borger, M.A., 2007. Effect of prior valve type on mortality in reoperative valve surgery. Annals of Thoracic Surgery, v. 83, pp. 938-945.

6. Kulik, A, Bedard, P., Lam, B-K., Rubens, F.D., Hendry, P.J., Masters, R.G., Mesana, T.G., Ruel, M., 2006. Mechanical versus bioprosthetic valve replacement in middle-aged patients. European Journal of Cardio-thoracic Surgery, v. 30, pp. 485-491.

7. Gao, G., Wu, Y., Grunkemeier, G.L., Furnay, A.P., 2004. Durability of pericardial versus porcine aortic valves. Journal of the American College of Cardiology, v. 44 , no. 2 , pp. $384-388$.

8. Rosamond, W., Flegal, K., Friday, G., Furie, K., Go, A., Greenlund, K., Haase, N., Ho, M., Howard, V., Kissela, B., Kittner, S., Lloyd-Jones, D., McDermott, M., Meigs, J., Moy, C., Nichol, G., O'Donnell, C.J., Roger, V., Rumsfeld, J., Sorlie, P., Steinberger, J., Thom, T., Wasserthiel-Smoller, S., Hong, Y., 2007. Heart Disease and Stroke Statistics-2007 Update: A Report from the American Heart Association Statistics Committee and Stroke Statistics Subcommittee. Circulation, v. 115, pp 69-171.

9. "http://www.nlm.nih.gov/medlineplus/ency/article/002954.htm" From: Medline Plus by the US National Library of Medicine and the National Institutes of Health. Article Updated By: J.A. Lee, M.D., Division of Surgery, UCSF, San Francisco, CA. Article Review Provided by VeriMed Healthcare Network. Quality Certified by ADAM, Inc.

10. Thubrikar, Mano, 1990. The Aortic Valve. CRC Press, Inc., Boca Raton, FL.

11. Martini, F.H., 1995. Fundamentals of Anatomy \& Physiology $3^{\text {rd }}$ Edition. Prentice Hall, Upper Saddle River, N.I. 
12. Davies, H., 1965. Catheter mounted valve for temporary relief of aortic insufficiency. The Lancet, v.285, issue 7379, p 250.

13. Moulopoulos, S. D., Anthopoulos, L., Stamatelopoulos, S., Stefadouros, M., 1971. Catheter-mounted aortic valves. Annals Thoracic Surgery, v. 11, no. 5, pp 423-430.

14. Phillips, S.J., Ciborski, M., Freed, P.S., Cascade, P.N., Jaron, D., 1976. A temporary catheter-tip aortic valve: Hemodynamic effects on experimental acute aortic insufficiency. The Annals of Thoracic Surgery, v.21, no.2, pp 134-137.

15. Lababidi, Z., Wu, J-R., Walls, J.T., 1984. Percutaneous balloon aortic valvuloplasty: results in 23 patients. American Journal of Cardiology, v. 53, pp 194-197.

16. Andersen, H. R., Knudsen, L.L., Hasenkam, J.M., 1992. Transluminal implantation of artificial heart valves. Description of a new expandable aortic valve and initial results with implantation by catheter technique in closed chest pigs. European Heart Journal, v. 13, no. 5, pp 704-708.

17. Pavenik, D., Wright, K.C., Wallace, S., 1992. Development and initial experimental evaluation of a prosthetic aortic valve for transcatheter placement. Work in progress. Radiology, v. 183, no. 1, pp 151-154.

18. Pavcnik, D., Kaufman, J., Uchida, B., Correa, L., Hiraki, T., Kyu, S.C., Keller, F.S., Rosch, J., 2004. Second-generation percutaneous bioprosthetic valve: a short term study in sheep. Journal of Vascular Surgery, v. 40, no. 6, pp 1223-1227.

19. Moazami, N., Bessler, M., Argenziano, M., Choudhri, A.F., Cabreeriza, S.E., Allendorf, J.D.F., Rose, E.A., Oz, M.C., 1996. Transluminal aortic valve placement: feasibility study with a newly designed collapsible aortic valve. ASAIO Journal, v. 42, pp M381-M385.

20. Bonhoeffer, P., Boudjemline, Y., Saliba, Z., Merckx, J., Aggoun, Y., Bonnet, D., Acar, P., Le Bidois, J., Sidi, D., Kachaner, J., 2000. Percutaneous replacement of pulmonary valve in a right-ventricle to pulmonary-artery prosthetic conduit with valve dysfunction. Lancet, v. 356, pp 1403-05.

21. Bonhoeffer, P., Boudjemline, Y., Saliba, Z., Hausse, A.O., Aggoun, Y., Bonnet, D., Sidi, D., Kachaner, J., 2000. Transcatheter implantation of a bovine valve in pulmonary position: A lamb study. Circulation, v. 102, pp 813-816.

22. Sochman, J., Peregrin, J. H., Pavcnik, D., Timmermans, H., Rosch, J., 2000. Percutaneous transcatheter aortic disc valve prosthesis implantation: A feasibility study. Cardiovascular and Interventional Radiology v. 23, pp 384-388. 
23. Sochman, J., Peregrin, J.H., Rocek, M., Timmermans, H.A., Pavcnik, D., Rosch, J., 2006. Percutaneous transcatheter one-step mechanical aortic disc valve prosthesis implantation: a preliminary feasibility study in swine. Cardiovascular and Interventional Radiology, v. 29, pp 114-119.

24. Cribier, A., Eltchaninoff, H., Bash, A., Borenstein, N., Tron, C., Bauer, F, Derumeaux, G., Anselme, F., Laborde, F., Leon, M., 2002. Percutaneous transcatheter implantation of an aortic valve prosthesis for calcific aortic stenosis: First human case description. Circulation, v. 106, pp 3006-8.

25. Paniagua, D., Condado, J.A., Besso, J., Velez, M., Burger, B., Bibbo, S., Cedeno, D., Acquatella, H., Mejia, C., Induni, E., Fish, R.D., 2005. First human case of retrograde transcatheter implantation of an aortic valve prosthesis. Texas Heart Institute Journal, v. 32, pp 393-398.

26. Garay, F., Webb, J., Hijazi, Z.M., 2006. Percutaneous replacement of pulmonary valve using the Edwards-Cribier percutaneous heart valve: First report in a human patient. Catheterization and Cardiovascular Interventions, v. 67, pp 659-662.

27. Webb, J.G., Munt, B., Makkar, R.R., Naqvi, T.Z., Dang, N., 2004. Percutaneous stent-mounted valve for treatment of aortic or pulmonary valve disease. Catheterization Cardiovascular Interventions, v. 63, pp 89-93.

28. Ferrari, M., Figulla, H.R., Schlosser, M., Tenner, I., Frerichs, I., Damm, C., Guyenot, V., Werner, G.S., 2004. Transarterial aortic valve replacement with a self expanding stent in pigs. Heart, v. 90, pp 1326-1331.

29. Grube, E., Laborde, J.C., Zickmann, B., Gerckens, U., Felderhoff, T., Sauren, B., Bootsveld, A., Buellesfeld, L., Iversen, S., 2005. First report on human percutaneous transluminal implantation of a self-expanding valve prosthesis for interventional treatment of aortic valve stenosis. Catheterization and Cardiovascular Interventions v. 66, pp 465-469.

30. Lutter, G., Kuklinkski, D., Berg, G., von Samson, P., Martin, J., Handke, M., Uhrmeister, P., Beyersdorf, F., 2002. Percutaneous aortic valve replacement: An experimental study. I. Studies on implantation. Journal of Thoracic and Cardiovascular Surgery, v. 123, pp 768-776.

31. Lutter, G., Ardehali, R., Cremer, J., Bonhoeffer, P., 2004. Percutaneous Valve Replacement: Current State and Future Prospects. The Annals of Thoracic Surgery, v. 78 , no. 6 , pp 2199-2206.

32. Attman, T., Jahnke, T., Quaden, R., Boening, A., Muller-Hulsbeck, S., Cremer, J., Lutter, G., 2005. Advances in experimental percutaneous pulmonary valve replacement. Annals of Thoracic Surgery, v. 80, pp 969-975. 
33. Attmann, T., Quaden, R., Freistedt, A., Konig, C., Cremer, J., Lutter, G., 2007. Percutaneous heart valve replacement: histology and calcification characteristics of biological valved stents in juvenile sheep. Cardiovascular Pathology, v.16, pp $165-170$.

34. Attmann, T., Lutter, G., Quaden, R., Jahnke, T., Rumberg, K, Cremer, J, MullerHulsbeck, S., 2006. Percutaneous valve replacement: Significance of different delivery systems in vitro and in vivo. Cardiovascular Interventional Radiology, v. 29 , no. 3, pp 406-412.

35. Attmann, T., Quaden, R., Jahnke, T., Muller-Hulsbeck, S., Boening, A., Cremer, J., Lutter, G., 2006. Percutaneous pulmonary valve replacement: 3-month evaluation of self-expanding valved stents. The Annals of Thoracic Surgery, v. 82 , no. 2 , pp 708-713.

36. Attmann, T., Steinseifer, U., Cremer, J., Lutter, G., 2006. (Images in cardiothoracic surgery) Percutaneous valve replacement: a novel low-profile polyurethane valved stent. European Journal of Cardio-Thoracic Surgery, v. 30, p 379.

37. Quaden, R., Attmann, T., Boening, A., Cremer, J., Lutter, G., 2005. Percutaneous aortic valve replacement: Resection before implantation. European Journal of Cardio-Thoracic Surgery, v. 27, pp 836-840.

38. Quaden, R., Klaws, G-R., Theisen-Kunde, D., Lutter, G., 2007. (Images in cardiothoracic surgery) Percutaneous aortic valve replacement: first endovascular resection of human aortic valves in situ. European Journal of Cardio-Thoracic Surgery, v. 31, p 305.

39. Quaden, R., Klaws, G-R., Theisen-Kunde, D., Lutter, G., 2007. Images in cardiothoracic surgery. Percutaneous aortic valve replacement: first endovascular resection of human aortic valve in situ. European Journal of Cardio-Thoracic Surgery, v. 31, p 305

40. Scheiber, C., Horer, J., Vogt, M., Fratz, S., Kunze, M., Galm, C., Eicken, A., Lange, R., 2007. A new treatment option for pulmonary valvular insufficiency: First experiences with implantation of a self-expanding stented valve without use of cardiopulmonary bypass. European Journal of Cardio-Thoracic Surgery, v. 31, pp 26-30.

41. Leon, M.B., Kodali S., Williams, M., Oz, M., Smith, C., Stewart, A., Schwartz, A., Collins, M., Moses, J.W., 2006. Transcatheter aortic valve replacement in patients with critical aortic stenosis: Rationale, device descriptions, early clinical experiences, and perspectives. Thoracic and Cardiovascular Surgery, v. 18, pp $165-174$. 
42. Levi, D.S., Raff, E., Stepan, L., Liu, J., Williams, R.J., Moore, J.W., Carman, G., 2005. Use of a covered stent modification to produce a transcatheter valve: laboratory and animal testing. ASAIO Journal, v. 51, no. 6, pp 719-724.

43. Laborde, J.C., Borenstein, N., Behr, L., Farah, B., Fajadet, J., 2005. Percutaneous implantation of an aortic valve prosthesis. Catheterization and Cardiovascular Interventions, v. 65, pp 171-174.

44. Lamarche, Y., Cartier, R., Denault, A.Y., Basmadjian, A., Berry, C., Laborde, J.C., Bonan, R., 2007. Implantation of the CoreValve Percutaneous aortic valve. The Annals of Thoracic Surgery, v. 83, no. 1, pp 284-287.

45. Fletcher, E.M., Curry, J.W., Joudinaud, T.M., Kegel, C.L., Weber, P.A., Duran, C.M.G., 2007. Coronary flow obstruction in percutaneous aortic valve replacement. An in vitro study. European Journal of Cardio-Thoracic Surgery, v.32, pp 291-295.

46. Feinstein, J.A., Kim, N, Reddy, V.M., Perry, S.B., 2006. Percutaneous pulmonary valve placement in a 10-month-old patient using a hand crafted stent-mounted porcine valve. Catheterization and Cardiovascular Interventions, v. 67, pp 644649.

47. Feldman, T. Percutaneous valve repair and replacement: challenges encountered, challenges met, challenges ahead. Circulation, v. 113, pp 771-773.

48. Davidson, M.J., White, J.K., Baim, D.S., 2006. Percutaneous therapies for valvular heart disease. Cardiovascular Pathology, v. 15, pp 123-129.

49. Vassiliades, T.A., Block, P.C., Cohn, L.H., Adams, D.H., Borer, J.S., Feldman, T., Holmes, D.R., Laskey, W.K., Lytle, B.W., Mack, M.J., Williams, D.O., 2005. The clinical development of percutaneous heart valve technology. Journal of the American College of Cardiology, v. 45, no. 9, pp 1554-1560.

50. Hanzel, G. S. and O'Neil, W. W. 2006. Complications of percutaneous aortic valve replacement: experience with the Cribier-Edwards percutaneous heart valve. EuroIntervention Supplements, v. 1, supp. A, pp A3-A8.

51. Berry, C., Cartier, R., Bonan, R., 2007. Fatal ischemic stroke related to nonpermissive peripheral artery access for percutaneous aortic valve replacement. Catheterization and Cardiovascular Interventions, v. 69, pp 56-63.

52. Webb, J.G., Pasupati, S., Achtem, L., Thompson, C.R., 2006. Rapid pacing to facilitate transcatheter prosthetic heart valve implantation. Catheterization and Cardiovascular Interventions, v. 68, pp 199-204. 
53. Nordmeyer, J., Coats, L., Bonhoeffer, P., 2006. Current experience with percutaneous pulmonary valve implantation. Seminars in Thoracic and Cardiovascular Surgery, v. 18, pp 122-125.

54. Nordmeyer, J., Khambadkone, S., Coats, L., Schievano, S., Lurz, P., Parenzan, G., Taylor, A.M., Lock, J.E., Bonhoeffer, P., 2007. Risk stratification, systematic classification, and anticipatory management strategies for stent fracture after percutaneous pulmonary valve implantation. Circulation, v. 115, no. 11, pp 13921397.

55. Paniagua, D., Induni, E., Ortiz, C., Mejia, C., Lopez-Jimenez, F., Fish, D., 2002. Percutaneous heart valve in the chronic in vitro testing model. Circulation, v. 106, pp 51-52.

56. Stock, U.A., Degenkolbe, I., Attmann, T., Schenke-Layland, K., Freitag, S., Lutter, G., 2006. Prevention of device-related tissue damage during percutaneous deployment of tissue-engineered heart valves, The Journal of Thoracic and Cardiovascular Surgery, v. 131, no. 6, pp 1323-1330.

57. Coats, L., Khambadkone, S., Derrick, G., Hughes, M., Jones, R., Mist, B., Pellrin, D., Marek, J., Deanfield, J.E., Bonhoeffer, P., Taylor, A.M., 2007. Physiological consequences of percutaneous pulmonary valve implantation: the different behavior of volume- and pressure-overloaded ventricles. European Heart Journal doi:10.1093/eurheartj/ehm181

58. Gallocher, S.L., Aguirre, A.F., Kasyanov, V., Pinchuk, L., Schoephoerster, R.T., 2006. A novel polymer for potential use in a trileaflet heart valve. Journal of Biomedical Materials Research Part B: Applied Biomaterials 79B: pp 325-334.

59. Wise, D.L., Trantolo, D.J., Lewandrowski, K-U., Gresser, J.D., Cattaneo, M.V., Yaszemski, M.J., 2000. Biomaterials Engineering and Devices Human Applications: Volume 1 Fundamentals and Vascular and Carrier Applications. Humana Press, Totowa, NJ.

60. Duerig, T.W., Tolomeo, D.E., Wholey, M., 2000. An overview of super-elastic stent design. Minimally Invasive Therapies and Allied Technologies, v. 9, no. 3/4, pp 235-246.

61. McKelvey, A.L. and Ritchie, R.O. 1999. Fatigue-crack propagation in nitinol, a shape memory and super-elastic endovascular stent material. Journal of Biomedical Materials, v.47, pp 301-308.

62. Stoeckel, D., Pelton, A., Duerig, T., 2004. Self-expanding nitinol stents: Material and design considerations. European Radiology, v. 14, pp 292-301. 
63. Zhou, S.S.N., How, T.V., Vallabhaneni, S.R., Gilling-Smith, G. L., Brennan, J. A., Harris, P.L., McWilliams, R., 2007. Comparison of the fixation strength of standard and fenestrated stent-grafts for endovascular abdominal aortic aneurysm repair. Journal of Endovascular Therapy, v. 14, pp 168-175.

64. Cribier, A., Eltchaninoff, H., Tron, C., Bauer, F., Agatiello, C., Nercolini, D., Tapiero, S., Litzler, P-Y., Bessou, J-P., Babaliaros, V., 2006. Treatment of calcific aortic stenosis with the percutaneous heart valve: Mid-term follow-up from the initial feasibility studies: The French experience. Journal of the American College of Cardiology, v. 47, no. 6, pp 1214-1223.

65. Cribier, A., Eltchaninoff, H., Tron, C., Bauer, F., Gerber, L., 2006. Percutaneous Implantation of aortic valve prosthesis in patients with calcific aortic stenosis: Technical advances, clinical results and future strategies. Journal of Interventional Cardiology, v. 19, no. 5, pp S87-S96.

66. Grube, E., Schuler, G., Buellesfeld, L. Gerckens, U., Linke, A., Wenaweser, P., Sauren, B., Mohr, F-W., Walther, T., Zickmann, B., Iversen, S., Felderhoff, T., Cartier, R., Bonan, R., 2007. Percutaneous aortic valve replacement for severe aortic stenosis in high-risk patients using second- and current third generation self-expanding CoreValve prosthesis. Device success and 30-day clinical outcome. Journal of the American College of Cardiology, v.50, no.1, pp 69-76.

67. Khambadkone, S., Coats, L., Taylor, A., Boudjemline, Y., Derrick, G., Tsang, V., Cooper, J., Muthurangu, V., Hedge, S.R., Razavi, R.S., Pellerin, D., Deanfield, J., Bonhoeffer, P., 2005. Percutaneous pulmonary valve implantation in humans: Results from 59 consecutive patients. Circulation, v. 112, pp 1189-1197.

68. Bonow, R. O., et al., 2006. ACC/AHA 2006 Guidelines for the Management of Patients with Valvular Heart Disease. Circulation, August 1, pp el-el48. (DOI: 10.1161/CirculationAHA.106.176857)

69. Arbeláez, F.J., 2005. THE DEVELOPMENT OF A CATHETER BASED HEART VALVE. A thesis submitted in partial fulfillment of the requirements for the degree of MASTER OF SCIENCE in BIOMEDICAL ENGINEERING, Biomedical Engineering Department, Florida International University, Miami, FL.

70. Webb, J.G., Chandavimol, M., Thompson, C.R., Ricci, D.R., Carere, R.G., Munt, B.I., Buller, C.E., Pasupati, S., Lichtenstein, S., 2006. Percutaneous aortic valve implantation retrograde from the femoral artery. Circulation, v. 113, no. 6, pp 842-50.

71. Knudsen, L.L., Andersen, H.R., Hasenkam, J.M., 1993. Catheter-implanted prosthetic heart valves. Transluminal catheter implantation of a new expandable 
artificial heart valve in the descending thoracic aorta in isolated vessels and closed chest pigs. The International Journal of Artificial Organs, v. 16, no. 5, pp 253262.

72. Boudjemline, Y. and Bonhoeffer, P, 2002. Steps toward percutaneous aortic valve replacement. Circulation, v.105, no.6, pp 775-778.

73. Boudjemline, Y., Bonnet, D., Sidi, D., Bonhoeffer, P., 2002. Percutaneous implantation of a biological valve in the aorta to treat aortic valve insufficiency-a sheep study. Medical Science Monitor, 8(4), BR113-116.

74. Zaharias, A., Cribier, A., and Eltchaninoff, H., 2007. Successful coronary intervention after percutaneous aortic valve replacement. Catheterization and Cardiovascular Interventions, v. 69, pp 522-524.

75. Gorlin, R. and Gorlin, S.G., 1951. Hydraulic formula for calculation of the area of the stenotic mitral valve, other cardiac valves, and central circulatory shunts. American Heart Journal, v. 41, no. 1, 1-29.

76. Khambadkone, S., Nordmeyer, J., Bonhoeffer, P., 2007. Percutaneous implantation of the pulmonary and aortic valves: indications and limitations. Journal of Cardiovascular Medicine, v, 8, pp 57-61.

77. Babaliaros, V., Cribier, A., Agatiello, C., 2006. Surgery insight: current advances in percutaneous valve replacement and repair. Nature Clinical Practice. Cardiovascular Medicine, v. 3, no. 5, 256-264.

78. Coats, L. and Bonhoeffer, P., 2007. New percutaneous treatments for valve disease. Heart, v. 93, no. 5, pp 639-644.

79. Block P.C. and Bonhoeffer, P., 2005. Percutaneous approaches to valvular heart disease. Current Cardiology Reports, v. 7, pp 108-113.

80. Green, J., Babaliaros, V., Block, P., 2007. Percutaneous aortic valve placement. An overview of catheter-based approaches. Cardiac Interventions Today, v. 1, no. 2 , pp 29-32, 40 .

81. Babaliaros, V. and Block, P., 2007. State of the art percutaneous intervention for the treatment of valvular heart disease: a review of the current technologies and ongoing research in the field of percutaneous valve replacement. Cardiology, v. 107, no. 2, pp 87-96.

82. Nordmeyer, J., Lee, T-Y., Bonhoeffer, P., 2006. Percutaneous pulmonary valve implantation: a 5-year projection. The American Heart Hospital Journal, v. 4, pp 205-206. 
83. Boudjemline, Y., and Bonhoeffer, P.. 2001. Percutaneous aortic valve replacement: will we get there? Heart, v. 86, no. 6, p 705

84. Bauer, F., Eltchaninoff, H., Tron, C., Lesault, P.F., Agatiello, C., Nercolini, D., Derumeaux, G., Cribier, A., 2004. Acute improvement in global and regional left ventricular systolic function after percutaneous heart valve implantation in patients with symptomatic aortic stenosis. Circulation, v. 110, pp 1473-1476.

85. Hara, H., Pedersen, W.R., Ladich, E., Mooney, M., Virmani, R., Nakamura, M., Feldman, T., Schwartz, R.S., 2007. Percutaneous balloon aortic valvuloplasty revisited: Time for a renaissance? Circulation, v. 115, pp e334-e338.

86. Schmidt, W., Andresen, R., Behrens, P., Schmitz, K-P., 2004. Comparison of mechanical properties of peripheral self-expanding nitinol and balloonexpandable stainless-steel stents. Electronic poster at the annual meeting and postgraduate course of the cardiovascular and interventional radiological society of Europe (CIRSE), Barcelona, Spain, 25.-29.9.2004.

87. Robertson, S.W. and Ritchie, R.O., 2007. In vitro fatigue-crack growth and fracture toughness behavior of thin-walled super-elastic nitinol tube for endovascular stents: a basis for defining the effect of crack-like defects. Biomaterials, v. 28, pp 700-709.

88. Robertson, S.W., Imbeni, V., Wenk, H-R., Ritchie, R.O., 2005. Crystallographic texture for tube and plate of the super-elastic/shape-memory alloy nitinol used for endovascular stents. J of Biomed Mater Res, v. 72A, pp 190-199

89. Stankiewicz, J.M., Robertson, S.W., Ritchie, R.O., 2007. Fatigue-crack growth properties of thin-walled super-elastic austenitic nitinol tube for endovascular stents. J Biomed Mater Res, v. 81A, pp 685-691.

90. Schievano, S., Petrini, L., Migliavacca, F., Coats, L., Nordmeyer, J., Lurz, P., Khambadkone, S., Taylor, A., Dubini, G., Bonhoeffer, P., 2007. Finite Element Analysis of Stent Deployment: Understanding Stent Fracture in Percutaneous Pulmonary Valve Implantation. Journal of Interventional Cardiology, v. 20, no. 6, pp 546-554.

91. ISO $5840: 2005(\mathrm{E})$

92. FDA Replacement Heart Valves Draft Guidance, V.5.0

93. Robaina, S., Jayachandran, B., He, Y., Frank, A., Moreno, M., Schoephoerster, R. T., Moore, Jr., J. E., 2003. Platelet Adhesion to simulated stented surfaces. The Journal of Endovascular Therapy, v. 10, pp. 978-986. 
94. Pinchuk, L., Wilson, G., Barry, J., Schoephoerster, R., Parel, J-M., Kennedy, J., 2008. Medical applications of poly(styrene-block-isobutylene-block-styrene) (“SIBS"). Biomaterials, v. 29, pp. 448-460.

13. APPENDICIES

MATLAB CODE

HYDRODYNAMICS CALCULATOR

$\%$ Vivitro Systems LHS Heart Valve Hemodynamics Parameter Calculation Program

$\%$ Written by Ted Claiborne July 2007

$\%$ Revised August 22, 2007

$\%$

clear all;

close all;

clc;

load-ASCII 703.txt; \% Load data into a matrix in the Workspace:

\% CHANGE FILE NAME FOR EACH NEW DATA FILE (ex: "123.txt")

ch $1=X 703(:, 1) ; \%$ Assign data from text file to variables and creat specific data arrays ch4 $=$ X703 (:4); \% ENTER FILE NAME HERE AS WELL, AFTER THE "X"

$\operatorname{ch} 3=X 703(:, 3)$;

Half HR $=70 / 2 \%$ ENTER HEART RATE NUMERICAL VALUE FOR DATA SET HERE BEFORE THE $" / "$

Target_CO $=3 \%$ ENTER TARGET CO NUMERICAL VALUE HERE

$A=\operatorname{size}(\operatorname{ch} 3) ; \%$ Find range of data array

$\mathrm{B}=\operatorname{size}(\mathrm{ch} 4)$;

$\mathrm{G}=\operatorname{size}(\operatorname{ch} 1)$;

$A=A(1) ; \%$ Define array starting point and value

$\mathrm{B}=\mathrm{B}(1)$;

$\mathrm{G}=\mathrm{G}(1)$;

$\mathrm{C}=$ zeros $(1, \mathrm{~A}) ; \%$ Set up data matrix for calculations

$\mathrm{D}=\mathrm{zeros}(1, \mathrm{~B})$;

$\mathrm{E}=\mathrm{zeros}(1, \mathrm{~A})$;

$\mathrm{H}=\mathrm{zeros}(1, \mathrm{G})$;

$i=1 ; \%$ Define counting variables 
$j=1$;

while $i<=B \%$ Create matrix of positive channel 4 values

while $(\mathrm{i}<=\mathrm{B}) \&(\mathrm{ch} 4(\mathrm{i})>0) \%$ Collect all positive values

$\mathrm{j}=\mathrm{j}+\mathrm{l}$;

$D(j)=\operatorname{ch} 4(i)$;

$\mathrm{i}=\mathrm{i}+\mathrm{1}$;

end

while $(\mathrm{i}<=\mathrm{B}) \&(\operatorname{ch} 4(\mathrm{i})<0) \%$ Ignore negative values $\mathrm{i}=\mathrm{i}+\mathrm{i}$;

end

end

$\mathrm{u}=1 ; \%$ define counting variables

$\mathrm{p}=0$;

while $\mathrm{u}<=\mathrm{G} \%$ Create a matrix of chl values

$$
\begin{aligned}
& p=p+1 ; \\
& H(p)=\operatorname{ch} 1(u) \\
& u=u+1
\end{aligned}
$$

end

ch1_max $=\max (\mathrm{H}) ; \%$ Determine $\max$ ch1 value

ch1_min $=\min (\mathrm{H}) ; \%$ Determine $\min \mathrm{ch} 1$ value

$\mathrm{MAP}=(\max (\mathrm{H})+2 * \min (\mathrm{H})) / 3 \%$ Calculate MAP from ch 1 matrix

sum_ch $4=\operatorname{sum}(\mathrm{D}) ; \%$ Calculate sum of D matrix

pos_mean_press_drop_ch $4=\operatorname{sum}(\mathrm{D}) / \mathrm{j} \% \mathrm{Calculate}$ and output channel 4 positive mean pressure drop $(\mathrm{mmHg})$

max press drop ch4=max(D) \%Find and output maximum channel 4 value (mmHg)

mean flow_rate $=$ mean $(\mathrm{ch} 3) \%$ Calculate and output $\mathrm{CO}$ from channel $3(\mathrm{~L} / \mathrm{min})$

$\mathrm{n}=1 ; \%$ Define counting variables

$\mathrm{m}=1$;

while $\mathrm{n}<=\mathrm{A} \%$ Create matrix with positive channel 3 values 
while $(\mathrm{n}<=\mathrm{A}) \&(\operatorname{ch} 3(\mathrm{n})>.5) \%$ Collect $\operatorname{ch} 3$ values above 0.5

$\mathrm{m}=\mathrm{m}+1$

$\mathrm{C}(\mathrm{m})=\operatorname{ch} 3(\mathrm{n})$;

$\mathrm{n}=\mathrm{n}+1$;

end

while $(\mathrm{n}<=\mathrm{A}) \&(\operatorname{ch} 3(\mathrm{n})<.5) \%$ Ignore ch 3 values below 0.5

$$
\mathrm{n}=\mathrm{n}+1
$$

end

end

stroke volume $=\left(\operatorname{trapz}(\mathrm{C}) * .005^{*}(1000 / 60)\right) /$ Half_HR $\%$ Calculate and output forward flow volume $(\mathrm{ml})$

sum_ch $3=\operatorname{sum}(\mathrm{C}) ; \%$ Calculate the sum of matrix $\mathrm{C}$

mean_forward_flow_rate $=\operatorname{sum}(\mathrm{C}) / \mathrm{m} \%$ Calculate the mean of matrix $\mathrm{C}$

$\mathrm{q}=1 ; \%$ Define new counting variables

$w=1$;

cardiac output $=$ stroke volume* Half $\mathrm{HR}^{*} 2 / 1000$

while $\mathrm{q}<=\mathrm{A} \%$ Create matrix of negative channel 3 values

while $(\mathrm{q}<=\mathrm{A}) \&(\operatorname{ch} 3(\mathrm{q})<(-.5)) \%$ Collect ch3 values below -0.5

$\mathrm{w}=\mathrm{w}+1$

$E(w)=\operatorname{ch} 3(q)$;

$\mathrm{q}=\mathrm{q}+1$;

end

while $(\mathrm{q}<=\mathrm{A}) \&(\operatorname{ch} 3(\mathrm{q})>(-.5)) \%$ Ignore ch3 values above -0.5

$q=q+1$

end

end

leakage_flow_volume $=\left(\operatorname{trapz}(\mathrm{E})^{*} .005 *(1000 / 60)\right) /$ Half_HR \% Calculate leakage flow volume ( $\mathrm{ml})$ using integration

$\mathrm{v}=1 ; \%$ Define new counting variables

$\mathrm{k}=1$; 
$\mathrm{F}=$ zeros $(1, \mathrm{~B}) ; \%$ Define new matrix

while $\mathrm{v}<=\mathrm{A} \%$ Create new matrix data set linking positive ch 3 values to $\mathrm{ch} 4$ values

while $(v<=A) \&(\operatorname{ch} 3(v)>.5) \%$ In order to obtain a better delta $P$ value we must ignore small oscillations around zero.

$\mathrm{k}=\mathrm{k}+1 ; \quad \%$ Collect $\mathrm{ch} 3$ values above 0.5

$F(k)=\operatorname{ch} 4(v)$;

$\mathrm{v}=\mathrm{v}+\mathrm{l}$ :

end

while $(\mathrm{v}<=\mathrm{A}) \&(\operatorname{ch} 3(\mathrm{v})<.5) \%$ Ignore ch 3 values below 0.5

$\mathrm{v}=\mathrm{v}+1$

end

end

ch4_sum $=\operatorname{sum}(\mathrm{F}) ; \%$ Calculate the sum of matrix $\mathrm{F}$

mean_press_drop_during_forward flow_ch $4=\operatorname{sum}(\mathrm{F}) / \mathrm{k} \%$ Calculate the pressure drop accross the valve during forward flow

Qrms $=$ norm $(\mathrm{C}) / \mathrm{sqrt}(\mathrm{m})$

effective_orifice area $=($ Qrms $*(1000 / 60)) /\left(51.6^{*}(\operatorname{sqrt}(\right.$ mean_press_drop_during_forward flow_ch4/1.133)))

$\%$

WINDKESSEL MODEL

ERROR FUNCTION

function model_error=error_fun $(\mathrm{x})$;

global yout tout Y R C;

$\%$ load experimental data

load TC707.txt;

$\%$ define variables

$\mathrm{Y}=\mathrm{TC} 707(1: 1001,1) ; \%$ pressure data matrix 
$\mathrm{R}=\mathrm{x}(1) ; \%$ compliance

$\mathrm{C}=\mathrm{x}(2) ; \%$ resistance

[tout,yout] $=$ sim('wind_model_ted',[0:1/200:5]);\%tout and yout generated by simulink model

length(Y);

length(yout);

model_error $=Y$-yout; \%define function

hist(model_error)

\section{OPTIMIZATION PROGRAM}

\%windkessel least squares curve fitting program using simulink model

clc;

clear all;

close all;

global yout tout Y R C; \%define global variables

wind_model_ted:

$\%$ load experimental data file

load TC707.txt;

$\%$ define variables

$\mathrm{Y}=\mathrm{TC} 707(1: 1001,1) ; \%$ pressure data

$\mathrm{x} 0=[.1 .1] ; \%$ initial guess for $\mathrm{R}$ and $\mathrm{C}$ values

$\mathrm{R}=\mathrm{x} 0(1) ; \%$ initial value for $\mathrm{R}$

$\mathrm{C}=\mathrm{x} 0(2) ; \%$ initial value for $\mathrm{C}$

$\mathrm{Po}_{\mathrm{O}}=\mathrm{Y}(1) ; \%$ initial condition for simulink integrator

$\mathrm{ub}=[2020] ; \%$ upper bound for estimates

$\mathrm{lb}=\left[\begin{array}{ll}0 & 0\end{array}\right] ; \%$ lower bound for estimates

[x,resnorm] $=$ lsqnonlin(@error_fun,x $0, \mathrm{lb}, \mathrm{ub}) \%$ least squares function

$\mathrm{R}=\mathrm{x}(1) * 60 / 1000$ 
$\mathrm{C}=\mathrm{x}(2)$

\%plot results

figure

plot(tout, Y, ', tout, yout);

xlabel('time (s)')

ylabel('pressure $\left.(\mathrm{mmHg})^{\prime}\right)$

legend('data', 'fit')

SOPS

SOP 01

1. Title: Fernando Jaramillo Arbeláez (FJA) Modified-Central Coaptation-Forward and Backward Hooks (MCB) Catheter Based Heart Valve (CBHV)

Manufacturing Process

2. Scope: This document is intended to describe the manufacturing process for the FJA-MCB percutaneous heart valve (PHV) design.

3. Author: Thomas E. Claiborne, III

4. Materials:

4.1 Small Parts, Inc. Super-Elastic Nitinol Wire 0.019 inch diameter by 36 inches long Part \# NW-019-36 (2 each)

4.2 Small Parts, Inc. Hypo Tube Thin-Walled 304 Stainless Steel 0.058 inch OD, 0.047 inch ID Part \# HTX-17T-36 (1 each)

4.3 PET woven fabric (Joanne Fabrics)

4.4 SIBS Polymer (Innovia, LLC)

4.5 Toluene Solvent

4.6 Glass jar

4.7 Stirring rod

4.8 Braided Polyester Suture (3-0 Ethibond Excel from Ethicon)

4.9 Aluminum Drying Plate

4.10 Water

4.11 Aluminum foil

4.12 Elmer's Krazy Glue

5. Equipment

5.1 Fume Hood

5.2 Stirring Plate

5.3 Furnace with temperature range to at least 600 degrees Celsius

5.4 Needle-Nose Pliers

5.5 Wire Cutters

5.6 Phillips and Flat-Head Screw Drivers

5.7 5-Gallon Bucket

5.8 Furnace Gloves

5.9 Furnace Tongs 
5.10 High speed rotary cutting tool

5.11 Eye protection

6. Nitinol Annealing Procedure

6.1 The Nitinol wire must be annealed in a two-step process to obtain the desired shape.

6.2 The Nitinol wire must be constrained in the first FJA wire forming mandrel.

6.3 Thread the Nitinol wire on the first mandrel as shown in figure 1 and constrain it with the end-plate and the wing-nut and washer.

6.4 While threading the Nitinol wire keep it taught by tensioning it with the needle-nose pliers while holding it in position on the mandrel with your hand.

6.5 The wire is very elastic and will try to spring off the mandrel if not constantly constrained.

6.6 Attach the constraining plates as shown in figure 2 .

6.7 Fill a 5 -gallon bucket about $2 / 3$ full with water and bring it to the furnace.

6.8 Locate furnace gloves and tongs and eye protection.

6.9 Place the mandrel as assembled in figure 2 into the furnace.

6.10 Set the furnace temperature to $500^{\circ} \mathrm{C}$.

6.11 Once the furnace has reached $500^{\circ} \mathrm{C}$ soak the assembly at that temperature for 5 minutes.

6.12 CAUTION! EXTREMLY HOT! After 2 minutes of soaking at or above $500^{\circ} \mathrm{C}$ CAREFULLY remove the mandrel using the tongs while wearing the furnace gloves.

6.13 Quench the mandrel in the water bucket immediately.

6.14 When the mandrel is cool to the touch, dry it off to prevent oxidation.

6.15 The Nitinol should look blue in color.

6.16 Remove the constraints and remove the Nitinol wire from the first mandrel.

6.17 Placed the Nitinol wire on the second mandrel as shown in figure 3.

6.18 Constrain the wire to form the hooks as shown in figure 4.

6.19 Repeat steps 6.9-6.16 (The furnace need not be cooled to room temperature for this step.)

6.20 The Nitinol wire should look like the one shown in figure 5.

6.21 Repeat steps to manufacture a second Nitinol wire. 


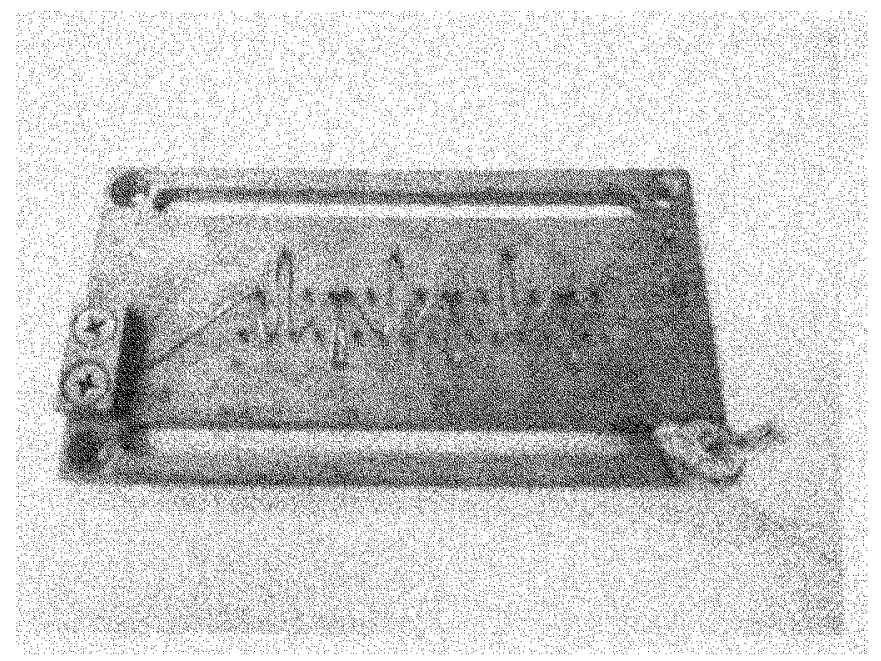

Figure 1: Threading pattern for Nitinol wire. Copper wire is shown here for illustrative purposes.

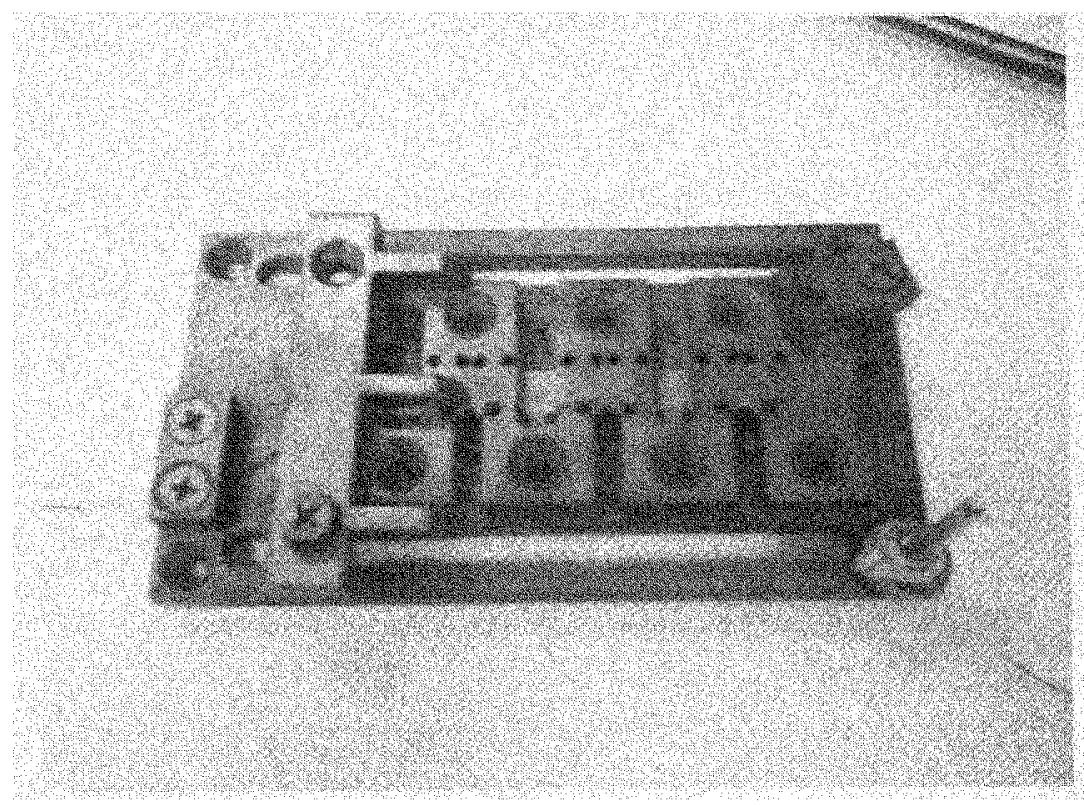

Figure 2: First mandrel with constraints. 


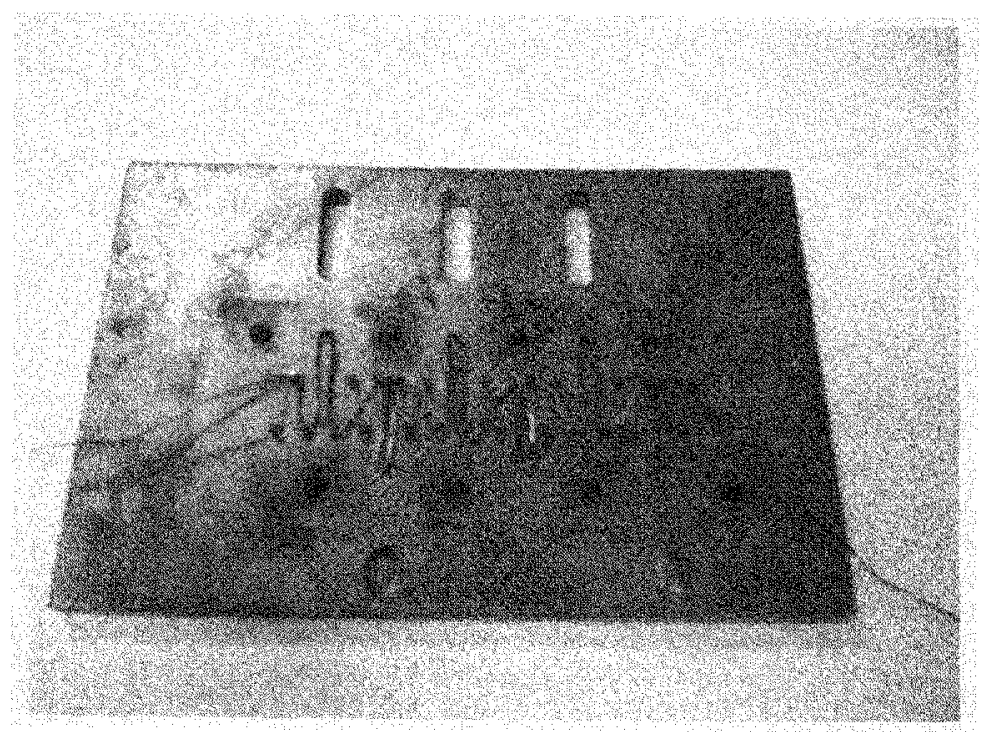

Figure 3: Second FJA mandrel. This is used to form the hooks of the MCB valve.

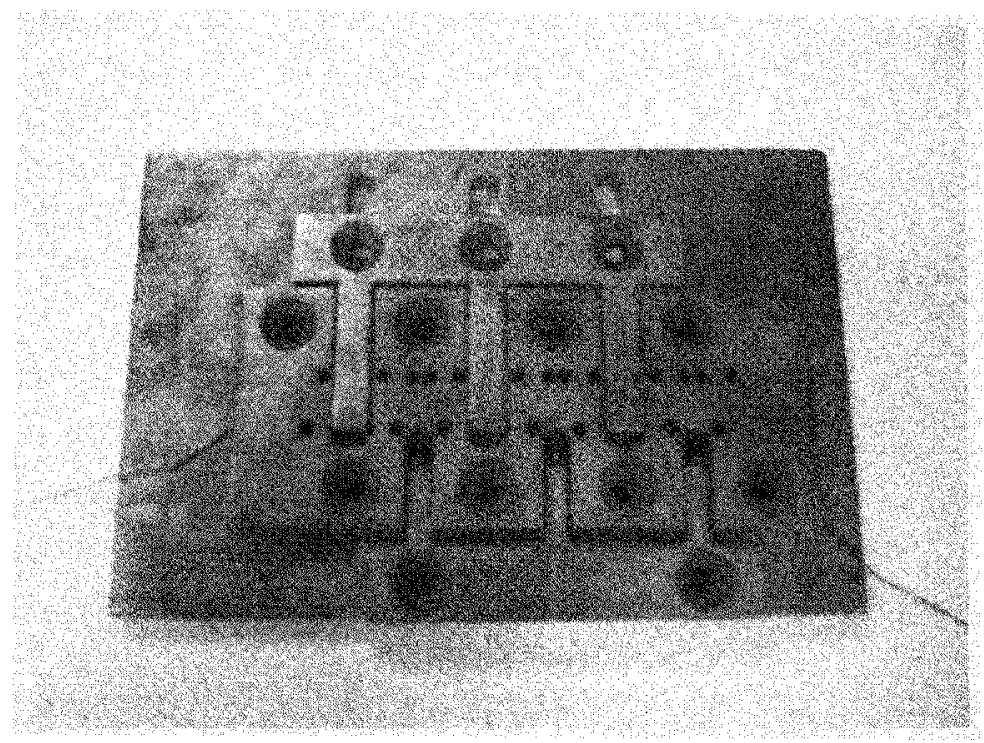

Figure 4: Second FJA mandrel with constraints. The bottom 3-fingered constraint plate lifts the wire loops off the mandrel at a certain angle, and the top 3-fingered constraint plate folds the wire loops over onto the center constraint plate. Place all screws (not shown). 


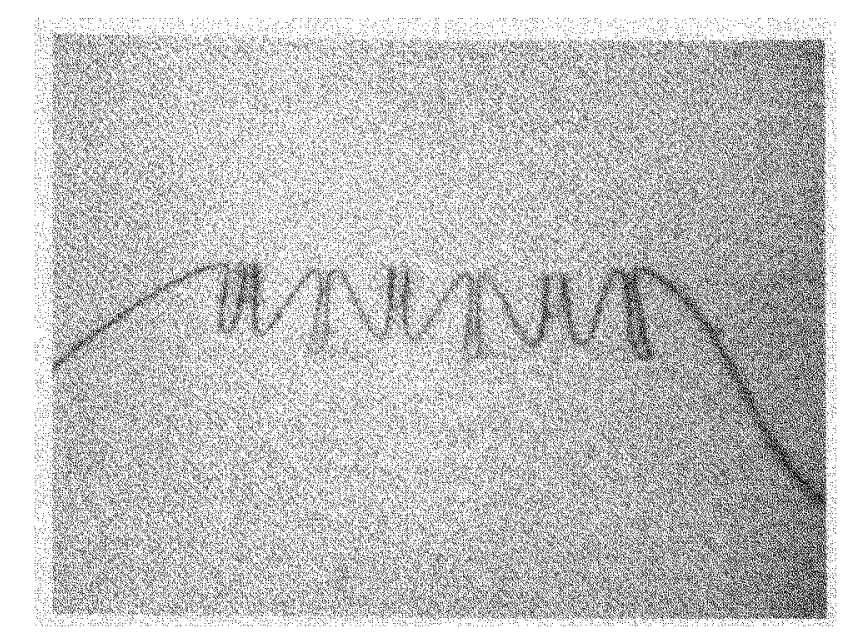

Figure 5: The final shape of the annealed Nitinol wire.

7. Stent Fabricating Procedure

7.1 Cut the Nitinol wire at each bent loop as shown in figure 6 .

7.2 Trim each cut so that a small hook is formed.

7.3 Cut 6 pieces of hypodermic tubing with the rotary cutting tool each about 1 $\mathrm{cm}$ long and use them to join the cut pieces of Nitinol wire. See figures 6 and 9 for reference. (Use eye protection)

7.4 Crimp each piece of hypo tubing and apply Elmer's Krazy Glue to the lumen of the hypo-tubing.

7.5 The final stent should be composed of $6 \mathrm{~N}$-shaped segments with alternating hooks.

8. Leaflet Fabrication Procedure

8.1 CAUTION! TOLUENE IS HIGHLY FLAMABLE AND NOXIOUS. THE FUME HOOD MUST BE UTILIZED.

8.2 Inside the fume hood, mix 5 grams of the SIBS polymer with toluene $15 \%$ by mass in a clean jar.

8.3 Place the jar onto the mixer with a stirring rod and leave until the SIBS is dissolved.

8.4 Affix the PET fabric to the drying plate (Figure 7).

8.5 Use aluminum foil as a gasket between the plate and the constraining lid.

8.6 Tightly constrain the fabric onto the drying plate.

8.7 Using a pipette, pour the SIBS solution onto the fabric in the drying plate.

Leave the assembly in the fume hood until it is dry.

8.8 Remove the fabric from the drying plate and cut it into three $25 \mathrm{~mm}$ strips.

8.9 Cut off the excess fabric.

8.10 Cut each strip into the shape shown in figure 8 . 


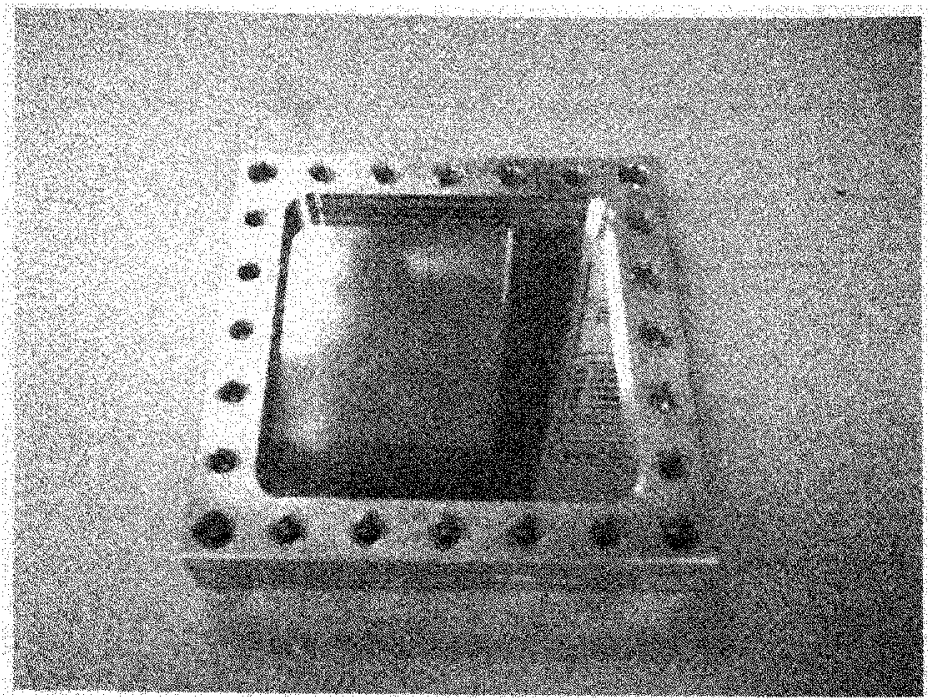

Figure 7: Drying plate.

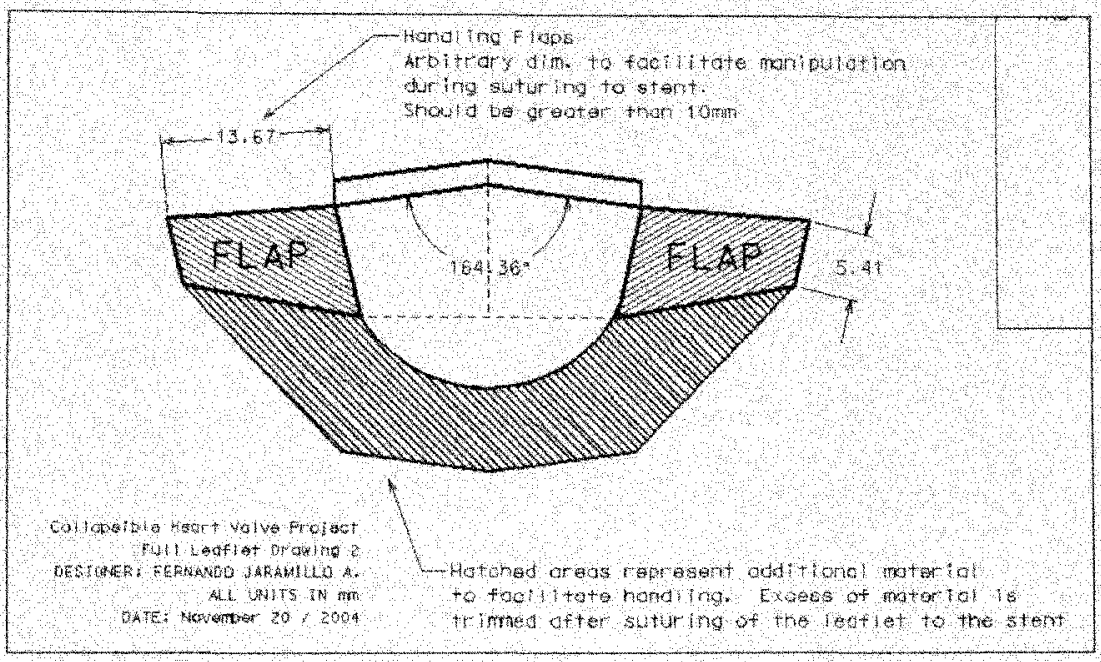

Figure 8: Pattern for MCB valve leaflet created by FJA.

9. Valve Assembly

9.1 Suture the leaflets onto the valve stent using the Ethibond suture.

9.2 Be sure to make a small cuff at the base of the valve.

9.3 The suture technique is subjective.

9.4 The final product is shown in figure 9 . 


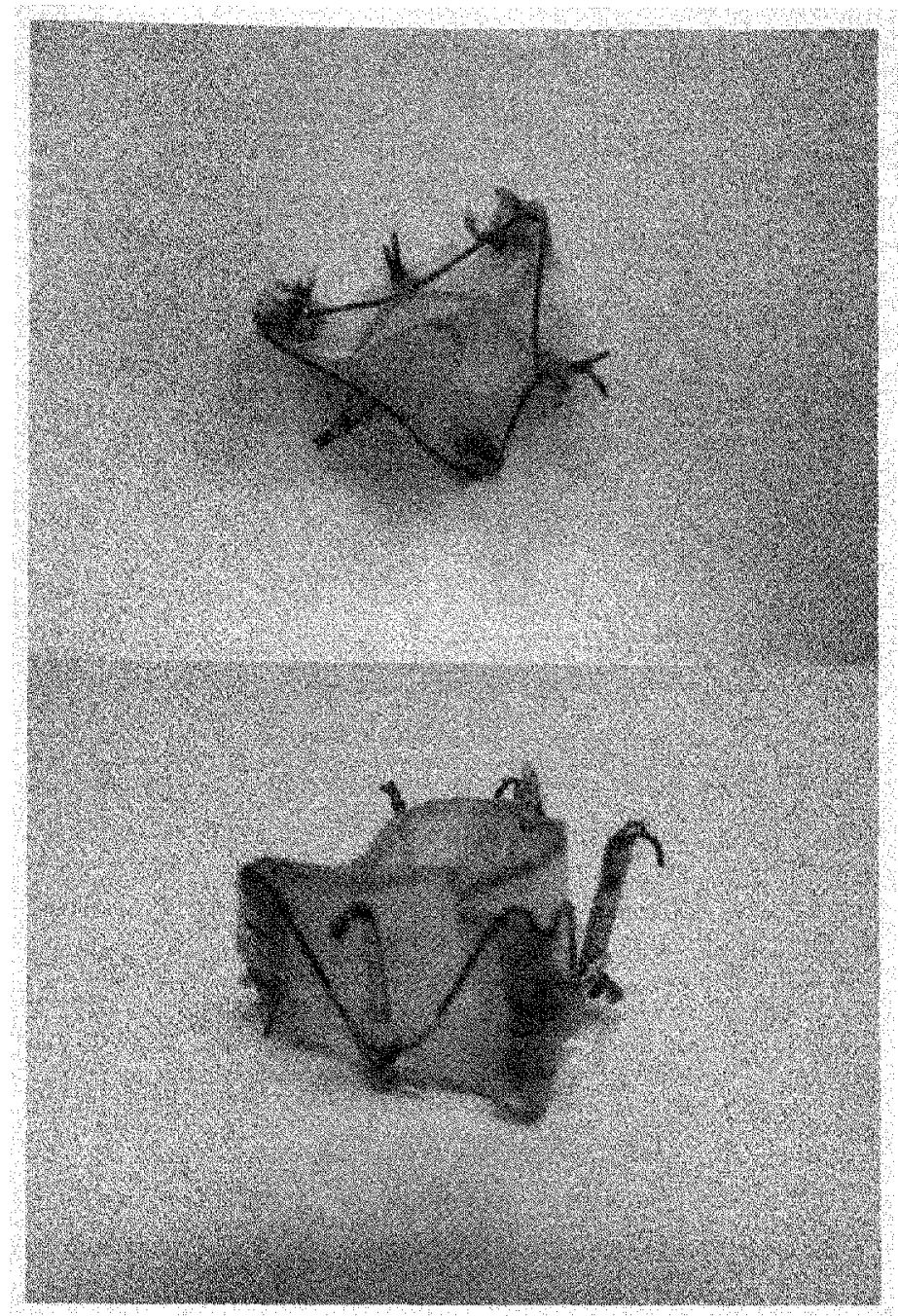

Figure 9: FJA MCB Valve.

SOP 02

1. Title: Vivitro Systems, Ine. Superdup'r Left Heart Simulator (LHS) Silicone Aorta (SA) Manufacturing Process

2. Scope: This document is intended to describe the manufacturing process for the author's design of an artificial aorta for use in the Vivitro LHS for delivery and hydrodynamic performance testing of percutaneous heart valves (PHV).

3. Author: Thomas E. Claiborne, III

4. Referenced Documents:

4.1 Solid Works Drawings by Claiborne of the SA Mold.

4.2 "Information About High Technology Silicone Materials: Sylgard 184 Silicone Elastomer, Base \& Curing Agent" by Dow Corning

5. Materials:

5.1 Dow Corning Sylgard 184 Silicone Elastomer Base 
5.2 Dow Corning Sylgard 184 Silicone Elastomer Curing Agent

5.3 Isopropyl Alcohol $70 \%$

6. Equipment

6.1 Low Temperature Oven

6.2 Vacuum Chamber

6.3 Scale

6.4 Weighing/Mixing Container

6.5 Stirring rod/Spatula

6.6 Paper Towels

6.7 Flat head screw driver

6.8 Hexagonal Wrenches

7. Procedure

7.1 Determine the ratio of curing agent to elastomer desired.

7.2 Determine silicone tube the wall thickness desired.

7.3 Make sure the mold is clean and dry (Figure 1).

7.4 Put the two halves of the mold together by aligning the dowel pins (Figure 2).

7.5 Insert the 4 larger screws, but do not completely tighten yet.

7.6 Measure 50-60 grams of Sylgard 184 Silicone Elastomer Base in a mixing container.

7.7 Add 5-6 grams of Sylgard 184 Silicone Elastomer Curing Agent for a standard 10:1 ratio as recommended by the manufacturer. Figure 3 shows the scale used.

7.8 Stir mixture until thoroughly mixed.

7.9 Place the mixing container into the vacuum chamber and set to $15-20 \mathrm{in} \mathrm{Hg}$. Leave until all air is removed from the mixture.

7.10 Pour the air-free Sylgard mixture into the mold.

7.11 Slowly insert the mold spacer/pin into the mold until the top is flush with the mold.

7.12 Wipe off all overflowing Sylgard.

7.13 Insert the spacer screws.

7.14 Tighten all screws.

7.15 Place the mold in a location where it will not be disturbed.

7.16 Allow the Sylgard to cure at room temperature for 24-48 hours.

7.17 When the polymer has dried, remove all screws and open the mold.

7.18 Using alcohol as a release agent aids this process.

7.19 Carefully pry the mold halves apart. Gently work the halves apart using a

flat head screw driver if necessary. (Aluminum is soft so vigorous prying will scar the surface.)

7.20 Pull the molded part and spacer assembly out of the mold clam shell.

7.21 Use a spatula to make a small gap between the silicone part and the spacer.

7.22 Bathe the spacer and silicone part with alcohol. The alcohol will facilitate easy removal of the silicone part from the spacer. Gently work the alcohol into a small space between the spacer and the silicone part. 
7.23 Gently pull the silicone part from the spacer. Figure 5 shows the final molded silicone part.

7.24 Figure 7 shows an alternate mold configuration using a spacer designed to make a thinner walled silicone aorta.

7.25 Clean and dry the mold assembly. Figure 6 shows the mold with the silicone part.

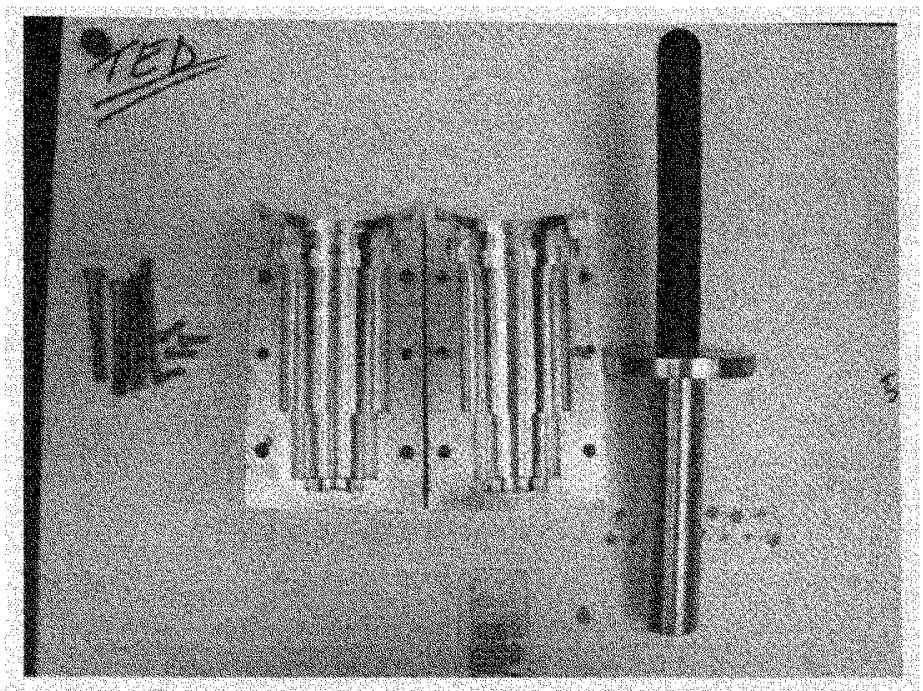

Figure 1: Vivitro aorta mold machined from 6016 Aluminum. Two halves joined by two steel dowel pins and 4 socket head hex-screws. The mold insert has a black Delrin handle that is removable. The mold insert is secured with 4 flat head hex-screws. All screws are shown on the left.

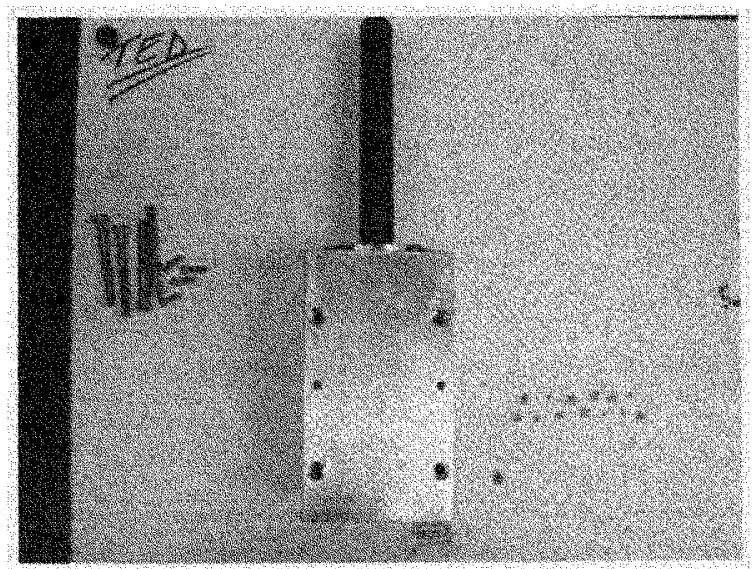

Figure 2: Assembled mold with insert. The screws have not been placed in the mold in this photograph. The spacer insert is not fully engaged into the mold. This image is for general illustrative purposes. The mold assembly process is very simple and intuitive. 


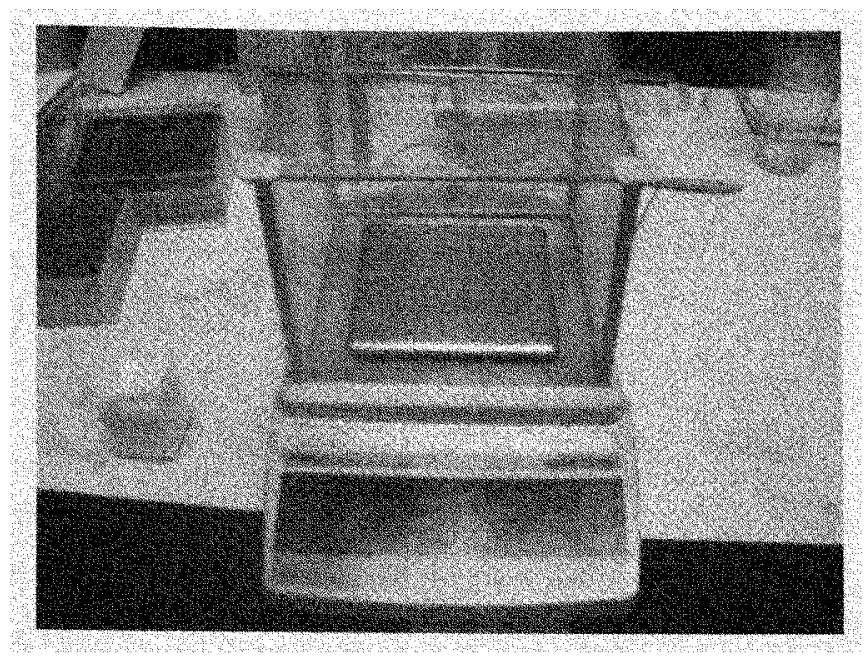

Figure 3: Mettler Toledo digital scale.

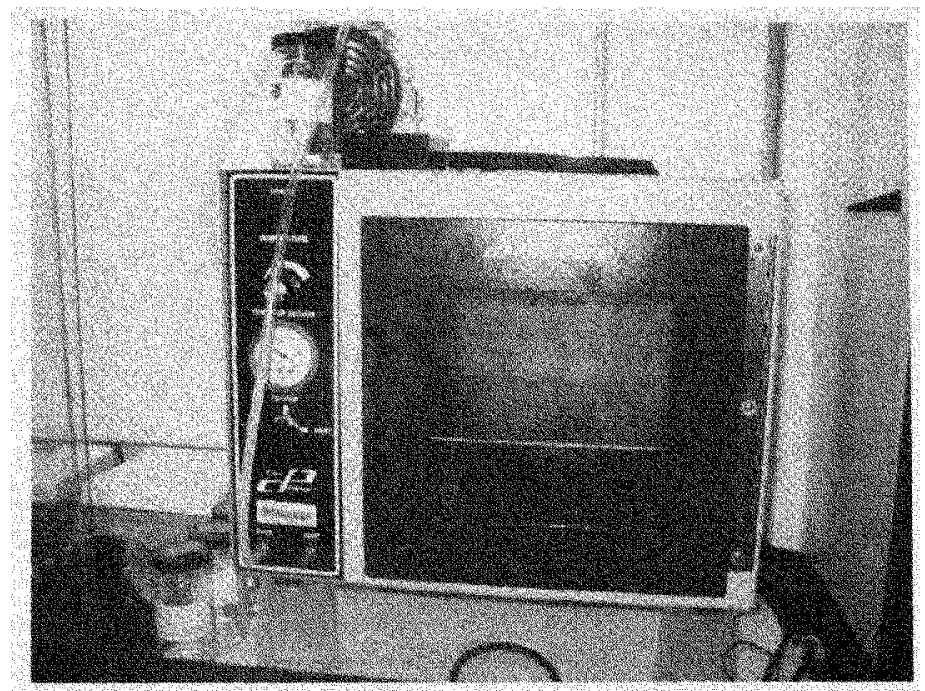

Figure 4: Cole Parmer Vacuum Chamber. 


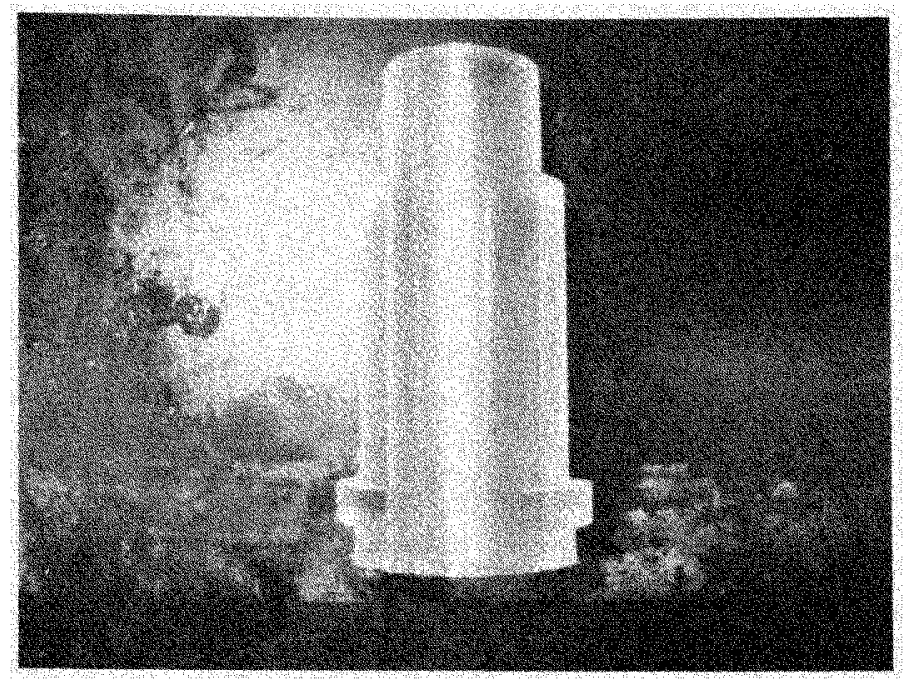

Figure 5: Silicone elastomer "Aorta". The part should be virtually free of air bubbles.

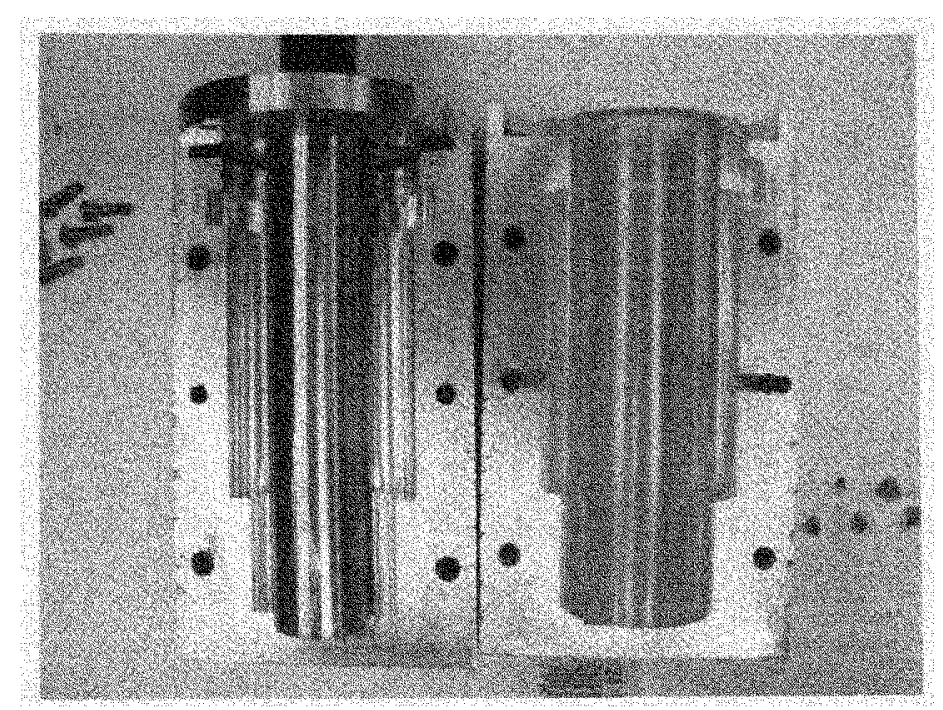

Figure 6: Mold with silicone part and insert. This image is for illustrative purposes only. 


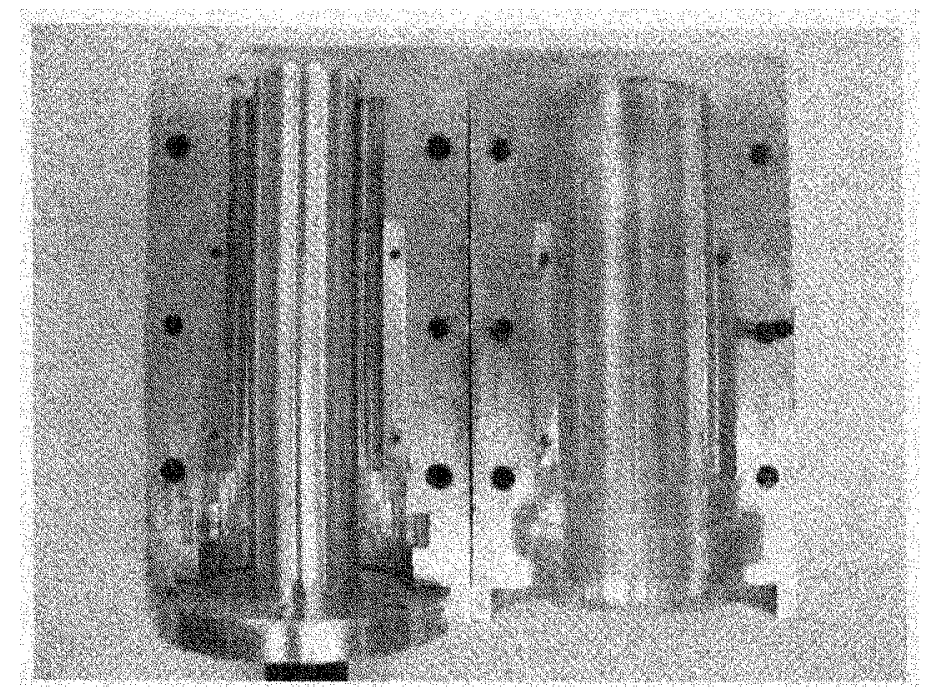

Figure 7: Mold with spacer and resulting silicone aorta.

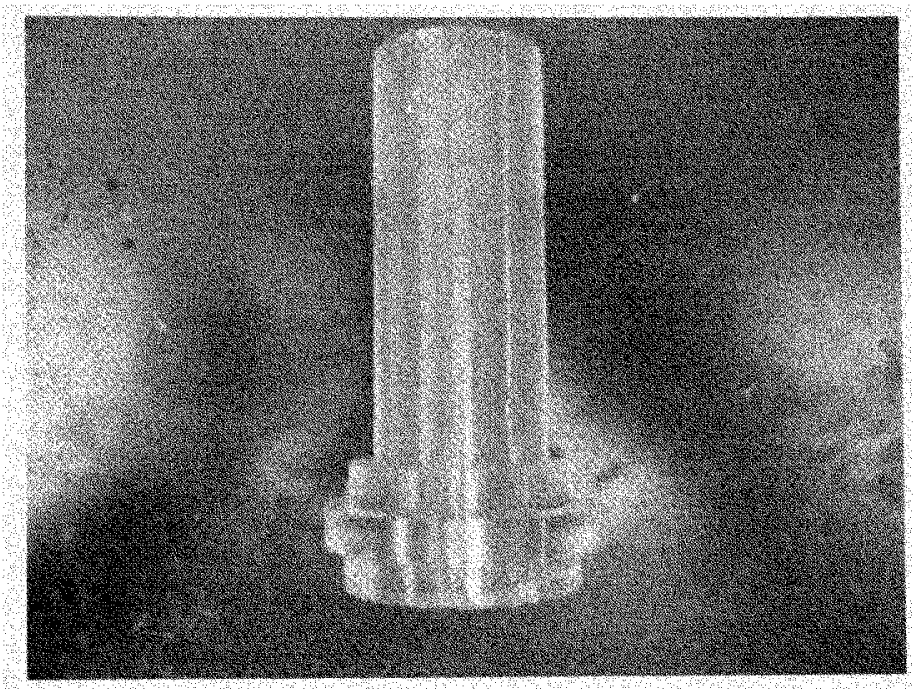

Figure 8: Thinner walled silicone aorta. 


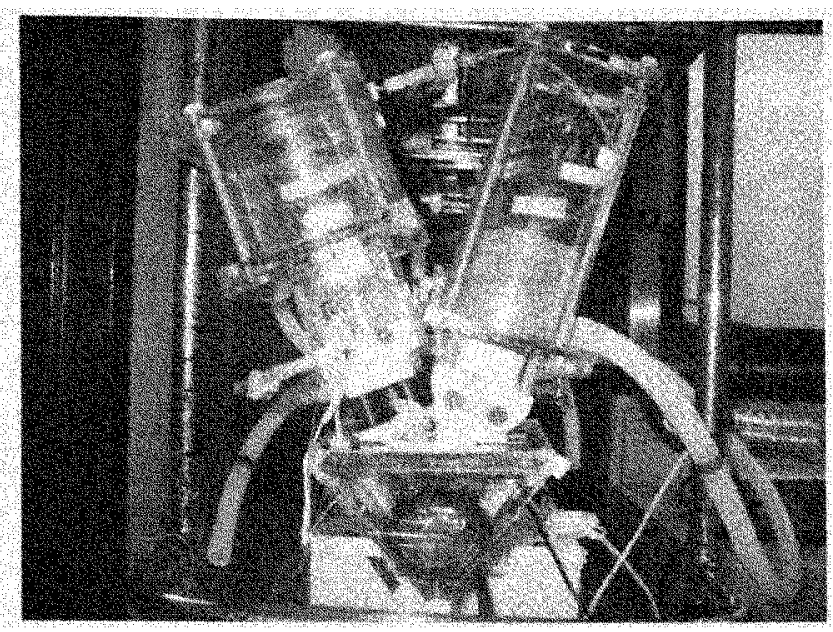

Figure 9: Vivitro LHS shown for reference.

SOP 03

1. Title: Vivitro Systems, Inc. Superdup'r Left Heart Simulator (LHS) Valve Test Data Analysis Process

2. Scope: This document is intended to describe the data analysis process for the purpose of creating reports that contain relevant hydrodynamic parameters for the evaluation of heart valve prosthesis performance.

3. Author: Thomas E. Claiborne, III

4. Referenced Documents:

4.1 None

5. Materials:

5.1 Printer paper

5.2 Printer ink

6. Equipment

6.1 PC

6.2 MatLab Software 6 or later

6.3 Acqknowledge Software 3.7.3

6.4 Microsoft Excel Spreadsheet Software 2003 or later

6.5 Printer

7. Acqknowledge Procedure

7.1 Open Acqknowledge 3.7.3

7.2 Open your baseline data file.

7.3 Select the I-bar at the bottom right of the screen as shown in figure 1 .

7.4 Go to Edit $\rightarrow$ Select All as shown in figure 1.

7.5 Record the values shown in Channels 1 to 3 . These are the " $\mathrm{K}$ " values that will be used to transform the waveforms in all channels. $\mathrm{K}$ refers to a constant which amounts to a correction factor that effectively zeros all of your data.

7.6 Close the baseline data file.

7.7 Open a test data file. 
7.8 Select Transform $\rightarrow$ Waveform Math as shown in figure 2.

7.9 A window called Waveform Arithmetic will open as shown in figure 3.

7.10 Select CH 1 under Source 1, select (-) under Operand, select K under

Source 2, and select CH 1 under Destination. Enter your K-value for channel

1 where it reads "Constant=."

7.11 Check the box labeled "Transform entire waveform."

7.12 Click OK.

7.13 Now you have transformed channel 1.

7.14 Repeat steps for channels 2 and 3 using the related K-values.

7.15 Now you need to create a new waveform in channel 4 that will show the pressure gradient across the test valve.

7.16 Again select Transform $\rightarrow$ Waveform Math as shown in figure 2 .

7.17 The Waveform Arithmetic window will again open as shown in figure 3.

7.18 This time select $\mathrm{CH} 2$ under Source 1, select (-) under Operand, select CH 1 under Source 2, select New under Destination, and check the box labeled

"Transform entire waveform" as shown in figure 4.

7.19 Now your will see channel 4.

7.20 Go to Display $\rightarrow$ Autoscale waveforms to adjust the view.

7.21 In the channel read-out bar select Channel 4 as shown in figure 5 .

7.22 Under channel 4 select mean as shown in figure 6.

7.23 Select File $\rightarrow$ Save.

7.24 Then select File $\rightarrow$ Save As... and save the file as a .txt file with a numeric name.

7.25 Repeat steps for each test data file. 


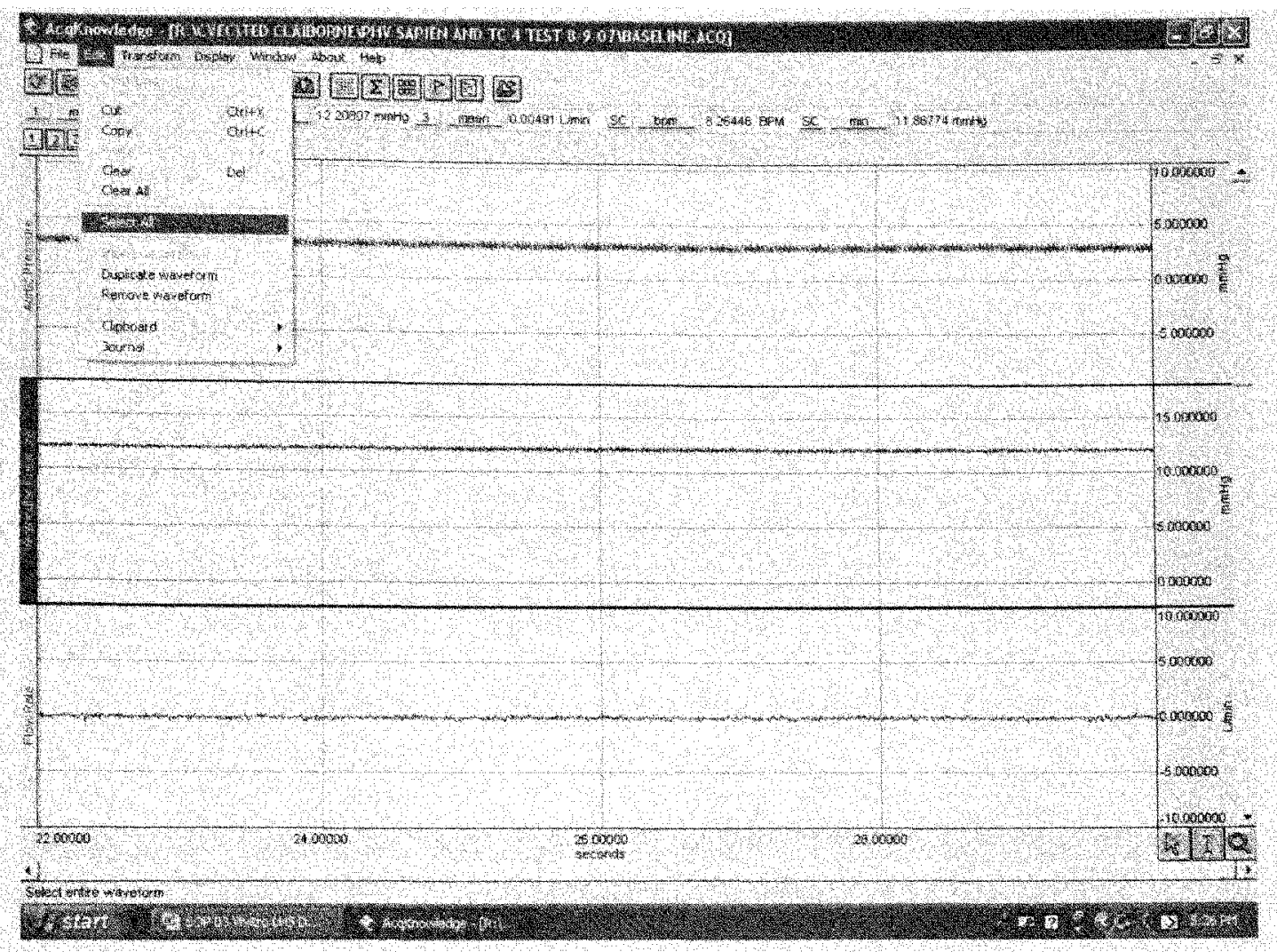

Figure 1: Screenshot of baseline data file showing where to find Edit functions. 


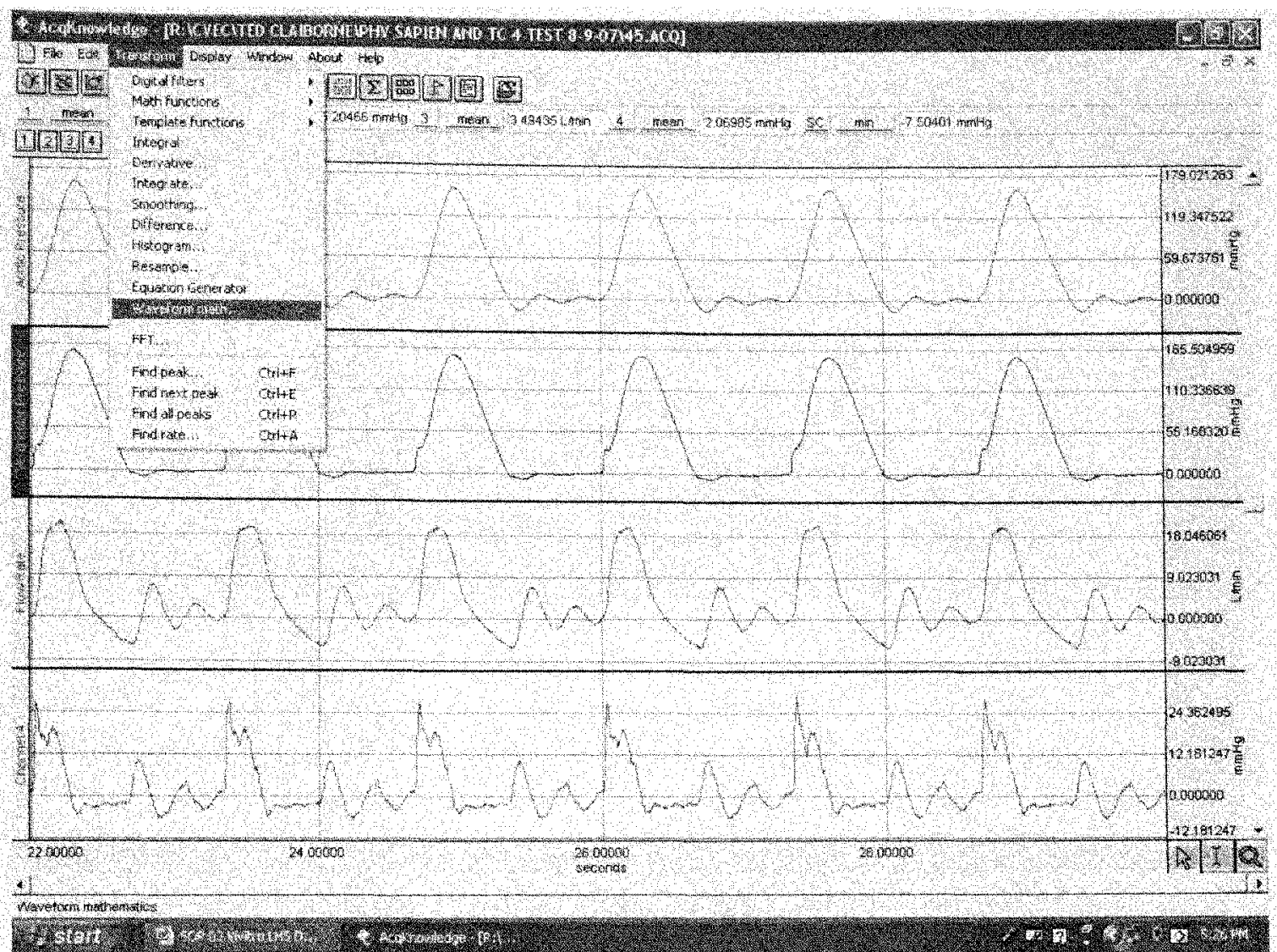

Figure 2: Screenshot of test data file showing where to find the waveform math function. 


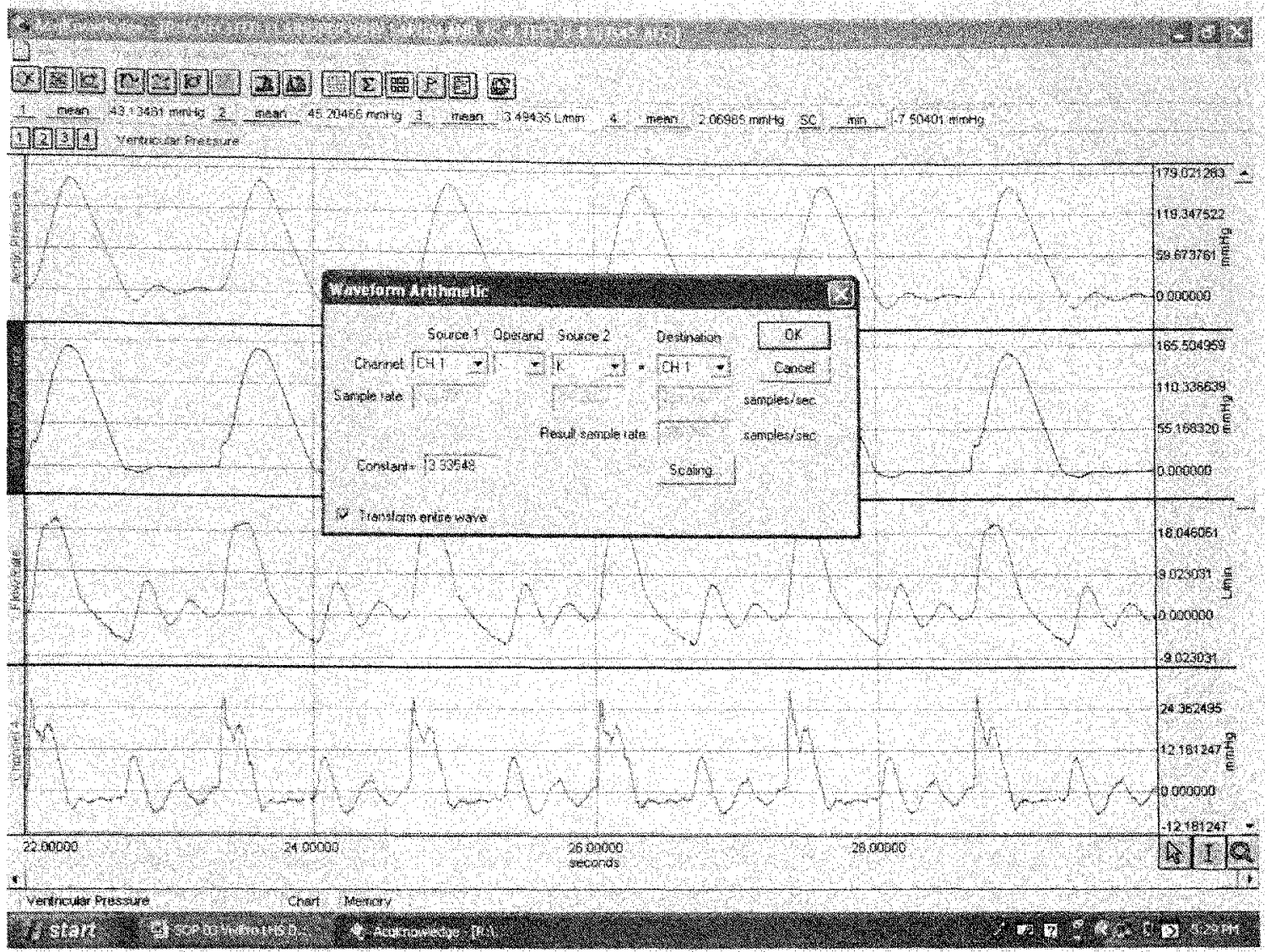

Figure 3: Screenshot showing the waveform arithmetic window. 


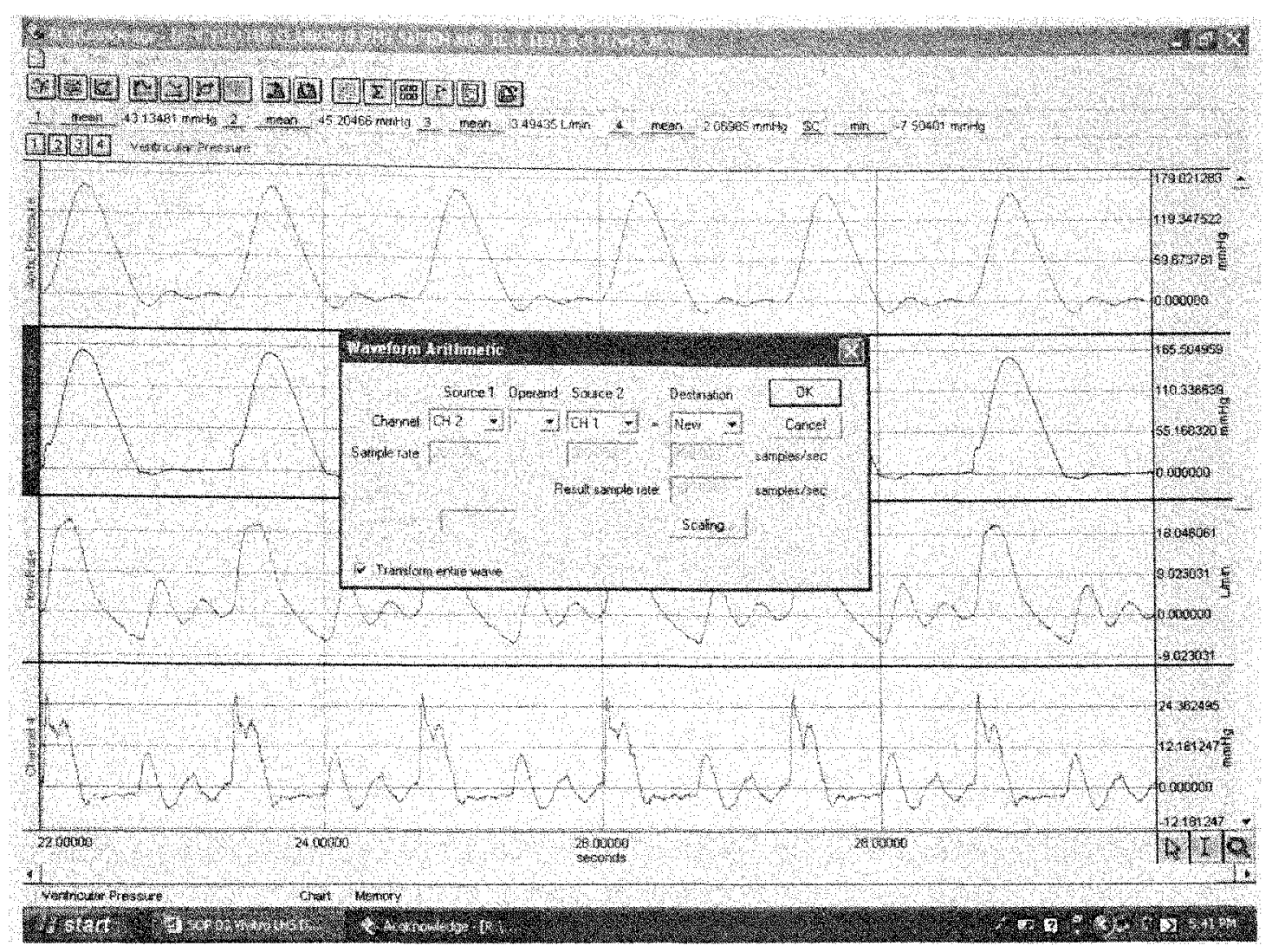

Figure 4: Screenshot of Waveform Arithmetic showing which values to input. 


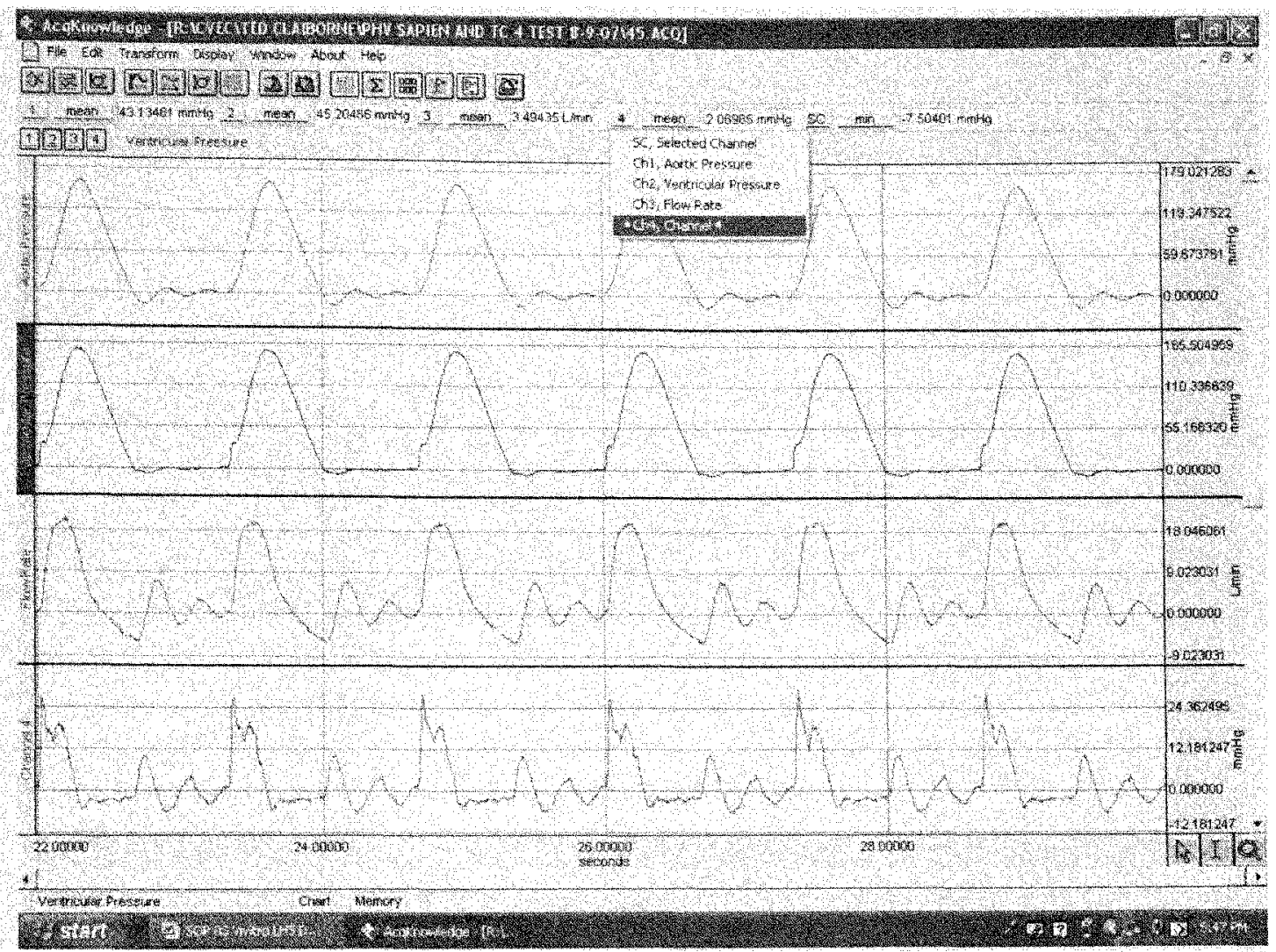

Figure 5: Screenshot showing where to find Channel 4 selection. 


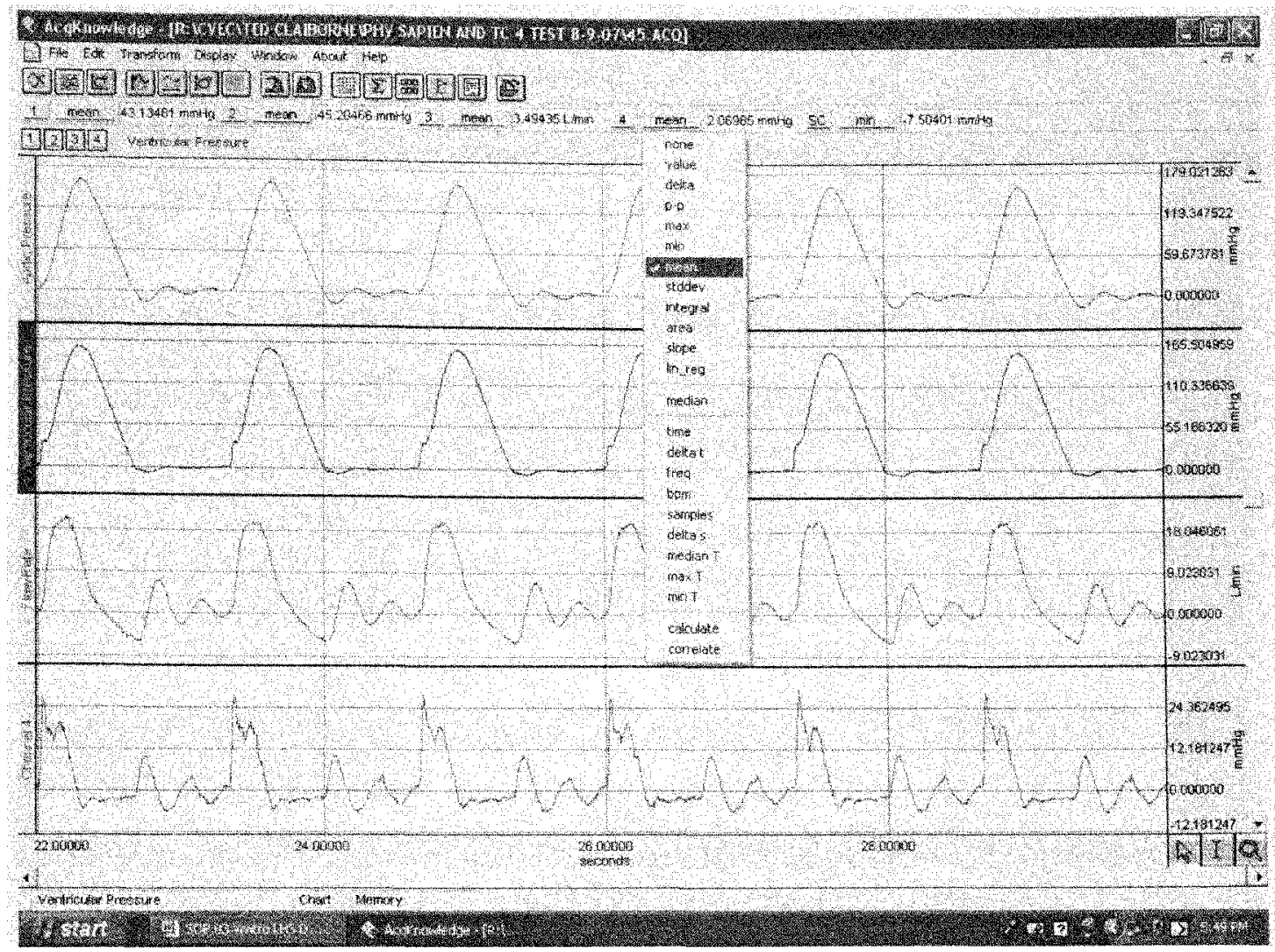

Figure 6: Screenshot showing where to find mean for channel 4.

8. Matlab Procedure

8.1 Create a New Folder on the hard drive.

8.2 Place all of your transformed txt data files into the new folder.

8.3 Copy your .m Matlab program into the new folder.

8.4 Open Matlab 6 or later.

8.5 Go to the Current Directory window and click the browse for folder button as shown in figure 7.

8.6 Browse for your newly created folder and select it in the Browse for Folder window as shown in figure 8.

8.7 Click OK.

8.8 Now all of your data files and the. $\mathrm{m}$ file are in the Current Directory as shown in figure 9.

8.9 Now double-click your .m file. If you are using the $m$ file written by Ted Claiborne then continue.

8.10 Follow the instructions written next to the code as shown in figure 10.

8.11 Once you have changed the file names and the heart rate in the $\mathrm{m}$ file click Run or press F5 on the key board.

8.12 The data used for calculation is now in your Workspace and the output information is now in your Command Window as shown in figure 11.

8.13 Select the command window. 
8.14 Select File $\rightarrow$ Print $\rightarrow$ OK.

8.15 Repeat steps for each data set.

8.16 Compile all data from the print-outs into an Excel file.

8.17 Create plots of Pressure Drop during Forward Flow as a function of Mean Forward Flow and Percent Regurgitation as function of Cardiac Output.

8.18 End.

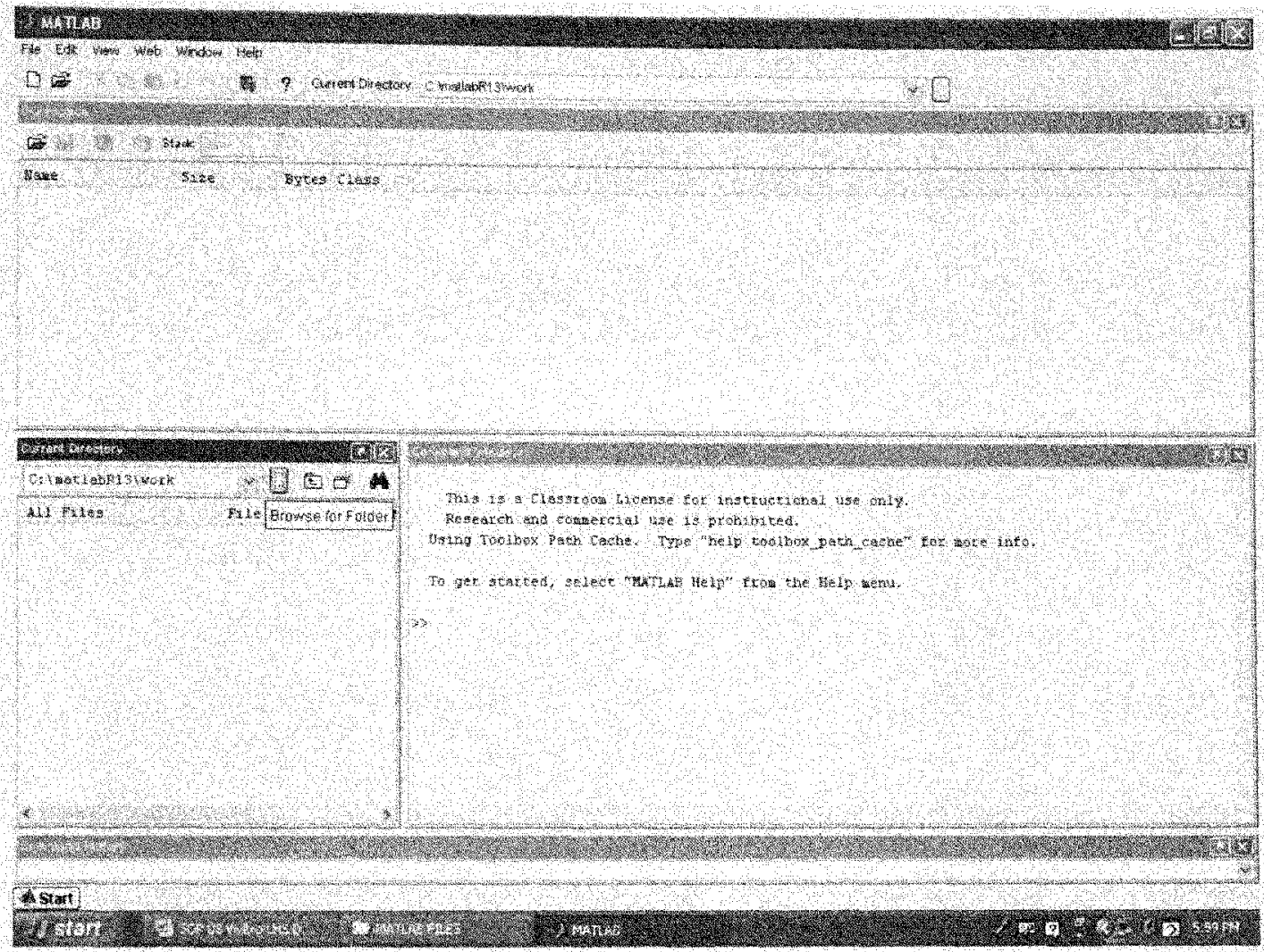

Figure 7: Screenshot showing where to map to a working directory. 


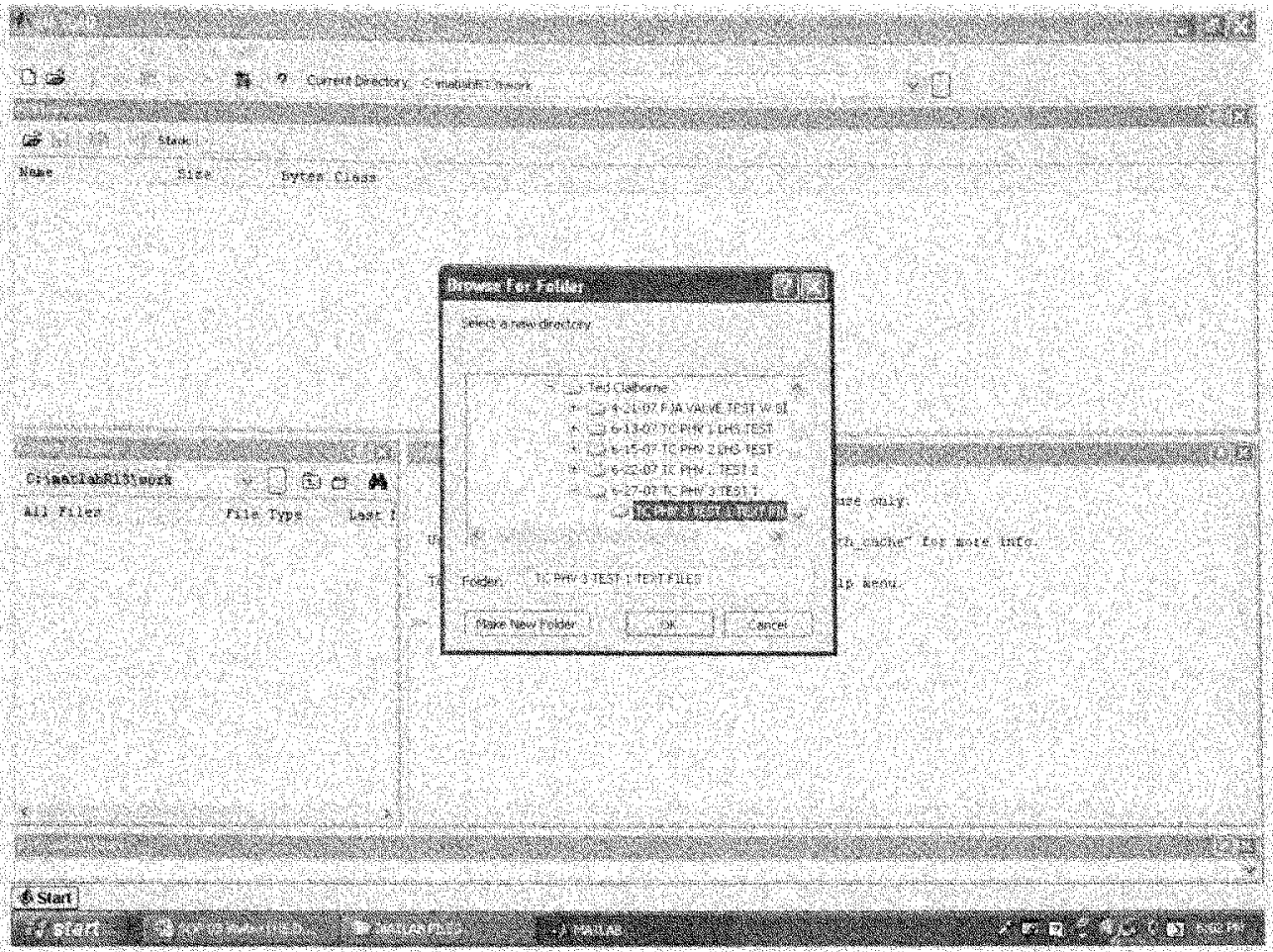

Figure 8: Screenshot showing where to find your working folder.

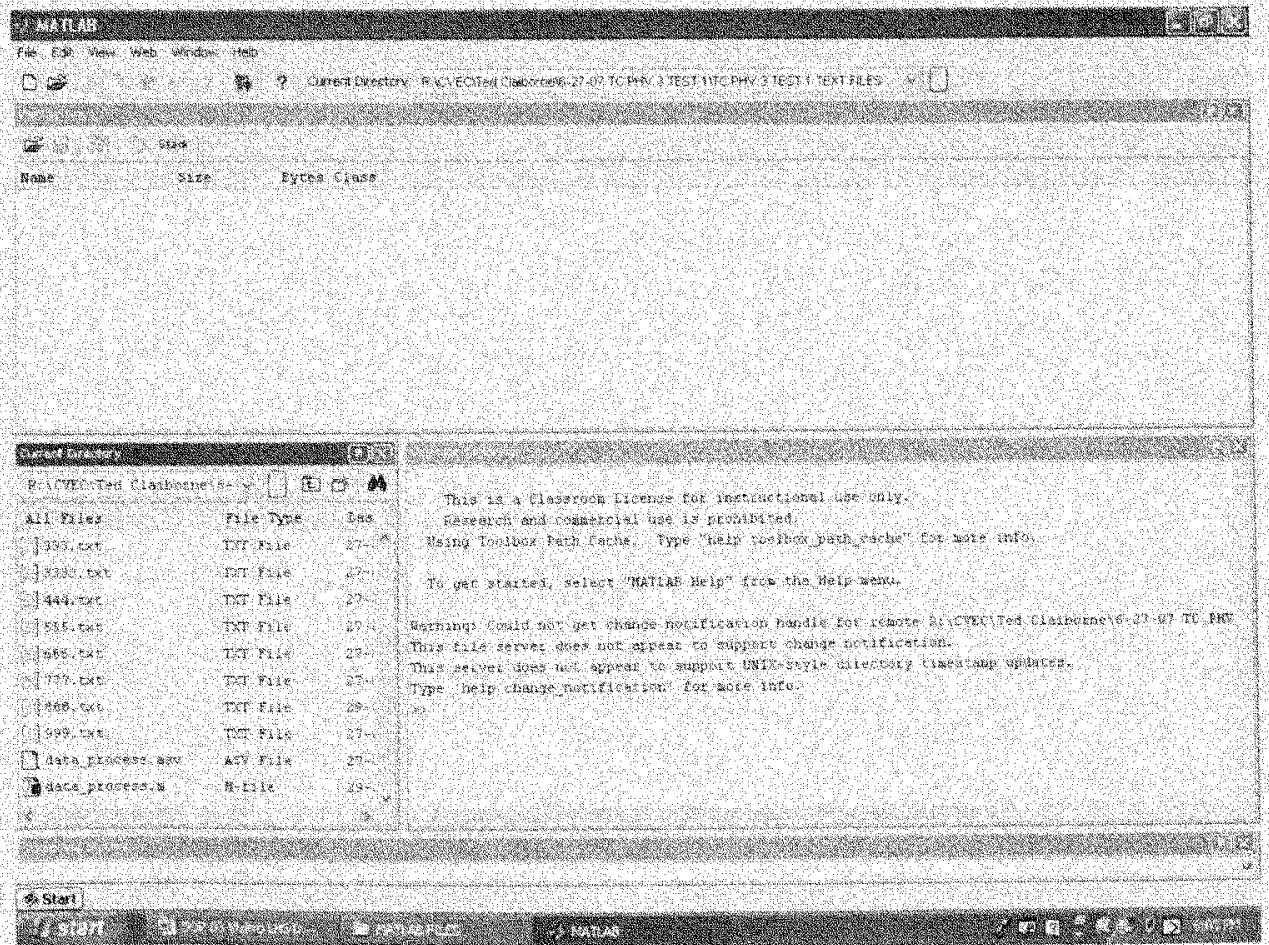

Figure 9: Screenshot of current directory. 


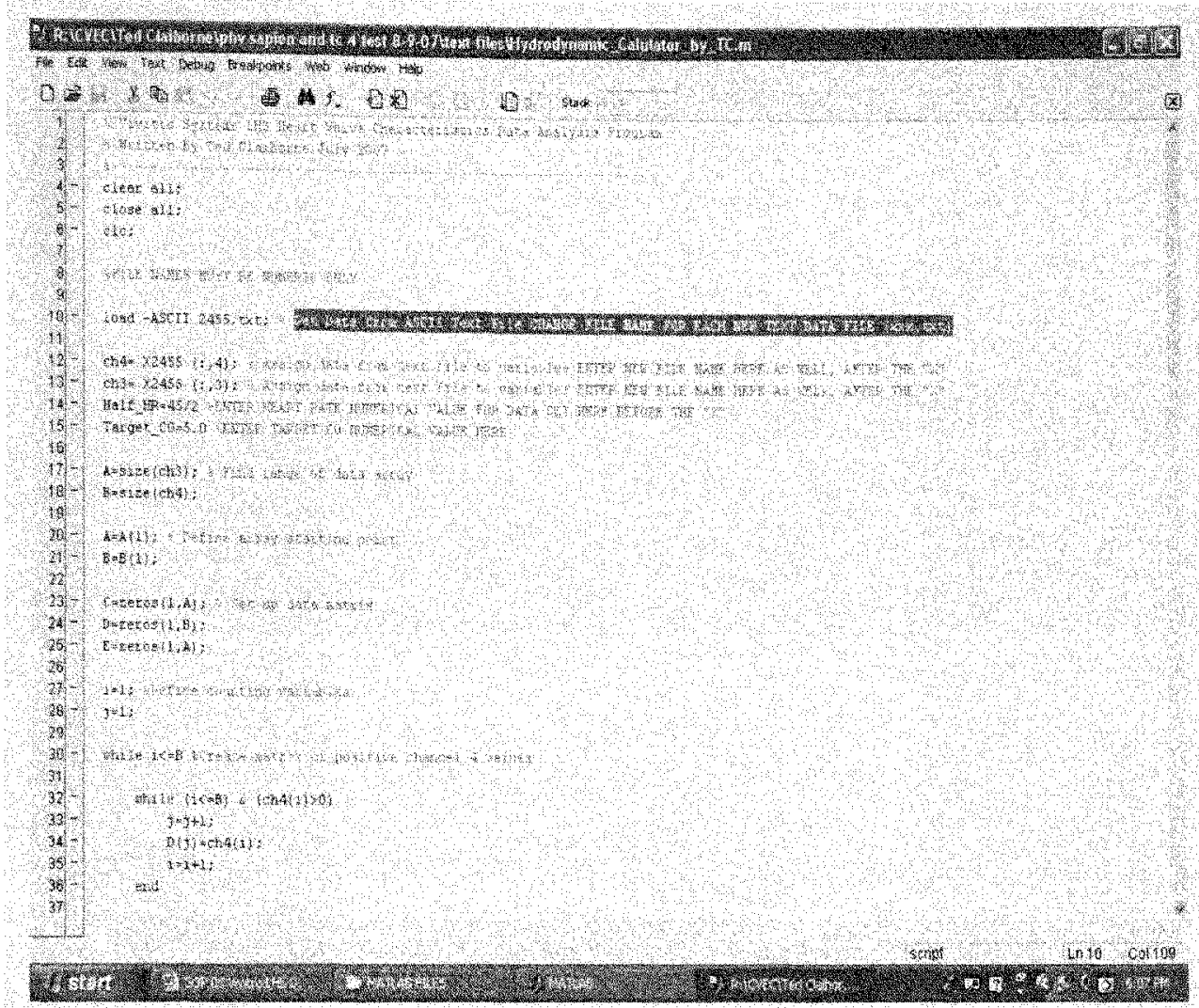

Figure 10: Screenshot of Ted Claiborne's .m file with instructions highlighted. 


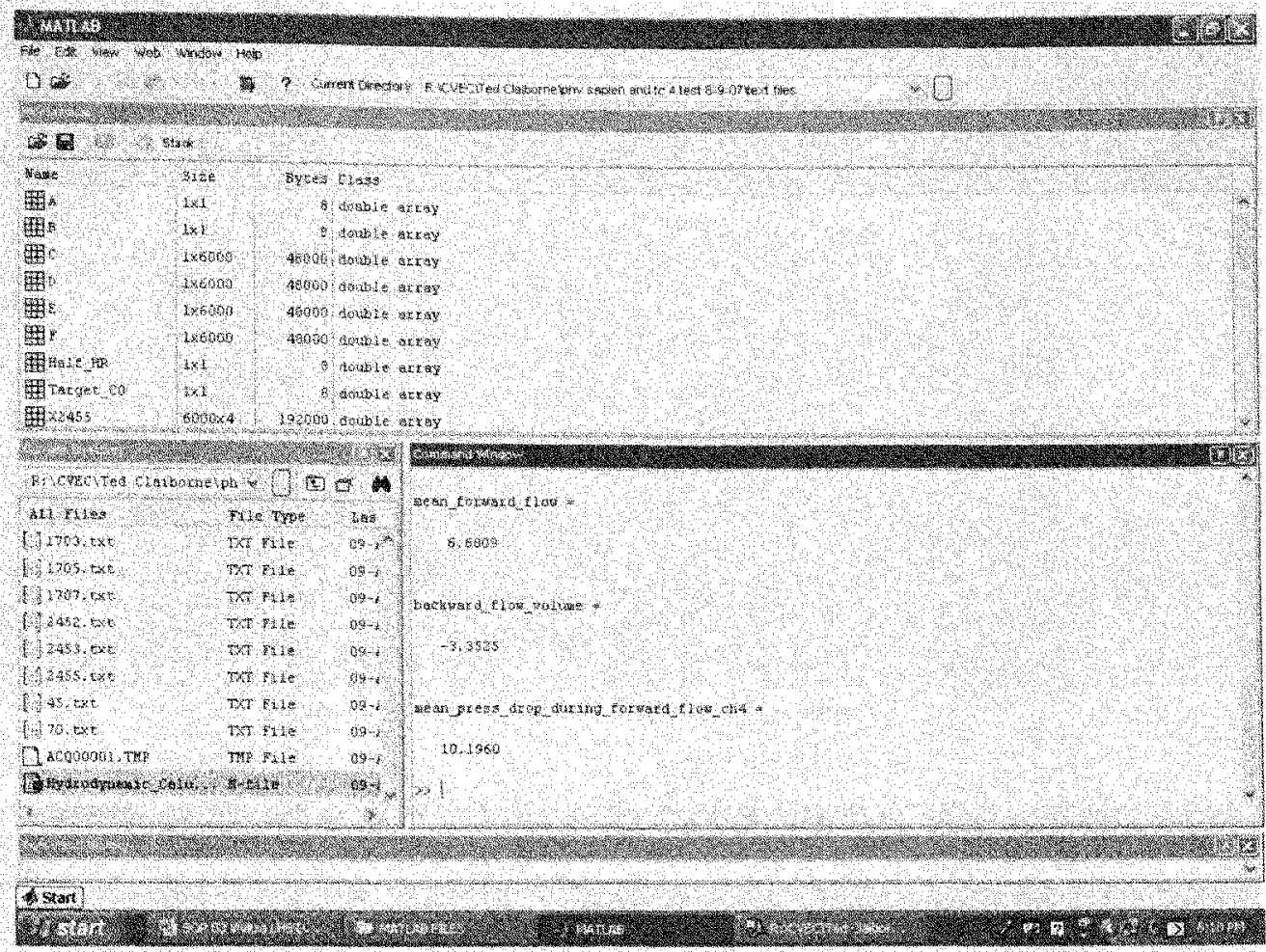

Figure 11: Screenshot of data and program output. 


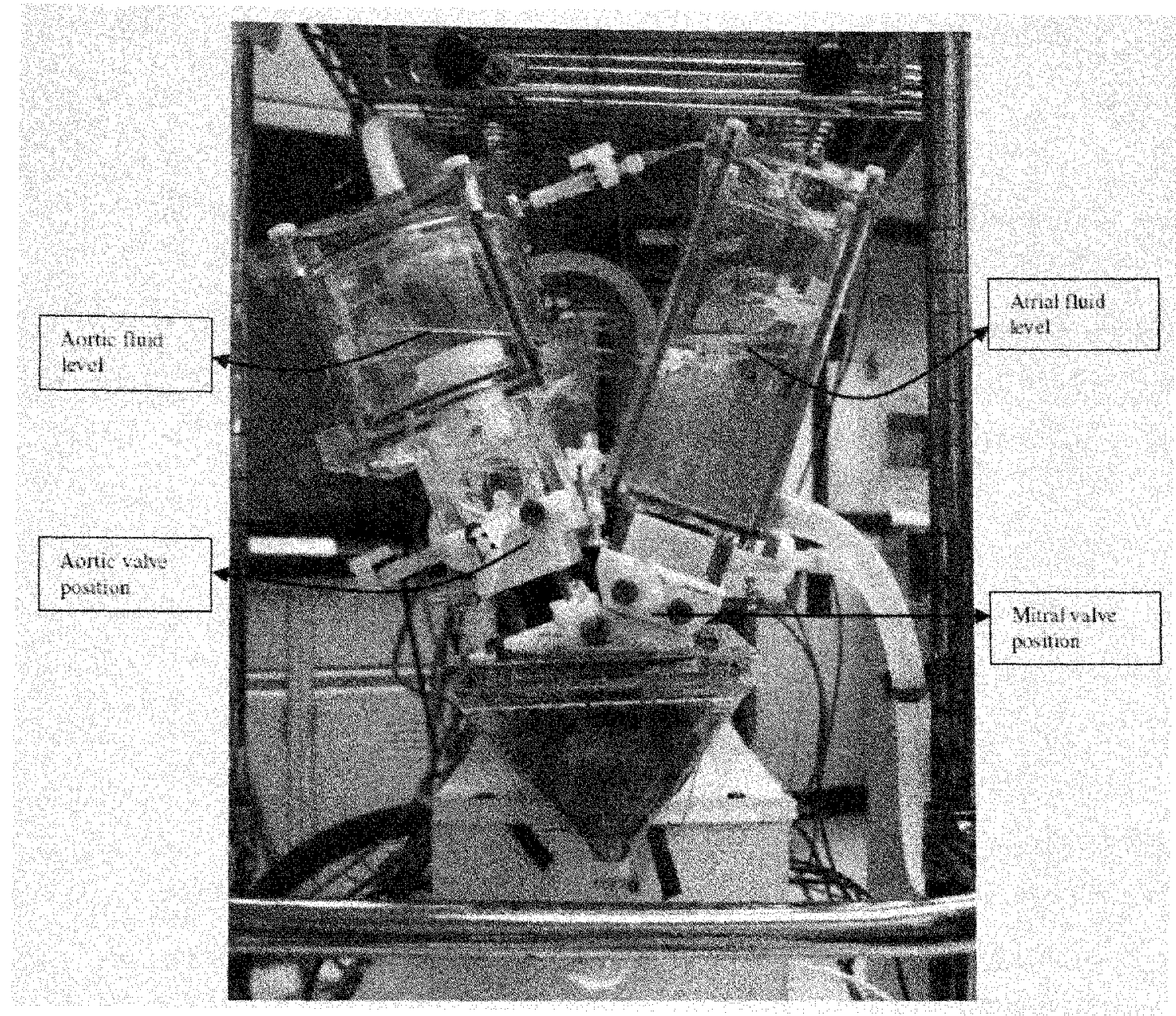

Figure 1: Vivitro Systems, Ine Superdup'r Left Heart Simulator.

FIU - CVEC Vivitro Superdup'r protocol by Siobhain Gallocher

Procedure

1. Title:Protocol for the Hydrodynamic testing of valves in the Vivitro Superdup'r

SD6891: Model Left Heart and Load (figure1).

2. Objectives:

2.1. To ensure a reliable and repeatable method to test valve hydrodynamics in the Vivitro Superdup'r.

2.2. In so doing, the hydrodynamic results from valves tested on different days and by different individuals can be compared.

3. References:
3.1 .
ANSI/AAMI/ISO 5840: 1996 
3.2. FDA CDRH, 1994

4. Procedure:

SPS3891 Operating Manual

4.1. Blood Analog Solution Preparation

4.1.1. Prepare 2L of the blood analogue solution by combining the

following: $720 \mathrm{~mL}$ Glycerine $+1280 \mathrm{~mL}$ DI $\mathrm{H}_{2} \mathrm{O}+18 \mathrm{~g} \mathrm{NaCl}$

4.1.2. Mix until solution is homogeneous ( $-15 \mathrm{mins})$

4.2. Equipment Set-up

4.2.1. For the Mitral position valve:

4.2.1.1. Place the St. Jude Bileafelt valve in its silicone valve holder and insert it into the mitral position so that the valve opens downwards and fluid flow is from the atrium into the ventricle.

4.2.1.2. Replace and secure the left atrium assembly and associated tubing

4.2.2. For the aortic position valve:

4.2.2.1. Place the valve to be tested in its silicone valve holder, and orient it in position so that fluid flow is upwards, from the ventricle to the aorta.

4.2.2.2. For trileaflet valves, be sure to orient the valve so that it is aligned with the sinus of valsalva (glass sinus).

4.2.2.3. Orient it so that the leaflets are in line with the 3 sinuses and the posts are aligned with the inter-sinus spaces.

4.2.3. Fill the system with the blood analog solution prepared in 4.1

4.2.3.1. The fluid levels are represented in Figure 1 below.

4.2.3.2. Running the system at a low amplitude can accelerate this filling process

4.2.4. Turn on the electromagnetic flow meter and press the alarm button.

4.2.4.1. Do not turn on flowmeter unless flow probe is submersed in fluid.

4.2.4.2. Be sure to allow the flow meter to warm up for 30 mins prior to use

4.2.5. Connect two catheter-tipped piezoelectric pressure transducers to their associated

4.2.5.1. Handle the pressure transducers very delicately because small mechanical injury can result in transducer damage

4.2.5.2. Record in the laboratory notebook the serial numbers and reference numbers of each of the three components (pressure transducer, control unit, and amplifier) and the manner/order in which they are connected.

4.2.5.3. Tape the catheter tips to the acrylic top of the Superdup'r unit for the calibration/zeroing procedure.

4.2.5.4. Check that the amplifier settings are as follows: Gain: 5000; $300 \mathrm{~Hz}$ LP: ON; LP: $5 \mathrm{kHz}$; HP: DC

4.3. Pressure and Flow Transducer Scaling and Zeroing

4.3.1. Open the Acqknowledge 3.7.3 software 
When setting up the channels, assign them as follows by inputting the labels in the editable text portion:

4.3.3.2.

Channel A1: Ventricular Pressure

4.3.3.3.

Channel A2: Aortic Pressure

4.3.3.4.

Channel A3: Aortic Flow

4.3.3.5.

Make a note of which pressure transducer $\&$ control box is attached to which channel

4.3.3.6. This is an arbitrary assignment, but once assigned, they must not be switched.

4.3 .3 .7 4.3.3.7.1.

Scale the pressure and flow transducers as follows:

4.3.3.7.1.1. Select the circular button for the Ventricular Pressure and click the scaling button.

4.3.3.7.1.2.

In the pop-up box that appears, enter 0 and 100 for the two scale values, input $\mathrm{mmHg}$ in the Units box, and select the use mean value in the Options box

4.3.3.7.1.3.

Make sure the pressure transducer control box for the ventricular pressure transducer is set to Standby and hit Call 5 times in succession.

4.3.3.7.1.4.

Change the pressure transducer control box setting to 100 and hit the $\mathrm{Cal} 2$ button 5 times in succession.

4.3.3.7.1.5. Once completed select $\mathrm{OK}$ and set the pressure transducer control boxback to Standby.

4.3.3.7.1.6.

Repeat this procedure for the Aortic

Pressure

4.3.3.7.2.

Flow Transducer

4.3.3.7.2.1.

Select the circular button for the Aortic

Flow and click the scaling button.

$4.3,3.7 .2 .2$.

In the pop-up box that appears enter 0 and 1

for the two Input Volts values, 1 and 100 for the scale values, and enter $\mathrm{L} / \mathrm{min}$ in the Units box

4.3.3.7.2.3.

This is the standard setting when the

flowmeter Range is set to Hi-C.

Once completed select $O K$.

4.3.4. Close the Input Channels Box

4.3.5. Transducer Zeroing

4.3.5.1. For the five data analysis pull-down menus change the first 3 to measure mean Ventricular Pressure ( $\mathrm{CH} 1)$, Aortic Pressure $(\mathrm{CH} 2)$, and Aortic flow (CH3).

4.3.5.2.

Pressure Transducer

4.3.5.2.1. Change the pressure transducer control box settings for both the ventricular and aortic pressure to transducer. 
4.3.5.2.2.

With the transducers exposed to atmosphere, select the start button on the bottom right of the Acqknowledge screen, and adjust the transducer balance until the output is approximately zero $( \pm 0.05)$.

4.3.5.2.3. In order to approximate this more accurately it may be necessary to adjust the vertical scale on the right hand side of the plot.

4.3.5.2.4. Measure approximately 10 secs of data to determine the numerical mean value.

4.3.5.2.5. zeroed.

Lock the balance in place when the pressures are

4.3.5.2.6. box back to Standby.

Once completed set the pressure transducer control

4.3.5.2.7. Whenever pressure transducers are not in use, the

4.3.5.3. pressure transducer control box should be set to Standby

4.3.5.3.1. Select the start button on the bottom right of the screen, and adjust the zero on the Flowmeter until the output is approximately zero $( \pm 0.05)$.

4.3.5.3.2.

Measure approximately 10 secs of data to determine the numerical mean value.

4.4. Flow and Pressure Transducer Calibration

4.4.1. Flow transducer

4.4.1.1. Flow transducer calibration must be performed every 3 months. Refer to the Flow Transducer Calibration Protocol.

\subsubsection{Pressure transducer}

4.4.2.1. Pressure transducer calibration must be performed prior to any experimental measurements.

4.4.2.1.1. Connect the Ventricular pressure transducer to the 3-way stopcock of the pressure calibration set-up (mercury manometer to record pressure \& sphygmomanometer bulb to induce pressure).

4.4.2.1.2.

Change the pressure transducer control box settings to transducer.

4.4.2.1.3. With the sphygmomanometer bulb, exert 100 $\mathrm{mmHg}$ while simultaneously recording the pressure with the Acqknowledge software.

Check the mean pressure recorded with Acqknowledge is $100 \mathrm{mmHg}$.
4.4.2.1.5.
4.4.2.1.6.
Repeat this for an applied pressure of $50 \mathrm{mmHg}$.
If, at any stage, the recorded pressure does not

match the applied pressure, repeat procedure 4.3.C.III.a and then 4.4.B.I.a-f.

4.5. Pressure Transducer Set-up 
4.5.1. Insert catheter-tipped piezoelectric pressure transducers into ventricular and aortic positions.
4.5.1.1.
For the ventricular pressure, insert the pressure transducer through the 3 -way stop-cock as shown.

4.5.1.2. Thread the catheter through the port until it is located in the center of the ventricle.

4.5.1.3. For the aortic pressure, insert the pressure transducer through the 3-way stop-cock.
4.5.1.4. Thread the catheter through the port until it is located in the center of the aorta.
4.5.1.5. Keep the stop-cock open to the transducer as long as it is in position. DO NOT close the stop cock as this will result in damage to the pressure transducer.
4.5.1.6. The stop-cock can be closed when the transducer is removed. Failure to close the stop-cock under these conditions will result in fluid loss from the system.

4.5.2. Take baseline data

4.5.2.1. Once transducers are in place, measure the baseline data:

4.5.2.2. Make sure the Superpump Control Box is off.

4.5.2.3. Using the Acqknowledge software, select the start button on the lower right hand side of the screen and allow it to run to completion ( $30 \mathrm{secs}$ ). Save the file under its valve type/lot\# and entitle it "Baseline" (eg. C:IMy Documents IVLV 60\Baseline)
4.5.2.4.
This data will be used during data analysis.

4.6. Experimental Measurement

4.6.1. The standard experimental cardiac output/heart rate combinations are presented in. Any additional cardiac outputs can be included at the discretion of the evaluator.

4.6.2. System Tuning

4.6.2.1. Change the pressure transducer control box settings for both the ventricular and aortic pressure to transducer.

4.6.2.2. Use the Select Wave Knob on the Waveform Generator to select the cardiac output required: $1: 45 \mathrm{BPM} ; 2: 70 \mathrm{BPM} ; 3: 100$ BPM; 4: 120 BPM

4.6.2.3. Increase the amplitude on the Superpump Control Box until the approximate cardiac output is achieved.

4.6.2.4. This is done iteratively. Dial up the amplitude to approximately 2.5 and adjust the resistance until the pressure gauge reads $120 / 80$.

4.6.2.5. Measure the cardiac output and mean aortic pressure using the Aqcknowledge software

4.6.2.6. Highlight 5 waves from beginning systole to end diastole and measure the mean flow (i.e. cardiac output) and aortic pressure.

4.6.2.7. The mean aortic pressure should be $\sim 95 \mathrm{mmHg}$ 
4.6.2.8. Adjust the amplitude on the Superpump Control Box and the resistance, and repeat 4.6.B.III.b until the desired flow is achieved.

4.6.2.9. Check that the Silicone Ventricle Membrane is neither over-inflating or -contracting

4.6.2.10. If it is doing either, insert or remove air from the right port on the Viscoelastic Impedance Adapter.

4.7. Once mean cardiac output and aortic pressure are set and the ventricle is functioning correctly, video the valve function and record a full range of data (30secs) by selecting the Start button. Save the data in the same folder as the baseline data and include the cardiac output and heart rate in the filename.

4.7.1. Digital video recording can interfere with the flow transducer, so take a segment of video prior to recording any data. Switch the video recorder off and remove it from the area for data acquisition.

4.7.2. For example, if a valve with Lot\# TRV-VLV-0004-R060 is tested at $70 \mathrm{BPMs}$ and $6 \mathrm{~L} / \mathrm{min}$, it can be saved as:

4.7.3. C: C:My Documents IVLV 60170-6

4.7.4. Repeat 4.6.B for each of the cardiac output/heart rate combinations.

\begin{tabular}{|c|c|c|c|c|}
\hline Heart Rate & 45 & 70 & 100 & 120 \\
\hline \multirow{3}{*}{ Co } & 2.3 & 3.9 & 6.0 & 7.8 \\
\cline { 2 - 5 } & 3.6 & 5.6 & 8.0 & 9.6 \\
\cline { 2 - 5 } & 5.0 & 7.4 & 10.0 & 11.4 \\
\hline
\end{tabular}

SOP 05

\section{TC PHV 4 SERIES FABRICATION PROCESS}

1. Title: TC PHV fabrication procedure.

2. Author: Thomas E. Claiborne, III

3. Scope: This document is intended to describe the process of manufacturing a TC PHV 4 series prototype.

4. References: Deurig et al. 2000, Gallocher et al. 2006, www.nitinol.com

5. Materials:

5.1. 5.1. Bard OEM Components, non-sterile, P04081 polyester fabric; style: 6102

5.2. Small Parts, Inc. super-elastic nitinol wire, 36 inch length, 0.022 inch OD, 0.012 inch OD 2 each

5.3. Small Parts, Inc. XTW 304 SS Hypo-Tube 6 inch lengths, ID 0.025 inch, ID 0.050 inch 1 each.

5.4. SIBS pellets

5.5. Toluene solvent

5.6. 4-0 Ethibond Excel braided polyester suture, 36 inch length, RB-1 taper needle, 4 each 
5.7. Elmer's Krazy Glue, 1 tube

5.8. Small Parts, Inc. 302 SS ball bearings, $3 / 8$ inch, 3 each

5.9. Aluminum foil

5.10. Invisible tape

5.11. Water

6. Equipment:

6.1. Gallocher drying plate

6.2. Free standing grip

6.3. Needle driver

6.4. Suture scissors

6.5. Forceps

6.6. Scalpel

6.7. Fume Hood

6.8. Glass Bowl

6.9. Bubble level

6.10. Furnace capable of reaching $500^{\circ} \mathrm{C}$

6.11. Claiborne nitinol annealing fixture

6.12. C-clamps, 5 each

6.13. Hexagonal wrenches

6.14. Nut driver

6.15. Furnace tongs

6.16. Heat resistant gloves

6.17. 5-gallon bucket

6.18. Fabric cutter

6.19. Small flat-head screw driver

7. Procedure:

7.1. Shaping the Nitinol Wire

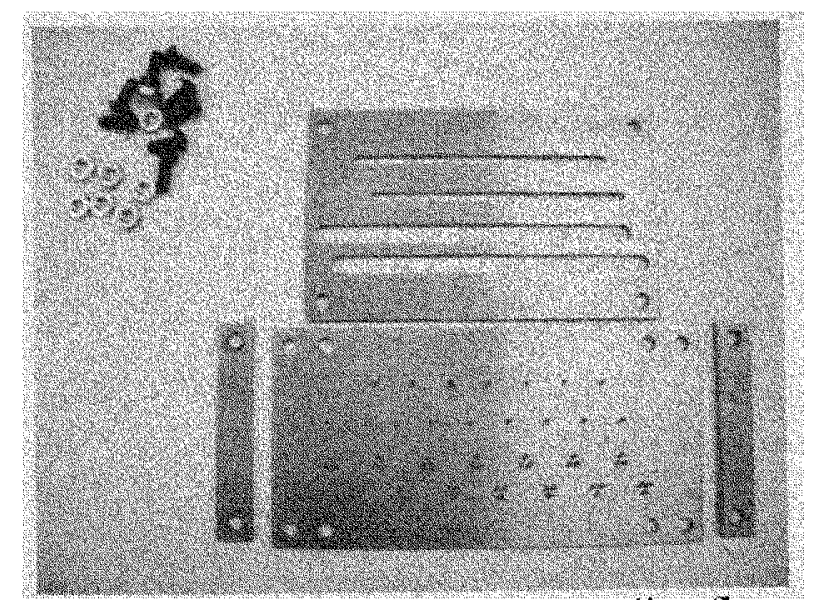

Figure 1: Claiborne nitinol wire annealing fixture.

7.1.1. Take the base plate of the Claiborne annealing fixture (the one with the pins) and clamp it to the table top using a C-clamp. 
7.1.2. Using another C-clamp, secure the end of the 0.022 OD nitinol wire near the edge of the base plate.

7.1.3. Begin threading the wire around the pins in the plate that are patterned to make tear drop shaped bends in the wire (clusters of three pins in a triangle).

7.1.4. Keep the wire taught and guide it into the pin slots using a flathead screw driver. Failure to do so will allow the wire to pop out of the fixture.

7.1.5. Once the entire pin pattern has been threaded, secure the end of the wire with a third C-clamp.

7.1.6. Now repeat the procedure using two 0.012 OD nitinol wires and the single pin pattern that will create a sinusoidal shape.

7.1.7. Lay each wire on top of the other.

7.1.8. Place the top plate of the Claiborne annealing fixture onto the bottom plate, fitting the slots over the pins.

7.1.9. Place the two end clamps on the bottom plate.

7.1.10. Secure all plate with the screws and nuts (figure 2).

7.1.11. Ensure that they are as tight as possible.

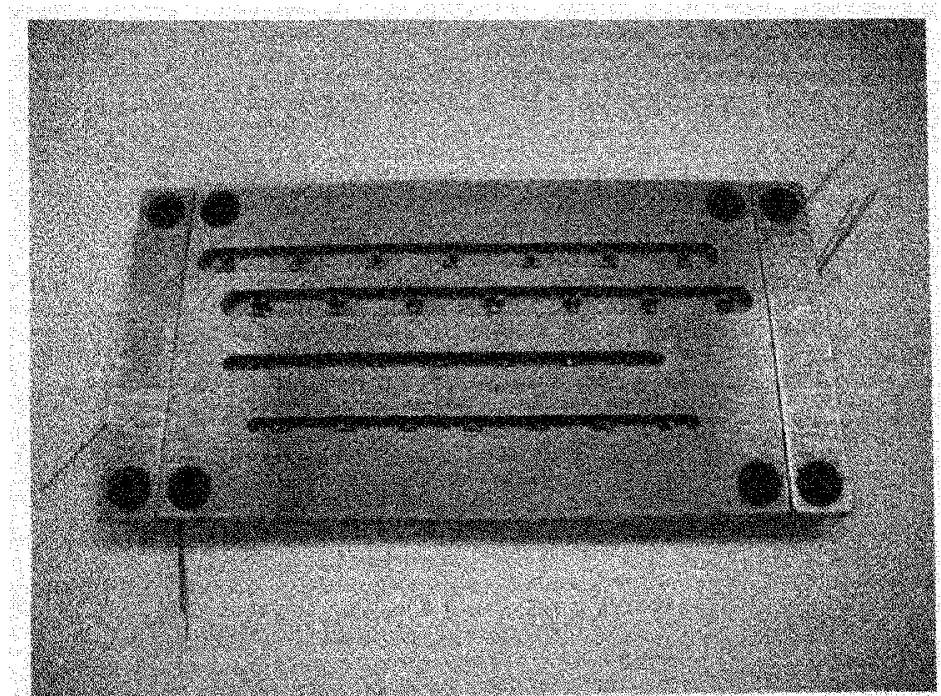

Figure 2: Claiborne annealing fixture assembled with wires in place.

7.1.12. Place the fixture in a cold furnace and set the temperature to $500^{\circ} \mathrm{C}$.

7.1.13. Once the furnace reaches $500^{\circ} \mathrm{C}$ let the fixture soak in the furnace for 5 minutes. Temperature fluctuation during the soak of $+1-20^{\circ} \mathrm{C}$ are acceptable.

7.1.14. During the soak fill a 5-gallon bucket with water $2 / 3$ full.

7.1.15. After 5 minutes turn off the furnace, and using the tongs and heat gloves, carefully remove the fixture and slowly quench it in the bucket of water.

7.1.16. Once it is cool to the touch, dry the fixture and remove the wires. 
7.2.1. Perform this procedure inside a fume hood.

7.2.2. Mix the SIBS pellets and Toluene $15 \%$ by mass such that you have about $50 \mathrm{ml}$ total in a glass jar with a lid then place on a stirring machine.

7.2.3. While the SIBS-toluene mixture is mixing prepare the drying plate.

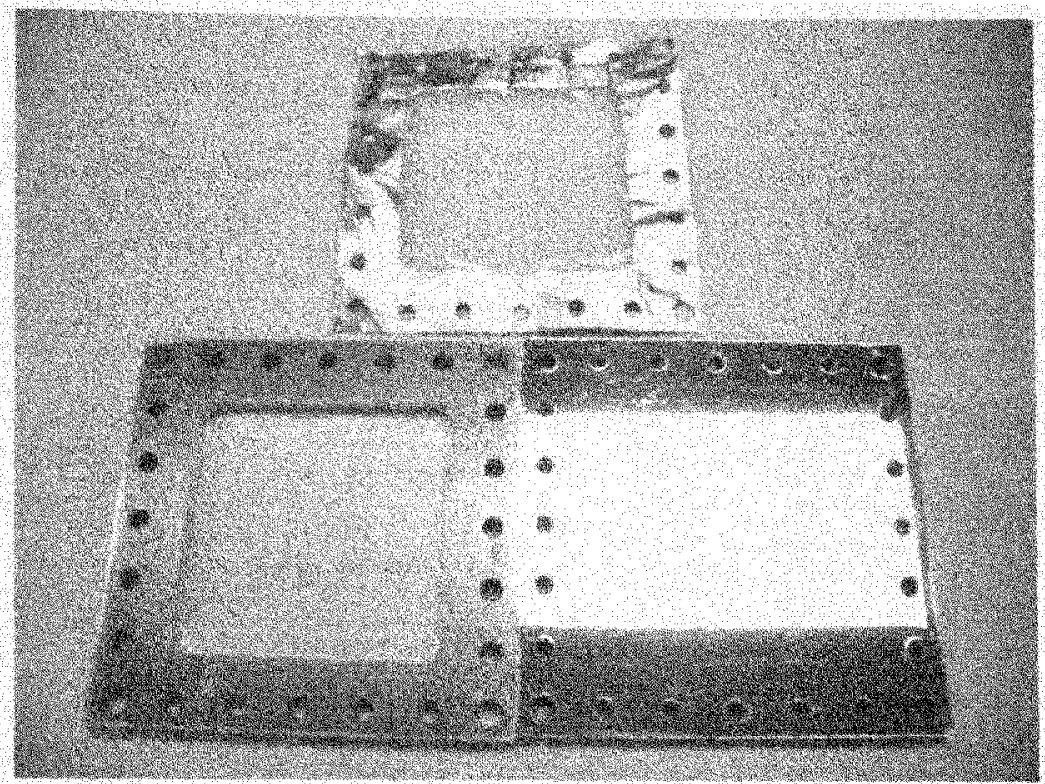

Figure 3: Gallocher drying plate and finished SIBS-polyester sheet shown.

7.2.4. Ensure that the drying plate is clean and dry.

7.2.5. Cut a piece of aluminum foil in the shape of the outer dimension of the drying plate, then from that square, cut out a hole in the shape of the square hole in the drying plate bracket (figure 3 ).

7.2.6. Using the thin transparent tape, secure the aluminum foil gasket to the mirror finish side of the drying plate. Only tape the outer edges. Do not tape the central edge.

7.2.7. Cut a $3 \times 3$ inch square of the Bard polyester fabric.

7.2.8. Using the tape secure the fabric to the mirror finish side of the drying plate on top of the aluminum foil gasket.

7.2.9. While taping down the fabric stretch it taught, but do not cover the screw holes with fabric.

7.2.10. Using a scalpel, incise the tape/foil over each screw hole.

7.2.11. Place the drying plate bracket on top of the fabric and secure it using the screws.

7.2.12. Using the 4 long corner screws and the bubble level, level the plate in the fume hood.

7.2.13. Using a syringe, draw up 7-8 cc of SIBS-toluene mixture. (200-250 microns thickness will result) 
7.2.14. Carefully pour the mixture onto the fabric, ensuring coverage of the entire surface. If air bubbles are present place the fixture into a vacuum chamber until it is debubbled.

7.2.15. Place a glass bowl over the plate and let it cure for 24 hours.

7.2.16. After 24 hours prop-up an edge of the glass bowl for ventilation purposes and allow another 24 hours of curing time.

7.2.17. Then remove the fabric from the plate.

7.3. Stent Formation

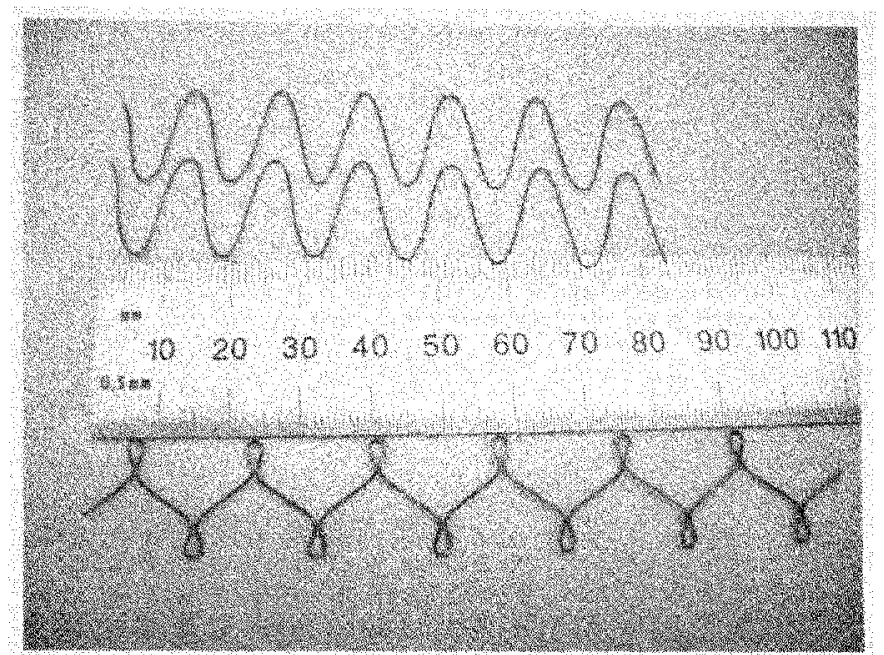

Figure 4: Nitinol wire shown cut to length for stent formation.

7.3.1. Cut each wire so that there are only 6 bends on each side (figure 4).

7.3.2. Join the ends of the wire with about a $1 \mathrm{~cm}$ length of hypo-tubing and crimp the tubing.

7.3.3. Place the stents into the ends of an unused silicone aorta or another $19 \mathrm{~mm}$ ID tube.

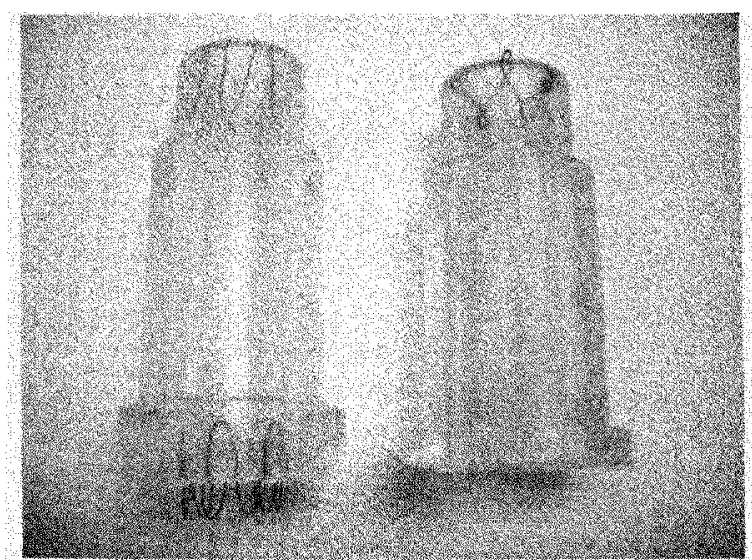

Figure 5: Stents shown inside old SAs for gluing and drying. 
7.3.4.

Then add the glue to the hypo-tubing. Capillary action will draw the glue into the tubing.

7.3.5. Once glue has dried, about 30 minutes, remove the stents and scrape off the excess glue using a scalpel.

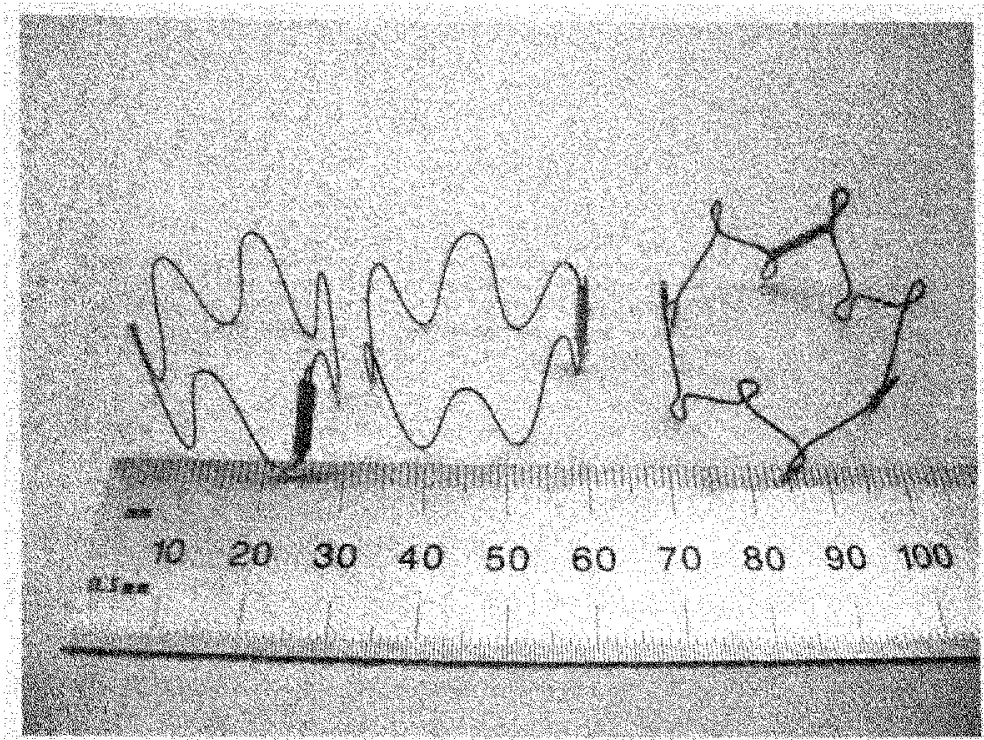

Figure 6: Assembled stents.

7.4. Valve Assembly

7.4.1. Cut a $20 \mathrm{~cm} \times 65 \mathrm{~cm}$ rectangle from the SIBS-polyester sheet.

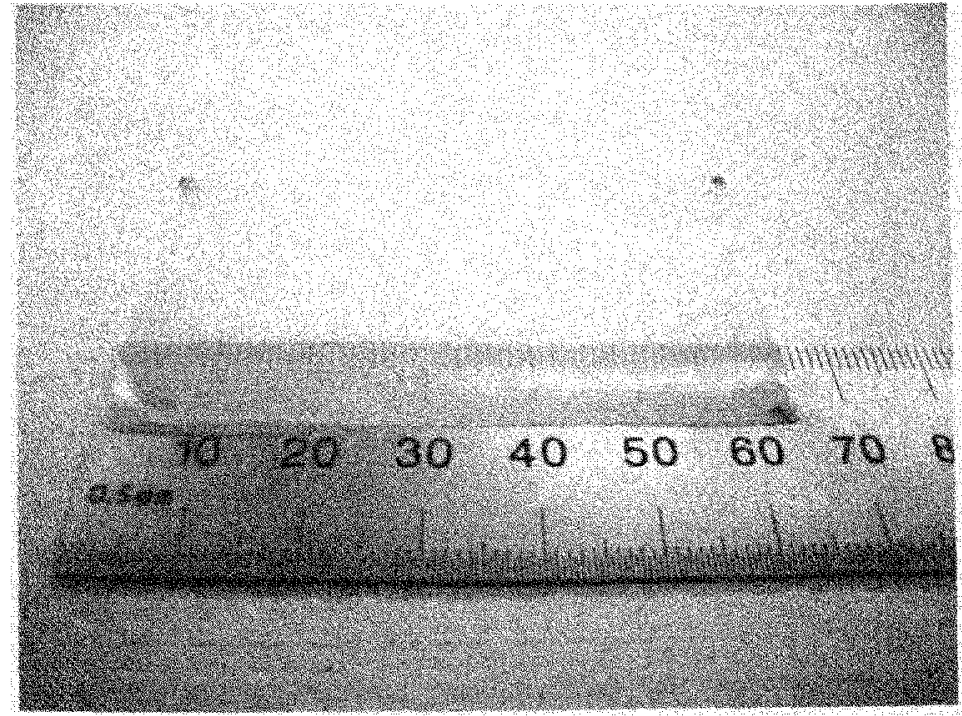

Figure 7: Valve material cut to size.

7.4.2. Place the sheet into the grip fixture and form the sheet into a cylinder ensuring that the smooth shiny side is the inside surface (figure 8). 


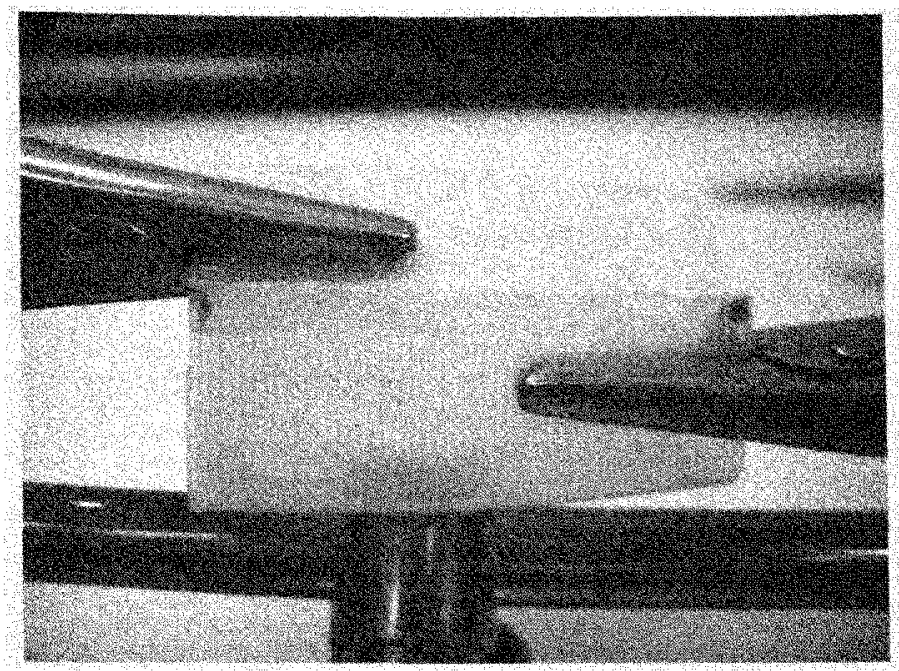

Figure 8: Valve material prepared for suturing.

7.4.3. Using an in-out purse-string style stitch secure the two overlapping ends of the sheet. They should over lap by about 2-3 mm (figure 9).

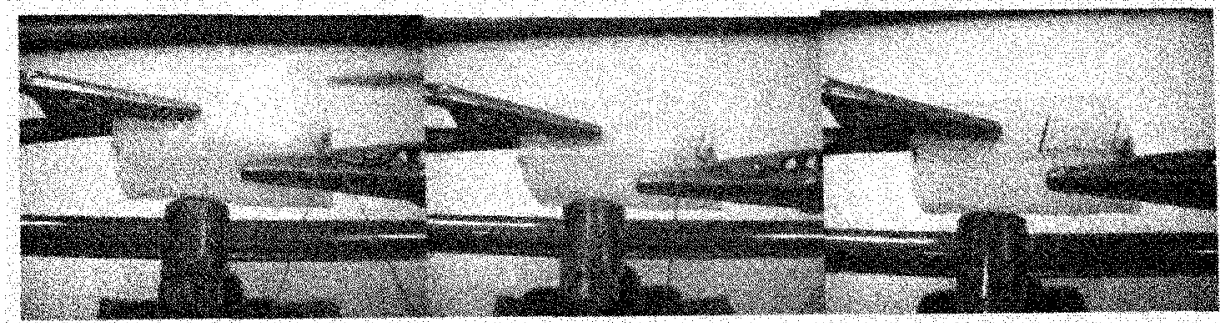

Figure 9: Stitching technique shown.

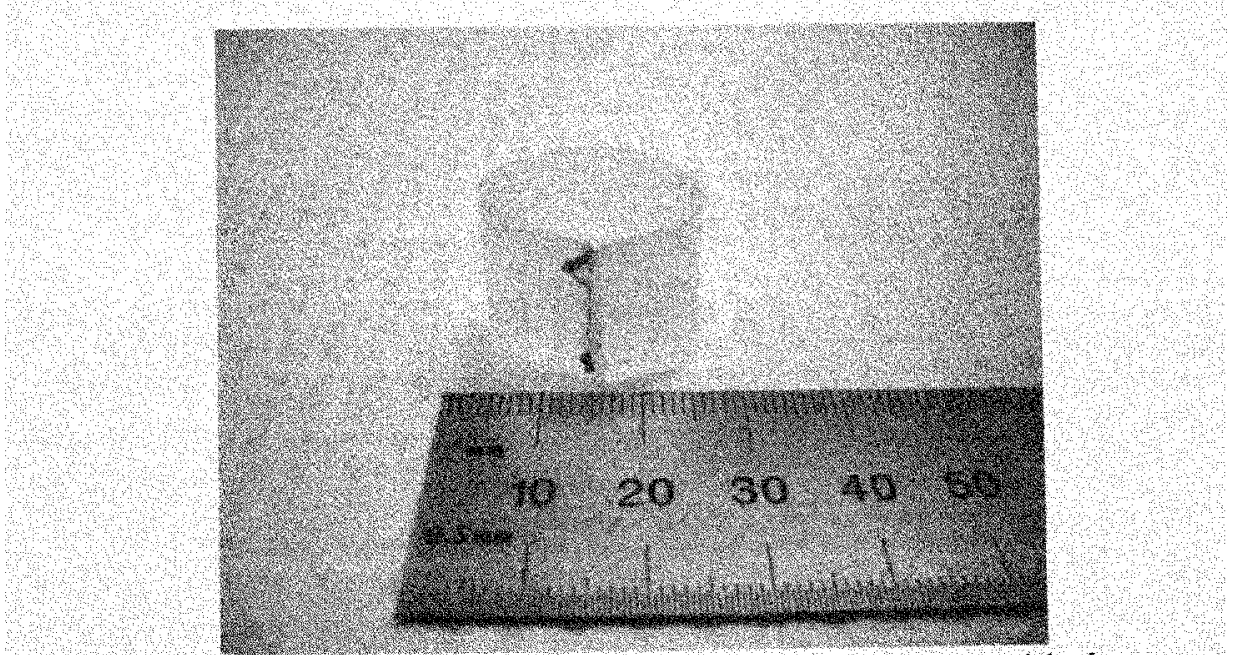

Figure 10: Final shape of valve material prior to marriage with the stents.

7.4.4. Fold the valve material cylinder such that a $5 \mathrm{~mm}$ high cuff is made on the outside of the valve.

7.4.5. Place the two sinusoidal stents into the cuff (figure 11). 


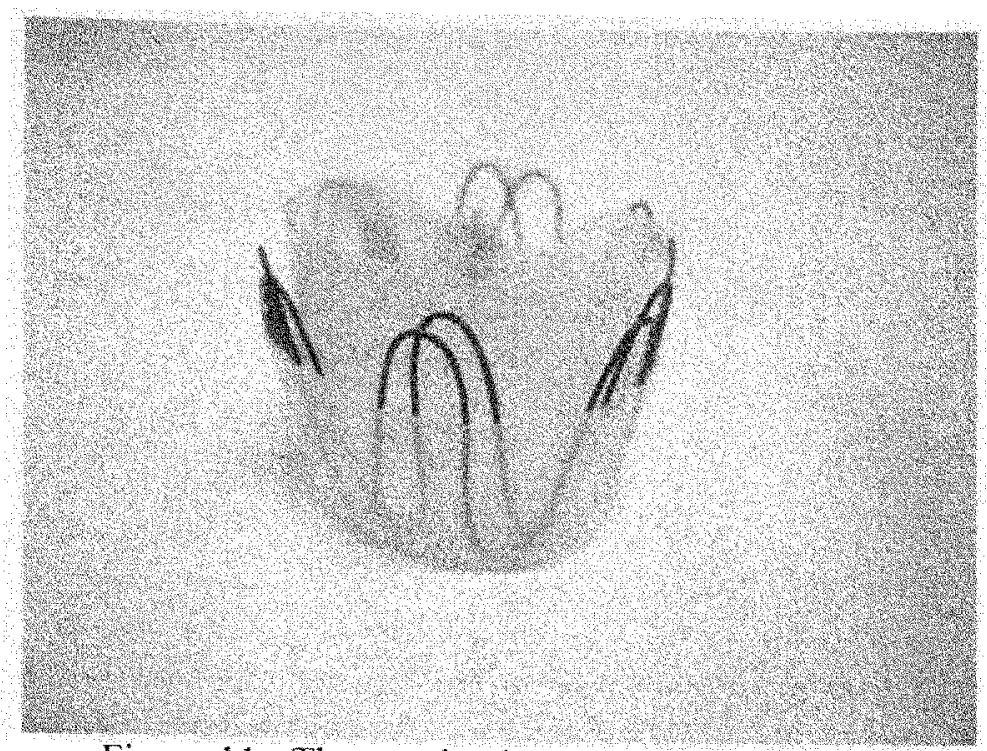

Figure 11: The proximal stents in the valve cuff.

7.4.6. Arrange the stents so that they are $180^{\circ}$ out of phase.

7.4.7. Suture the crossing sections of the stents to the cuff using single stitches(figure 12).

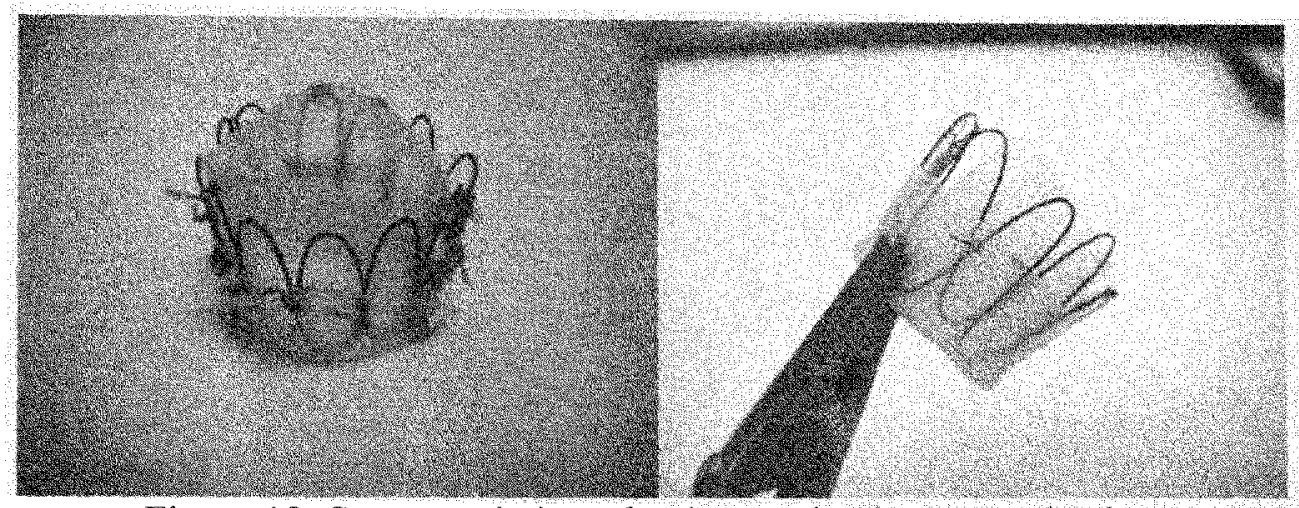

Figure 12: Suture technique for the proximal stents and valve.

7.4.8. Then suture the distal edge of the valve to each of three of the bends of one of the stents at equal distances, i.e. out of 6 bends, every other bend will be secured to the distal portion of the valve material. This will form the trileaflet valve (figure 12).

7.4.9. Now secure the distal fixation stent to the distal edge of the proximal stent-valve assembly looping the sutures around twice between each of 6 bends in the distal and primal stents. Tie at least 6 knots. 


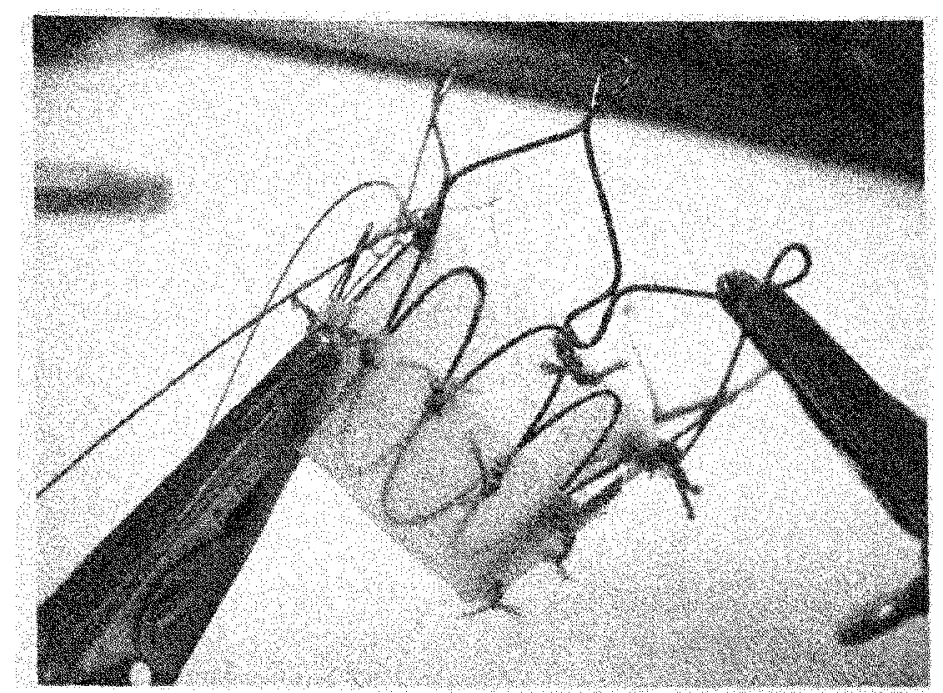

Figure 13: Adding the fixation stent to the PHV.

7.5. Annealing the PHV

7.5.1. Place the finished PHV onto the $19 \mathrm{~mm}$ OD SA mold insert via the base of the PHV (figure 14).

7.5.2. Wedge one SS ball bearing behind each leaflet, 3 each.

7.5.3. If needed place a large BB on top.

7.5.4. Place the assembly in an oven set to $60^{\circ} \mathrm{C}$ for two hours.

7.5.5. Remove and allow to cool before testing.

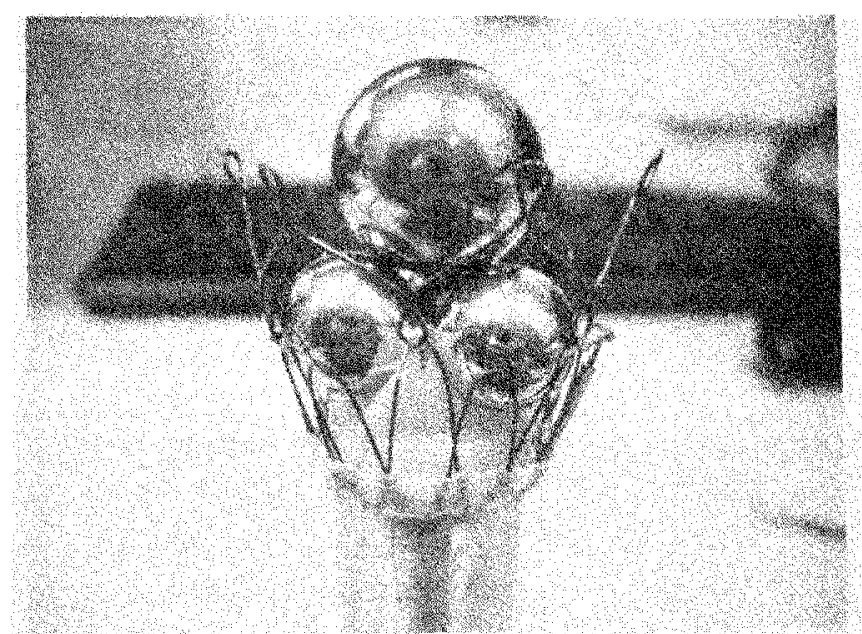

Figure 14: Shape setting the leaflets to hemispherical shapes. 


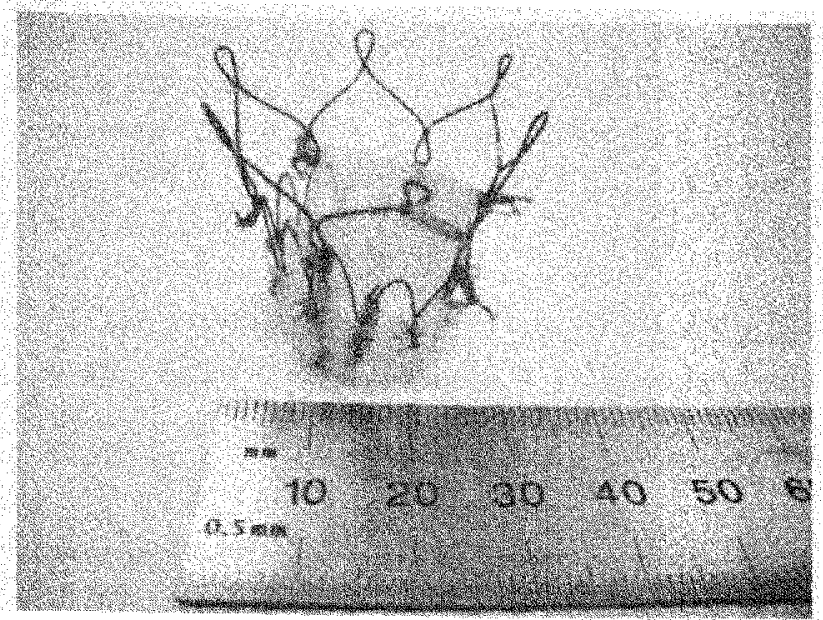

Figure 15: Final TC PHV 4 series design.

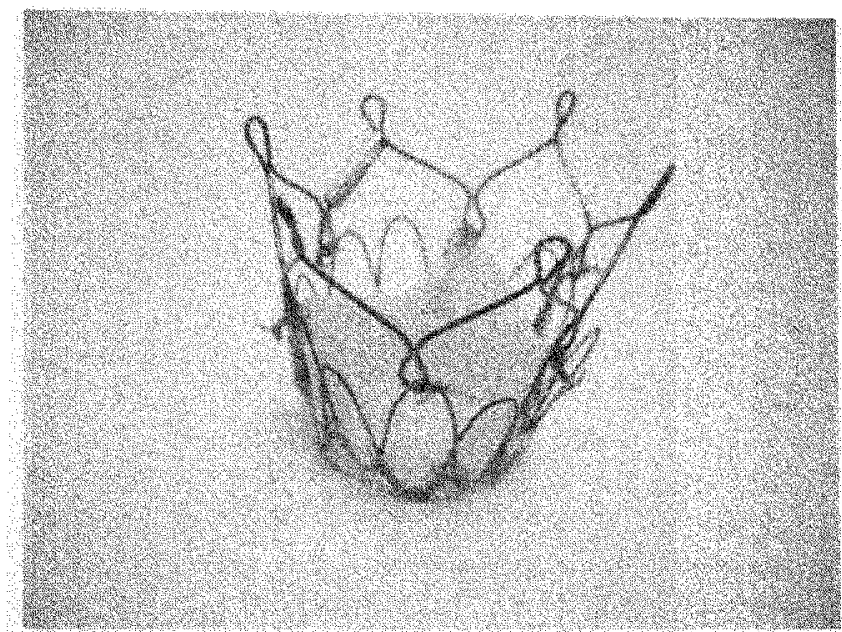

Figure 16: Alternative suture technique shown on the outer cuff. A running stitch all the way around the bottom half of each stent bend.

\section{USPTO SEARCH RESULTS}

a. 6168614- Andersen et al. "Valve prosthesis for implantation in the body" 2001

b. 5411552- Andersen et al. "Valve prosthesis for implantation in the body and a catheter for implanting such valve prosthesis" 1995

c. 5840081- Andersen et al. "System and method for implanting cardiac valves" 1998

d. 5855601- Bessler et al. "Artificial heart valve and method and device for implanting the same" 1999

e. 4056854- Boretos et al. "Aortic heart valve catheter" 1977

f. 4777951-Cribier et al. "Procedure and catheter instrument for treating patients for aortic stenosis" 1988

g. 7175656- Khairkhahan "Percutaneous transcatheter heart valve replacement" 2007 
h. 5957949- Leonhardt et al. "Percutaneous placement valve stent" 1999

i. 3671979- Moulopoulos "Catheter mounted artificial heart valve for implanting in close proximity to a defective natural heart valve" 1972

j. 5397351- Pavcnik et al. "Prosthetic valve for percutaneous insertion" 1995

k. 7041132- Quijano et al. "Percutaneously delivered heart valve and delivery means thereof" 2006

1. 6454799-Schreck "Minimally-invasive heart valves and methods of use" 2002

m. 6767362-Schreck "Minimally-invasive heart valves and methods of use" 2004

n. 6974476- McGurkin, Jr. et al. "Percutaneous aortic valve" 2005

o. 6482228- Norred "Percutaneous aortic valve replacement" 2002

p. 7018408- Bailey et al. "Endoluminal cardiac and venous valve prostheses and methods of manufacture and delivery thereof" 2006

q. 6830584- Seguin "Device for replacing a cardiac valve by percutaneous route" 2004

\section{WIPO SEARCH RESULTS}

i. (WO/2000/041652) PROSTHETIC HEART VALVE IMPLANTABLE BY CATHETER INSERTION OR SURGICALLY

ii. (WO/1991/017720) A VALVE PROSTHESIS FOR IMPLANTATION IN THE BODY AND A CATHETER FOR IMPLANTATING SUCH VALVE PROSTHESIS

iii. (WO/2007/013999) COLLAPSIBLE HEART VALVE WITH POLYMER LEAFLETS

iv. (WO/2005/087140) PERCUTANEOUS HEART VALVE PROSTHESIS

v. (WO/2005/009285) PERCUTANEOUS HEART VALVE

vi. (WO/2004/093728) PERCUTANEOUS TRANSCATHETER HEART VALVE REPLACEMENT 